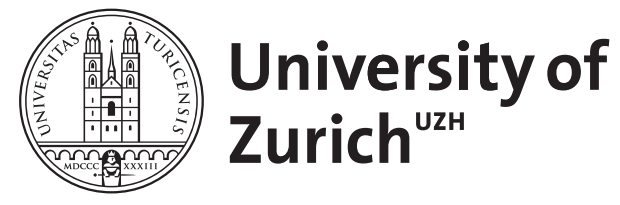

Zurich Open Repository and Archive

University of Zurich

University Library

Strickhofstrasse 39

CH-8057 Zurich

www.zora.uzh.ch

Year: 2015

Fatigue: Neurobiologische, klinische und therapeutische Aspekte eines häufigen Symptoms

Valko, P O

Posted at the Zurich Open Repository and Archive, University of Zurich

ZORA URL: https://doi.org/10.5167/uzh-137294

Habilitation

Originally published at:

Valko, P O. Fatigue: Neurobiologische, klinische und therapeutische Aspekte eines häufigen Symptoms. 2015, University of Zurich, Faculty of Medicine. 
Klinik für Neurologie

UniversitätsSpital Zürich

Prof. Dr. med. M. Weller

\section{Arousal disturbances in neurological disorders}

\section{HABILITATIONSSCHRIFT}

zur Erlangung der Venia legendi der Universität Zürich

Dr. med. Philipp Oliver Valko

Zürich, 2014 
Klinik für Neurologie

UniversitätsSpital Zürich

Prof. Dr. med. M. Weller

\section{Arousal disturbances in neurological disorders}

\section{ZUSAMMENFASSUNG DER HABILITATIONSSCHRIFT}

zur Erlangung der Venia legendi der Universität Zürich

Dr. med. Philipp Oliver Valko

Zürich, 2014 


\section{Included Papers}

1. Valko PO, Bassetti $C L$, Bloch $K E$, Held U, Baumann $C R$. Validation of the fatigue severity scale in a Swiss cohort. Sleep 2008; 31:1601-1607.

2. Valko PO, Waldvogel D, Weller M, Bassetti CL, Held U, Baumann CR. Fatigue and excessive daytime sleepiness in idiopathic Parkinson's disease differently correlate with motor symptoms, depression and dopaminergic treatment. Eur J Neurol 2010; 17:14281436.

3. Sommerauer M, Valko PO, Werth E, Baumann CR. Excessive sleep need following traumatic brain injury: a case-control study of 36 patients. J Sleep Res 2013; 22:634-639.

4. Marti $I^{\star}$, Valko PO*, Khatami R, Bassetti CL, Baumann CR. Multiple sleep latency measures in narcolepsy and behaviourally induced insufficient sleep syndrome. Sleep Med 2009; 10:1146-50.

( ${ }^{*}$ equal contribution)

5. Baumann CR, Bassetti CL, Valko PO, Haybaeck J, Keller M, Clark E, Stocker R, Tolnay $\mathrm{M}$, Scammell TE. Loss of hypocretin (orexin) neurons with traumatic brain injury. Ann Neurol 2009; 66:555-559.

6. Valko PO, Gavrilov YV, Yamamoto M, Reddy H, Haybaeck J, Mignot E, Baumann CR, Scammell TE. Increase of histaminergic tuberomammillary neurons in narcolepsy. Ann Neurol 2013; 74:794-804.

7. Valko PO, Khatami R, Baumann CR, Bassetti CL. No persistent effect of intravenous immunoglobulins in patients with narcolepsy with cataplexy. J Neurol 2008; 255:19001903.

8. Poryazova R, Tartarotti S, Khatami R, Baumann CR, Valko P, Kallweit U, Werth E, Bassetti CL. Sodium oxybate in narcolepsy with cataplexy: Zurich sleep center experience. Eur Neurol 2011; 65:175-182.

9. Kaiser PR*, Valko PO*, Werth E, Thomann J, Meier J, Stocker R, Bassetti CL, Baumann CR. Modafinil ameliorates excessive daytime sleepiness after traumatic brain injury. Neurology 2010; 75:1780-1785.

( * equal contribution)

10. Kallweit U, Baumann CR, Harzheim M, Hidalgo H, Pöhlau D, Bassetti CL, Linnebank M, Valko PO. Fatigue and sleep-disordered breathing in multiple sclerosis: A clinically relevant association? Mult Scler Int 2013:286581. 


\section{Summary}

Arousal disturbances are common and disabling complaints among patients with various neurological disorders. The term arousal disturbances refers to a group of symptoms with quantitative reduction or qualitative alteration of normal wakefulness, encompassing, among others, hypersomnia, excessive daytime sleepiness and fatigue. Hypersomnia means increased sleep need per 24 hours, often but not necessarily accompanied by daytime sleepiness. Excessive daytime sleepiness despite adequate nighttime sleep implies increased sleep pressure due to impairment of the normal arousal system in the brain. Fatigue, eventually, represents an ill-defined, subjective experience characterized by a loss of energy, rapid exhaustion and physical and/or mental tiredness. The collection of papers included in this habilitation treatise (I) illustrates the challenges in the clinical and diagnostic assessment of arousal disturbances, (II) discusses recent neuropathological contributions, and (III) presents different therapeutic approaches.

\section{Diagnostic approach to a frequent and disabling clinical problem}

Despite the increased awareness of the high prevalence and clinical relevance of arousal disturbances in neurological disorders, clinicians still struggle with many diagnostic difficulties. First, objective measurement of arousal disturbances is often challenging. This is particularly true in the case of fatigue, which is a subjective experience that currently can be assessed only in a semi-quantitative manner by questionnaires. Second, different arousal disturbances may coexist in the same patient, making it difficult for the treating physicians to differentiate them. Third, clinicians need a high degree of suspicion to diagnose arousal disturbances, because patients may not be aware of and thus not report them. Indeed, the regular use of objective tools such as the mean sleep latency test (MSLT) or actigraphy have revealed a considerable discrepancy between subjective and objective measures of arousal disturbances. Finally, different neurological disorders may produce similar results in sleep lab examinations (e.g. similar mean sleep latency in the MSLT), making the definition of additional features for differentiation necessary.

In the first part, we present four papers exemplifying the above-mentioned diagnostic difficulties. In the first paper, we translated and validated the Fatigue Severity Scale (FSS) in a large sample $(n=1306)$ and for different neurological disorders, thereby providing a reliable, valid and very useful tool for detecting and monitoring fatigue, both for clinical and research purposes. ${ }^{1}$ In the second paper, we demonstrated in patients with idiopathic Parkinson's disease that fatigue and excessive daytime sleepiness are often overlapping yet in many aspects distinct arousal disturbances, as suggested by disparities of their correlation to severity of motor symptoms, disease duration, depression, and dopaminergic treatment. ${ }^{2}$ In the third paper, we assessed sleep need following traumatic brain injury (TBI) and compared subjective estimates with actigraphic measures, thereby identifying a significant underestimation of effective sleep need by TBI patients. ${ }^{3}$ In addition, we found that the combination of hypersomnia and underestimation of posttraumatic sleep-wake disturbances may increase the risk of secondary sleep deprivation with excessive daytime sleepiness. Eventually, in the fourth paper, we compared two patient groups with EDS - patients with narcolepsy with cataplexy and patients with behaviourally induced insufficient sleep syndrome (BIISS) - and showed that analyzing the specific sequence of sleep stages in the MSLT may help differentiating these two conditions. ${ }^{4}$ Specifically, we found that the sleep 
stage sequence non rapid eye movement sleep (NREM) 1 - REM - NREM2 may serve as additional diagnostic parameter for narcolepsy, while the sequence NREM1 - NREM2 REM was more common in BIISS patients.

\section{A neuropathological perspective}

Narcolepsy is a neurological disorder characterized by severe sleepiness, cataplexy, sleep paralysis, hypnagogic hallucinations and fragmented night-time sleep. The study of narcolepsy has proved crucial for our understanding of arousal disturbances in other neurological disturbances and continues to promote substantial advances in the field. Narcolepsy is caused by an extensive loss of the hypothalamic neurons producing the hypocretin (also called orexin) neuropeptides. Subsequently, several groups examined the integrity of these neurons in different neurological disorders commonly associated with chronic daytime sleepiness, and found partial hypocretin cell loss in postmortem brains of patients with idiopathic Parkinson's disease, Alzheimer's disease and Huntington's disease. Arousal disturbances are also very common among TBI survivors, but whether head trauma causes hypocretin cell loss or not was uncertain at that moment. On the other hand, we do not really know whether extensive hypocretin deficiency is solely responsible for all symptoms in narcolepsy with cataplexy.

Thus, in the first paper of the second part of this habilitation treatise we examined brains of deceased TBI patients and compared the number of various hypothalamic neurons, including cells producing hypocretin and melanin-concentrating hormone $(\mathrm{MCH})$, between TBI patients and non-neurological control brains. Our pilot study indicated a significant injury to the hypothalamus, as indicated by reactive gliosis throughout the hypothalamus and loss of several neuronal systems, including cells producing hypocretin and $\mathrm{MCH}^{5}$ Traumainduced decrease of hypocretin signaling may thus contribute to the frequently observed posttraumatic arousal disturbances. In the second paper, we investigated a potential involvement of the histamine system in the neurobiology of narcolepsy. ${ }^{6}$ Histamine neurons play an important role in promoting and maintaining wakefulness, are located in the tuberomammillary nucleus in the posterior hypothalamus, and receive intense excitatory input from hypocretin neurons. Comparing 7 narcolepsy and 12 control brains, we found a striking $94 \%$ increase of histamine neurons in narcolepsy patients, and the gain in histamine neurons was higher in those narcolepsy patients who had the greatest loss of hypocretin neurons. We speculate this increment may reflect an effort to compensate for the reduction of excitatory hypocretin signaling.

\section{Therapeutic challenge}

In the last part of this habilitation treatise, we discuss different treatment strategies for arousal disturbances in neurological disorders. Considering the heterogeneous pathophysiology of arousal disturbances, opportunities of effective therapy may emerge from distinct approaches. Certainly the best way would be to prevent the disease, which is producing the arousal disturbance. We discuss this possibility in the first paper concerning the effect of intravenous immunoglobulins (IVIg) in patients with narcolepsy with cataplexy. ${ }^{7}$ Since current understanding suggests that narcolepsy represents an autoimmune disease, two groups recently treated narcolepsy patients with IVIg and reported a sustained effect on both cataplexy and sleepiness. However, we did not obtain a persistent improvement of narcoleptic symptoms, although temporary (placebo?) effects could be observed. Other 
researchers similarly failed to observe any sustained effect of immunosuppressive treatment in narcolepsy. The next two papers demonstrate that arousal disturbances can either be treated by improving the quality of nocturnal sleep by using hypnotics such as sodium oxybate, ${ }^{8}$ or by reinforcing the activity of certain components of the ascending arousal system, e.g. with stimulants such as modafinil. ${ }^{9}$ Finally, arousal disturbances may emerge not only as a direct consequence of impaired wake-promoting brain circuitries, but also by comorbidities that are associated with the underlying neurological disorder. In this line, in the last paper we discuss the relationship between sleep-disordered breathing and fatigue in patients with multiple sclerosis and whether treatment by continuous positive airway pressure (CPAP) significantly reduces fatigue severity. ${ }^{10}$

\section{Significance of the results}

The importance of arousal disturbances in neurological disorders is represented by the unfavorable discrepancy between the high prevalence and arduous impact on quality of life on one hand, and the currently still marginal insights into the underlying neurobiology and our limited treatment options on the other hand. The first part is dedicated to the clinical and diagnostic assessment of arousal disturbances. The validation of the Fatigue Severity Scale in a large sample size provides a reliable and useful tool for fatigue management, and an ideal addition to the German version of the Epworth Sleepiness Scale, which has also been validated in Zurich (by K. Bloch and colleagues). Additional studies exemplified different and highly characteristic challenges in the diagnosis of arousal disturbances, including overlap of distinct symptoms, discrepancy between subjective and objective measures, and differentiation of neurological disorders producing identical arousal disturbances. The two papers presented in the second part provide a significant contribution to the neurobiological understanding of arousal disturbances. Massive increase of histamine neurons in narcolepsy suggests the capability of the adult human brain to compensate for loss of a specific neuronal population. Finally, the four papers of the third part indicate multiple approaches to improve arousal disturbances. Our single-center study on modafinil in TBI patients provides Class I evidence that modafinil is significantly superior compared to placebo in treating posttraumatic EDS but not fatigue. Nevertheless, our findings emphasize at the same time the necessity of further basic research to produce new and targeted treatment, which is based on neurobiological evidence. Combining clinical perspective and basic neuroscience, the results of the studies are of considerable relevance for both practicing neurologists and fundamental researchers. 


\section{Publication list of Philipp O. Valko, M.D.}

- Original articles included in the cumulative habilitation treatise

- $\quad$ The three most valuable original articles of the cumulative habilitation treatise

प Three particularly valuable original articles not included in the cumulative habilitation treatise

* Equal contribution

\section{Original articles}

1. ${ }^{\square}$ Valko PO, Gavrilov YV, Yamamoto M, Finn K, Reddy H, Haybaeck J, Weis S, Scammell TE, Baumann CR. Damage to histaminergic tuberomammillary neurons and other hypothalamic neurons with traumatic brain injury. Ann Neurol 2014 Nov 1. doi: 10.1002/ana.24298.

IF 11.910

2. ${ }^{\square}$ Valko PO, Siddique A, Linsenmeier C, Zaugg K, Held U, Hofer S. Prevalence and predictors of fatigue in glioblastoma; a prospective study. Neuro Oncol 2014; pii: nou127. IF 5.286

3. Valko PO, Hauser S, Sommerauer M, Werth E, Baumann CR. Observations on sleep-disordered breathing in idiopathic Parkinson's disease. PLoS One 2014; (9(6):e100828.

IF 3.534

4. Sommerauer M, Valko PO, Werth E, Poryazova R, Hauser S, Baumann CR. Revisiting the impact of REM sleep behavior disorder on progression of Parkinson's disease. Parkinsonism Relat Disord $2014 ; 20: 460-462$.

IF 4.126

5. - Kallweit U, Baumann CR, Harzheim M, Hidalgo H, Pöhlau D, Bassetti CL, Linnebank M, Valko $\underline{P O}$. Fatigue and sleep-disordered breathing in multiple sclerosis: A clinically relevant association? Mult Scler Int 2013; doi: 10.1155/2013/286581

6. Baumann CR, Held U, Valko PO, Wienecke M, Waldvogel D. Side and type of motor symptoms at Parkinson's disease onset are linked to disease progression. Mov Disord 2014; 29:207-213. IF 5.634

7. Sherif $E^{*}$, Valko $\mathrm{PO}^{*}$, Overeem S, Baumann CR. Sleep benefit in Parkinson's disease is associated with short sleep times. Parkinsonism Relat Disord 2014; 20:116-118. IF 4.126

8. .- Valko PO, Gavrilov YV, Yamamoto M, Reddy H, Haybaeck J, Mignot E, Baumann CR, Scammell TE. Increase of histaminergic tuberomammillary neurons in narcolepsy. Ann Neurol 2013; 74:794-804.

IF 11.910

9. - Sommerauer M, Valko PO, Werth E, Baumann CR. Excessive sleep need following traumatic brain injury: a case-control study of 36 patients. J Sleep Res 2013; 22:634-639.

IF 2.949 
10. ${ }^{\square}$ Valko PO, Hauser S, Werth E, Waldvogel D, Baumann CR. Heart rate variability in patients with idiopathic Parkinson's disease with and without obstructive sleep apnea syndrome. Parkinsonism Relat Disord 2012; 18:525-531.

IF 4.126

11. Sarikaya H, Fischer A, Valko PO, Weck A, Braun J, Georgiadis D, Baumgartner RW. CT-based intravenous thrombolysis 3-4.5 hours after acute ischemic stroke in clinical practice. Neurol Res 2011; 33:701-707.

IF 1.449

12. Cevoli S, Pizza F, Grimaldi D, Nicodemo M, Favoni V, Pierangeli G, Valko PO, Baumann CR, Montagna P, Bassetti CL, Cortelli P. Cerebrospinal fluid hypocretin-1 levels during the active period of cluster headache. Cephalalgia 2011; 31:973-976.

IF 4.121

13. - Poryazova R, Tartarotti S, Khatami R, Baumann CR, Valko P, Kallweit U, Werth E, Bassetti CL. Sodium oxybate in narcolepsy with cataplexy: Zurich sleep center experience. Eur Neurol 2011; 65:175-182.

IF 1.362

14. •- Kaiser PR*, Valko PO*, Werth E, Thomann J, Meier J, Stocker R, Bassetti CL, Baumann CR. Modafinil ameliorates excessive daytime sleepiness after traumatic brain injury. Neurology 2010; 75:1780-1785.

IF 8.303

15. - Valko PO, Waldvogel D, Weller M, Bassetti CL, Held U, Baumann CR. Fatigue and excessive daytime sleepiness in idiopathic Parkinson's disease differently correlate with motor symptoms, depression and dopaminergic treatment. Eur J Neurol 2010; 17:1428-1436.

IF 3.852

16. Valko $\mathrm{PO}^{*}$, Hänggi $\mathrm{J}^{\star}$, Meyer $\mathrm{M}$, Jung $\mathrm{HH}$. Evolution of striatal degeneration in McLeod syndrome. Eur J Neurol 2010; 17:612-618.

IF 3.852

17. - Baumann CR, Bassetti CL, Valko PO, Haybaeck J, Keller M, Clark E, Stocker R, Tolnay M, Scammell TE. Loss of hypocretin (orexin) neurons with traumatic brain injury. Ann Neurol 2009; 66:555-559.

IF 11.910

18. Pizza F, Biallas M, Wolf $M$, Valko PO, Bassetti CL. Periodic leg movements during sleep and cerebral hemodynamic changes detected by NIRS. Clin Neurophysiol 2009; 120:1329-1334. IF 2.979

19. - Marti I*, Valko PO* , Khatami R, Bassetti CL, Baumann CR. Multiple sleep latency measures in narcolepsy and behaviourally induced insufficient sleep syndrome. Sleep Med 2009; 10:11461150.

IF 3.100

20. Georgiadis D, Arnold M, von Buedingen HC, Valko P, Sarikaya H, Rousson V, Mattle HP, Bousser MG, Baumgartner RW. Aspirin vs anticoagulation in carotid artery dissection: a study of 298 patients. Neurology 2009; 72:1810-1815.

IF 8.303 
21. Valko PO, Siccoli MM, Bassetti CL. Unilateral RLS with predominantly ipsilateral PLMS and variable response to dopaminergic drugs: a variant of idiopathic RLS? Eur J Neurol 2009; 16:430432.

IF 3.852

22. Georgiadis D, Wirz F, von Büdingen HC, Valko P, Hund-Georgiadis M, Nedeltchev K, Rousson V, Baumgartner RW. Intravenous thrombolysis in stroke patients with hyperdense middle cerebral artery sign. Eur J Neurol 2009; 16:162-167.

IF 3.852

23. •- Valko PO, Bassetti CL, Bloch KE, Held U, Baumann CR. Validation of the fatigue severity scale in a Swiss cohort. Sleep 2008; 31:1601-1607.

IF 5.062

24. Siccoli MM, Valko PO, Hermann DM, Bassetti CL. Central periodic breathing during sleep in 74 patients with acute ischemic stroke - neurogenic and cardiogenic factors. J Neurol 2008; 255:1687-1692.

IF 3.841

25. - Valko PO, Khatami R, Baumann CR, Bassetti CL. No persistent effect of intravenous immunoglobulins in patients with narcolepsy with cataplexy. J Neurol 2008; 255:1900-1903. IF 3.841

26. Gemsenjäger E, Valko P, Schweizer I. Basedow disease. From subtotal to total thyroidectomy. Praxis (Bern 1994) 2002; 91:206-215.

\section{Case reports}

27. Valko Y, Werth E, Bockisch CJ, Valko PO, Weber KP. Polysomnography reveals nystagmus from benign paroxysmal positional vertigo. Sleep Med 2014; 15:840-842.

IF 3.100

28. Tamagni C, Mondadori CR, Valko PO, Brugger P, Schuknecht B, Linnebank M. Cerebellum and source memory. Eur Neurol 2010; 63:234-236.

IF 1.362

29. Kaffenberger T, Valko PO, von Meyenburg J, Baráth K, Hewer E, Heppner FL, Jagella CE, Horst A, Siccoli MM. A case of late onset leukoencephalopathy with cerebral calcifications and cysts in a 59-year-old woman. Eur J Neurol 2009; 16:278-281.

IF 3.852

30. Valko PO, Siccoli MM, Schiller A, Wieser HG, Jung HH. Non-convulsive status epilepticus causing focal neurological deficits in CADASIL. J Neurol Neurosurg Psychiatry 2007; 78:1287-1289. IF 5.580

31. Valko P, Busolini E, Donati N, Chimchila Chevili S, Rusca T, Bernasconi E. Severe large bowel obstruction secondary to infection with Actinomyces israelii. Scand J Infect Dis 2006; 38:231-234. IF 1.640 


\section{Reviews}

32. Gavrilov YV, Valko PO. Ivan M. Sechenov (1829-1905). J Neurol 2014 Aug [Epub ahead of print]. IF 3.841

33. Valko PO, Baumann CR. Sergej Nikolajevich Davidenkov (1880-1961). J Neurol 2011; 258:338339.

IF 3.841

34. Bassetti CL, Valko PO. History of the Swiss Neurological Society in the context of the national and international development of neurology. Schweiz Arch Neurol Psychiatr 2009;160:52-65.

35. Valko P, Mumenthaler M, Bassetti CL. History of neurological contributions in the Swiss Archives of Neurology and Psychiatry. Schweiz Arch Neurol Psychiatr 2008;159:157-170.

36. Bassetti CL, Valko P. Poststroke hypersomnia. Sleep Med Clin 2006; 1:139-155.

37. Valko P, Bassetti CL. Aleksej Yakovlevich Kozhevnikov (1836-1902). J Neurol 2006; 253:537-538. IF 3.841

38. Bassetti CL, Bischof M, Valko P. Dreaming: a neurological view. Schweiz Arch Neurol Psychiatr 2005; 156:399-414.

\section{Book chapters}

39. Valko PO, Baumann CR. Sleep disorders after traumatic brain injury. In: Kryger M, Roth T, editors: Principles and Practice of Sleep Medicine, Sixth edition. Elsevier Saunders 2014 (in press).

40. Valko PO, Poryazova R. Secondary narcolepsy. In: Baumann CR, Bassetti CL, Scammell TE, editors: Narcolepsy, pathophysiology, diagnosis, and treatment. Springer, New York, Dordrecht, Heidelberg, London, 2011; 321-339.

41. Bassetti CL, Bischof M, Valko P. Dreaming: a neurological view. In: Mauro Mancia, editor: Psychoanalysis and neuroscience. Springer, Milan, Berlin, Heidelberg, New York, 2006; 351-387. 


\section{Contents}

1. Introduction

2. Diagnostic approach to a frequent and disabling clinical problem

2.1. Validation of the Fatigue Severity Scale in a Swiss cohort

2.2. Fatigue and excessive daytime sleepiness in idiopathic Parkinson's disease differently correlate with motor symptoms, depression and dopaminergic treatment

2.3. Excessive sleep need following traumatic brain injury: a case-control study of 36 patients

2.4. Multiple sleep latency measures in narcolepsy and behaviourally induced insufficient sleep syndrome

3. A neuropathological perspective

3.1. Loss of hypocretin (orexin) neurons with traumatic brain injury 21

3.2. Increase of histaminergic tuberomammillary neurons in narcolepsy 23

4. Therapeutic challenge 26

4.1. No persistent effect of intravenous immunoglobulins in patients with 26 narcolepsy with cataplexy

4.2. Sodium oxybate in narcolepsy with cataplexy: Zurich sleep center experience

4.3. Modafinil ameliorates excessive daytime sleepiness after traumatic brain injury

4.4. Fatigue and sleep-disordered breathing in multiple sclerosis:

a clinically relevant association?

6. Acknowledgements 


\section{Introduction}

Wakefulness continuously alternates with sleep. While these two behavioral states may be regarded as mutually exclusive, they are at the same time closely interconnected and affect each other in a reciprocal way. Yet even after normal amount of consolidated sleep, any healthy individual experiences fluctuations in her or his wakefulness, encompassing moments when she or he enjoys full alertness, feels fatigued after sustained effort or struggles with drowsiness and increasing sleepiness. In other words, there is a continuous fluctuation among different states of vigilance, and in this cycle, periods of decreased arousal states can be regarded as physiological.

We take for granted the ability to be fully awake for about two-thirds of a 24-hour day. However, clinical practice conspicuously illustrates that impairment of normal wakefulness occurs in many disorders of the brain. In this context, decreased arousal states in the absence of sleep deprivation become pathological symptoms, as they are persistent, disabling and significantly impair quality of life.

Over the last two decades, neurologists have increasingly recognized the high prevalence, medicolegal consequences and considerable burden of arousal disturbances in neurological disorders. Despite this advanced awareness, arousal disturbances often remain underdiagnosed or inadequately assessed. The field remains a major challenge for both clinicians and researchers, because our understanding of the underlying neurobiology is still very limited, and - as a consequence - helping affected patients is frustrating due to a lack of treatment options targeting known neurophysiological deficits.

\section{Diagnostic approach to a frequent and disabling clinical problem}

As mentioned, recognition of arousal disturbances is compromised by multiple diagnostic difficulties. Several aspects highlight this problem and explain why arousal 
disturbances continue to be underrecognized in many neurological disorders. First, the lack of biomarkers or appropriate clinical features often hampers the objective assessment of arousal disturbances. This is particularly challenging for the problem of fatigue, which is a subjective experience that currently can be assessed only in a semi-quantitative manner by questionnaires. Second, different arousal disturbances may coexist in the same patient (e.g. sleepiness and fatigue), making it difficult for the treating physicians to differentiate them. Third, clinicians need a high degree of suspicion to diagnose arousal disturbances, because patients may not be aware of and thus not report them. Indeed, the regular use of objective tools such as the mean sleep latency test (MSLT) or actigraphy have revealed a considerable discrepancy between subjective and objective measures of arousal disturbances. ${ }^{1}$ Finally, different neurological disorders may produce similar results in sleep lab examinations (e.g. similar mean sleep latency in the MSLT), making the definition of additional features for differentiation necessary.

The following collection of papers illustrates the above-mentioned difficulties and provides potential approaches. In the first paper, we translated and validated the Fatigue Severity Scale (FSS), to offer both clinicians and researchers a tool for identification and quantification of fatigue. In the second paper, we demonstrated in patients with idiopathic Parkinson's disease that fatigue and excessive daytime sleepiness (EDS) are often overlapping yet in many aspects distinct arousal disturbances. In the third paper, we assessed sleep need following traumatic brain injury (TBI) and compared subjective estimates with actigraphic measures, thereby identifying a significant underestimation of effective sleep need by TBI patients. Eventually, in the fourth paper, we compared two patient groups with EDS - patients with narcolepsy and patients with behaviourally induced insufficient sleep syndrome (BIISS), which is the official term for chronic sleep deprivation and showed that analyzing the specific sequence of sleep stages in the MSLT may help differentiating these two conditions. 


\subsection{Validation of the Fatigue Severity Scale in a Swiss cohort}

(Valko PO, Bassetti CL, Bloch KE, Held U, Baumann CR. Validation of the Fatigue Severity Scale in a Swiss Cohort. Sleep 2008; 31:1601-1607)

Fatigue is a very common symptom in a large number of neurological disorders. ${ }^{2}$ It is a subjective experience that lacks a clear-cut definition but can be described as loss of energy, rapid exhaustion and physical and/or mental tiredness. While fatigue has been increasingly identified as a major cause for impaired health-related quality of life, the condition is nevertheless often underrecognized. A possible explanation is the lack of adequate tools to examine fatigue. Although several fatigue questionnaires have been introduced, they have been validated only in few disorders with inclusion of small patient numbers. ${ }^{3}$ Thus, in this study we aimed at validating the German version of the Fatigue Severity Scale, which is the most frequently used fatigue questionnaire. The FSS is a selfadministered questionnaire that contains 9 items and patients need to indicate on a Likert scale from 1 to 7 whether they agree or disagree with the statement. The final score is the mean value of the 9 items.

We obtained and compared complete FSS questionnaires of 454 healthy subjects and various patient groups, including 188 patients with multiple sclerosis, 235 patients who have suffered an ischemic stroke and 429 patients with different sleep-wake disorders. Linear regression analysis showed significantly lower values for healthy controls than each patient group: mean FSS scores were $3.00 \pm 1.08$ in healthy subjects, $4.66 \pm 1.64$ in patients with multiple sclerosis, $3.90 \pm 1.85$ in patients with previous ischemic stroke, and $4.34 \pm 1.64$ in patients with sleep-wake disorders (Figure 1). Considering the range of FSS scores in healthy controls and patients, we concluded that a value $\geq 4.00$ should be regarded as cut-off for the presence of fatigue. Using this cut-off, fatigue was present in $18 \%$ of healthy subjects, in $69 \%$ of patients with multiple sclerosis, in $49 \%$ of patients with previous ischemic stroke, and in $62 \%$ of patients with sleep-wake disorders. 
To explore the reliability of our German translation of the FSS, we performed an item analysis, examined the internal consistency, and assessed the test-retest variability. ${ }^{4,5}$ Cronbach's analysis exhibited a coefficient $\alpha$ of 0.93 for the entire sample, which reflects a high degree of internal consistency of the questionnaire. The lowest internal consistencies were found for items 1 and 2, but the internal consistency of the FSS did not improve when these two items were deleted. We assessed the test-retest variability in 104 healthy subjects and found no difference when repeating the testing after 21 days: FSS scores were $2.94 \pm$ 0.90 and $2.90 \pm 0.74(p=0.27)$.

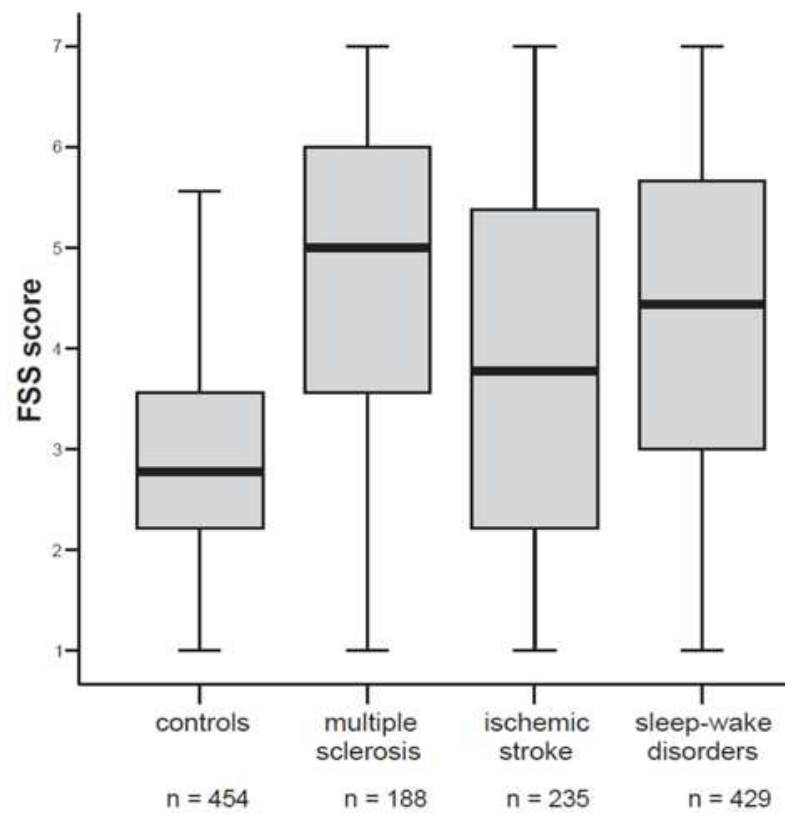

\section{Figure 1}

Boxplots demonstrating the median and interquartile range of FSS scores in healthy control subjects $(n=454)$, patients with multiple sclerosis $(n=188)$, patients with a history of ischemic stroke $(n=235)$, and patients with various sleep-wake disturbances $(n=429)$.

In conclusion, this was the first state-of-the-art validation of a fatigue scale in a large sample size $(n=1306)$ and for different neurological disorders. We demonstrated that the FSS is a valid instrument for detecting and monitoring fatigue and thus can be applied for both clinical and research purposes. 


\subsection{Fatigue and excessive daytime sleepiness in idiopathic Parkinson's disease differently correlate with motor symptoms, depression and dopaminergic treatment}

(Valko PO, Waldvogel D, Weller M, Bassetti CL, Held U, Baumann CR. Fatigue and excessive daytime sleepiness in idiopathic Parkinson's disease differently correlate with motor symptoms, depression and dopaminergic treatment. Eur $J$ Neurol 2010; 17:14281436)

Fatigue and EDS are both very common complaints in patients with idiopathic Parkinson's disease, and the reported prevalence ranges between $50-75 \%$ and $40-60 \%$, respectively. ${ }^{6-8}$ Many patients who suffer from EDS also feel fatigued, suggesting a high degree of clinical but also pathophysiological overlap of the two arousal disturbances. ${ }^{9}$ To explore the frequency of coexisting fatigue and EDS in Parkinson's disease and to identify potential differences in their association with other disease-related features, we set out to prospectively assess fatigue, EDS, depression, motor severity, disease duration, and dopaminergic treatment.

We included 88 consecutive patients with idiopathic Parkinson's disease from our Movement Disorders outpatient clinic. Using the Fatigue Severity Scale (FSS) and the Epworth Sleepiness Scale (ESS) with cut-off values of $\geq 4.00$ and $\geq 10$ for fatigue and EDS, respectively, we diagnosed fatigue in $59 \%$ and EDS in $48 \%$ of all patients, while an overlap of fatigue and EDS was observed in $35 \%$. FSS and ESS scores significantly correlated $(r=0.48, p<0.001)$, but the two arousal disturbances revealed different associations with other disease-related features. Fatigue significantly correlated with disease severity and motor disability, but also with the presence of autonomic disturbances and depression.
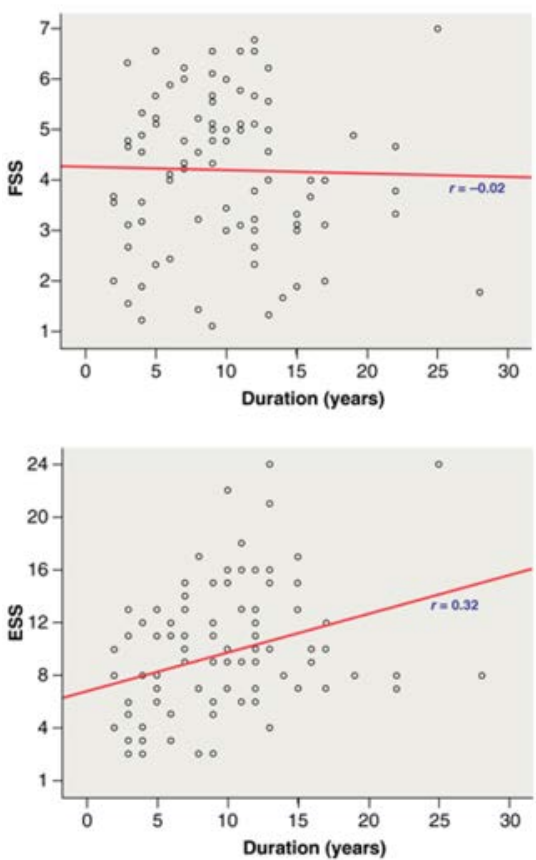

Figure 2

Correlation analysis between Parkinson's disease duration and scores of the Fatigue Severity Scale (FSS) and Epworth Sleepiness Scale (ESS). 
Conversely, EDS - but not fatigue significantly increased with longer disease duration (Figure 2).

Furthermore, dopaminergic treatment, namely the use of dopamine agonists, appeared to have a significant impact on the severity of EDS, but not on fatigue. Finally, using a multinomial regression model, we compared the frequency of depression between patients with fatigue alone, EDS alone, and co-occurrence of fatigue and EDS. As shown in Figure 3, only fatigue alone - as opposed to fatigue with EDS or EDS alone - was associated with a significant effect on depression.

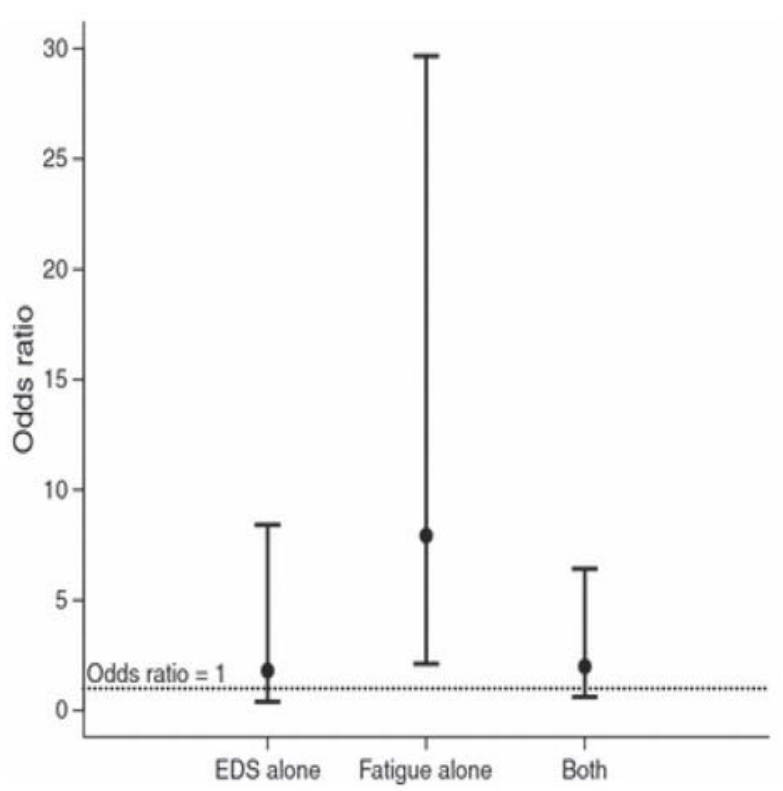

\section{Figure 3}

Multinomial regression model was used to assess the impact of depression on the odds ratio of only excessive daytime sleepiness (EDS; without fatigue), only fatigue (without EDS), and both symptoms. The graph illustrates the respective odds ratios and corresponding 95\% confidence intervals (Cl).

In conclusion, our study indicates that fatigue and EDS represent different constructs, as suggested by the observed disparities of their correlation to severity of motor symptoms, disease duration, depression, and dopaminergic treatment. The findings highlight the complex etiology especially of fatigue, which is probably influenced by a combination of primary and secondary factors, such as neurodegeneration of arousal-promoting brainstem and diencephalic structures, depression, sleep-wake disturbances including EDS and motor disability. 


\subsection{Excessive sleep need following traumatic brain injury: a case-control of 36 patients}

(Sommerauer M, Valko PO, Werth E, Baumann CR. Excessive sleep need following traumatic brain injury: a case-control study of 36 patients. J Sleep Res 2013; 22:634-639)

Arousal disturbances represent a major consequence in survivors of TBI. In a prospective study, posttraumatic EDS and increased sleep need (pleiosomnia) were identified as the most common sleep-wake disturbances. ${ }^{1}$ Posttraumatic hypersomnia is diagnosed if the patient persistently complains about increased sleep need of at least 2 hours per 24 hours as compared to the time prior TBI. The clinical impact and therapeutic implications of posttraumatic hypersomnia have not yet been elucidated, and thus represent the aim of this paper.

We conducted a case-control study of 36 TBI patients with posttraumatic pleiosomnia and compared the findings to 36 age- and sex-matched control subjects. We assessed subjective measures including fatigue, EDS and estimated sleep need per 24 hours by questionnaires and sleep logs. In addition, patients underwent also sleep laboratory recordings including 14-day actigraphy, polysomnography and MSLT.

Sleep increase after TBI ranged from 2.0 hours to 6.5 hours. Subjectively, TBI patients estimated their sleep need as 9.4 hours, but actigraphy revealed a significantly more pronounced sleep need of 10.8 hours $(p=0.02)$. Conversely, subjective estimation and actigraphic measurement of sleep need did not differ in control subjects ( 7.5 hours vs. 7.3 hours, respectively). In addition, $10 \mathrm{TBI}$ patients had an actigraphic pattern suggestive for chronic sleep deprivation, as indicated by longer sleep times of $\geq 2$ hours during weekend compared to weekday. All $10 \mathrm{TBI}$ patients returned to work.

Polysomnography demonstrated more slow-wave sleep in TBI patients than in control subjects $(20.4 \%$ vs. $13.8 \%, p=0.009)$, while all other parameters were similar. Finally, MSLT findings showed shorter mean sleep latency in TBI patients than in control subjects $(8.7 \mathrm{~min}$ vs. $12.4 \min , p<0.001)$. 
Thus, this paper suggests that posttraumatic pleiosomnia may be even more common than previously appreciated, because affected patients often do not estimate their actual sleep need correctly. The combination of pleiosomnia and underestimation or anosognosia of their posttraumatic sleep-wake disturbances may lead to an increased risk of secondary chronic sleep deprivation and excessive daytime sleepiness, particularly in TBI survivors returning to work. This finding should be considered when counseling and treating TBI patients, and must not be forgotten when dealing with the question whether a patient is able to drive motor vehicles.

\subsection{Multiple sleep latency measures in narcolepsy and behaviourally induced insufficient sleep syndrome}

(Marti I, Valko PO, Khatami R, Bassetti CL, Baumann CR. Multiple sleep latency measures in narcolepsy and behaviourally induced insufficient sleep syndrome. Sleep Med 2009; 10:1146-1150)

EDS is the mandatory symptom of narcolepsy. ${ }^{10,11}$ When narcolepsy patients do not present typical cataplexy, brief episodes of loss of postural muscle tone triggered by strong emotions, differentiating narcolepsy from other disorders with EDS may be difficult. Diagnostic criteria for narcolepsy include typical findings of the MSLT, such as a mean sleep latency of $\leq 8 \mathrm{~min}$ and the presence of $\geq 2$ sleep onset rapid eye movement (SOREM) periods. ${ }^{12}$ However, these MSLT markers are not specific and may also be present in patients with non-narcoleptic EDS, for instance due to sleep-disordered breathing or BIISS. ${ }^{13-}$ 17 To improve the diagnostic value of the MSLT in differentiating narcolepsy from patients with non-narcoleptic EDS, we analyzed MSLT data including sleep latencies to and between different sleep stages of 20 patients with narcolepsy with cataplexy, 20 BIISS patients and 20 age-, sex- and body mass index -matched control subjects.

We found that mean sleep latency to non-rapid eye movement (NREM) 1 was significantly shorter in narcolepsy $(1.8 \pm 1.5 \mathrm{~min})$ compared to BIISS $(4.7 \pm 2.1 \mathrm{~min} ; p<$ 
$0.001)$, but mean sleep latency to NREM2 was similar in narcolepsy and BIISS $(8.6 \pm 4.7 \mathrm{~min}$ vs. $8.1 \pm 2.7 \mathrm{~min})$. Three BIISS patients fulfilled the MSLT criteria for narcolepsy, and three narcolepsy patients had $\leq 1$ SOREM period. When analyzing only MSLT naps with SOREM periods, mean sleep latency from lights off to NREM1 were similar in narcolepsy and BIISS $(1.9 \pm 2.3 \mathrm{~min}$ vs. $1.8 \pm 1.3 \mathrm{~min}$ respectively; $p=0.96)$. Conversely, mean sleep latency from lights off to REM sleep was significantly shorter in narcolepsy than in BIISS $(5.4 \pm 3.8$ vs. $10.0 \pm 4.0 \mathrm{~min}$, respectively; $p<0.001)$. The reason for this discrepancy is that in narcolepsy the most common sleep stage sequence was NREM1 - REM - NREM2, while in BIISS it

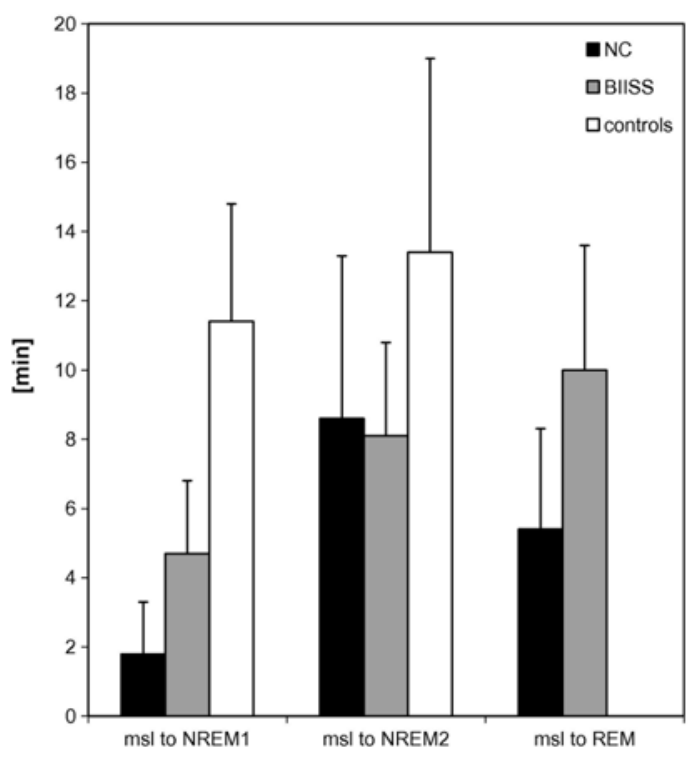

Figure 4

Group comparison of mean sleep latencies to different sleep stage. was NREM1 - NREM2 - REM (Figure 4).

The clinical presentation of narcolepsy and BIISS may show substantial overlap, and in the absence of typical cataplexy the differentiation of narcolepsy and BIISS can be difficult. A minority of BIISS patients (15\% in this paper) even fulfill the MSLT criteria for narcolepsy. For these diagnostic challenges, our study demonstrates that the sleep stage sequence NREM1 - REM - NREM2 may serve as additional discriminating factor (Figure 5).

\begin{tabular}{|c|c|c|c|c|c|}
\hline \multicolumn{3}{|c|}{ Sleep stage sequencing } & \multirow{2}{*}{$\frac{\text { NC }}{71 \%}$} & \multirow{2}{*}{$\frac{\text { B\|ISS }}{15 \%}$} & \multirow{2}{*}{$\begin{array}{c}\underline{p} \\
<0.001\end{array}$} \\
\hline NREM1 & REM & NREM2 & & & \\
\hline NREM1 & NREM2 & REM & $29 \%$ & $85 \%$ & $<0.001$ \\
\hline \multicolumn{3}{|c|}{ mean sleep latency } & & & \\
\hline & & & & & \\
\hline
\end{tabular}

\section{Figure 5}

Analysis of sleep stage sequence in MSLT naps with SOREMs (narcolepsy with cataplexy (NC): $n=62 / 85$ naps; BIISS: $n=13 / 89$ naps) reveals significant differences between NC and BIISS. 


\section{A neuropathological perspective}

One of the first clinico-anatomical studies that aimed at correlating specific sleepwake disturbances with structural lesions in the brain was done during the epidemic of encephalitis lethargica around the time of World War I. Von Economo reported that patients with lesions in the posterior part of the hypothalamus demonstrated profound EDS and increased sleep need. ${ }^{18} \mathrm{He}$ was probably the first to speculate that narcolepsy might represent a neurological disorder originating from the posterior hypothalamus. Seven decades later, when researchers discovered that narcolepsy is caused by an extensive loss of the hypothalamic neurons producing the hypocretin (also called orexin) neuropeptides, it became clear that von Economo was right. ${ }^{19-21}$

People with narcolepsy suffer from severe EDS, along with cataplexy, sleep paralysis, hypnagogic hallucinations and fragmented night-time sleep. The study of narcolepsy has proved crucial for our understanding of arousal disturbances in other neurological disturbances and continues to promote substantial advances in the field. Several groups examined the integrity of the neurons producing hypocretin in different neurological disorders commonly associated with chronic EDS, and found partial loss of these hypothalamic cells in postmortem brains of patients with idiopathic Parkinson's disease, ${ }^{22,23}$ Alzheimer's disease ${ }^{24}$ and Huntington's disease. ${ }^{25}$ These papers clearly represent an important contribution to our understanding why sleep-wake disturbances occur so frequently in neurodegenerative disorders. However, further studies are warranted to elucidate whether partial hypocretin deficiency is the principle etiology of arousal disturbances in neurodegenerative disorders which is probably not the case - and whether extensive hypocretin deficiency is solely responsible for all symptoms in narcolepsy with cataplexy.

As mentioned earlier, arousal disturbances represent a major and disabling symptom for a majority of patients surviving traumatic brain injury. A study in 1971 reported frequent hypothalamic lesions following closed head injury, ${ }^{26}$ but the hypothalamic arousal-promoting system including the neurons producing hypocretin was not known at that time and has not 
been investigated since. Thus, in the first paper of the second part of this habilitation treatise we compared the number of various hypothalamic neurons, including cells producing hypocretin and melanin-concentrating hormone $(\mathrm{MCH})$, between patients with fatal TBI and non-neurological control brains. In the second paper, we investigated the integrity of histamine neurons in narcolepsy brains. The histamine neurons are located in the tuberomammillary nucleus in the posterior hypothalamus, and they receive intense excitatory input from hypocretin neurons. ${ }^{27,28}$ The histaminergic system plays an important role in promoting wakefulness, but has not been examined yet in narcoleptic brains.

\subsection{Loss of hypocretin (orexin) neurons with traumatic brain injury}

(Baumann CR, Bassetti CL, Valko PO, Haybaeck J, Keller M, Clark E, Stocker R, Tolnay M, Scammell TE. Loss of hypocretin (orexin) neurons with traumatic brain injury. Ann Neurol 2009; 66:555-559)

Arousal disturbances such as EDS or increased sleep need (pleiosomnia) are common after traumatic brain injury (TBI) and may persist for many years. ${ }^{29-32}$ Roughly 1 in 4 TBI patients suffer from chronic EDS, as documented by several studies. Considering the common occurrence yet unclear etiology of posttraumatic sleepiness, we examined the hypothalami of 4 patients, who died after severe TBI. Previous studies had shown strongly reduced cerebrospinal fluid (CSF) concentration of hypocretin during the acute period after TBI, possibly reflecting functional down-regulation during coma or marked obtundation. ${ }^{33}$ Subsequently, however, this hypocretin deficiency was followed by recovery of CSF levels of hypocretin at 6 months after TBI. ${ }^{1}$ Interestingly, however, CSF levels of hypocretin did not return to normal values in all TBI patients, but remained reduced in some of them. ${ }^{1}$ Thus, we hypothesized that head trauma may cause damage to the hypothalamus, including partial loss of neurons producing hypocretin.

To test our hypothesis, we used immunohistochemistry for hypocretin-1 (orexin-A) and $\mathrm{MCH}$, counted the numbers of immunoreactive cells and performed quantitative 
comparison between 4 hypothalami from patients with fatal TBI and 4 control brains. As shown in Figure 6, we found a $27 \%$ reduction of neurons producing hypocretin in $\mathrm{TBI}$ brains compared to control brains $\left(23^{\prime} 655 \pm\right.$ 5'035 vs. $32^{\prime} 318 \pm$ 3’060; $p=$ 0.001). The number of $\mathrm{MCH}$ neurons also appeared to be lower in TBI brains than in control brains (39'540 \pm 10 '900 vs. $57^{\prime} 176$ \pm 24'016), without however reaching statistical significance $(p=0.06)$. Finally, immunostaining of glial fibrillary acidic protein (GFAP), a marker of astrocytes and reactive gliosis, revealed a higher density of reactivated astrocytes in TBI than control brains $\left(21 \pm 12 / \mathrm{mm}^{2}\right.$ vs. $7 \pm 2$ $/ \mathrm{mm}^{2}$ ), including dense perivascular immunoreactivity (Figure 7).
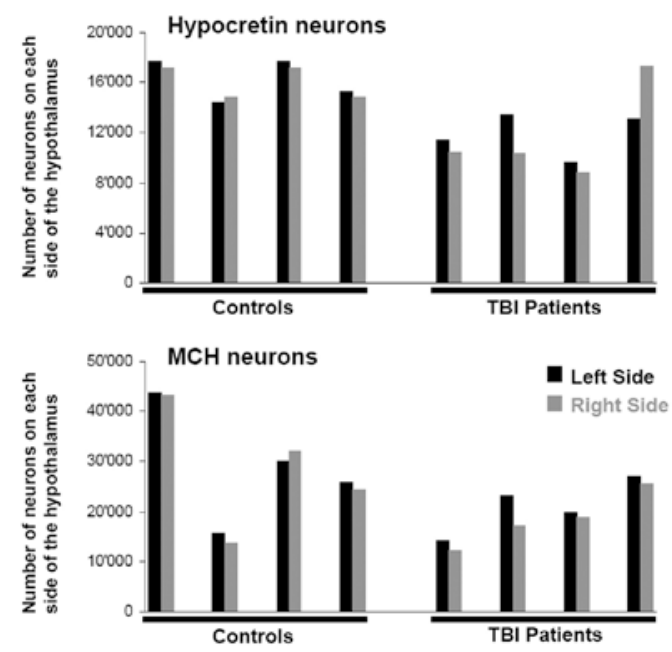

Figure 6

Quantitative comparison of neurons producing hypocretin and $\mathrm{MCH}$ in controls and TBI patients.

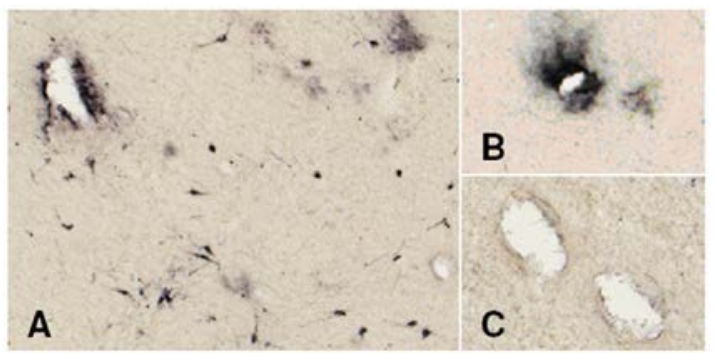

Figure 7

Immunostaining for hypocretin in a TBI brain, revealing dense perivascular immunoreactivity within the hypothalamic hypocretin field $(A, B)$, but not in adjacent areas (C).

This pilot study confirmed that head trauma is associated with a significant impact on the hypothalamus, as indicated by the reactive gliosis seen throughout the hypothalamus. Furthermore, hypothalamic injury involves a loss of several neuronal systems, including cells producing hypocretin and $\mathrm{MCH}$. Trauma-induced decrease of hypocretin signaling may thus contribute to the frequently observed posttraumatic arousal disturbances. Use of arousalstimulating drugs - ideally including hypocretin agonists - might represent a promising and tailored treatment option in patients with persistent posttraumatic arousal disturbances. 


\subsection{Increase of histaminergic tuberomammillary neurons in narcolepsy}

(Valko PO, Gavrilov YV, Yamamoto M, Reddy H, Haybaeck J, Mignot E, Baumann CR, Scammell TE. Increase of histaminergic tuberomammillary neurons in narcolepsy. Ann Neurol 2013; 74:794-804)

In the year 2000 , two groups discovered a $>90 \%$ loss of the number of hypothalamic neurons producing hypocretin in narcoleptic brains. ${ }^{19-21}$ It is assumed that the pathogenic attack to these cells occurs in a highly selective way, because the integrity of $\mathrm{MCH}$-producing neurons - the closest neighbors of the hypocretin neurons - appeared to be unaffected. However, other cell populations involved in the regulation of sleep and wakefulness have not been examined yet, and both cause and consequences of the hypocretin neuron loss in narcolepsy remain unknown.

Among the targets of the excitatory hypocretin neurons, the histaminergic tuberomammillary nucleus (TMN) in the posterior hypothalamus is considered to have a major role in maintaining normal wakefulness, as suggested by the fact that TMN neurons generally fire only after wakefulness is established. ${ }^{34-37}$ A large body of evidence suggests that the histamine system might play an important role in the neurobiology of narcolepsy. The TMN is the only brain structure producing histamine, receives strong excitatory innervation from hypocretin neurons, ${ }^{38-40}$ and histamine CSF levels have recently been reported to be decreased in narcolepsy and other conditions with excessive daytime sleepiness. ${ }^{41-43}$ In humans, centrally-acting antihistamines are known to elicit drowsiness. ${ }^{44}$ Eventually, while mice lacking histidine decarboxylase (HDC), a marker of the histaminergic TMN neurons, have less wakefulness at the beginning of their active period and sleep more in a novel environment, mice lacking histamine $\mathrm{H} 1$ receptors are not able anymore to show a normal arousal reaction to hypocretin.

To explore a potential involvement of the histamine system in the neurobiology of narcolepsy, we examined TMN neurons in human narcoleptic brains and in two mouse models of narcolepsy. In the human part, we included 7 brains of narcolepsy patients and 12 control brains, immunostained for hypocretin-1 (orexin-A), $\mathrm{HDC}$ and $\mathrm{MCH}$, and used 
unbiased stereology for quantification of positively labeled cells. For the rodent part we used 6 wild-type (WT) C57BL/6J mice, 5 prepro-orexin ligand knockout (KO) mice, and 6 orexin/ataxin-3 (Atx) transgenic mice. The latter express ataxin-3, which is a toxic transgene that selectively kills most of the hypocretin neurons by around 12 weeks of age.

We found a $94 \%$ increase of histaminergic TMN neurons in narcolepsy patients compared to controls, and the gain in histamine neurons was higher in those narcolepsy patients who had the greatest loss of neurons producing hypocretin (Figures $8+9)$. The pattern of rostro-caudal distributions of histaminergic neurons was similar in control subjects and narcolepsy patients (Figure 10).
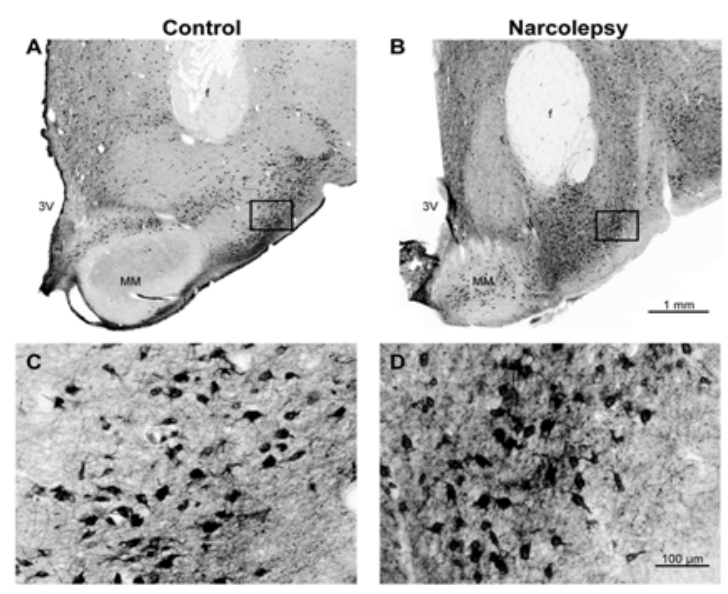

\section{Fiqure 8}

Histidine decarboxylase (HDC) immunostaining in control and narcolepsy subjects, showing approximately $60 \%$ more HDC-immunoreactive neurons in this section through the medial mammillary nucleus.
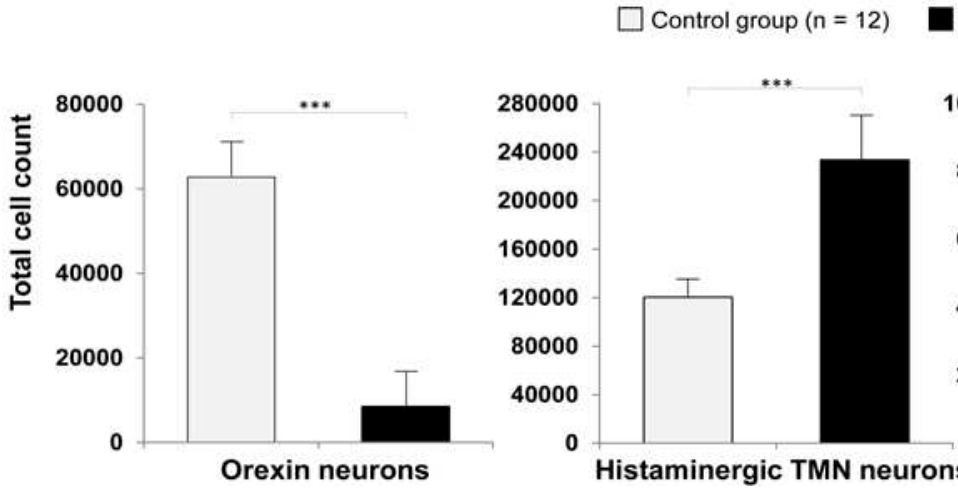

Narcolepsy ( $n=7)$

\section{Figure 9}

Patients with narcolepsy with cataplexy had $86 \%$ fewer hypocretin neurons $\left({ }^{* * *} p<0.001\right)$ and $94 \%$ more histamine neurons $\left({ }^{* *} p<0.001\right)$ than control subjects, while the number of $\mathrm{MCH}$ neurons was similar.

Likewise, hypocretin $\mathrm{KO}$ mice had 53\% more histaminergic TMN neurons, and Atx mice had $28 \%$ more histaminergic TMN neurons than WT mice (Figure 11). 


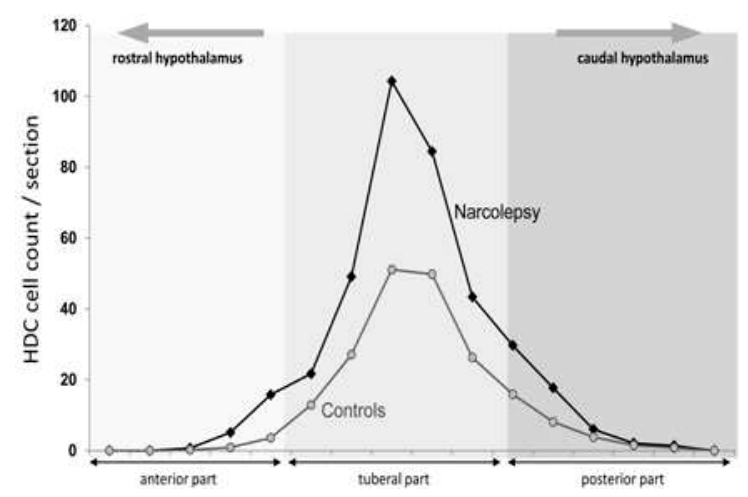

\section{Figure 10}

Similar pattern of rostro-caudal distributions of histaminergic tuberomammillary neurons in control subjects and narcolepsy patients.

Our discovery was surprising and raised several questions. Although our study does not provide a pathophysiologic explanation for the striking increase of histaminergic TMN neurons, we speculate this increment may reflect an effort to compensate for the reduction of excitatory hypocretin signaling. Furthermore, as not all symptoms occurring in the course of narcolepsy may be satisfactorily attributed to the loss of hypocretin neurons, the increase of histaminergic TMN neurons may account for the gradual decline of hypersomnia a few weeks or months after the onset of the disease, and the progressive sleep fragmentation, which usually develops later in the disease course than the appearance of sleepiness or cataplexy. Finally, drugs enhancing the histamine signaling may constitute an alternative treatment option for patients with narcolepsy or other neurological disorders with arousal disturbances.

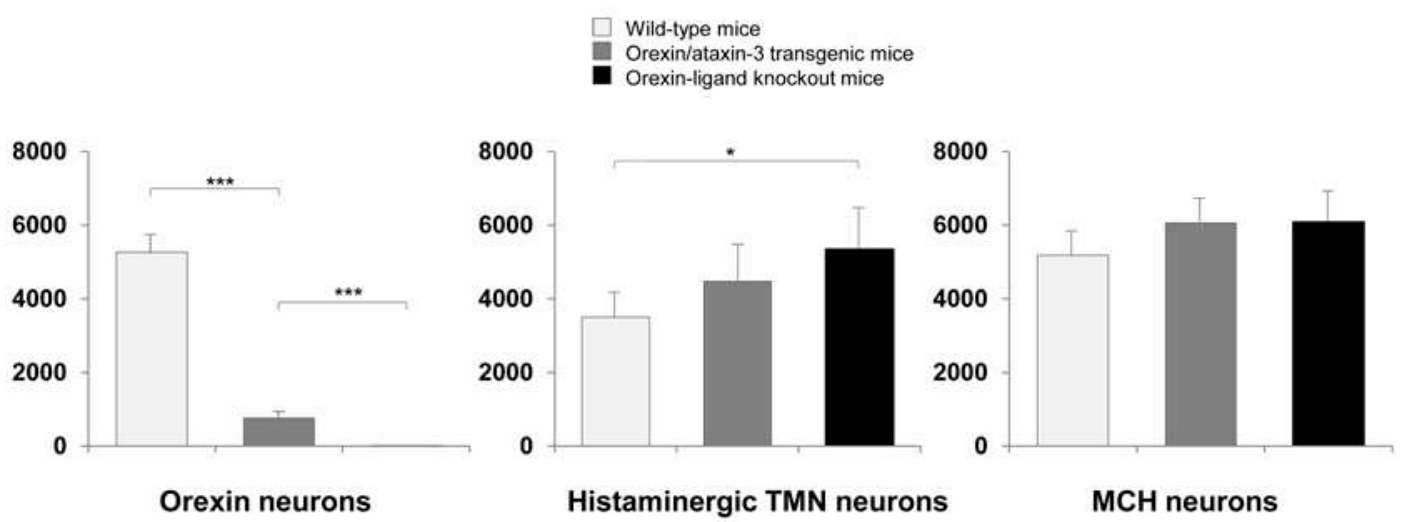

\section{Figure 11}

Cell counts in two mouse models of narcolepsy. Orexin-ligand knockout mice have no orexin immunoreactivity and 53\% more histaminergic tuberomammillary nucleus (TMN) neurons than wildtype mice. Orexin/ataxin-3 transgenic mice have an $85 \%$ loss of orexin neurons and $28 \%$ more histaminergic neurons than wild-type mice. The cell counts of neurons producing melaninconcentrating hormone $(\mathrm{MCH})$ is similar in all groups. 


\section{Therapeutic challenge}

In the last part of this habilitation treatise, I discuss different treatment strategies for arousal disturbances in neurological disorders. Considering the heterogeneous pathophysiology of arousal disturbances, opportunities of effective therapy may emerge from distinct approaches. Understanding of the underlying neurobiology of arousal disturbances should determine the choice of treatment. The best way, obviously, would be to prevent or treat the pathological process which is producing the arousal disturbance. We discuss this possibility in the first paper concerning the effect of intravenous immunoglobulins in patients with narcolepsy with cataplexy. The next two papers illustrate the common effort to alleviate arousal disturbances by the use of drugs that increase wakefulness. We demonstrate that this goal can be achieved in different manner, either by improving the quality of nocturnal sleep by using hypnotics such as sodium oxybate, or by reinforcing the activity of certain components of the ascending arousal system (although the precise mechanism of action remains uncertain, but the effect of modafinil might result from stimulation of wake-promoting histaminergic tuberomammillary neurons ${ }^{45}$ ). Finally, arousal disturbances may emerge not only as a direct consequence of impaired wake-promoting brain circuitries, but also by comorbidities that are associated with the underlying neurological disorder (e.g. depression or sleep disorders in multiple sclerosis). In the last paper, we discuss the relationship between sleep-disordered breathing and fatigue in patients with multiple sclerosis and whether treatment by continuous positive airway pressure (CPAP) significantly reduces fatigue severity.

\subsection{No persistent effect of intravenous immunoglobulins in patients with narcolepsy with cataplexy}

(Valko PO, Khatami R, Baumann CR, Bassetti CL. No persistent effect of intravenous immunoglobulins in patients with narcolepsy with cataplexy. J Neurol 2008; 255:1900-1903)

The cause of the hypocretin deficiency in narcolepsy is not understood, but several findings suggest an autoimmune etiology. First of all, there is no other neurological disorder 
exhibiting a similarly tight linkage to the human leukocyte antigen (HLA) system. Specifically, the HLA DQB1*0602 haplotype can be identified in $85-95 \%$ of all narcolepsy patients, whereas fewer than $25 \%$ of Caucasian American controls present this subtype allele. ${ }^{46,47}$ Furthermore, the impact of environmental factors, as demonstrated by the recent endemic increase of narcolepsy cases following the vaccination for the $\mathrm{H} 1 \mathrm{~N} 1$ influenza $\mathrm{A}$ virus, additionally favor the autoimmune hypothesis. ${ }^{48,49}$

Although the exact dynamics of the underlying pathogenic mechanism is poorly known, we currently assume injury to hypocretin neurons occurs over weeks to a few years. ${ }^{11}$ Obviously, the best treatment of narcolepsy would be to prevent it or, once the cardinal symptoms are emerging, to hamper full breakout by arresting the presumed and hopefully still incomplete attack to the hypocretin neurons. This is exactly what two groups claimed to have successfully accomplished by administering high-dose intravenous immunoglobulins (IVIg) to narcolepsy patients with hypocretin deficiency. ${ }^{50-52}$ The present study was undertaken to further evaluate the potential role of IVIg in the treatment of patients with narcolepsy with cataplexy.

Four patients with narcolepsy with cataplexy, aged 39-51 years, were treated with IVIg therapy. All patients had undetectable cerebrospinal fluid (CSF) hypocretin levels, and HLA-typing was positive for DQB $1{ }^{*} 0602$. In two patients, the latency between narcolepsy onset and IVIg trial was relatively short (4 and 11 months), while the other two patients have had narcolepsy for already 4 and 17 years. The IVIg trial consisted of three cycles at 5-week intervals, with a dose of $1 \mathrm{~g} / \mathrm{kg} /$ day administered over 2 days. At baseline and after each IVIg administration, we performed assessments of subjective and objective sleepiness using ESS, MSLT and maintenance of wakefulness test (MWT). In addition, the patients kept a diary on the frequency of cataplectic attacks.

In conclusion and in agreement with findings from other groups, we could not confirm persistent improvements of narcoleptic symptoms, although temporary effects could be observed. Thus, our report does not support the results of the first observations. We 
speculate that IVIg-related improvements may in part reflect a placebo effect, as suggested by the discrepant responses between objective measurements (MSLT, MWT) and subjective ratings (ESS score, cataplexy diary). Indeed, lack of difference between placebo and IVIg treatment was recently reported in a narcoleptic patient who underwent a double-blind, placebo-controlled IVIg trial. ${ }^{53}$

\subsection{Sodium oxybate in narcolepsy with cataplexy: Zurich sleep center experience}

(Poryazova R, Tartarotti S, Khatami R, Baumann CR, Valko P, Kallweit U, Werth E, Bassetti CL. Sodium oxybate in narcolepsy with cataplexy: Zurich sleep center experience. Eur Neurol 2011; 65:175-182)

The high clinical burden of narcolepsy is mainly defined by the severity of EDS and the frequency of cataplexy. However, disrupted night-time sleep, sleep paralysis and hypnagogic hallucinations may also be very annoying, unpleasant and frightening symptoms. In the absence of any definite cure, the current clinical management of narcolepsy patients relies on symptomatic treatment, usually combining stimulants (modafinil or amphetamine analogs) for EDS with tricyclic antidepressants for cataplexy. Sodium oxybate $\left(X y r e m^{\circledR}\right)$ is the only drug that potentially improves all narcoleptic symptoms, including not only EDS and cataplexy but also disturbed nocturnal sleep. ${ }^{54,55}$ Sodium oxybate is the sodium salt of $\gamma^{-}$ hydroxybutyrate and its action is believed to be mediated through GABA-B receptors. Large, randomized, double-blind, placebo-controlled studies confirmed the beneficial effects on all narcoleptic symptoms, mainly on cataplexy but also on EDS, albeit with higher dosages. ${ }^{56}$ The present work was undertaken to analyze our own experiences with sodium oxybate with regard to treatment effects and potential adverse events.

Overall, we included 18 HLA-DQB $1{ }^{*} 0602$ positive patients with narcolepsy with cataplexy. Hypocretin deficiency was confirmed in $17 / 17$ patients. The presence of both EDS and frequent cataplectic attacks constituted the indication for sodium oxybate therapy in all of them. To monitor the treatment effects, we compared ESS scores, frequency of cataplexy, sleep paralysis, hallucinations and nightmares, actigraphic sleep/rest efficiency, and the 
findings of polysomnography, MLST, MWT and steer-clear vigilance test before and after therapy with sodium oxybate. Mean dosage of sodium oxybate was $6.4 \pm 1.2 \mathrm{~g}$, and mean treatment duration was $26 \pm 13$ months.

We observed an improvement of all narcoleptic symptoms. Specifically, significant effects were found for ESS score (17.8 \pm 1 before and $13.9 \pm 1.2$ after treatment, $p=0.001$ ), number of cataplexy (20 vs. $1, p=0.001)$, number of sleep paralyses $(4.1 \pm 8.3$ vs. $0, p=$ $0.01)$, number of hallucinations $(7.6 \pm 9.5$ vs. $0.4 \pm 1.1, p=0.005)$, sleep/rest efficiency in actigraphy ( $60.5 \pm 19.7$ vs. $76 \pm 4, p=0.012$ ), mean sleep latency in MWT (5.5 \pm 4.5 vs. 17.4 $\pm 8.9, p<0.001)$, and error\% in SCVT $(10.8 \pm 12.5$ vs. $2.1 \pm 2.1, p=0.02)$. On the other hand, sodium oxybate was rather well tolerated. Nighttime confusion represented the most common adverse effect (33\%), followed by nausea (11\%), enuresis $(11 \%)$, diffuse muscle pain $(6 \%)$, headache $(6 \%)$, flatulence $(6 \%)$, and disturbance in attention and forgetfulness $(6 \%)$.

In conclusion, we confirmed that sodium oxybate effectively improves all narcoleptic symptoms. Since the drug is generally well tolerated, monotherapy with sodium oxybate should be considered as first-choice alternative to the combination of stimulants and tricyclic antidepressants.

\subsection{Modafinil ameliorates excessive daytime sleepiness after traumatic brain injury}

(Kaiser PR, Valko PO, Werth E, Thomann J, Meier J, Stocker R, Bassetti CL, Baumann CR. Modafinil ameliorates excessive daytime sleepiness after traumatic brain injury. Neurology 2010; 75:1780-1785)

Posttraumatic arousal disturbances are common, but specific treatment options for affected patients don't exist. A prospective study conducted in our department revealed a prevalence of fatigue and EDS of $17 \%$ and $38 \%$, respectively, among 65 consecutive TBI patients. ${ }^{1}$ In the present study, we set out to explore the effect of daily modafinil on 
posttraumatic fatigue and EDS. Modafinil is a wake-promoting drug approved by the US Food and Drug Administration for therapy of EDS occurring in narcolepsy, obstructive sleep apnea and shift work sleep disorder, but not yet in TBI patients complaining about chronic arousal disturbances.

We included 20 consecutive TBI patients with fatigue, EDS or both, and conducted a prospective, double-blind, randomized, placebo-controlled study (Figure 12, flow diagram).

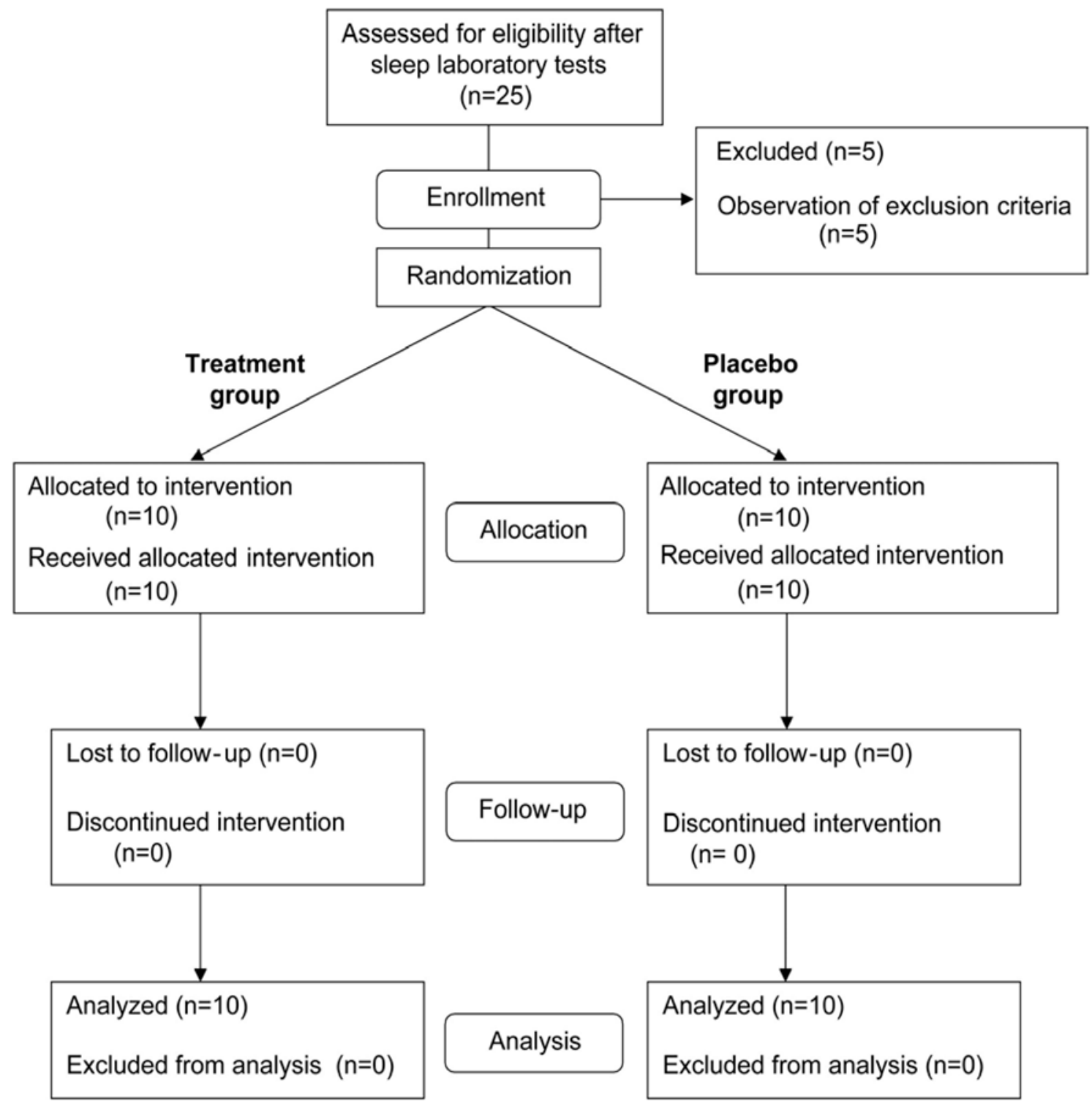

Figure 12 Flow diagram illustrating the passage of the participants through this trial. 
During 6 weeks, half of the TBI patients were treated with 100 to $200 \mathrm{mg}$ modafinil every morning, while the remaining half received placebo. We investigated the patients at baseline and after the treatment period as follows: fatigue and sleepiness were assessed by means of the FSS and ESS questionnaires, for evaluation of mood disturbances we applied the Beck Depression and Anxiety Inventory, and sleep laboratory examinations included 2week actigraphy, polysomnography, MWT, and psychomotor vigilance test.

Compared to the placebo group, ESS scores decreased and mean sleep latency on MWT increased to a significantly larger extent in the modafinil group (Figure 13). Improvement in ESS scores remained significant after correction for sex, age, TBI severity, and Beck Depression and Anxiety scale values. Conversely, multiple regression analysis revealed that the decrease in FSS score was not significantly higher in the modafinil group than in the placebo group.

In this trial, we showed that treatment with modafinil is effective in improving both subjective and objective posttraumatic EDS. The lack of significant effect on posttraumatic fatigue might be the result of underdosing, as modafinil is routinely given up to $400 \mathrm{mg}$ daily, or reflect distinct pathophysiology between sleepiness and fatigue.

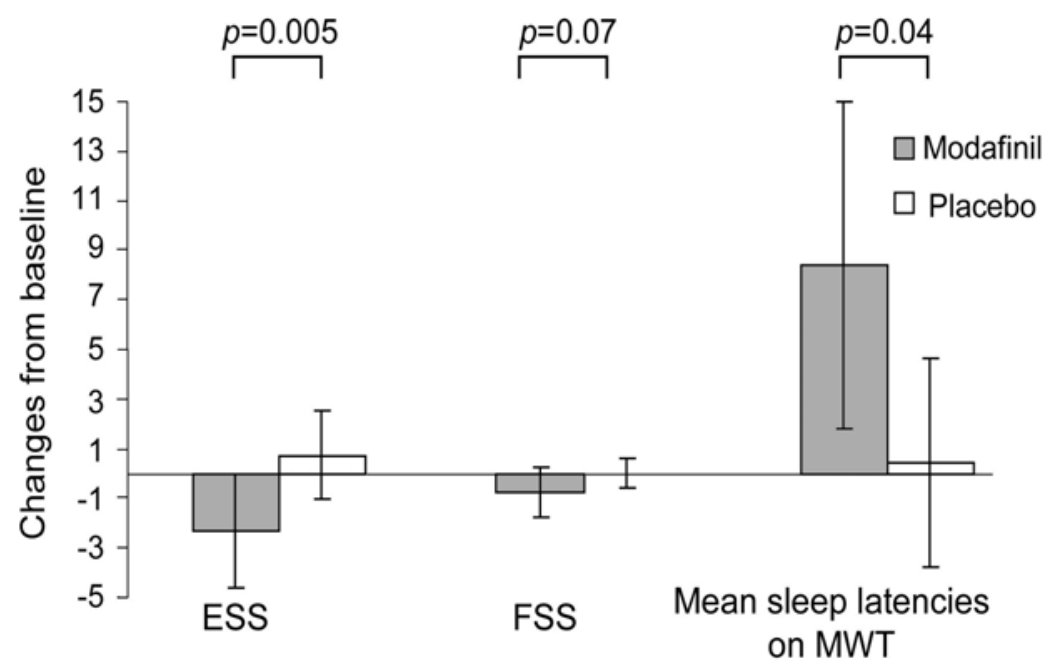

Figure 13 Changes from baseline in ESS and FSS scores, and in mean sleep latencies on MWT, between the modafinil and placebo groups. 


\subsection{Fatigue and sleep-disordered breathing in multiple sclerosis: a clinically relevant association?}

(Kallweit U, Baumann CR, Harzheim M, Hidalgo H, Pöhlau D, Bassetti CL, Linnebank M, Valko PO. Fatigue and sleep-disordered breathing in multiple sclerosis: a clinically relevant association? Mult Scler Int 2013:286581)

Fatigue is a very common complaint in patients with multiple sclerosis (MS) affecting up to $76-92 \%$, and is often perceived as one of the worst symptom. ${ }^{57,58}$ The etiology of fatigue in MS patients remains unclear. Presumably, the most important contributors to fatigue are disease-inherent features, i.e. the underlying neuroimmunological and neurodegenerative processes. While these factors are still poorly understood, additional and potentially treatable comorbidities such as depression, pain, anemia or sleep-wake disturbances must be identified..$^{59,60}$

Among these comorbidities, sleep-disordered breathing has recently been considered as a significant associate of fatigue in MS patients. ${ }^{61,62}$ In the present study we aimed at exploring whether respirography should be routinely conducted in MS patients with fatigue and whether treatment with continuous positive airway pressure (CPAP) reduced fatigue severity.

We prospectively screened 258 consecutive MS patients and diagnosed severe fatigue (FSS score $\geq 5.0)$ in $97(38 \%)$ patients. Respirography was eventually performed in 69/97 patients (71\%). SDB was diagnosed in 28 MS patients $(41 \%)$ and was mild (apneahypopnea index 5-15/h) in 18 patients (26\%), moderate (apnea-hypopnea index 15-30/h) in 3 patients (4\%), and severe (apnea-hypopnea index $\geq 30 / \mathrm{h}$ ) in 7 patients $(10 \%)$. CPAP therapy was recommended to 14 patients with and apnea-hypopnea index $\geq 10 / \mathrm{h}$, but only 6 patients consented and tolerated the device. As shown in Figure 14, after 6 months of CPAP treatment, we observed a mild yet significant reduction in FSS scores, while ESS scores remained unchanged. 

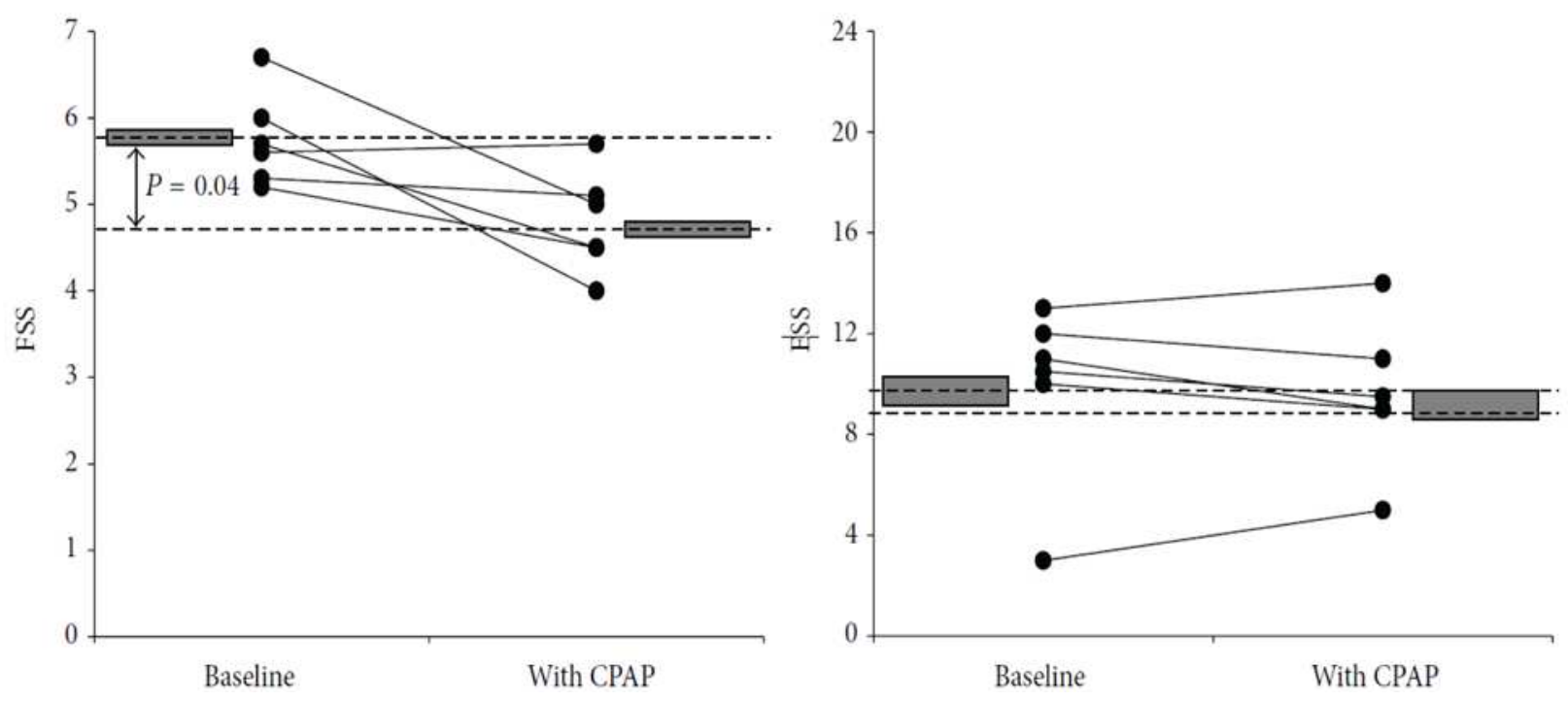

Figure 14 Follow-up after six months of CPAP treatment shows significant reduction of fatigue severity but not of sleepiness.

In conclusion, respirography should be considered in MS patients with fatigue, because the prevalence of sleep-disordered breathing is rather high. In addition, our study confirms previous reports of a significant reduction of fatigue after CPAP therapy, but the clinical relevance of this finding remains unclear, as (I) our treated group was very small, (II) not placebo-controlled, and (III) the impact on quality of life was not monitored. 


\section{Significance of the results}

The papers presented in this habilitation treatise highlight the significance of arousal disturbances in clinical practice of the neurologist. The importance of the topic relies on the unfavorable discrepancy between its high prevalence and arduous and far-reaching impact on quality of life on one hand, and the currently still marginal insights into the underlying neurobiology and our limited treatment options on the other hand. Combining clinical perspective and basic neuroscience, the results of the studies are of considerable relevance for both practicing neurologists and fundamental researchers.

In the first part, the author and his coworkers addressed common difficulties in the clinical and diagnostic assessment of arousal disturbances. The first validation study of the Fatigue Severity Scale in a large sample size now offers both clinicians and researchers a reliable, valid and very useful tool for detecting and monitoring fatigue. The remaining three studies exemplified different and highly characteristic challenges in the diagnosis of arousal disturbances, including overlap of distinct symptoms, discrepancy between subjective and objective measures, and differentiation of neurological disorders producing identical arousal disturbances.

The second part represents the centerpiece of our research, and illustrates how clinical observation has the potential to impact basic research. The discovery of quantitative changes in various components of the arousal-promoting system provides a significant contribution to our neurobiological understanding of arousal disturbances. Our report of a massive increase of histamine neurons in narcolepsy suggests the capability of the adult human brain to compensate for loss of a specific neuronal population. In addition to shedding light on the etiology of various sleep-wake disturbances, the implications of this result go beyond the mere study of arousal disturbances, as they give hope for the development of new treatment strategies in neurodegenerative disorders.

Finally, all coworkers of these studies hope that their neuroscientific research eventually contributes in turn to a better clinical and therapeutic management of affected 
patients. While the four studies of the third part indicate multiple approaches to improve arousal disturbances, they emphasize at the same time the necessity of further basic research to produce new and targeted treatment, which is based on neurobiological evidence.

\section{Acknowledgements}

Over the last decade, l've been lucky to receive continuous support by many colleagues and coworkers. First of all, I owe a great deal to my mentor PD Dr. Christian Baumann, who continues impressing me by his enthusiasm and scientific originality. He also enabled my postdoctoral fellowship in Boston, which appeared to be the most fruitful scientific period in my career so far. In this regard, I'm also particularly thankful to my American mentor, Prof. Dr. Thomas Scammell, who keeps providing generous and invaluable scientific support. I'm also grateful to Prof. Dr. Claudio Bassetti, my first clinical and scientific teacher in the field of sleep medicine. Finally, I would like to thank Prof. Dr. Michael Weller, head of the Department of Neurology in Zurich, for always encouraging my scientific plans and clinical career.

Finally, and most of all, I'm lovingly indebted to my parents Helena and Peter Valko, to my wife Yulia and my two children Azim und Guzal. 


\section{References}

1. Baumann CR, Werth E, Stocker R, Ludwig S, Bassetti CL. Sleep-wake disturbances 6 months after traumatic brain injury: a prospective study. Brain 2007; 130:1873-1883.

2. Chaudhuri A, Behan PO. Fatigue in neurological disorders. Lancet 2004; 363:978-988.

3. Krupp LB, LaRocca NG, Muir-Nash J, Steinberg AD. The fatigue severity scale. Application to patients with multiple sclerosis and systemic lupus erythematosus. Arch Neurol 1989; 46:1121-1123.

4. Lin L. A concordance correlation coefficient to evaluate reproducibility. Biometrics 1989; 45:255-268.

5. Cronbach LJ. Coefficient alpha and the internal structure of test. 16. Psychometrika 1951; 16:297-334.

6. Friedman $\mathrm{JH}$, Friedman $\mathrm{H}$. Fatigue in Parkinson's disease: a nine-year follow-up. Mov Disord 2001; 16: 1120-1122.

7. Rye DB, Bliwise DL, Dihenia B, Gurecki P. Daytime sleepiness in Parkinson's disease. J Sleep Res 2000; 9: 63-69.

8. Friedman JH, Chou KL. Sleep and fatigue in Parkinson's disease. Parkinsonism Relat Disord 2004; 10(Suppl.): 27-35.

9. Hossain JL, Ahmad P, Reinish LW, Kayumov L, Hossain NK, Shapiro CM. Subjective fatigue and subjective sleepiness: two independent consequences of sleep disorders? J Sleep Res 2005; 14:245-253.

10. Sturzenegger C, Bassetti CL. The clinical spectrum of narcolepsy with cataplexy: a reappraisal. J Sleep Res 2004; 13:395-406.

11. Dauvilliers Y, Arnulf I, Mignot E. Narcolepsy with cataplexy. Lancet 2007; 369:499-511.

12. American Academy of Sleep Medicine. International Classification of Sleep Disorders (ICSD2), diagnostic and coding manual, 2nd edn. Westchester, IL: American Academy of Sleep Disorders Association; 2005.

13. Mitler MM, Van den Hoed J, Carskadon MA, Richardson G, Guilleminault C, Dement WC. REM sleep episodes during the MSLT in narcoleptic patients. Electroenceph Clin Neurophysiol 1979; 46:479-481.

14. Aldrich MS, Chervin RD, Malow BA. Value of the multiple sleep latency test (MSLT) for the diagnosis of narcolepsy. Sleep 1997; 20:620-629.

15. Mignot E, Lin L, Finn L, Lopes C, Pluff K, Sundstrom ML, et al. Correlates of sleep-onset REM periods during the Multiple Sleep Latency Test in community adults. Brain 2006; 129:1609-1623. 
16. Guilleminault C, Mignot E, Partinen M. Controversies in the diagnosis of narcolepsy. Sleep $1994 ; 17: S 1-6$.

17. Chervin RD, Aldrich MS. Sleep onset REM periods during multiple sleep latency tests in patients evaluated for sleep apnea. Am J Respir Crit Care Med 2000; 161:426-431.

18. Von Economo C. Sleep as a problem of localization. J Nerv Ment Dis 1930; 71:249-259.

19. Peyron C, Faraco J, Rogers W, et al. A mutation in a case of early onset narcolepsy and a generalized absence of hypocretin peptides in human narcoleptic brains. Nat Med 2000; 6:991-997.

20. Thannickal TC, Moore RY, Nienhuis R, et al. Reduced number of hypocretin neurons in human narcolepsy. Neuron 2000; 27:469-474.

21. Nishino S, Ripley B, Overeem S, et al. Hypocretin (orexin) deficiency in human narcolepsy. Lancet 2000; 355:39-40.

22. Fronczek R, Overeem S, Lee SY, et al. Hypocretin (orexin) loss in Parkinson's disease. Brain 2007; 130:1577-1585.

23. Thannickal TC, Lai YY, Siegel JM. Hypocretin (orexin) cell loss in Parkinson's disease. Brain 2007; 130:1586-1595.

24. Fronczek R, van Geest S, Frölich M, et al. Hypocretin (orexin) loss in Alzheimer's disease. Neurobiol Aging 2012; 33:1642-1650.

25. Aziz A, Fronczek R, Maat-Schieman M, et al. Hypocretin and melanin-concentrating hormone in patients with Huntington disease. Brain Pathol. 2008; 18:474-483.

26. Crompton MR. Hypothalamic lesions following closed head injury. Brain 1971; 94:165-172.

27. Panula $\mathrm{P}$, Yang HY, Costa E. Histamine-concentrating neurons in the rat hypothalamus. Proc Natl Acad Sci USA 1984; 81:2572-2576.

28. Airaksinen MS, Paetau A, Paljärvi L, et al. Histamine neurons in human hypothalamus: anatomy in normal and Alzheimer diseased brains. Neuroscience 1991; 44:465-481.

29. Guilleminault C, Faull KF, Miles L, van den Hoed J. Posttraumatic excessive daytime sleepiness: a review of 20 patients. Neurology 1983; 33:1584-1589.

30. Castriotta RJ, Wilde MC, Lai JM, et al. Prevalence and consequences of sleep disorders in traumatic brain injury. J Clin Sleep Med 2007; 3:349 -356.

31. Stulemeijer M, van der Werf S, Bleijenberg G, Biert J, Brauer J, E Vos P. Recovery from mild traumatic brain injury: a focus on fatigue. J Neurol 2006; 253: 1041-1047.

32. Kempf J, Werth E, Kaiser PR, Bassetti CL, Baumann CR. Sleep-wake disturbances 3 years after traumatic brain injury. J Neurol Neurosurg Psychiatry 2010; 81:1402-1405. 
33. Baumann CR, Stocker R, Imhof HG, Trentz O, Hersberger M, Mignot E, Bassetti CL. Hypocretin-1 (orexin A) deficiency in acute traumatic brain injury. Neurology 2005; 65:147149.

34. Haas HL, Sergeeva OA, Selbach O. Histamine in the nervous system. Physiol Rev 2008; 88:1183-1241.

35. Ko EM, Estabrooke IV, McCarthy M, Scammell TE. Wake-related activity of tuberomammillary neurons in rats. Brain Res 2003; 992:220-226.

36. Lin JS. Brain structures and mechanisms involved in the control of cortical activation and wakefulness, with emphasis on the posterior hypothalamus and histaminergic neurons. Sleep Med Rev 2000; 4:471-503.

37. Takahashi K, Lin JS, Sakai K. Neuronal activity of histaminergic tuberomammillary neurons during wake-sleep states in the mouse. J Neurosci 2006; 26:10292-10298.

38. Peyron C, Tighe DK, van den Pol AN, et al. Neurons containing hypocretin (orexin) project to multiple neuronal systems. J Neurosci 1998; 18:9996-10015.

39. Eriksson KS, Sergeeva O, Brown RE, Haas HL. Orexin/hypocretin excites the histaminergic neurons of the tuberomammillary nucleus. J Neurosci 2001; 21:9273-9279.

40. Mochizuki T, Arrigoni E, Marcus JN, et al. Orexin receptor 2 expression in the posterior hypothalamus rescues sleepiness in narcoleptic mice. Proc Natl Acad Sci USA 2011; 108:4471-4476.

41. Nishino S, Sakurai E, Nevsimalova S, et al. Decreased CSF histamine in narcolepsy with and without low CSF hypocretin-1 in comparison to healthy controls. Sleep 2009; 32:175-180.

42. Kanbayashi $\mathrm{T}$, Kodama $\mathrm{T}$, Kondo $\mathrm{H}$, et al. CSF histamine contents in narcolepsy, idiopathic hypersomnia and obstructive sleep apnea syndrome. Sleep 2009; 32:181-187.

43. Bassetti CL, Baumann CR, Dauvilliers $Y$, et al. Cerebrospinal fluid histamine levels are decreased in patients with narcolepsy and excessive daytime sleepiness of other origin. $J$ Sleep Res 2010; 19:620-623.

44. Roehrs TA, Tietz El, Zorick FJ, Roth T. Daytime sleepiness and antihistamines. Sleep 1984; 7:137-141.

45. Ishizuka T, Murakami M, Yamatodani A. Involvement of central histaminergic systems in modafinil-induced but not methylphenidate-induced increases in locomotor activity in rats. Eur J Pharmacol 2008; 578:209-215.

46. Rogers AE, Meehan J, Guilleminault C, Grumet FC, Mignot E. HLA DR15 (DR2) and DQB1*0602 typing studies in 188 narcoleptic patients with cataplexy. Neurology 1997; 48:1550-1556.

47. Mignot E, Lin L, Rogers W, et al. Complex HLA-DR and -DQ interactions confer risk of narcolepsy-cataplexy in three ethnic groups. Am J Hum Genet 2001; 68:686-699. 
48. Partinen M, Saarenpää-Heikkilä O, Ilveskoski I, et al. Increased incidence and clinical picture of childhood narcolepsy following the $2009 \mathrm{H} 1 \mathrm{~N} 1$ pandemic vaccination campaign in Finland. PLoS One 2012; 7:e33723.

49. Nohynek $\mathrm{H}$, Jokinen $\mathrm{J}$, Partinen $\mathrm{M}$, et al. AS03 adjuvanted $\mathrm{AH} 1 \mathrm{~N} 1$ vaccine associated with an abrupt increase in the incidence of childhood narcolepsy in Finland. PLoS One 2012; 7:e33536.

50. Lecendreux M, Maret S, Bassetti C, Mouren M-C, Tafti M. Clinical efficacy of high-dose intravenous immunoglobulins near the onset of narcolepsy in a 10-year-old boy. J Sleep Res 2003; 12:347-348.

51. Dauvilliers Y, Carlander B, Rivier F, Touchon J, Tafti M. Successful management of cataplexy with intravenous immunoglobulins at narcolepsy onset. Ann Neurol 2004; 56:905-908.

52. Dauvilliers Y. Follow-up of four narcolepsy patients treated with intravenous immunoglobulins. Ann Neurol 2006; 60:153.

53. Fronczek R, Verschuuren J, Lammers GJ. Response to intravenous immunoglobulins and placebo in a patient with narcolepsy with cataplexy. J Neurol 2007; 254:1607-1608.

54. Lammers GJ, Bassetti C, Billiard $M$ et al: Sodium oxybate is an effective and safe treatment for narcolepsy. Sleep Med 2010; 11:105-106.

55. Scammell TE. Modafinil: mechanisms of action. In: Bassetti CL, Billiard M, Mignot E, eds. Narcolepsy and Hypersomnia. New York, NY: Informa Healthcare; 2007:547:559.

56. US Xyrem ${ }^{\circledR}$ Multicenter Study Group: A randomized, double-blind, placebo-controlled multicenter trial comparing the effects of three doses of orally administered sodium oxybate with placebo for the treatment of narcolepsy. Sleep 2002; 25:42-49.

57. Branas $P$, Jordan R, Fry-Smith $A$, et al. Treatments for fatigue in multiple sclerosis: a rapid and systematic review. Health Technol Assess 2000; 4:1-61.

58. Zifko UA. Management of fatigue in patients with multiple sclerosis. Drugs $2004 ; 64: 1295-$ 1304.

59. Tachinana N, Howard RS, Hursch NP, Miller DH, Moseley IF, Fish D. Sleep problems in multiple sclerosis. Eur Neurol 1994; 34:320-323.

60. Mills RJ, Young CA. The relationship between fatigue and other clinical features of multiple sclerosis. Mult Scler 2011; 17:604-612.

61. Braley TJ, B. M. Segal BM, and R. D. Chervin RD. Sleep-disordered breathing in multiple sclerosis. Neurology 2012; 79:929-936.

62. Kaminska M, Kimoff RJ, A. Benedetti A, et al. Obstructive sleep apnea is associated with fatigue in multiple sclerosis. Mult Scler 2012; 18:1159-1169. 
Paper appendix 


\title{
Validation of the Fatigue Severity Scale in a Swiss Cohort
}

\author{
Philipp O. Valko, MD'; Claudio L. Bassetti, MD'; Konrad E. Bloch, MD²; Ulrike Held, PhD³; Christian R. Baumann, MD
}

${ }^{1}$ Department of Neurology, ${ }^{2}$ Pulmonary Division, and ${ }^{3}$ Horton Centre for Patient-Oriented Research, University Hospital of Zurich, Zurich, Switzerland

Background: Fatigue is highly prevalent and has a negative impact on quality of life and performance in a variety of disorders. The 9-item Fatigue Severity Scale (FSS) is one of the most commonly used selfreport questionnaires to measure fatigue, but has only been validated in small sample-sized studies and in single disorders.

Objective: To validate the FSS in healthy subjects and different disorders known to be commonly associated with fatigue.

Material and Methods: The FSS was administered to 454 healthy subjects, 188 patients with multiple sclerosis (MS), 235 patients with recent ischemic stroke, and 429 patients with sleep-wake disorders including narcolepsy with cataplexy $(n=22)$, restless legs syndrome $(R L S)(n=$ $79)$, sleep apnea $(n=108)$, insomnia $(n=62)$, parasomnia $(n=25)$, excessive daytime sleepiness/hypersomnia of other origin $(n=84)$, and other sleep-wake disorders $(n=49)$.
Results: FSS scores were $4.66 \pm 1.64$ (mean \pm SD) in patients with MS, $3.90 \pm 1.85$ in patients after ischemic stroke, and $4.34 \pm 1.64$ in patients with sleep-wake disorders. Compared to patients, values were significantly lower in healthy subjects $(3.00 \pm 1.08, P<0.01)$. Scores did not correlate with gender, age, or education. Item analysis showed an excellent internal consistency and reliability (Cronbach $a=0.93$ ). Test-retest variability was assessed in 104 healthy subjects, showing stable values over time ( $2.94 \pm 0.90$ vs. $2.90 \pm 0.74 ; P=0.27)$.

Conclusions: This first validation of a fatigue scale in a large sample size demonstrates that the FSS is a simple and reliable instrument to assess and quantify fatigue for clinical and research purposes.

Keywords: Fatigue, sleep, multiple sclerosis, validity, reliability Citation: Valko PO; Bassetti CL; Bloch KE; Held U; Baumann CR. Validation of the fatigue severity scale in a swiss cohort. SLEEP 2008;31(11):1601-1607.
FATIGUE CAN BE DEFINED AS A SUBJECTIVE EXPERIENCE, AND INCLUDES SUCH SYMPTOMS AS RAPID INANITION, PERSISTING LACK OF ENERGY, EXHAUSTION, physical and mental tiredness, and apathy. ${ }^{1}$ It can be a consequence of many sleep-wake disorders, but also of a large variety of other disorders including multiple sclerosis (MS) (present in as many as $76 \%$ to $92 \%$; experienced as worst symptom by $50 \%$ to $60 \%$ of patients), and stroke (poststroke fatigue, up to $68 \%) .{ }^{2-10}$ Fatigue represents one of the most frequent complaints of primary care patients $(6 \%$ to $45 \%){ }^{6}$

The large number of publications on fatigue in the last decade reflects the increasing awareness and the substantial role it has gained in clinical practice and research. In spite of this increasing interest in fatigue, as well as its prevalence and clinical significance, fatigue is underrecognized, possibly because of the lack of sufficiently validated and widespread instruments to quantify fatigue. Numerous fatigue scales have been introduced; only few of them have been validated, mostly in small studies and for specific disorders. ${ }^{11,12}$ Hence, the availability of an appropriate tool for assessment and quantification of fatigue, which can be used in different disorders, is important for clinical and research purposes.

A recent bibliographic study of fatigue measurement scales has shown that the FSS is the most commonly used fatigue specific questionnaire. ${ }^{13}$ The Fatigue Severity Scale (FSS) is a 9-item self-report questionnaire scale developed in 1989; it was applied in $25 \mathrm{MS}$ patients, 29 patients with systemic lupus erythematosus, and in 20 healthy controls. ${ }^{11}$ The simple and time-

Submitted for publication December, 2007

Accepted for publication June, 2008

Address correspondence to: Christian R. Baumann, MD, Neurology Department, University Hospital of Zurich, 8091 Zurich, Switzerland; Tel: +41 4425555 11; Fax: +41 4425543 80; E-mail: christian.baumann@usz.ch saving application of the FSS is probably the main reason for its high general acceptance. However, its use has been mainly limited to MS patients; large studies including patients with other sleep-wake and neurological disorders are lacking. These limitations may be the reason that there is no clearly defined FSS cut-off to discriminate normal from pathological results. ${ }^{1}$

Our aim in this study was to validate the FSS for the first time in a large sample size, by applying it to healthy controls and selected disorders frequently associated with fatigue.

\section{MATERIAL AND METHODS}

The study was conducted at the Neurology and Pulmonary Departments of the University Hospital of Zurich, Switzerland, between December 2005 and April 2007. The study protocol was approved by the local ethics committee.

\section{German Translation of the FSS}

The FSS is a self-administered questionnaire with 9 items (questions) investigating the severity of fatigue in different situations during the past week. Grading of each item ranges from 1 to 7 , where 1 indicates strong disagreement and 7 strong agreement, and the final score represents the mean value of the 9 items. We initially translated the 9 items of the FSS from English into German. Thereafter, a bilingual neurologist who was blinded with respect to the original version translated it backwards into English. Finally, the German version was adapted according to this procedure (Figure 1).

\section{Subjects}

We included 3 different patient groups (MS, ischemic stroke, and sleep-wake disorders), in which the prevalence of fatigue is 
Fatigue Severity Scale (FSS, English version)*

\begin{tabular}{|c|c|c|c|c|c|c|c|}
\hline & \multicolumn{3}{|c|}{$\begin{array}{l}\text { strongly } \\
\text { disagree }\end{array}$} & & & & \multirow{2}{*}{$\begin{array}{c}\text { strongly } \\
\text { agree }\end{array}$} \\
\hline & 1 & 2 & 3 & 4 & 5 & 6 & \\
\hline 1. My motivation is lower when I am fatigued. & 0 & 0 & 0 & 0 & 0 & 0 & 0 \\
\hline 2. Exercise brings on my fatigue. & 0 & 0 & 0 & 0 & 0 & 0 & 0 \\
\hline 3. I am easily fatigued. & 0 & 0 & 0 & 0 & 0 & 0 & 0 \\
\hline 4. Fatigue interferes with my physical functioning. & 0 & 0 & 0 & 0 & 0 & 0 & 0 \\
\hline 5. Fatigue causes frequent problems for me. & 0 & 0 & 0 & 0 & 0 & 0 & 0 \\
\hline 6. My fatigue prevents sustained physical functioning. & 0 & 0 & 0 & 0 & 0 & 0 & 0 \\
\hline $\begin{array}{l}\text { 7. Fatigue interferes with carrying out certain duties } \\
\text { and responsibilities. }\end{array}$ & 0 & 0 & 0 & 0 & 0 & 0 & 0 \\
\hline 8. Fatigue is among my three most disabling symptoms. & 0 & 0 & 0 & 0 & 0 & 0 & 0 \\
\hline 9. Fatigue interferes with my work, family, or social life. & 0 & 0 & 0 & 0 & 0 & 0 & 0 \\
\hline
\end{tabular}

\section{Fatigue Severity Scale (FSS, German version)}

\section{Ich finde, dass während der vergangenen Woche folgendes zutraf:}

1. Ich bin weniger motiviert, wenn ich müde bin.

2. Körperliche Bewegung macht mich müde.

3. Ich ermüde rasch.

4. Meine Müdigkeit beeinträchtigt meine körperliche Leistungsfähigkeit.

5. Meine Müdigkeit bereitet mir oft Probleme.

6. Meine Müdigkeit verhindert längerdauernde körperliche Tätigkeiten.

7. Meine Müdigkeit beeinträchtigt mich, gewisse Pflichten und Verantwortungen zu erfüllen.

8. Meine Müdigkeit gehört zu den drei Beschwerden, die mich am meisten behindern.

9. Meine Müdigkeit beeinträchtigt meine Arbeit, meine Familie oder mein soziales Leben.

\section{Stimme gar \\ nicht zu}

Stimme voll-

kommen zu

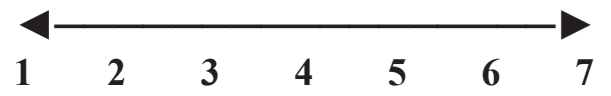

$\begin{array}{lllllll}0 & 0 & 0 & 0 & 0 & 0 & 0 \\ 0 & 0 & 0 & 0 & 0 & 0 & 0 \\ 0 & 0 & 0 & 0 & 0 & 0 & 0 \\ 0 & 0 & 0 & 0 & 0 & 0 & 0 \\ 0 & 0 & 0 & 0 & 0 & 0 & 0 \\ 0 & 0 & 0 & 0 & 0 & 0 & 0 \\ 0 & 0 & 0 & 0 & 0 & 0 & 0 \\ 0 & 0 & 0 & 0 & 0 & 0 & 0 \\ 0 & 0 & 0 & 0 & 0 & 0 & 0\end{array}$

Figure 1-The original English and the German version of the Fatigue Severity Scale (FSS). 
Table 1-Demographic and Clinical Characteristics of Healthy Subjects and Patients in this Study

\begin{tabular}{|c|c|c|c|c|}
\hline & $\begin{array}{l}\text { Healthy subjects } \\
\qquad(n=454)\end{array}$ & $\begin{array}{l}\text { Multiple sclerosis } \\
\qquad(\mathbf{n}=\mathbf{1 8 8})\end{array}$ & $\begin{array}{l}\text { Previous ischemic stroke } \\
\qquad(\mathrm{n}=\mathbf{2 3 5})\end{array}$ & $\begin{array}{l}\text { Sleep-wake disorders } \\
\qquad(\mathrm{n}=\mathbf{4 2 9})\end{array}$ \\
\hline Age (years, mean $\pm \mathrm{SD}$, range) & $47 \pm 18(13-94)$ & $45 \pm 13(20-79)$ & $63 \pm 14(21-87)$ & $52 \pm 15(16-86)$ \\
\hline Gender: female $(\%)$ & 60 & 67 & 31 & 35 \\
\hline \multicolumn{5}{|l|}{ Education status, n (\%) } \\
\hline Primary school degree & $43(10)$ & $63(36)$ & $59(28)$ & $80(28)$ \\
\hline Second school degree & $128(29)$ & $57(32)$ & $105(49)$ & $113(40)$ \\
\hline College degree & $93(21)$ & $26(15)$ & $21(10)$ & $34(12)$ \\
\hline University degree & $183(41)$ & $30(17)$ & $29(14)$ & $59(21)$ \\
\hline $\begin{array}{l}\text { Duration since disease onset } \\
\text { (years, mean } \pm \mathrm{SD} \text {, range) }\end{array}$ & & $\begin{array}{c}11.07 \pm 9.79 \\
(0.5-58)\end{array}$ & $\begin{array}{l}1.21 \pm 0.62 \\
(0.33-2)\end{array}$ & \\
\hline EDSS $($ mean \pm SD) & & $3.61 \pm 2.26$ & & \\
\hline VAS fatigue (mean $\pm \mathrm{SD}$ ) & $3.47 \pm 2.24$ & $4.83 \pm 2.49$ & $4.65 \pm 2.55$ & $5.12 \pm 2.45$ \\
\hline FSS score, mean $\pm \mathrm{SD}$ & $3.00 \pm 1.08$ & $4.66 \pm 1.64$ & $3.90 \pm 1.85$ & $4.34 \pm 1.64$ \\
\hline $95 \% \mathrm{CI}$ & $2.90-3.10$ & $4.42-4.89$ & $3.66-4.14$ & $4.19-4.50$ \\
\hline FSS score $\geq 4.0$ & $18 \%$ & $69 \%$ & $49 \%$ & $62 \%$ \\
\hline
\end{tabular}

SD: Standard Deviation, EDSS: Expanded Disability Status Scale, VAS: Visual Analog Scale, FSS: Fatigue Severity Scale, CI: Confidence Interval

known to be high. We sent the FSS by postal service to 314 patients with clinically definite MS and to 490 patients with previous ischemic stroke; these consecutive patients were examined in our neurological clinic between January 2005 and January 2007. A total of 188 patients (60\%) with MS and 235 patients (48\%) with ischemic stroke filled in and returned the questionnaire. The intervals between disease onset and the completion of this study were $11.1 \pm 9.8$ (mean $\pm \mathrm{SD}$, range $0.5-58$ ) years for MS patients and $1.2 \pm 0.6$ (range 0.33-2) years for stroke patients. In addition, we prospectively included 429 consecutive sleep-wake disordered patients referred to our neurological $(\mathrm{n}=345)$ and pulmonary $(\mathrm{n}=84)$ sleep clinics since December 2005. These patients were diagnosed with narcolepsy with cataplexy $(n=22)$, RLS $(n=79)$, sleep apnea $(n=108)$, insomnia $(n=62)$, parasomnia $(n=25), E D S /$ hypersomnia of other origin $(n=84)$, and other sleep-wake disorders $(n=49)$.

The FSS was also administered to 454 healthy control subjects recruited by the authors among relatives and friends. We attempted to obtain a control group that was representative of the general population with an equal distribution of different age categories and educational status (defined as highest educational degree attained). Subjects with a diagnosed sleep-wake disorder, previous sleep studies, or other diseases known to cause fatigue (e.g., advanced cancer, HIV infection, heart failure, rheumatic disorders and depression) were excluded.

\section{Questionnaire}

Apart from the FSS, all subjects had to quantify their fatigue on a Visual Analog Scale (VAS), where 0 indicates "very alert" and 10 "extremely fatigued." Furthermore, the German version of the Epworth Sleepiness Scale (ESS) and questions regarding bedtime were simultaneously administered. ${ }^{14}$

\section{Statistical Methods}

We used SPSS (version 12.0), STATA (version 9), and R (version 2.4.1) for statistical analysis. Group data are described by means and standard deviations (SD). We calculated comparisons between patient groups by means of a linear regression model, including thorough residual analysis. We performed correlation analyses between FSS and continuous demographic variables with the Pearson correlation coefficient; for binary comparisons we used the $t$-test. We considered statistical test results to be significant at a level of $\mathrm{P}<0.05$. Reliability was estimated through stability (test-retest) and internal consistency assessment. The test-retest variability of the scale was evaluated by Lin's correlation coefficient of concordance, ${ }^{15}$ which takes departures from the $45^{\circ}$ angle bisector into account, and which is typically lower than the corresponding correlation coefficient of Pearson. Internal consistency of the FSS was assessed by calculating the item to total correlation and the Cronbach $\alpha$ statistics. ${ }^{16}$ Cronbach $\alpha$ measures how well a set of items (or variables) measures a single unidimensional latent construct. In our case, $\alpha$ reflected how the 9 items of the FSS scale measured overall fatigue in patients or healthy subjects.

\section{RESULTS}

\section{Subjects}

The demographic and clinical characteristics of the patient groups and the control group are listed in Table 1.

\section{FSS: Comparison between Groups}

Mean FSS scores were $4.66 \pm 1.64$ in MS patients $(\geq 4.0$ in $69 \%$ ), $3.90 \pm 1.85$ in patients with previous ischemic stroke ( $\geq 4.0$ in $49 \%$ ), $4.34 \pm 1.64$ in patients with sleep-wake disorders ( $\geq 4.0$ in $62 \%)$, and $3.00 \pm 1.08$ in healthy controls ( $\geq 4.0$ in $18 \%$ ). The values for $95 \%$ confidence intervals (CI) are shown in Table 1 . The results of the linear regression analysis showed a significantly higher FSS score for each of the 3 patient groups than healthy controls; the effects of each of the 4 groups on mean FSS can be found in Table 2. The residual analysis revealed symmetrically distributed residuals around zero, and 
Table 2-Results of the Linear Regression Model: Estimated Effects and Standard Errors of the 4 groups (Healthy Subjects, Patients with Sleep-Wake Disorder, MS, and Previous Ischemic Stroke) on the Mean FSS Score

\section{Coefficient}

Healthy subjects Sleep-wake disorder

Multiple sclerosis

Ischemic stroke

$\begin{array}{ccc}\text { Estimate } & \text { Standard Error } & \begin{array}{c}\text { P-value } \\ 3.00\end{array} \\ 4.34 & 0.07 & <0.01 \\ 4.66 & 0.07 & <0.01 \\ 3.90 & 0.10 & <0.01 \\ & & <0.01\end{array}$

only a minor departure from the model assumptions, as the distribution of the residuals showed a small ceiling and bottom effect. This means that slightly more observations lie in the far left and far right side of the histogram of the residuals than one would expect under a strict Gaussian distribution.

\section{Normal Range and Cutoff of the FSS}

Mean \pm 2 SD of FSS scores in healthy controls ranged from 0.8-5.2. When using 5.2 as the cutoff for the presence of fatigue, we found the following frequencies of fatigue: $3.5 \%$ in healthy controls, $45 \%$ in $\mathrm{MS}, 31 \%$ in patients with previous ischemic stroke, and $36 \%$ in patients with sleep-wake disorders. When using a cutoff of 4 (as suggested by some authors ${ }^{17,21}$ ), we found fatigue in $18 \%$ of healthy controls, $69 \%$ of MS patients, $49 \%$ of stroke patients, and $62 \%$ of patients with sleep-wake disorders.

\section{MS Patients $(\mathrm{n}=188)$}

We observed a tendency towards higher FSS scores with increasing age (Pearson $\mathrm{r}=0.18, \mathrm{P}=0.01$ ). FSS scores were significantly correlated with physical disability (expanded disability status scale, EDSS) $(\mathrm{r}=0.34, \mathrm{P}<0.01)$, but not with disease duration $(\mathrm{r}=0.11, \mathrm{P}=0.13)$, gender $(\mathrm{P}=0.86)$, or educational status $(\mathrm{r}=-0.05, \mathrm{P}=0.50)$.

\section{Patients with Previous Ischemic Stroke $(n=235)$}

No correlation was found between FSS scores and age $(\mathrm{P}=$ $0.95)$, duration from disease onset $(P=0.91)$, gender $(P=0.82)$, or educational status $(\mathrm{P}=0.96)$.

\section{Patients with Sleep-Wake Disorders $(n=429)$}

FSS and ESS values for specific sleep-wake disorders are shown in Table 3. There are significant differences of mean FSS scores between sleep diagnoses $(\mathrm{P}=0.04)$; patients with insomnia and narcolepsy had the highest scores $(4.78 \pm 1.51$ and $4.75 \pm 1.47$, respectively), and patients with restless legs syndrome had the lowest FSS scores (4.02 \pm 1.75$)$. Overall, we observed a modest yet significant correlation between FSS and ESS $(r=0.34, \mathrm{P}<0.01)$. In the subgroup of patients with narcolepsy with cataplexy and sleep apnea we found the highest correlation $(\mathrm{r}=0.71$ and 0.48 , respectively, $\mathrm{P}<0.01)$, whereas the correlation was lowest for patients with insomnia $(\mathrm{r}=0.21, \mathrm{P}=0.11)$.

\section{Healthy Subjects $(n=454)$}

We found no significant associations of FSS scores with age or education. There was a weak association between FSS score and gender: the mean difference of FSS in females compared to males was $0.21(\mathrm{P}=0.04)$. Differences in bedtime of $\geq 2$ hours between weekday and weekend - suggestive of behaviorally induced insufficient sleep syndrome (BIISS) — had no influence on the FSS score (BIISS: $\mathrm{n}=97$, FSS $3.07 \pm 0.97$; non-BIISS: $\mathrm{n}=$ 357 , FSS $2.98 \pm 1.11 ; \mathrm{P}=0.3)$. ESS scores, however, differed between the 2 groups (BIISS: $6.79 \pm 3.53$, non-BIISS: $5.93 \pm 3.58$, $\mathrm{P}=0.04)$. In healthy subjects, there was a modest but significant correlation between FSS and ESS $(r=0.28, \mathrm{P}<0.01)$.

\section{Correlation Between FSS and VAS}

We observed a highly significant correlation $(\mathrm{r}=0.69, \mathrm{P}<$ 0.01 ) between FSS scores and fatigue as indicated on the VAS. This correlation was higher in patients (MS: $r=0.79$, ischemic stroke: $r=0.70$, sleep-wake disorders: $r=0.71)$ than in healthy subjects $(\mathrm{r}=0.52)$.

\section{Item Analysis, Internal Consistency, and Test-Retest Variability of the FSS}

Cronbach coefficient $\alpha$ for the entire sample was 0.93 , showing a high degree of internal consistency of the FSS (Table 4).

Table 3 - Correlation Between Epworth Sleepiness Scale (ESS) and Fatigue Severity Scale (FSS) Values in Different Sleep-Wake Disorders $($ Mean $\pm \mathrm{SD})$

\begin{tabular}{lcr} 
Sleep-wake disorder & n & \multicolumn{1}{c}{$\begin{array}{c}\text { ESS } \\
\text { mean } \pm \text { SD }\end{array}$} \\
Narcolepsy with cataplexy & 22 & $15.00 \pm 5.23$ \\
RLS & 79 & $8.59 \pm 5.54$ \\
Sleep apnea & 108 & $10.76 \pm 5.8$ \\
Insomnia & 62 & $7.39 \pm 5.18$ \\
Parasomnia & 25 & $7.32 \pm 3.96$ \\
EDS/hypersomnia of other origin & 84 & $10.47 \pm 4.95$ \\
Other sleep-wake disorders* & 49 & $8.85 \pm 4.79$ \\
& \\
RLS: restless legs syndrome, EDS: excessive daytime sleepiness. \\
*sleep related headache, nocturnal epilepsy, circadian rhythm disorders. \\
**Correlation between ESS and FSS.
\end{tabular}

\section{FSS}

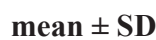

$4.75 \pm 1.47$

$4.02 \pm 1.75$

$4.11 \pm 1.64$

$4.78 \pm 1.51$

$4.64 \pm 1.45$

$4.55 \pm 1.70$

$4.18 \pm 1.56$

$\begin{array}{cc}\text { r } & \begin{array}{c}\text { Pearson** } \\ \text { P-value } \\ 0.71\end{array} \\ 0.28 & 0.01 \\ 0.48 & 0.01 \\ 0.21 & <0.01 \\ 0.31 & 0.11 \\ 0.33 & 0.14 \\ 0.45 & <0.01 \\ & <0.01\end{array}$


Table 4-Item Analysis of the FSS in Patients with MS, Ischemic Stroke, and Sleep-Wake Disorders, and in Healthy Controls

Item number mean \pm SD corrected item to Cronbach $\alpha$

a) multiple sclerosis $(\mathrm{n}=188)$

$\begin{array}{lll}1 & 5.28 \pm 1.58 & 0.48 \\ 2 & 4.93 \pm 1.91 & 0.72 \\ 3 & 4.69 \pm 2.02 & 0.82 \\ 4 & 5.04 \pm 1.91 & 0.82 \\ 5 & 4.45 \pm 1.98 & 0.85 \\ 6 & 4.85 \pm 2.06 & 0.85 \\ 7 & 3.99 \pm 2.05 & 0.77 \\ 8 & 4.52 \pm 2.20 & 0.79 \\ 9 & 4.19 \pm 2.21 & 0.78\end{array}$

Total $\quad 4.66 \pm 1.64$

b) ischemic stroke $(\mathrm{n}=235)$

$\begin{array}{lll}1 & 4.65 \pm 1.98 & 0.7 \\ 2 & 4.11 \pm 2.00 & 0.77 \\ 3 & 4.03 \pm 2.12 & 0.85 \\ 4 & 4.28 \pm 2.13 & 0.86 \\ 5 & 3.69 \pm 2.21 & 0.86 \\ 6 & 3.98 \pm 2.26 & 0.85 \\ 7 & 3.53 \pm 2.19 & 0.84 \\ 8 & 3.68 \pm 2.34 & 0.85 \\ 9 & 3.51 \pm 2.23 & 0.86 \\ \text { Total } & \mathbf{3 . 9 0} \pm \mathbf{1 . 8 5} & \end{array}$

0.70

0.77

0.85

0.86

0.86

0.85

0.84

0.85

0.86

) sleep-wake disorders $(n=429)$

\begin{tabular}{|c|c|}
\hline 1 & $5.07 \pm 1.80$ \\
\hline 2 & $4.23 \pm 1.90$ \\
\hline 3 & $4.28 \pm 1.99$ \\
\hline 4 & $4.61 \pm 1.92$ \\
\hline 5 & $4.41 \pm 2.10$ \\
\hline 6 & $4.06 \pm 2.05$ \\
\hline 7 & $3.90 \pm 2.06$ \\
\hline 8 & $4.44 \pm 2.21$ \\
\hline 9 & $4.16 \pm 2.22$ \\
\hline Total & $4.34 \pm 1.64$ \\
\hline
\end{tabular}

d) healthy controls $(n=454)$

$\begin{array}{llll}1 & 5.09 \pm 1.60 & 0.32 & 0.86 \\ 2 & 3.11 \pm 1.66 & 0.41 & 0.85 \\ 3 & 2.75 \pm 1.44 & 0.63 & 0.83 \\ 4 & 3.94 \pm 1.71 & 0.50 & 0.84 \\ 5 & 2.66 \pm 1.58 & 0.67 & 0.82 \\ 6 & 2.50 \pm 1.57 & 0.66 & 0.83 \\ 7 & 2.41 \pm 1.55 & 0.65 & 0.83 \\ 8 & 2.30 \pm 1.73 & 0.65 & 0.83 \\ 9 & 2.16 \pm 1.55 & 0.68 & 0.82 \\ \text { Total } & \mathbf{3 . 0 0} \pm \mathbf{1 . 0 8} & & \mathbf{0 . 8 5}\end{array}$

entire sample $(\mathrm{n}=1306)$

Total
0.60

0.60

0.79

0.83

0.83

0.77

0.80

0.79

0.81

0.85

0.93

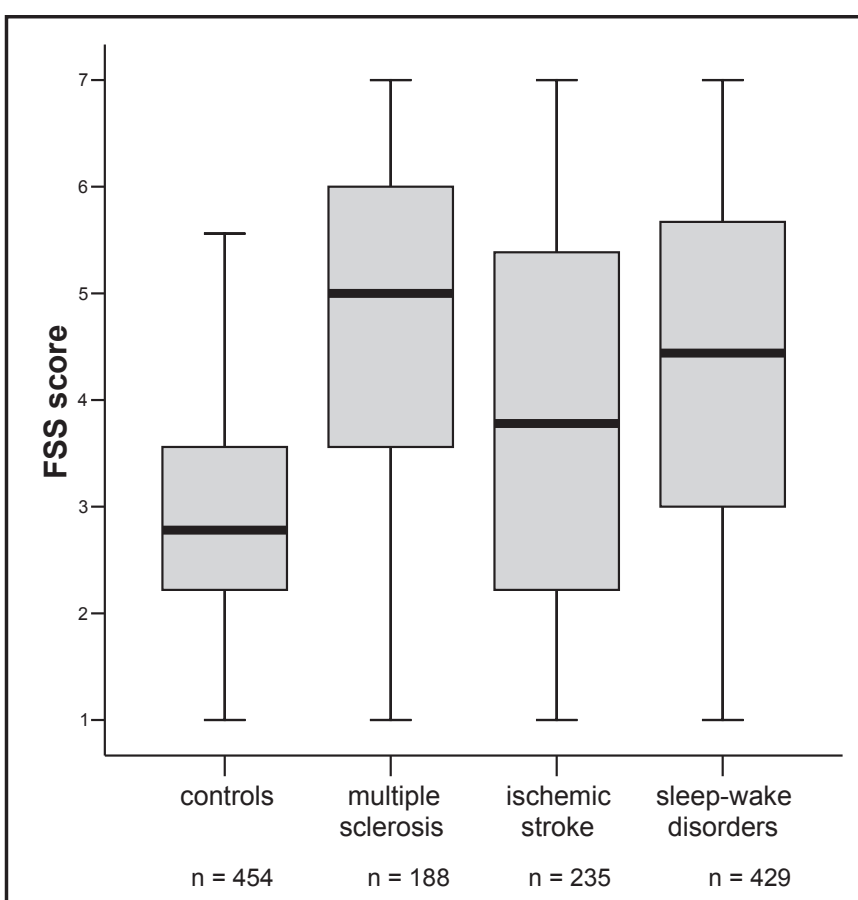

Figure 2-Boxplots showing the median and interquartile range of FSS scores in healthy controls and patients with MS, previous ischemic stroke, and sleep-wake disorders.

\section{DISCUSSION}

We show for the first time in a large sample size study that the FSS is a valuable tool to assess and quantify fatigue, as it differentiates between patients with various diseases and healthy subjects. Our data obtained in healthy subjects provide normal reference values with an upper limit of the normal range of 3.10. The FSS shows an excellent internal consistency, demonstrated by a Cronbach $\alpha$ coefficient of 0.93 , which is higher than in the earlier small sample size studies in MS patients. ${ }^{11,13,17}$ The reliability of the FSS is further reflected by the high testretest reliability. Based on our data, the minimal difference in FSS scores that can be reliably detected is 0.15 . In addition, a high degree of correlation of FSS scores and VAS results could be demonstrated.

We found that the FSS is useful to distinguish frequency and severity of fatigue between healthy subjects and patients with MS, different sleep-wake disorders, and ischemic stroke.

Based on our findings and on previous reports on the prevalence of fatigue, we suggest that a FSS score $\geq 4$ be interpreted as indicative of fatigue. ${ }^{2-5,11,13,17}$ When using 5.2 (mean $+2 \mathrm{SD}$ ) as the cutoff for the presence of fatigue, we obtained fatigue prevalences that were clearly lower than previously published prevalences of fatigue. In MS for instance, the prevalence of fatigue is well documented and ranges from $76 \%$ to $92 \%,{ }^{2-5}$ suggesting that a cutoff of 4 (obtained fatigue prevalence: $69 \%$ ) would be more appropriate than 5.2 (obtained fatigue prevalence: $45 \%$ ). Similarly, item 5 of the FSS ("Fatigue causes frequent problems for me") indicates the presence of fatigue in tested subjects. The score of this item was $\geq 5$ (affirmative answer) in $16 \%$ of healthy controls, $55 \%$ of MS patients, $36 \%$ of patients with previous ischemic stroke, and $51 \%$ of patients with sleep-wake disorders. This finding again is in favor of a 
cutoff of 4 for the upper limit of the normal range of the FSS.

The mean FSS scores for MS of this study were similar to those of the original validation study, a recent Turkish study, and other reports, ${ }^{11,13,17}$ indicating that the influences of language and cultural background are negligible in the FSS. Mean FSS scores for healthy controls $(3.00 \pm 1.08)$, however, were higher than in the study of Krupp et al. $(2.3 \pm 0.7)$. The main reason for this difference is probably our larger sample size of healthy subjects (454 vs. 20), which is probably more representative for the general population. Our results show that fatigue is frequent even in healthy controls, and that the prevalence of fatigue in the general population is largely independent of age, gender, and education. The reason for this finding remains unclear. Based on our questionnaires, chronic sleep deprivation (also referred to as behaviorally induced insufficiant sleep syndrome, BIISS, in the terminology of the American Academy of Sleep Medicine) did not account for fatigue in our large control group. In our study, however, BIISS was only assessed by questionnaires and not confirmed by actigraphy.

Fatigue was more pronounced in MS patients than patients with ischemic stroke and most patients with sleep-wake disorders. This underscores the high burden of fatigue in MS patients. ${ }^{18-20}$ In MS patients, severity of fatigue correlated with the degree of physical disability (EDSS score), but not with disease duration, age, or gender. Again, these results are in line with the findings of previous studies. ${ }^{18-20}$

Fatigue in patients with ischemic stroke was more pronounced than in healthy subjects, but less severe than in MS. Interestingly, we could not find a correlation between fatigue severity and the interval between stroke and study completion. This suggests that - in comparison to neurological symptoms, which may gradually improve after stroke-fatigue may persist as a stable complaint after stroke, at least during the first 2 years (observation time in this study).

Among patients with sleep-wake disorders, the highest FSS scores were found in patients with insomnia (4.78) and with narcolepsy (4.75), whereas lowest scores were found in RLS patients (4.02). Correlations between the ESS (assessment of subjective sleepiness) and the FSS were generally low and best in narcolepsy with cataplexy and sleep apnea. This confirms the concept that fatigue and excessive daytime sleepiness usually do not correspond but probably reflect different symptoms. This question, however, needs to be addressed with clinical sleep studies.

A limitation in our study was that the presence of depression was not assessed. In MS, several studies revealed a significant association between fatigue and depression, ${ }^{17,19,21}$ but in the validation study of Krupp et al., fatigue severity was largely independent of depressive symptoms. ${ }^{11}$ Similarly, a significant overlap between fatigue and depression exists in patients with recent ischemic stroke, but poststroke fatigue may also develop and persist in the absence of depression. ${ }^{8-10}$ The mutual interplay between fatigue, depression, and other disease-related factors remain unclear in most disorders and has to be elucidated in further studies.

In conclusion, the awareness and assessment of fatigue is essential for the management of affected patients. Currently, pharmacological therapy of fatigue is restricted to nonspecific wake-promoting agents (e.g., modafinil), amantadine, and vari- ous antidepressants. At the moment, however, fatigue remains an underrecognized problem, which may show negative interaction with performance levels and outcome measures (such as EDSS in MS patients, or Barthel Index in stroke patients for assessment of stroke-related disability), and therefore needs to be taken into consideration when caring for these patients. The scale allows detection and monitoring of disease-related fatigue and may indicate the need of appropriate interventions. In addition, reliable determination of fatigue severity and its variation during the time course of a disease are the prerequisite to develop future therapies to alleviate this troublesome symptom. The FSS constitutes a valid instrument to assess and quantify fatigue for such clinical and research purposes.

\section{ACKNOWLEDGMENTS}

We are indebted to Dr. D. Weniger, Prof. Dr. F. Gutzwiller, Dr. T. Tchelidze, Dr. L. Bachmann, I. Leuthold-Wyss und M. Leuthold for their valuable collaboration.

\section{DISCLOSURE STATEMENT}

This was not an industry supported study. Dr. Blochhas received research support from Respironics, ResMed, and Weinmann $\mathrm{AG}$ and has had the free use of monitoring equipment from VivoMetrics.

\section{REFERENCES}

1. Chaudhuri A, Behan PO. Fatigue in neurological disorders. Lancet 2004;363:978-88.

2. Krupp LB, Coyle PK, Doscher C, et al. Fatigue therapy in multiple sclerosis: results of a double-blind, randomized, parallel trial of amantadine, pemoline, and placebo. Neurology 1995;45:1956-61.

3. Branas P, Jordan R, Fry-Smith A, et al. Treatments for fatigue in multiple sclerosis: a rapid and systematic review. Health Technol Assess 2000;4:1-61.

4. Zifko UA. Management of fatigue in patients with multiple sclerosis. Drugs 2004;64:1295-1304.

5. Lichstein KL, Means MK, Noe SL, Aguillard RN. Fatigue and sleep disorders. Behav Res Ther 1997;35:733-40.

6. Hossain JL, Ahmad P, Reinish LW, Kayumov L, Hossain NK, Shapiro CM. Subjective fatigue and subjective sleepiness: two independent consequences of sleep disorders? J Sleep Res 2005;14:245-53.

7. Ingles JL, Eskes GA, Phillips SJ. Fatigue after stroke. Arch Phys Med Rehabil 1999;80:173-8.

8. Choi-Kwon S, Han SW, Kwon SU, et al. Poststroke fatigue: characteristics and related factors. Cerebrovasc Dis 2005;19:84-90.

9. Staub F, Bogousslavsky J. Fatigue after stroke: a major but neglected issue. Cerebrovasc Dis 2001;12:75-81.

10. Bogousslavsky J. William Feinberg lecture 2002: emotions, mood, and behaviour after stroke. Stroke 2003;34:1046-50.

11. Krupp LB, LaRocca NG, Muir-Nash J, Steinberg AD. The fatigue severity scale. Application to patients with multiple sclerosis and systemic lupus erythematosus. Arch Neurol 1989;46:1121-3.

12. Mead G, Lynch J, Greig C, Young A, Lewis S, Sharpe M. Evaluation of fatigue scales in stroke patients. Stroke 2007;38:2090-5.

13. Hjollund NH, Andersen JH, Bech P. Assesssment of fatigue in chronic disease: a bibliographic study of fatigue measurement scales. Health Qual Life Outcomes 2007;5:12.

14. Bloch KE, Schoch OD, Zhang JN, Russi EW. German version of the Epworth sleepiness scale. Respiration 1999;66:440-7. 
15. Lin L. A concordance correlation coefficient to evaluate reproducibility. Biometrics 45;1989:255-68.

16. Cronbach LJ. Coefficient alpha and the internal structure of test. Psychometrika 1951;16:297-334.

17. Armutlu K, Korkmaz NC, Keser I, et al. The validity and reliability of the Fatigue Severity Scale in Turkish multiple sclerosis patients. Int J Rehabil Res 2007;30:81-5.

18. Flachenecker P, Kümpfel T, Kallmann B, et al. Fatigue in multiple sclerosis: a comparison of different rating scales and correlation to clinical parameters. Mult Scler 2002;8:523-6.
19. Pittion-Vouyovitch S, Debouverie M, Guillemin F, Vandenberghe $\mathrm{N}$, Anxionnat R, Vespignani H. Fatigue in multiple sclerosis is related to disability, depression and quality of life. J Neurol Sci 2006;243:39-45.

20. Bergamaschi R, Romani A, Versino M, Poli R, Cosi V. Clinical aspects of fatigue in multiple sclerosis. Funct Neurol 1997; 12:247-51.

21. Kaynak H, Altintaş A, Kaynak D, et al. Fatigue and sleep disturbance in multiple sclerosis. Eur J Neurol 2006;13:1333-9. 


\title{
Fatigue and excessive daytime sleepiness in idiopathic Parkinson's disease differently correlate with motor symptoms, depression and dopaminergic treatment
}

\author{
P. O. Valko ${ }^{a}$, D. Waldvogel ${ }^{a}$, M. Weller ${ }^{a}$, C. L. Bassetti ${ }^{a, c}$, U. Held ${ }^{b}$ and C. R. Baumann ${ }^{a}$ \\ ${ }^{\mathrm{a}}$ Department of Neurology; and ${ }^{\mathrm{b}}$ Horton Centre for Patient-oriented Research, University Hospital of Zurich, Zurich: and ${ }^{\mathrm{c}}$ Neurocenter of \\ Southern Switzerland, Lugano, Switzerland
}

\section{Keywords:}

depression, disease duration, dopaminergic treatment, excessive daytime sleepiness, fatigue, idiopathic

Parkinson's disease

Received 7 November 2009 Accepted 10 March 2010

\begin{abstract}
Background and purpose: A comprehensive study of both fatigue and excessive daytime sleepiness (EDS) in association with Parkinson's disease (PD)-related symptoms and treatment has not been performed yet. To assess the frequency and severity of fatigue and EDS in patients with idiopathic PD and to study their relation to motor and non-motor symptoms and dopaminergic treatment.

Methods: We prospectively assessed Fatigue Severity Scale (FSS) scores, Epworth Sleepiness Scale (ESS) scores, Beck Depression Inventory (BDI) scores, severity (Unified PD Rating Scale, UPDRS, part III; Hoehn \& Yahr staging) and duration of the disease, and the current dopaminergic treatment in 88 consecutive patients with idiopathic PD.

Results: Fatigue was found in $52(59 \%)$, EDS in 42 (48\%), and both complaints in 31 $(35 \%)$ patients. Fatigued patients had higher UPDRS III scores $(23.5 \pm 11.1$ vs. $18.6 \pm 7.6, P=0.03)$, higher Hoehn \& Yahr staging $(2.4 \pm 0.9$ vs. $2.1 \pm 0.7$, $P=0.03)$, and higher BDI scores $(13.4 \pm 7.1$ vs. $9.1 \pm 5.8, P=0.004)$ than nonfatigued patients. In contrast, UPDRS III, Hoehn \& Yahr, and BDI scores did not differ between patients with or without EDS. However, the type of dopaminergic treatment (levodopa monotherapy versus combination of levodopa/dopamine agonists) was associated with significant differences in ESS ( $8.5 \pm 5.2$ vs. $10.8 \pm 4.3, P=0.04)$, but not FSS scores $(4.1 \pm 1.5$ vs. $4.3 \pm 1.5, P=0.55)$. Disease duration correlated with ESS scores $(r=0.32, P=0.003)$, but not with FSS scores $(r=-0.02, P=0.82)$.

Conclusions: In PD, there is a significant overlap of fatigue and EDS, but the two symptoms are differently correlated with the severity of motor symptoms, disease duration, depression, and dopaminergic treatment.
\end{abstract}

\section{Introduction}

Sleep-wake disturbances including fatigue and excessive daytime sleepiness (EDS) are important non-motor features of idiopathic Parkinson's disease (PD). EDS is present in up to $50-75 \%$ of patients with $\mathrm{PD}$, thereby significantly surpassing the frequency of EDS in other brain disorders, e.g. multiple sclerosis, ischemic stroke, traumatic brain injury [1-6]. Recently, the issues of EDS and 'sleep attacks' defined as episodes of EDStriggered short involuntary sleep at inappropriate situ-

Correspondence: P. O. Valko, Department of Neurology, University Hospital of Zurich, Frauenklinikstrasse 26, 8091 Zurich, Switzerland (tel.: +41 4425555 11; fax: + 414425543 80; e-mail: philipp. valko@usz.ch). ations, and their association with the use of dopaminergic agents, have received increasing attention [7-10]. Fatigue, on the other hand, often remains unrecognized, despite its high prevalence (40-60\%) [1,11-13]. Moreover, the association between fatigue, treatment and severity of motor symptoms and depression in PD is still under debate [14-18]. Alves et al. [19] found a significant correlation between fatigue and EDS in PD. The etiology of fatigue and EDS in PD has remained controversial. Some authors attributed impaired vigilance in PD to the high prevalence of sleep abnormalities, including insomnia, sleep apnea, fragmented sleep architecture, and reduced slow wave and REM sleep [20-23].

So far, however, there is no comprehensive study that systematically and prospectively assessed both fatigue 
and EDS in PD, and their relation to PD symptoms. We therefore aimed at characterizing fatigue and EDS in $\mathrm{PD}$, ascertaining the extent of overlap between the two symptoms, and associating them to other motor and non-motor symptoms, and to dopaminergic medication.

\section{Methods}

This prospective study was conducted at the Department of Neurology of the University Hospital of Zurich, Switzerland, between August 2007 and May 2008. The study protocol was approved by the Ethics Committee of the Canton of Zurich, specialized subcommittee for Psychiatry, Neurology, Neurosurgery.

\section{Subjects}

We included 88 consecutive patients with PD from our Movement Disorders outpatient clinic. The diagnosis of PD was made according to international diagnostic criteria $[24,25]$. Patients with other extrapyramidal or neurodegenerative disorders were excluded.

\section{Clinical assessments}

We rated motor symptoms according to the motor subset of the Unified Parkinson's Disease Rating Scale (UPDRS III) in off condition [26]. PD severity was determined by Hoehn \& Yahr staging [27]. A detailed, structured interview and clinical examination allowed assessing the presence of the following motor and non-motor symptoms: rapid eye movement (REM) sleep behavior disorder (RBD), insomnia defined as deterioration of nighttime sleep compared to prior disease onset, hallucinations, autonomic disturbances including orthostatic hypotension, constipation, impotence, paroxysmal sweating, and inadequate bladder emptying. Patients were considered to suffer from PD-related insomnia, if they judged their ability to fall asleep and to sleep through as significantly worse than before disease onset. Information on predominantly affected body side at disease onset, and type of PD (tremor-dominant versus akinetic-rigid) was also obtained. Overnight video-polysomnography was performed in 26 patients $(30 \%)$. Video-polysomnography was performed in patients with suspected RBD.

\section{Questionnaire for the assessment of fatigue, EDS, and mean bedtimes}

The Fatigue Severity Scale (FSS) was used to assess the presence and severity of fatigue. The FSS represents the most commonly used self-report questionnaire to measure fatigue and has recently been validated for a variety of neurological disorders in a large sample size study $(n=1304)$, showing an excellent internal consistency and reliability [28]. Several studies have used the FSS in patients with PD, although the scale has not yet been validated for PD [29]. We performed a reliability statistic in this study sample, finding a high internal consistency (Cronbach's $\alpha=0.94$ ) and good item consistencies. For the assessment of EDS, we used the validated Epworth Sleepiness Scale (ESS), where a score $\geq 10$ indicates EDS [30]. The questionnaire also included questions on mean time in bed per $24 \mathrm{~h}$. The term 'increased sleep duration' was used in patients with PD with mean times in bed $\geq 10 \mathrm{~h}$ per day. Furthermore, the Beck Depression Inventory (BDI) was used for evaluation of depression with a cut-off of $\geq 12$ for the presence of depression [31].

\section{Dopaminergic medication}

To compare different dopaminergic medications at doses of equivalent efficacy, we converted all dosages to levodopa dosage equivalents (LDE) according to the following formula $[10,32]$ : $\mathrm{LDE}=$ (regular levodopa dose $\times 1)+($ levodopa controlled release dose $\times$ $0.75)+($ pramipexole dose $\times 67)+($ ropinirole dose $\times 16.67)+($ pergolide dose $\times 100)+($ cabergoline dose $\times 50)+($ levodopa/entacapone dose $\times 1.25)$.

\section{Data analysis and statistics}

Data analyses include a synopsis of the demographic and clinical characteristics of our study population, the calculation of the prevalences of the different vigilance disturbances as well as their interactions, and their association with other PD-related motor and nonmotor symptoms. For this purpose, we used spss (version 16.0; Pearson Education Deutschland $\mathrm{GmbH}$, München, Germany), stata version 9.2 (Stata Corp LP, College Station, TX, USA), and R version 2.10 (R Foundation for Statistical Computing, Vienna, Austria, http://www.R-project.org). for statistical analyses. Group data are described by means and standard deviations (SD). For univariate analysis, we used Student's $t$-test for numerical scale variables and $\chi^{2}$-test for nominal scale variables. Correlation analyses were performed using the Pearson's coefficient. We used multinomial regression to assess the influence of depression (BDI) and motor symptoms (UPDRS III) on the odds ratio (OR) of only EDS (without fatigue), only fatigue (without EDS), and both symptoms. The reference category in both regression models was the group of patients with no EDS and no fatigue. Significance was accepted at $P<0.05$. Internal consistency 
Table 1 Demographic and clinical characteristics of 88 patients with idiopathic Parkinson's disease. To describe dopaminergic treatment, the numbers and percentages of patients with a respective dopaminergic treatment are provided

\begin{tabular}{|c|c|}
\hline \multicolumn{2}{|l|}{ Demographic data } \\
\hline Age (years) & $67.5 \pm 9.7(38-84)$ \\
\hline Gender, male & $61(69 \%)$ \\
\hline \multicolumn{2}{|l|}{ Parkinson-related data } \\
\hline Disease duration (years) & $9.8 \pm 5.4(2-28)$ \\
\hline UPDRS III & $21.5 \pm 10.1(4-59)$ \\
\hline Hoehn \& Yahr & $2.3 \pm 0.8(1-5)$ \\
\hline Affected side, left & $50(57 \%)$ \\
\hline Type, tremor-dominant & $23(26 \%)$ \\
\hline Type, akinetic-rigid & $51(58 \%)$ \\
\hline Autonomic disturbances & $31(35 \%)$ \\
\hline \multicolumn{2}{|l|}{ Neuropsychiatric symptoms } \\
\hline Hallucinations & $21(24 \%)$ \\
\hline History of REM sleep behavior disorder & $24(27 \%)$ \\
\hline BDI & $11.6 \pm 6.9(0-35)$ \\
\hline Depression (BDI $\geq 12$ ) & $37(42 \%)$ \\
\hline \multicolumn{2}{|l|}{ Sleep-wake disturbances } \\
\hline FSS & $4.2 \pm 1.6(1.1-7)$ \\
\hline Fatigue (FSS $\geq 4.0$ ) & $52(59 \%)$ \\
\hline ESS & $9.7 \pm 5.0(2-24)$ \\
\hline EDS $(\mathrm{ESS} \geq 10)$ & $42(48 \%)$ \\
\hline Time in bed (h) & $8.4 \pm 1.5(4-13.5)$ \\
\hline Increased sleep duration (bedtime $\geq 10 \mathrm{~h}$ ) & $15(17 \%)$ \\
\hline Insomnia & $59(67 \%)$ \\
\hline \multicolumn{2}{|l|}{ Dopaminergic treatment } \\
\hline Levodopa & $46(52 \%)$ \\
\hline Only levodopa & $33(38 \%)$ \\
\hline DA & $53(60 \%)$ \\
\hline Non-ergot DA & $46(52 \%)$ \\
\hline Ergot DA & $8(9 \%)$ \\
\hline Only DA & $9(10 \%)$ \\
\hline Both levodopa and DA & $44(50 \%)$ \\
\hline Levodopa + entacapone & $32(36 \%)$ \\
\hline No dopaminergic drugs & $2(2 \%)$ \\
\hline LDE (mg/day) & $681 \pm 402(0-1581)$ \\
\hline
\end{tabular}

UPDRS, Unified Parkinson's Disease Rating Scale (part III = motor score); BDI, Beck Depression Inventory; REM sleep, rapid eye movement sleep; FSS, Fatigue Severity Scale; ESS, Epworth Sleepiness Scale; DA, dopamine agonist; LDE, levodopa dosage equivalent.

of the FSS in this study sample was assessed by calculating the item to total correlation and the Cronbach's $\alpha$ statistics [33].

\section{Results}

\section{Subjects and treatment}

The demographic and clinical characteristics of our 88 patients are shown in Table 1. The majority of patients received a combination of levodopa with a dopamine agonist (DA; 44 patients, 50\%), whereas 33 patients $(38 \%)$ had levodopa monotherapy and nine patients $(10 \%)$ had DA monotherapy. No patients were on rasagiline or selegiline treatment.
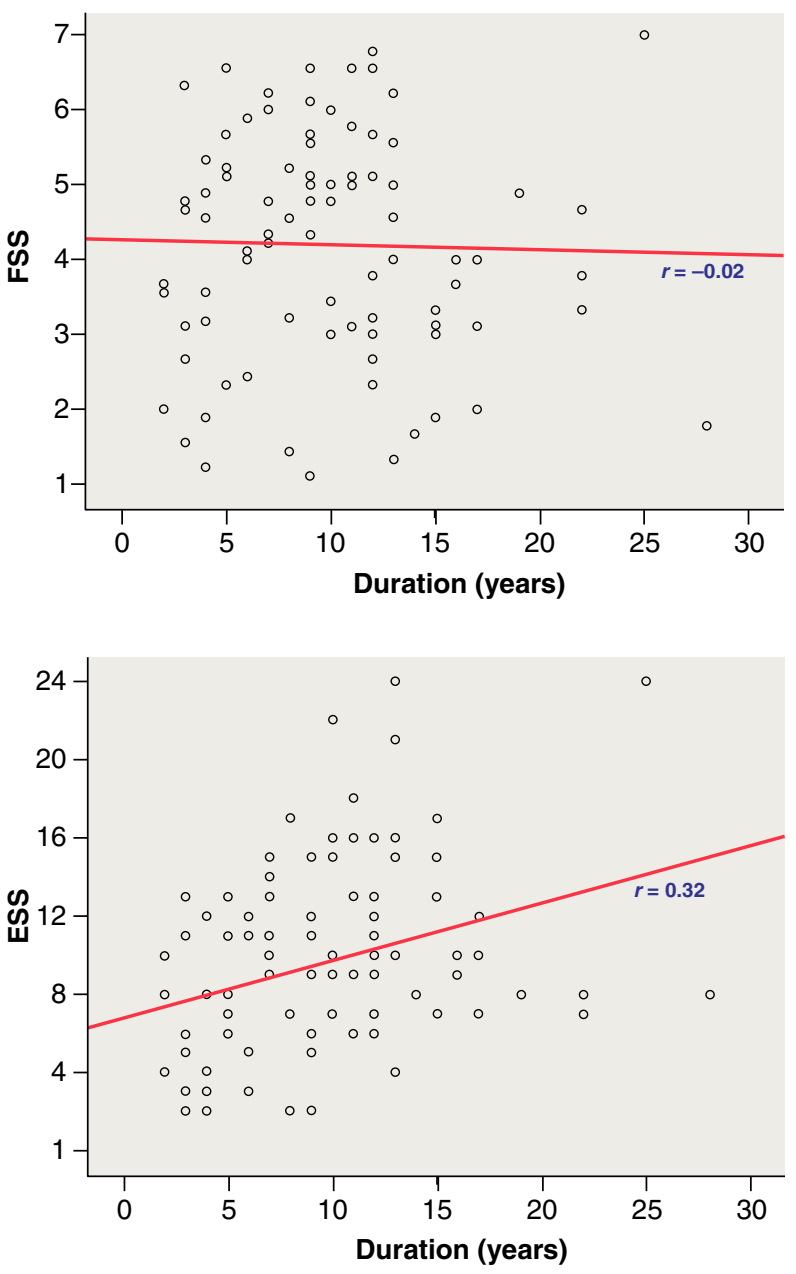

Figure 1 Association of fatigue and excessive daytime sleepiness (EDS) with disease duration. Individual scores for Fatigue Severity Scale (FSS) or Epworth Sleepiness Scale (ESS) were plotted against disease duration.

\section{Prevalence of fatigue, EDS, and increased sleep duration}

Fatigue (FSS $\geq 4.0$ ) was present in 52 patients $(59 \%)$; mean FSS for all patients was $4.2 \pm 1.6$. EDS (ESS $\geq 10$ ) was reported by 42 patients $(48 \%)$; mean ESS was $9.7 \pm 5.0$. Thirty-one patients $(35 \%)$ suffered from both fatigue and EDS, whereas 11 patients (13\%) had only EDS and 21 patients $(24 \%)$ had only fatigue. Twenty-five patients (28\%) indicated neither fatigue nor EDS. Increased sleep duration was found in 15 patients (17\%); mean time in bed was $8.4 \pm 1.5 \mathrm{~h}$. The distribution of these sleep-wake disturbances was independent of age and gender. However, the prevalence and severity of EDS but not of fatigue or increased sleep duration became higher with longer disease duration. Disease duration correlated with ESS scores $(r=0.32, P=0.003)$, but not with FSS scores 
Table 2 Comparison of patients with Parkinson's disease with and without fatigue (FSS $\geq 4.0$ ), and with and without EDS (ESS $\geq 10$ )

\begin{tabular}{|c|c|c|c|c|c|c|}
\hline & Fatigued patients & Non-fatigued patients & $P$ & Patients with EDS & Patients without EDS & $P$ \\
\hline$n$ & $52(59 \%)$ & $36(41 \%)$ & & $42(48 \%)$ & $46(52 \%)$ & \\
\hline Age & $67.2 \pm 10.5$ & $67.8 \pm 8.6$ & 0.77 & $68.2 \pm 9.2$ & $66.8 \pm 10.2$ & 0.52 \\
\hline Gender $(\mathrm{m} / \mathrm{f})$ & $39 / 13$ & $22 / 14$ & 0.13 & $31 / 11$ & $30 / 16$ & 0.26 \\
\hline Disease duration & $9.4 \pm 4.6$ & $10.3 \pm 6.5$ & 0.47 & $10.3 \pm 4.5$ & $9.4 \pm 6.1$ & 0.44 \\
\hline UPDRS III & $23.5 \pm 11.1$ & $18.6 \pm 7.6$ & 0.03 & $22.3 \pm 10.5$ & $20.8 \pm 9.8$ & 0.50 \\
\hline Hoehn \& Yahr & $2.4 \pm 0.9$ & $2.1 \pm 0.7$ & 0.03 & $2.3 \pm 0.8$ & $2.3 \pm 0.8$ & 0.91 \\
\hline Autonomic disturbances & $24 / 52(46 \%)$ & $7 / 36(19 \%)$ & 0.008 & $16 / 42(38 \%)$ & $15 / 46(33 \%)$ & 0.38 \\
\hline ESS & $11.1 \pm 5.1$ & $7.6 \pm 4.2$ & 0.001 & & & \\
\hline EDS $(E S S \geq 10)$ & $31 / 52(60 \%)$ & $11 / 36(31 \%)$ & 0.007 & & & \\
\hline FSS & & & & $4.8 \pm 1.4$ & $3.7 \pm 1.5$ & $<0.001$ \\
\hline Fatigue (FSS $\geq 4.0$ ) & & & & $31 / 42(74 \%)$ & $21 / 46(46 \%)$ & 0.007 \\
\hline Time in bed $(\mathrm{h})$ & $8.7 \pm 1.7$ & $8.1 \pm 1.2$ & 0.12 & $8.5 \pm 1.7$ & $8.4 \pm 1.4$ & 0.90 \\
\hline Insomnia & $40 / 52(77 \%)$ & $19 / 36(53 \%)$ & 0.02 & $33 / 42(79 \%)$ & $26 / 46(57 \%)$ & 0.04 \\
\hline BDI & $13.4 \pm 7.1$ & $9.1 \pm 5.8$ & 0.004 & $10.9 \pm 5.5$ & $12.3 \pm 8.0$ & 0.36 \\
\hline Depression (BDI $\geq 12$ ) & $27 / 52(52 \%)$ & $10 / 36(28 \%)$ & 0.02 & $16 / 42(38 \%)$ & $21 / 46(46 \%)$ & 0.31 \\
\hline LDE (mg/day) & $732 \pm 417$ & $609 \pm 375$ & 0.16 & $729 \pm 372$ & $638 \pm 427$ & 0.29 \\
\hline \multicolumn{7}{|l|}{ PSG } \\
\hline Latency NREM2 (min) & $21.7 \pm 30.2$ & $41.3 \pm 38.4$ & 0.17 & $33.2 \pm 44.4$ & $25.6 \pm 20.0$ & 0.59 \\
\hline Latency REM (min) & $101.4 \pm 43.2$ & $92.5 \pm 31.0$ & 0.58 & $83.4 \pm 25.7$ & $113.5 \pm 44.4$ & 0.05 \\
\hline Arousal index & $11.9 \pm 9.0$ & $24.0 \pm 30.2$ & 0.16 & $10.0 \pm 5.4$ & $24.1 \pm 28.0$ & 0.09 \\
\hline Sleep efficiency $(\%)$ & $68.8 \pm 21.5$ & $81.3 \pm 17.4$ & 0.14 & $71.8 \pm 23.9$ & $75.7 \pm 16.7$ & 0.64 \\
\hline PLMS index & $6.0 \pm 21.2$ & $36.6 \pm 49.1$ & 0.04 & $20.9 \pm 44.9$ & $14.2 \pm 26.4$ & 0.65 \\
\hline Wake (\%) & $31.2 \pm 21.5$ & $18.7 \pm 17.4$ & 0.14 & $28.2 \pm 23.9$ & $24.3 \pm 16.7$ & 0.64 \\
\hline
\end{tabular}

Bold values indicate significance.

UPDRS, Unified Parkinson's Disease Rating Scale (part III = motor score); BDI, Beck Depression Inventory; REM sleep, rapid eye movement sleep; FSS, Fatigue Severity Scale; ESS, Epworth Sleepiness Scale; EDS, excessive daytime sleepiness; LDE, levodopa dosage equivalent; PSG, polysomnography; NREM, non-rapid eye movement sleep; PLMS, periodic leg movements in sleep.

$(r=-0.02, P=0.82)$ or mean time in bed $(r=-0.17$, $P=0.12$ ) (Fig. 1).

\section{Polysomnographic data $(n=26)$}

Patients with a high arousal index, i.e. with frequent electroencephalographic arousals at night, had lower FSS scores $(r=-0.48, P=0.03)$. Similarly, a higher periodic leg movements in sleep (PLMS) index was found in non-fatigued than in fatigued patients $(37 \pm 49$ vs. $6 \pm 21, P=0.04)$. Fourteen patients $(54 \%)$ were diagnosed with moderate (apnea hypopnea index, AHI $=20-30 / \mathrm{h})$ to severe $(\mathrm{AHI} \geq 30 / \mathrm{h}$ ) sleep apnea; no correlation was found between AHI and FSS $(r=0.07, P=0.74)$ or ESS scores $(r=0.23, P=$ $0.27)$. Other associations between polysomnographic findings and the FSS, ESS, or mean bedtimes were not found (data not shown).

\section{The relation of fatigue to other PD-related symptoms}

Fatigued patients with PD had more severe motor symptoms than patients with $\mathrm{PD}$ without fatigue (UPDRS III: $23.5 \pm 11.1$ vs. $18.6 \pm 7.6, P=0.03$, Hoehn \& Yahr: $2.4 \pm 0.9$ vs. $2.1 \pm 0.7, P=0.03$ ) (Table 2). Our multinomial regression model confirmed this result: For motor symptoms (UPDRS III), we found a positive association for the comparison of fatigue alone versus the reference category: patients with PD with a 10 point higher value on the UPDRS scale have an estimated OR of $2.69(P=0.005)$ to suffer from fatigue.

Fatigued patients with $\mathrm{PD}$ more often reported PD-related insomnia (77\% vs. $53 \%$ respectively, $P=0.02)$ and autonomic disturbances (46\% vs. $19 \%$, $P=0.008)$. On the other hand, the presence of fatigue was not associated with PD features such as predominantly affected body side, disease type (tremor-dominant versus akinetic-rigid), or hallucinations. Fatigued patients with PD more often were depressed (52\% vs. $28 \%, \quad P=0.02)$ and had higher BDI scores $(13.4 \pm 7.1$ vs. $9.1 \pm 5.8, P=0.004)$.

\section{The relation of EDS to other PD-related symptoms and treatment}

Comparing patients with PD with and without EDS, we found no differences concerning severity of motor symptoms, confirmed in the multinomial regression model, predominantly affected body side, disease type (tremor-dominant versus akinetic-rigid), hallucinations, autonomic disturbances, or frequency, and severity of 
depression (Table 2). Insomnia was more prevalent in patients with PD with $\operatorname{EDS}(79 \%$ vs. $57 \%, P=0.04)$.

\section{The relation of increased sleep duration to other PD- related symptoms}

Patients with PD with increased sleep duration $(n=15)$ were more severely affected by motor symptoms (UPDRS III: $27.3 \pm 9.4$ vs. $20.3 \pm 9.9, P=0.02$ ) compared to patients with PD with mean time in bed $<10 \mathrm{~h}(n=73)$. There were no associations of the presence or severity of increased sleep duration with other PD symptoms such as autonomic disturbances, disease type, and predominantly affected body side.

\section{The relations between fatigue, EDS, and increased sleep duration in PD}

FSS scores significantly correlated with ESS scores $(r=0.48, P<0.001)$. EDS was almost twice as frequent amongst fatigued patients compared to nonfatigued patients ( $60 \%$ vs. $31 \%, P=0.007)$. Similarly, patients with PD with EDS had significantly higher FSS scores $(4.8 \pm 1.4$ vs. $3.7 \pm 1.5, P<0.001)$. Patients with PD with increased sleep duration $(n=15)$ were more fatigued (FSS: $5.1 \pm 1.2$ vs. $4.0 \pm 1.6, P=0.02$; prevalence of FSS $\geq 4.0: 87 \%$ vs. $53 \%, P=0.02$ ). On the other hand, there were no associations between increased sleep duration and EDS.

\section{The relation of fatigue, EDS, and increased sleep duration to dopaminergic treatment}

Presence and severity of fatigue were independent of LDE or daily doses of levodopa or ergot DA. However, patients with non-ergot DA had higher FSS scores $(4.5 \pm 1.5)$ than patients with PD that were not treated with non-ergot DA $(3.9 \pm 1.5, P=0.049)$. The presence of EDS and increased sleep duration was independent of whether patients were treated with levodopa or ergot DA. However, total LDE correlated mildly with the severity of EDS as assessed with the ESS $(r=0.22, P=0.04)$. Again, patients with non-ergot DA had higher ESS scores $(10.9 \pm 5.1)$ than patients without non-ergot DA $(8.4 \pm 4.7, P=0.02)$.

A comparison of patients under levodopa monotherapy $(n=33)$ and patients receiving both levodopa/ DA $(n=44)$ revealed no differences for FSS score and mean time in bed, but significantly higher ESS scores in patients taking both levodopa/DA $(10.9 \pm 4.3$ vs. $8.4 \pm 5.0, P=0.02$ ) (Table 3). Patients with levodopa monotherapy slept almost $1 \mathrm{~h}$ more per night than patients with both levodopa/DA $(8.9 \pm 1.2$ vs. $8.0 \pm 1.6, P=0.006$ ), and suffered less frequently
Table 3 Dopaminergic treatment: patients with only levodopa versus patients with both levodopa and DA

\begin{tabular}{|c|c|c|c|}
\hline & $\begin{array}{l}\text { Only } \\
\text { levodopa }\end{array}$ & $\begin{array}{l}\text { Both } \\
\text { levodopa + DA }\end{array}$ & $P$ \\
\hline$n$ & 33 & 44 & \\
\hline Sex (male/female) & $21 / 12$ & $34 / 10$ & 0.19 \\
\hline Age & $70.4 \pm 10.0$ & $66.5 \pm 9.1$ & 0.08 \\
\hline Disease duration & $8.9 \pm 5.3$ & $11.0 \pm 5.0$ & 0.08 \\
\hline UPDRS III & $22.6 \pm 9.9$ & $20.9 \pm 8.9$ & 0.43 \\
\hline Hoehn \& Yahr & $2.3 \pm 0.8$ & $2.4 \pm 0.8$ & 0.86 \\
\hline FSS & $4.0 \pm 1.5$ & $4.3 \pm 1.6$ & 0.43 \\
\hline ESS & $8.4 \pm 5.0$ & $10.9 \pm 4.3$ & 0.02 \\
\hline Time in bed & $8.9 \pm 1.2$ & $8.0 \pm 1.6$ & 0.01 \\
\hline Insomnia & $17 / 33(52 \%)$ & $35 / 44(80 \%)$ & 0.01 \\
\hline BDI & $13.6 \pm 8.2$ & $11.1 \pm 6.0$ & 0.13 \\
\hline \multicolumn{4}{|l|}{ PSG } \\
\hline Latency NREM2 (min) & $40.9 \pm 37.6$ & $24.1 \pm 35.4$ & 0.32 \\
\hline Latency REM (min) & $100.0 \pm 42.0$ & $97.7 \pm 39.1$ & 0.90 \\
\hline Arousal index & $9.3 \pm 5.2$ & $19.4 \pm 25.7$ & 0.32 \\
\hline Sleep efficiency $(\%)$ & $74.5 \pm 27.3$ & $72.3 \pm 19.0$ & 0.82 \\
\hline PLMS index & $30.4 \pm 39.5$ & $12.7 \pm 38.3$ & 0.32 \\
\hline Wake (\%) & $25.5 \pm 27.3$ & $27.8 \pm 19.0$ & 0.82 \\
\hline
\end{tabular}

Bold values indicate significance.

UPDRS, Unified Parkinson's Disease Rating Scale (part III = motor score); FSS, Fatigue Severity Scale; ESS, Epworth Sleepiness Scale; BDI, Beck Depression Inventory; DA, dopamine agonist; LDE, levodopa dosage equivalent; PSG, polysomnography; NREM, nonrapid eye movement sleep; PLMS, periodic leg movements in sleep.

from insomnia ( $52 \%$ vs. $80 \%, P=0.01)$. There was a trend toward longer disease duration in the patient group with both levodopa/DA $(8.9 \pm 5.3$ vs. $11.0 \pm 5.0, P=0.08)$. Analyzing the dose-dependent effect (LDE) of specific dopaminergic drugs on FSS/ ESS scores, we found no correlation for levodopa (FSS: $r=0.10, P=0.50$; ESS: $r=0.16, P=0.37$ ), ergot DA (FSS: $r=0.11, \quad P=0.80 ; \quad$ ESS: $r=-0.28$, $P=0.50$ ), and non-ergot DA (FSS: $r=0.24$, $P=0.11 ; \quad$ ESS: $r=0.26, \quad P=0.08$ ). For levodopa + entacapone, however, we found a significant dose-dependent increase of the FSS score $(r=0.51$, $P=0.003)$, but not of the ESS score $(r=0.16$, $P=0.37)$.

\section{Depression and vigilance impairment}

Thirty-seven patients with PD (42\%) fulfilled the criteria for depression (BDI $\geq 12$ ). BDI scores were not associated with motor symptoms as assessed by UPDRS III. Mean FSS was significantly higher in depressed $(4.8 \pm 1.2)$ than in non-depressed patients with PD $(3.8 \pm 1.7, P=0.004)$, and BDI scores correlated with FSS scores $(r=0.33, P=0.002)$. Similarly, in the multinomial regression model, only fatigue alone was associated with a significantly increased OR of depression (OR $=7.92,95 \% \mathrm{CI}(2.12$; 29.67), $P=0.002)$, whereas no significant effects of 


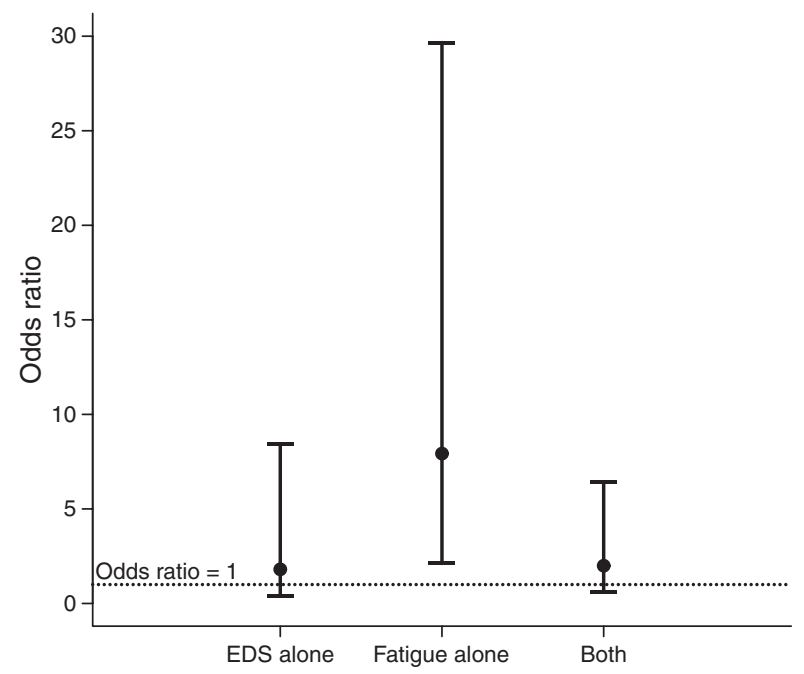

Figure 2 Multinomial regression model for depression against excessive daytime sleepiness (EDS) alone, fatigue alone or both, showing the respective odds ratios and corresponding 95\% confidence intervals $(\mathrm{CI})$.

depression were found for the contrasts of EDS alone or both fatigue and EDS (Fig. 2). Twenty-seven patients $(31 \%)$ suffered from both fatigue and depression, but the frequency of fatigue without concomitant depression was similarly high (25 patients, 28\%). Similarly, depressed patients had longer mean time in bed than non-depressed patients $(8.8 \pm 1.4 \mathrm{~h}$ vs. $8.2 \pm 1.6 \mathrm{~h}, P=0.04)$. On the other hand, there was no association between ESS and BDI.

\section{Discussion}

This study confirms that fatigue (59\%) and EDS (48\%) are frequent complaints in idiopathic PD. The indicated frequencies are comparable to other reports on fatigue (reported prevalences: 40-60\% [1,11-13]) and EDS (reported prevalences: $33-75 \%$ [10,34-37]) in PD. Although we found a significant overlap between fatigue and $\operatorname{EDS}(35 \%)$, the two complaints appeared to be differently associated with other clinical features of PD and with the dopaminergic medication.

Fatigue, but not EDS, correlated with the severity of motor symptoms (UPDRS III) and disease disability (Hoehn \& Yahr). In multiple sclerosis and ischemic stroke, a similar correlation between fatigue and physical disability as measured by the expanded disability status scale (EDSS), and by the National Institutes of Health Stroke Scale, NIHSS, respectively, has been found by various groups $[4,28,38-40]$. In PD, however, Lou et al. [15] failed to establish a relationship between motor disability and the presence of fatigue. In this particular study, however, the sample size was low $(n=39)$, fatigue was assessed with another questionnaire (Multidimensional Fatigue Inventory), and disease severity was estimated only by the Hoehn \& Yahr staging. Karlsen et al. [13] found an association between fatigue and disease severity that was not confirmed by multiple regression analysis. Conversely, and in agreement with our own findings, Alves et al. [19] showed in a prospective longitudinal study that fatigue correlates with PD severity.

Longer disease duration was associated with higher ESS but not higher FSS scores. A weak yet significant correlation between daytime sleepiness and longer disease duration was reported also by other groups $[10,36,41,42]$. On the other hand, little is known about the natural course of fatigue during progression of PD. Friedman et al. [1] found that the prevalence of fatigue remained stable over 9 years, although fatigue severity worsened in affected patients. Conversely, Alves et al. [19] prospectively assessed the prevalence of fatigue every 4 years and found an increase from $36 \%$ to $43 \%$ and then to $56 \%$. In this study, however, fatigue was assessed with different measurement tools at different time points, using the FSS only during the last assessment. The lack of correlation between fatigue and disease duration in our study might be explained by the high prevalence of fatigue already during the first years after disease onset, whereas the prevalence of EDS increased only later. In summary, the finding of a different evolution of fatigue and EDS during PD progression corroborates the concept of two distinct symptoms with different pathomechanisms. We suppose that EDS in PD is directly influenced by the underlying neuropathology, which includes a disruption of wake-promoting dopaminergic and hypocretinergic structures in the upper brainstem and the diencephalon [43-45]. It is conceivable that the increase in EDS with longer disease duration reflects the progression of neuropathological processes that supposedly start in the caudal brainstem and thereafter affect other areas in the upper brainstem, diencephalon, and cortex in an ascending pattern [43].

It may appear confusing that the UPDRS III scores were not different between patients with PD with and without EDS, considering that both EDS and motor disability significantly worsen with disease duration. We believe that the lack of such a difference could be because of distinct mechanisms, by which the neurodegenerative progression leads to a deterioration of motor symptoms on one hand and to an increase of sleepiness on the other hand. The neuroanatomical structures, which are involved in mediating the worsening of the two symptoms, are different and may be affected differently in an individual patient. Sleepiness is 
not directly caused by motor disability - as we suspect it is in part the case with fatigue - but rather by a disruption of wake-promoting brainstem structures.

Fatigue, on the other hand, is characterized by its multidimensionality encompassing cognitive, physical and psychosocial aspects, and seems to be influenced by a multitude of factors including motor symptoms, depression, and EDS. This diffuse and complex overlap with many other disease features probably explains, why fatigue - conversely to the more specific symptom of EDS - is present in many patients at an early disease stage. Multiple sclerosis is another disorder, in which a high prevalence of fatigue already at disease onset and a lack of correlation with disease duration were reported [28].

The presence of depression was significantly associated with fatigue, but no relationship between depression and EDS was established. Using a regression model, fatigue appeared to be correlated with depression only in the patient group with fatigue but without $\operatorname{EDS}(\mathrm{OR}=7.92, P=0.002)$. The overall prevalence of depression $(42 \%)$ in our study sample is comparable to other reports [46,47]. However, depression does not primarily account for the high frequency of fatigue in PD, as fatigue was similarly prevalent also in the non-depressed patients with $\mathrm{PD}$ of our study sample. The regression model found no correlation for depression and fatigue in the patient group suffering from both fatigue and EDS. This finding might be explained by different degrees of comorbidity between depression, fatigue, and EDS: In patients with both fatigue and EDS, fatigue could to some extent be primarily related to EDS, whereas fatigue in the absence of EDS is more probably to be related to depression. The complex interaction and overlaps of fatigue with other PD symptoms may reflect the sparse knowledge of the pathophysiology of fatigue, but also the low specificity in the assessment of this ill-defined symptom.

There is increasing literature dealing with the relationship between dopaminergic drugs and EDS [7-10,34], but the effects on fatigue have been largely neglected. Our data suggest that dopaminergic treatment exerts a stronger effect on EDS than on fatigue. We found a weak yet significant correlation between the levodopa equivalent daily dose and ESS scores $(r=0.22, P=0.04)$, which is in line with the finding of the Canadian Movement Disorder group in 638 patients with $\mathrm{PD}$ with various dopaminergic drugs $(r=0.16, P<0.001)$ [10]. In contrast, we found no significant correlation between levodopa equivalent daily dose and FSS scores. Comparing patients with levodopa monotherapy with patients under combination of levodopa with a DA, namely pramipexole or ropinirole, the two groups differed significantly for ESS, but not FSS scores. Similarly, Paus et al. [34] reported a higher risk of sleep attacks in patients with PD with a combination therapy of levodopa/DA compared to patients with levodopa monotherapy. In this study, however, ESS scores were not different between patients on levodopa monotherapy compared to levodopa/DA. As our two groups differed significantly concerning LDE, it remains unclear whether the differences in ESS scores are caused by the different dopaminergic treatment, particularly the use of DA in the second group, or simply by the higher LDE. Furthermore, the disease duration was longer in the patient group with both levodopa/DA, which may also account for the differences of ESS scores. This difference might have achieved statistical significance if the included patient number had been higher.

We did not assess detailed PD-related nighttime disturbances by means of a validated tool such as the Pittsburgh sleep questionnaire, which constitutes a major limitation of our study. However, Fabbrini et al. [48] found no correlation between the items of the Pittsburgh sleep questionnaire and the ESS in patients with PD, suggesting that EDS is not primarily caused by sleep insufficiency but rather by neurodegeneration, e.g. EDS of central origin. Our finding that PD-related fatigue was not associated with frequent arousals during nocturnal sleep nor with a higher PLMS index is also in line with this assumption. A further limitation of this study is the low number of performed polysomnographies that does not allow estimating the influence of sleep apnea on PD-related fatigue and EDS. We found no association between AHI and fatigue or EDS, but the prevalence of moderate to severe sleep apnea was high (54\%). Earlier studies reported prevalences of sleep apnea in PD that ranged between 20-55\% [49-53]. Recently, Monaca et al. [53] could not establish any correlation between AHI and mean sleep latency in the multiple sleep latency test (MSLT).

In conclusion, our findings suggest that fatigue and EDS, although often coexisting, are differently related to severity of motor symptoms, disease duration, depression, and dopaminergic treatment. Fatigue, EDS, and depression all represent frequent non-motor symptoms in PD, which may substantially overlap, in particular fatigue-depression and fatigue-EDS. We assume that - depending on the underlying pathophysiology - fatigue in PD may be influenced by a multitude of factors including vigilance impairment and EDS, depression, and motor symptoms themselves, which probably reflects the coexistent physical and mental dimension of this symptom. Fatigue and EDS should be separately assessed in PD in order to improve 
our understanding of their distinct but overlapping pathophysiology.

\section{References}

1. Friedman JH, Friedman H. Fatigue in Parkinson's disease: a nine-year follow-up. Mov Disord 2001; 16: $1120-1122$.

2. Brañas P, Jordan R, Fry-Smith A, et al. Treatments for fatigue in multiple sclerosis: a rapid and systematic review. Health Technol Assess 2000; 4: 1-61.

3. Zifko UA. Management of fatigue in patients with multiple sclerosis. Drugs 2004; 64: 1295-1304.

4. Choi-Kwon S, Han SW, Kwon SU, et al. Poststroke fatigue: characteristics and related factors. Cerebrovasc Dis 2005; 19: 84-90.

5. Staub F, Bogousslavsky J. Fatigue after stroke: a major but neglected issue. Cerebrovasc Dis 2001; 12: 7581.

6. Baumann CR, Werth E, Stocker R, Ludwig S, Bassetti CL. Sleep-wake disturbances 6 months after traumatic brain injury: a prospective study. Brain 2007; 130: 1873-1883.

7. Frucht S, Rogers JD, Greene PE, Gordon MF, Fahn S. Falling asleep at the wheel: motor vehicle mishaps in persons taking pramipexole and ropinirole. Neurology 1999; 52: 1908-1910.

8. Schapira AH. Sleep attacks (sleep episodes) with pergolide. Lancet 2000; 355: 1332-1333.

9. Hauser RA, Gauger L, Anderson WM, Zesiewicz TA. Pramipexole-induced somnolence and episodes of daytime sleep. Mov Disord 2000; 15: 658-663.

10. Hobson DE, Lang AE, Martin WRW, Razmy A, Rivest J, Fleming J. Excessive daytime sleepiness and suddenonset sleep in Parkinson Disease. JAMA 2002; 287: 455-463.

11. Schenkman M, Wei Zhu C, Cutson TM, Whetten-Goldstein K. Longitudinal evaluation of economic and physical impact of Parkinson's disease. Parkinsonism Relat Disord 2001; 8: 41-50.

12. Shulman LM, Taback RL, Bean J, Weiner WJ. Comorbidity of the nonmotor symptoms of Parkinson's disease. Mov Disord 2001; 16: 507-510.

13. Karlsen K, Larsen JP, Tandberg E, Jørgensen K. Fatigue in patients with Parkinson's disease. Mov Disord 1999; 14: 237-241.

14. Friedman J, Friedman H. Fatigue in Parkinson's disease. Neurology 1993; 43: 2016-2018.

15. Lou JS, Kearns G, Oken B, Sexton G, Nutt J. Exacerbated physical fatigue and mental fatigue in Parkinson's disease. Mov Disord 2001; 16: 190-196.

16. Herlofson K, Larsen JP. Measuring fatigue in patients with Parkinson's disease - the Fatigue Severity Scale. Eur $J$ Neurol 2002; 9: 595-600.

17. Abe K, Takanashi M, Yanagihara T. Fatigue in patients with Parkinson's disease. Behav Neurol 2000; 12: 103-106.

18. Hagell P, Brundin L. Towards an understanding of fatigue in Parkinson disease. $J$ Neurol Neurosurg Psychiatry 2009; 80: 489-493.

19. Alves G, Wentzel-Larsen T, Larsen JP. Is fatigue an independent and persistent symptom in patients with Parkinson disease? Neurology 2004; 63: 1908-1911.
20. Rye DB, Bliwise DL, Dihenia B, Gurecki P. Daytime sleepiness in Parkinson's disease. J Sleep Res 2000; 9: 63-69.

21. Tandberg E, Larsen JP, Karlsen K. Excessive daytime sleepiness and sleep benefit in Parkinson's disease: a community-based study. Mov Disord 1999; 14: 922-927.

22. Adler CH, Thorpy MJ. Sleep issues in Parkinson's disease. Neurology 2005; 64(Suppl.): 12-20.

23. Friedman JH, Chou KL. Sleep and fatigue in Parkinson's disease. Parkinsonism Relat Disord 2004; 10(Suppl.): 2735.

24. Gelb DJ, Oliver E, Gilman S. Diagnostic criteria for Parkinson disease. Arch Neurol 1999; 56: 33-39.

25. Hughes AJ, Ben-Shlomo Y, Daniel SE, Lees AJ. What features improve the accuracy of clinical diagnosis in Parkinson's disease: a clinicopathologic study. Neurology 1992; 42: 1142-1146.

26. Fahn S, Elton RL and the members of the UPDRS Development Committee. Unified Parkinson's Disease Rating Scale. In: Fahn S, Marsden CD, Calne DB, Liebermann A, eds. Recent Development in Parkinson's Disease. Florham Park, NJ: MacMillan Health Care Information, 1987: 153-163.

27. Hoehn MH, Yahr MD. Parkinsonism: onset, progression and mortality. Neurology 1967; 17: 427-442.

28. Valko PO, Bassetti CL, Bloch KE, Held U, Baumann CR. Validation of the Fatigue Severity Scale in a Swiss cohort. Sleep 2008; 31: 1601-1607.

29. Friedman JH, Brown RG, Comella C, et al; as the Working Group on Fatigue in Parkinson's Disease. Fatigue in Parkinson's disease: a review. Mov Disord 2007; 22: 297-308.

30. Bloch KE, Schoch OD, Zhang JN, Russi EW. German version of the Epworth Sleepiness Scale. Respiration 1999; 66: $440-447$.

31. Beck AT, Ward CH, Mendelson M, Mock M, Erbaugh J. An inventory for measuring depression. Arch Gen Psychiatry 1961; 4: 561-571.

32. Razmy A, Lang AE, Shapiro CM. Predictors of impaired daytime sleep and wakefulness in patients with Parkinson Disease treated with older (ergot) vs newer (nonergot) dopamine agonists. Arch Neurol 2004; 61: 97-102.

33. Cronbach LJ. Coefficient alpha and the internal structure of test. Psychometrika 1951; 16: 297-334.

34. Paus S, Brecht HM, Köster J, Seeger G, Klockgether T, Wüllner U. Sleep attacks, dayime sleepiness, and dopamine agonists in Parkinson's disease. Mov Disord 2003; 18: 659-667.

35. Brodsky MA, Godbold J, Roth T, Olanow CW. Sleepiness in Parkinson's disease: a controlled study. Mov Disord 2003; 18: 668-672.

36. Ondo WG, Dat Vuong K, Khan H, Atassi F, Kwak C, Jankovic J. Daytime sleepiness and other sleep disorders in Parkinson's disease. Neurology 2001; 57: 1392-1396.

37. Högl B, Seppi K, Brandauer E, et al. Increased daytime sleepiness in Parkinson's disease: a questionnaire survey. Mov Disord 2003; 18: 319-323.

38. Pittion-Vouyovitch S, Debouverie M, Guillemin F, Vandenberghe N, Anxionnat R, Vespignani H. Fatigue in multiple sclerosis is related to disability, depression and quality of life. J Neurol Sci 2006; 243: 39-45.

39. Bergamaschi R, Romani A, Versino M, Poli R, Cosi V. Clinical aspects of fatigue in multiple sclerosis. Funct Neurol 1997; 12: 247-251. 
40. Flachenecker P, Kümpfel T, Kallmann B, et al. Fatigue in multiple sclerosis: a comparison of different rating scales and correlation to clinical parameters. Mult Scler 2002; 8: 523-526.

41. Gjerstad MD, Aarsland D, Larsen JP. Development of daytime somnolence over time in Parkinson's disease. Neurology 2002; 58: 1544-1546.

42. Del Dotto P, Gambaccini G, Caneparo D, Berti C, Bernardini S, Bonuccelli U. Bedtime cabergoline in Parkinson's disease patients with excessive daytime sleepiness induced by dopamine agonists. Neurol Sci 2003; 24: 170 171.

43. Braak H, Del Tredici K, Rüb U, de Vos RAI, Jansen Steur ENH, Braak E. Staging of brain pathology related to sporadic Parkinson's disease. Neurobiol Aging 2003; 24: 197-211.

44. Fronczek R, Overeem S, Lee SY, et al. Hypocretin (orexin) loss in Parkinson's disease. Brain 2007; 130: 1577 1585.

45. Thannickal TC, Lai YY, Siegel JM. Hypocretin (orexin) cell loss in Parkinson's disease. Brain 2007; 130: 15861595 .

46. Marsh L, McDonald WM, Cummings J, Ravina B. Provisional diagnostic criteria for depression in Parkinson's Disease: a report of an NINDS/NIMH Work Group. Mov Disord 2006; 21: 148-158.
47. Dooneief G, Mirabello E, Bell K, Marder K, Stern Y, Mayeux R. An estimate of the incidence of depression in idiopathic Parkinson's disease. Arch Neurol 1992; 49: 305307.

48. Fabbrini G, Barbanti P, Aurilia C, Vanacore N, Pauletti C, Meco G. Excessive daytime sleepiness in de novo and treated Parkinson's disease. Mov Disord 2002; 17: $1026-1030$.

49. Comella CL. Sleep disorders in Parkinson's disease: an overview. Mov Disord 2007; 17(Suppl.): 367-373.

50. Maria B, Sophia S, Michalis M, et al. Sleep breathing disorders in patients with idiopathic Parkinson's disease. Respir Med 2003; 97: 1151-1157.

51. Arnulf I, Konofal E, Merino-Andreu M, et al. Parkinson's disease and sleepiness: an integral part of PD. Neurology 2002; 58: 1019-1024.

52. Ferini-Strambi L, Franceschi M, Pinto P, Zucconi M, Smirne S. Respiration and heart rate variability during sleep in untreated Parkinson patients. Gerontology 1992; 38: 92-98.

53. Monaca C, Duhamel A, Jacquesson JM, et al. Vigilance trouble in Parkinson's disease: a subjective and objective polysomnographic study. Sleep Med 2006; 7: 448-453. 


\title{
Excessive sleep need following traumatic brain injury: a case-control study of 36 patients
}

\author{
MICHAEL SOMMERAUER, PHILIPP O. VALKO, ESTHER WERTH \\ and CHRISTIAN R. BAUMANN \\ Department of Neurology, University Hospital Zurich, Zurich, Switzerland
}

\author{
Keywords \\ excessive daytime sleepiness, hypersomnia, \\ raumatic brain injury \\ Correspondence \\ Michael Sommerauer, Department of \\ Neurology, University Hospital Zurich, \\ Frauenklinikstrasse 26, 8091 Zurich, \\ Switzerland. \\ Tel.: +41-44-255-55-11; \\ fax: +41-44-255-43-80; \\ e-mail: michael.sommerauer@rwth-aachen.de
}

Accepted in revised form 1 June 2013; received 21 February 2013

DOI: $10.1111 /$ jsr.12068

\begin{abstract}
SUMMARY
Increased sleep need following traumatic brain injury, referred to in this study as post-traumatic pleiosomnia, is common, but so far its clinical impact and therapeutic implications have not been characterized. We present a case-control study of 36 patients with post-traumatic pleiosomnia, defined by an increased sleep need of at least $2 \mathrm{~h}$ per $24 \mathrm{~h}$ after traumatic brain injury, compared to 36 controls. We assessed detailed history, sleep-activity patterns with sleep logs and actigraphy, nocturnal sleep with polysomnography and daytime sleep propensity with multiple sleep latency tests. Actigraphy recordings revealed that traumatic brain injury (TBI) patients had longer estimated sleep durations than controls (10.8 $\mathrm{h}$ per $24 \mathrm{~h}$, compared to $7.3 \mathrm{~h}$ ). When using sleep logs, TBI patients underestimated their sleep need. During nocturnal sleep, patients had higher amounts of slow-wave sleep than controls (20 versus $13.8 \%$ ). Multiple sleep latency tests revealed excessive daytime sleepiness in 15 patients (42\%), and 10 of them had signs of chronic sleep deprivation. We conclude that post-traumatic pleiosomnia may be even more frequent than reported previously, because affected patients often underestimate their actual sleep need. Furthermore, these patients exhibit an increase in slow-wave sleep which may reflect recovery mechanisms, intrinsic consequences of diffuse brain damage or relative sleep deprivation.
\end{abstract}

\section{INTRODUCTION}

Traumatic brain injury (TBI) is a highly prevalent medical condition, particularly in younger people. In the United States, more than 1.5 million people incur TBI every year (RutlandBrown et al., 2006). The available epidemiological data from Europe are in the same range (Bruns and Hauser, 2003; Tagliaferri et al., 2006). Traumatic damage to the brain causes prolonged impairment in many TBI survivors. Sleepwake disturbances are frequent even after mild TBI, and may persist for years (Baumann et al., 2007; Castriotta et al., 2007; Kempf et al., 2010). Six months after trauma, posttraumatic sleep-wake disturbances occur in up to three of four TBI patients (Baumann et al., 2007). Prospective studies in consecutive patients suggest that the most prevalent posttraumatic sleep-wake disturbances are excessive daytime sleepiness (EDS, in 25\%) and excessive sleep need after TBI (in 22\%) (Baumann et al., 2007; Castriotta et al., 2007). The prevalence of insomnia and sleep apnea after TBI needs to be assessed systematically in future studies, but other sleepwake disturbances, including circadian sleep-wake disturbances and parasomnias, are probably less frequent after TBI (Verma et al., 2007). Although recognized as a common complaint, so far the clinical impact and potential therapeutic implications of excessive sleep need in TBI survivors have not been characterized.

In the current literature, the definition of hypersomnia is inconsistent-and is often used interchangeably or synonymous with daytime sleepiness. The word 'hypersomnia' is composed of two different components: the Greek word hyper, i.e. over, exceeding, above, and the Roman somnus, i.e. sleep. Thus, in the narrower sense of the word, hypersomnia stands for an abnormally increased amount of sleep per $24 \mathrm{~h}$ rather than abnormally high sleep pressure. However, the diagnostic criteria of the second edition of the International Classification of Sleep Disorders defines disorders with predominant EDS as hypersomnia disorders (American Academy of Sleep Medicine, 2005). Thus, for 
the sake of clarity, hypersomnia can not be used in patients with excessive sleep need, because the emphasis lies specifically on the quantitative increment of sleep amount irrespective of the presence of absence of daytime sleepiness. However, other terms, such as long sleepers, can also be misleading, because long sleepers are healthy subjects with a sleep need above age-adjusted average values. We therefore suggest introducing a new term for excessive sleep need following TBI: in this study, we will use the term 'post-traumatic pleiosomnia' in patients who need at least $2 \mathrm{~h}$ per $24 \mathrm{~h}$ more sleep compared to their life prior to TBI, irrespective of the presence of EDS (Baumann et al., 2007). The Greek word pleio translates into more, most, full, excessive or multiple.

Our recent studies aimed at assessing the prevalence of various sleep-wake disturbances among TBI patients rather than giving detailed insights into the clinical characteristics of post-traumatic pleiosomnia (Baumann et al., 2007; Kempf et al., 2010). With this study, therefore, we aimed at characterizing post-traumatic pleiosomnia more effectively in a case control study of 36 consecutive and well-defined post-traumatic pleiosomnia patients from our outpatient sleep clinic compared to 36 age- and sex-matched controls.

\section{METHODS}

\section{Subjects}

We included retrospectively consecutive TBI patients who were examined in our tertiary sleep outpatient clinic and who reported chronic post-traumatic pleiosomnia, i.e. an increased sleep need of at least $2 \mathrm{~h}$ per $24 \mathrm{~h}$ compared to pre-TBI conditions, which persisted at least 6 months after trauma. Patients with sleep-wake disorders diagnosed prior to $\mathrm{TBI}$, or with neurological, psychiatric or systemic disorders, were excluded from this study.

As controls, we included subjects without neurological or psychiatric disorders, in whom sleep-wake disorders were excluded by detailed history-taking and sleep laboratory examinations. Subjects with Epworth Sleepiness Scale (ESS) scores above 10, with objective EDS (abnormally decreased mean sleep latencies on multiple sleep latency tests, MSLT) or with apnea-hypopnea indices or periodic limb movement indices above 15 were excluded. Subjects with indices between 5 and 15 were included if history was unremarkable regarding sleep apnea or restless legs syndrome, and if mean sleep latencies on MSLT were normal (above $10 \mathrm{~min}$ ).

All patients gave written informed consent for all study examinations, and the study was approved by the local ethical board.

\section{General assessments}

In our outpatient clinic and sleep laboratory all patients with post-traumatic pleiosomnia underwent a complete diagnostic workup, including the assessment of TBI severity according to the Glasgow Coma Scale (13-15: mild; 9-12: moderate; 3-8: severe), and computed tomography images after trauma were screened for intracranial bleedings. Every patient was given a detailed neurological evaluation, and outcome after TBI was assessed along the Glasgow Outcome Scale which we used in our previous study (Baumann et al., 2007). The scale classifies outcome as follows: (1) dead, (2) vegetative state, (3) severe disability, i.e. able to follow commands, but unable to live independently, (4) moderate disability, i.e. able to live independently, but unable to return to work or school, and (5) good recovery, i.e. able to return to work or school. Medical treatment need was ascertained.

In 15 TBI patients, we measured cerebrospinal fluid hypocretin-1 levels by radioimmunoassay. Cerebrospinal fluid was obtained by lumbar puncture as described earlier, and according to our local reference samples, hypocretin-1 levels below $320 \mathrm{pg} \mathrm{mL}^{-1}$ were considered as abnormally low (Baumann et al., 2005).

\section{Assessments of sleep and wakefulness}

We applied subjective measurements of sleepiness by the ESS (Bloch et al., 1999; Johns, 1991), and of fatigue by the Fatigue Severity Scale (FSS) (Valko et al., 2008). Subjective EDS was diagnosed when ESS scores were 10 or above, and fatigue in patients with scores above 4.0. In addition, we assessed recordings of sleep and wakefulness including actigraphy, nocturnal polysomnography (PSG) and MSLT.

All sleep laboratory recordings were performed without sleep-wake modulating drugs; i.e. after washout of drugs if necessary. Prior to sleep laboratory tests, we recorded sleep and physical activity levels over 14 days with sleep logs and wrist actigraphy (on the non-dominant wrist; light sensor data included; Actiwatch, Neurotechnology, Cambridge, UK) (Morgenthaler et al., 2007). Actigraphy was used to determine periods of inactivity per $24 \mathrm{~h}$ to gain an estimate of sleep duration, and to screen for circadian sleep-wake disorders. In addition, we checked whether daytime naps showed a circadian distribution; i.e. whether they occurred more often in the morning, after lunch or in the afternoon.

Conventional nocturnal video-PSG (using a 16-channel recording system: Embla A10; Embla, Colorado, CO, USA) was performed overnight. Two patients underwent an additional PSG ad libitum; i.e. patients were not woken up in the morning and thus given the opportunity to sleep as long as they could. These additional PSGs were not followed by another MSLT. Sleep stage scoring was performed along standard criteria (lber et al., 2007). Polysomnography was performed as reported before, and scoring as well as clinical classification was performed along actual guidelines (American Academy of Sleep Medicine, 2005; Baumann et al., 2007).

The MSLT consisted of four sleep opportunities every $2 \mathrm{~h}$, measuring EDS objectively under standard conditions and in the absence of external alerting factors (Littner et al., 2005). Sleep latencies on MSLT were defined as the time from lights-off to the first epoch of any sleep stage. Objective 
excessive daytime sleepiness was diagnosed when mean sleep latency was $<8 \mathrm{~min}$.

\section{Statistical analyses}

Statistical analyses were performed using spss version 19.0 (IBM, Zurich, Switzerland). Group data are described by means and standard deviations (SD). For univariate analysis, we used one-way analysis of variance (ANOVA) for numerical scale variables and $\chi^{2}$-test for nominal scale variables. For correlation analysis we used a two-way Pearson's product-moment correlation coefficient. Multiple linear regression analyses were performed to assess the relationship between sleep/inactivity times per $24 \mathrm{~h}$ (dependent variable) and age, sex, severity of TBI and clinical outcome (Glasgow Coma Scale).

\section{RESULTS}

\section{Demographic and clinical characterization}

We identified $36 \mathrm{TBI}$ patients who met the inclusion criteria for post-traumatic pleiosomnia and 36 age- and sex-matched controls (Tables 1 and 2). Fourteen TBI patients have been reported before (Baumann et al., 2007). Twenty-four patients returned to work after their trauma. An overview on TBI characteristics and clinical outcomes is given in Table 1. Mean levels of excessive daytime sleepiness and fatigue, as assessed subjectively by questionnaires, were similar in both groups. Thirteen TBI patients and no controls scored ESS above 10, indicating subjective daytime sleepiness. Symptoms of restless legs syndrome were not reported by patients or by controls. Epilepsy was not present in any TBI patient, and no patient was treated with stimulants before or during sleep recordings.

\section{Actigraphy findings}

All patients complained about increased sleep need of more than $2 \mathrm{~h}$ after TBI (mean difference: $2.4 \mathrm{~h}$, range 2.0-6.5 h).

\begin{tabular}{|c|c|}
\hline \multicolumn{2}{|l|}{ Characteristics of traumatic brain injury } \\
\hline Severity of TBI (mild-moderate-severe) & $13-7-16$ \\
\hline Intracranial bleedings & $18(50 \%)$ \\
\hline Interval since TBI (months) & $32 \pm 18$ \\
\hline \multicolumn{2}{|l|}{ Outcome after traumatic brain injury } \\
\hline Glasgow Outcome Scale (1-2-3-4-5) & $0-0-0-13-23$ \\
\hline Headache since trauma & $20(56 \%)$ \\
\hline Worse when not having slept enough & $13(65 \%$ of 20$)$ \\
\hline Report of cognitive problems since trauma & $14(39 \%)$ \\
\hline Worse when not having slept enough & $12(86 \%$ of 14$)$ \\
\hline Motor deficits since trauma & $4(11 \%)$ \\
\hline Sensory deficits since trauma & $9(25 \%)$ \\
\hline Cranial nerve deficits since trauma & $5(14 \%)$ \\
\hline Pain other than headache since trauma & $3(8 \%)$ \\
\hline
\end{tabular}

According to sleep logs, mean time asleep after TBI was $9.4 \mathrm{~h}$ compared to $7.5 \mathrm{~h}$ in controls $(P=0.008$, Table 2$)$. Interestingly, objective measures obtained by actigraphy revealed a more pronounced increase of estimated sleep need, as indicated by significantly prolonged periods of inactivity (mean $10.8 \mathrm{~h}$ ) compared to controls (mean $7.3 \mathrm{~h}$; $P<0.001)$. The difference of mean time asleep/inactive between sleep logs and actigraphy was significant $(P=0.02)$ in TBI patients, but not in controls. Estimated sleep duration $\geq 10 \mathrm{~h}$ per $24 \mathrm{~h}$ was observed in 21 patients (58\%), but in no controls $(P<0.001)$. Sleep logs and actigraphy recordings revealed regular (at least five per week) daytime naps in 17 patients $(47 \%)$ and in none of the controls $(P<0.001)$. These daytime naps did not reveal a clear circadian distribution.

\section{Polysomnography and MLST}

Sleep laboratory findings are summarized in Table 2. Sleep duration and sleep efficiency were similar in both groups. Slow-wave sleep [non-rapid eye movement (NREM) stage 3] accounted for $20.4 \%$ of recorded sleep in patients and for $13.8 \%$ in controls $(P=0.009)$. The amount of other sleep stages did not differ between patients and controls. Also, wake after sleep onset in the percentage of sleep period time, periodic limb movement indices and apnea-hypopnea indices were similar in TBI patients and in controls, and the latter were not linked to arousals. Parasomnia disorders were not observed.

During ad libitum PSG, two patients had sleep durations of 798 and $831 \mathrm{~min}$; i.e. 13.3 and 13.9 h. Sleep efficiencies were 90 and $84 \%$, and slow-wave sleep accounted for 21 and $13 \%$. Actigraphy recordings suggested that both patients were sleep-satiated before PSG.

Multiple sleep latency tests revealed objective EDS in 15 patients $(42 \%)$ and in no controls $(0 \%, P<0.001)$. In 10 patients with objective EDS, as defined by a mean sleep latency of $\leq 8 \mathrm{~min}$, actigraphy revealed that nocturnal sleep periods were at least $2 \mathrm{~h}$ shorter during weekdays than during weekends. All 10 patients returned to work. This actigraphy pattern was also found in two TBI patients without objective EDS. Mean sleep latencies were $8.7 \mathrm{~min}$ in patients and $12.4 \mathrm{~min}$ in controls $(P<0.001)$. Multiple sleep onset REM periods were observed in three patients and in no controls [not significant (NS)]. In both groups, mean sleep latencies on MSLT were not correlated with PSG findings, such as sleep efficiency or total sleep time.

\section{Clinical correlates of sleep findings}

Longer intervals between TBI and sleep recordings were associated with longer REM sleep latencies $(r=0.52$, $P=0.01)$. Other polysomnography parameters such as deep sleep were not associated with time interval since injury. Furthermore, severity of $\mathrm{TBI}$ or the presence of intracranial bleedings were not associated with any sleep-wake finding, 
Table 2 Demographic data, sleep-wake measures and cerebrospinal fluid hypocretin levels in 36 patients with traumatic brain injury, compared to 36 controls

\begin{tabular}{|c|c|c|c|}
\hline & Patients $(\mathrm{n}=36)$ & Controls $(\mathrm{n}=36)$ & $\mathrm{P}$ \\
\hline \multicolumn{4}{|l|}{ Demographic data } \\
\hline Sex (male) & $26(72 \%)$ & $26(72 \%)$ & NS \\
\hline Age (years) & $36 \pm 12$ & $36 \pm 10$ & NS \\
\hline \multicolumn{4}{|l|}{ Hypocretin (orexin) } \\
\hline Cerebrospinal levels $\left(\mathrm{pg} \mathrm{mL}^{-1}\right)$ & $456 \pm 181(n=15)$ & $497 \pm 63(n=20)^{\dagger}$ & NS \\
\hline Cerebrospinal levels low $\left(<320 \mathrm{pg} \mathrm{mL}^{-1}\right)$ & $3(20 \%)$ & $0(0 \%)$ & NS \\
\hline \multicolumn{4}{|l|}{ Sleepiness and fatigue } \\
\hline Epworth sleepiness scale (ESS) & $8.7 \pm 4.9$ & $7.1 \pm 3.8$ & NS \\
\hline ESS score $\geq 10$ & $13(36 \%)$ & $0(0 \%)$ & $<0.001$ \\
\hline Fatigue severity scale & $3.7 \pm 1.0$ & $3.2 \pm 1.2$ & NS \\
\hline \multicolumn{4}{|l|}{ Estimated sleep need per $24 \mathrm{~h}$} \\
\hline Actigraphy: estimated sleep per $24 \mathrm{~h}(\mathrm{~h})$ & $10.8(8.0-15.6)$ & $7.3(5.7-9.2)$ & $<0.001$ \\
\hline$\geq 10 \mathrm{~h}$ estimated sleep per $24 \mathrm{~h}(\mathrm{~h})$ & $21(58 \%)$ & $0(0 \%)$ & $<0.001$ \\
\hline Sleep logs: sleep per $24 h(h)$ & $9.4(6.8-15.0)$ & $7.5(6.1-9.3)$ & 0.008 \\
\hline Actigraphy/sleep log: regular daytime naps & $17(47 \%)$ & $0(0 \%)$ & $<0.001$ \\
\hline \multicolumn{4}{|l|}{ Nocturnal video-polysomnography } \\
\hline Mean sleep duration (min) & $379(316-476)$ & $375(250-481)$ & NS \\
\hline Sleep efficiency $(\%)$ & $90.2(66.1-99.6)$ & $86.9(66.6-98.5)$ & NS \\
\hline Wake after sleep onset ( $\%$ of SPT) & $5.3(0.3-29.5)$ & $6.5(1.1-30.3)$ & NS \\
\hline NREM1 (\%) & $5.0(2.4-8.2)$ & $5.9(2.8-10.0)$ & NS \\
\hline NREM2 (\%) & $44.8(31.5-64.2)$ & $50.1(38.6-65.8)$ & NS \\
\hline Slow-wave sleep (NREM3) (\%) & $20.4(11.9-38.0)$ & $13.8(0-26.9)$ & 0.009 \\
\hline REM sleep (\%) & $20.1(4.5-34.8)$ & $18.1(7.1-26.1)$ & NS \\
\hline Sleep latency $(\mathrm{min})$ & $22.9(3.6-68)$ & $19.9(3.5-87)$ & NS \\
\hline REM sleep latency (min) & $88.9(4.9-302.5)$ & $112.6(46.5-288.5)$ & NS \\
\hline Apnea-hypopnea index $\left(\mathrm{h}^{-1}\right)$ & $4.5(0-14.5)$ & $3.2(0-14.9)$ & NS \\
\hline PLMS index $\left(\mathrm{h}^{-1}\right)$ & $3.2(0-13.4)$ & $1.9(0-8.9)$ & NS \\
\hline \multicolumn{4}{|l|}{ Multiple sleep latency test } \\
\hline Mean sleep latency (min) & $8.7(2.9-17.2)$ & $12.4(8.1-18.1)$ & $<0.001$ \\
\hline Mean sleep latency $<8 \mathrm{~min}$ & $15(42 \%)$ & $0(0 \%)$ & $<0.001$ \\
\hline$\geq 2$ sleep onset REM periods & $3(8 \%)$ & $0(0 \%)$ & NS \\
\hline
\end{tabular}

NS, not significant; PLMS, periodic limb movements during sleep; REM sleep, rapid eye movement sleep; SPT, sleep period time.

${ }^{\dagger}$ Normative cerebrospinal fluid levels from 20 previously published controls as measured in our laboratory (Baumann et al., 2005).

including sleep duration. Cerebrospinal fluid hypocretin-1 determination was performed in 15 patients, but levels were not associated with any actigraphy, PSG or MSLT findings. However, three patients had a pathologically low hypocretin1 level $\left(118 / 278 / 301 \mathrm{pg} \mathrm{mL}^{-1}\right)$. All three patients suffered from severe TBI with intracranial haemorrhage. ESS scores were normal in all of them, but MSLT revealed EDS in two patients (hypocretin: 278 and $301 \mathrm{pg} \mathrm{mL}^{-1}$, respectively). Multiple sleep onset REM periods were found in two of these patients (118 and $301 \mathrm{pg} \mathrm{mL}^{-1}$ ). Actigraphic measures of sleep per $24 \mathrm{~h}$ were not different from TBI patients with normal hypocretin levels.

Regression analyses showed that age, sex, severity of TBI and clinical outcome were not related to sleep times per $24 \mathrm{~h}$. Furthermore, we compared post-traumatic pleiosomnia patients with sleep times over $10 \mathrm{~h}(n=21)$ to those with less than $10 \mathrm{~h}$ sleep per $24 \mathrm{~h}(n=15)$. The two groups did not differ in terms of age, sex, severity of TBI, clinical outcome (Glasgow Outcome Scale), Epworth Sleepiness Scale or fatigue severity scale scores, PSG measures such as sleep efficiency, sleep latency, percentages of any sleep stage or mean sleep latencies on MSLT.

\section{DISCUSSION}

Post-traumatic pleiosomnia is a common condition following $\mathrm{TBI}$, and persists for many years (Baumann et al., 2007; Kempf et al., 2010). We define this condition as an increased sleep need per $24 \mathrm{~h}$ of at least $2 \mathrm{~h}$ compared to the time before trauma. Until now, however, there exists neither a characterization of this neglected condition, nor do we know how to treat it. Thus, this case-control study was designed to shed some light into the clinical characteristics of posttraumatic pleiosomnia.

Patients who suffered from TBI did not estimate their actual sleep need correctly. In sleep logs, mean reported total sleep time per day was more than $1 \mathrm{~h}$ shorter than the estimated sleep duration calculated by duration of inactivity measured in the actigraphy studies (10.8 h). Furthermore, 6 months after trauma, we observed in our previous study on post-traumatic sleep-wake disturbances a tendency to underestimate sleep propensity during daytime (Baumann et al., 2007). In the present study, with an interval of $32 \pm 18$ months after trauma, we found subjective EDS-as assessed with ESS-in 13 patients and objective EDS-as assessed with MSLT-in 
15 patients. Altogether, these findings re-emphasize an apparently common yet widely neglected phenomenon, namely that underestimation or anosognosia of post-traumatic sleep-wake disturbances seems to be frequent among TBI survivors. The present study confirms our previous observation that TBI patients may not be aware of their increased sleep pressure, which is diagnosed only when performing a multiple sleep latency test (Baumann et al., 2007). In addition, a similar underestimation occurs also with respect to sleep need per $24 \mathrm{~h}$, as indicated by the discrepancy between sleep logs and estimated sleep duration by actigraphy. Based on this novel observation, it appears likely that our history-based screening method for patient inclusion was not sensitive enough. Consequently, post-traumatic pleiosomnia might be even more prevalent than in $17 \%$ of TBI patients, as found in our previous study on the prevalence of post-traumatic sleep-wake disturbances (Baumann et al., 2007). This possibility must be considered when counselling and treating $\mathrm{TBI}$ patients.

Objective EDS was present in only $42 \%(n=15)$ of TBI patients with post-traumatic pleiosomnia. Thus, EDS is not a mandatory criterion for the diagnosis of post-traumatic pleiosomnia. Conversely, in 10 of 15 post-traumatic pleiosomnia patients with EDS, actigraphy studies displayed signs of chronic sleep deprivation; i.e. the need to catch up sleep during weekends. Such a pattern was found particularly in young and middle-aged patients who are integrated in tight work schedules or have families with young children. This suggests that patients whose psychosocial environment allows sleeping longer per $24 \mathrm{~h}$ could avoid increased sleep propensity at daytime, whereas patients who cannot compensate for the increased sleep need suffer consecutively from EDS. Therefore, we might speculate that post-traumatic pleiosomnia is a primary sleep-wake disturbance following TBI, whereas EDS might be secondary to relative sleep deprivation.

During polysomnography recordings we observed increased slow-wave sleep in patients with post-traumatic pleiosomnia, even many months after trauma. The reason for increased deep sleep remains speculative: on one hand, it might be caused by brain damage itself, or it might play a role in regeneration and neuroplasticity. Early studies revealed that damage to both the brainstem and the hypothalamus-both important structures for arousal-are common after TBI (Crompton, 1971a,b). Accordingly, we found partial loss of hypocretin neurones in the hypothalamus of deceased TBI patients (Baumann et al., 2009). On the other hand, the finding of low hypocretin-1 levels in the cerebrospinal fluid is rare in sleepy TBI patients. We know that normal cerebrospinal fluid hypocretin-1 levels might not reflect partial hypocretin neuronal loss, but deficient hypocretin signalling is probably not the explanation for posttraumatic pleiosomnia and increased slow-wave sleep (Fronczek et al., 2007; Gerashchenko et al., 2003). It appears more likely that increased sleep need is guided by damage to several sleep-wake modulating neuronal systems. TBI, with its focal impact and diffuse parenchymatous damage, is disposed to affect widespread neuronal systems in the brain.

In contrast, it is conceivable that increased sleep need and particularly increased slow-wave sleep following TBI might reflect recovery processes, particularly axonal sprouting, synaptic remodelling and short-term plasticity processes (Carmichael and Chesselet, 2002; Dijk, 2009; Stickgold et al., 2001). The beneficial effect of sleep and slow-wave sleep on recovery has been shown in several studies on rodents. Rats, which underwent sleep deprivation after stroke, showed an increase in stroke size as well as delayed motor recovery (Gao et al., 2010; Zunzunegui et al., 2011). Conversely, the drug gamma-hydroxybutyrate, which is known to enhance slow-wave sleep, accelerates functional recovery after stroke (Gao et al., 2008). In this regard, however, it would be somewhat surprising if these recovery adaptation mechanisms lasted so long; i.e. for many years after trauma. Finally, it is conceivable that increased slowwave sleep reflects, at least in some patients, a relative sleep insufficiency.

Post-traumatic pleiosomnia can persist for many years. In a recent study, we found post-traumatic pleiosomnia in $27 \%$ of 51 consecutive TBI patients 3 years after trauma (Kempf et al., 2010). In the present series, the longest follow-up time was 93 months. Our society, however, demands full activity at work-patients with an increased sleep need are disadvantaged and perhaps compensate for their increased sleep time only during their leisure time. Thus, due to the chronic course of post-traumatic pleiosomnia, medical treatment would be necessary to allow patients returning to their normal lives. Until now, however, medical treatment of posttraumatic pleiosomnia remains an unsolved issue (Kaiser et al., 2010).

This study has limitations. First, due to the retrospective character of this study in consecutive patients, the age range of TBI patients is high, and additional data such as psychometric evaluation and neuroimaging results are not available. Furthermore, the number of included patients is low, because we aimed at including only well-described patients. Therefore, we cannot exclude a selection bias in our study. Narrowing the age range would have reduced the number of patients too much, and regression analyses showed that age was not associated with pleiosomnia. Secondly, we did not control for concomitant depression which might impact fatigue and even sleep need and inactivity, which is measured by actigraphy. This should be conducted in future studies. As actigraphy can only give an estimation of sleep duration, future studies could also investigate electroencephalographic sleep-wake measures and parameters of sleep homeostasis across $24 \mathrm{~h}$ and over several days. Thus, it is conceivable that novel diagnostic tools such as continuous polysomnography during $24 \mathrm{~h}$ might provide additional insights and lead ultimately to the better diagnosis of hypersomnia disorders, including post-traumatic pleiosomnia (Pizza et al., 2013). 
In conclusion, post-traumatic pleiosomnia is probably even more frequent than reported previously, and can be associated with EDS, particularly in patients who cannot allow themselves to sleep enough. Furthermore, post-traumatic pleiosomnia patients exhibit an increase in slow-wave sleep, which might reflect recovery mechanisms or intrinsic consequences of diffuse brain damage. Whether or not it makes sense to define post-traumatic pleiosomnia with and without long sleep time (such as in idiopathic hypersomnia) will be examined based on the study of larger numbers of patients. The preliminary results of this study revealed no differences between these two groups.

\section{ACKNOWLEDGEMENTS}

This work was supported by the Swiss National Science Foundation (SNF; 32003B-125504 to C.B.; www.snf.ch) and an unrestricted grant of the Schweizerischer Versicherungsverband (SVV). The SNF is an independent national nonprofit funding organization. The funders had no role in study design, data collection and analysis, decision to publish, or preparation of the manuscript.

\section{AUTHOR CONTRIBUTIONS}

MS and CB contributed to the design, initiation and management of the project and writing of the manuscript. EW performed in-depth analyses of electrophysiological recordings (actigraphy, polysomnography, multiple sleep latency tests). PV contributed to writing of the manuscript.

\section{CONFLICT OF INTEREST}

No conflicts of interest declared.

\section{REFERENCES}

American Academy of Sleep Medicine. ICSD-2-International Classification of Sleep Disorders, 2nd edn. Diagnostic and Coding Manual. American Academy of Sleep Medicine, Westchester, IL, 2005.

Baumann, C. R., Stocker, R., Imhof, H. G., et al. Hypocretin-1 (orexin A) deficiency in acute traumatic brain injury. Neurology, 2005, 65: 147-149.

Baumann, C. R., Werth, E., Stocker, R., Ludwig, S. and Bassetti, C. L. Sleep-wake disturbances 6 months after traumatic brain injury: a prospective study. Brain, 2007, 130: 1873-1883.

Baumann, C. R., Bassetti, C. L., Valko, P. O., et al. Loss of hypocretin (orexin) neurons with traumatic brain injury. Ann. Neurol., 2009, 66: 555-559.

Bloch, K. E., Schoch, O. D., Zhang, J. N. and Russi, E. W. German version of the Epworth Sleepiness Scale. Respiration, 1999, 66: 440-447.

Bruns, J. Jr and Hauser, W. A. The epidemiology of traumatic brain injury: a review. Epilepsia, 2003, 44(Suppl. 10): 2-10.
Carmichael, S. T. and Chesselet, M. F. Synchronous neuronal activity is a signal for axonal sprouting after cortical lesions in the adult. J. Neurosci., 2002, 22: 6062-6070.

Castriotta, R. J., Wilde, M. C., Lai, J. M., Atanasov, S., Masel, B. E. and Kuna, S. T. Prevalence and consequences of sleep disorders in traumatic brain injury. J. Clin. Sleep Med., 2007, 3: 349-356.

Crompton, M. R. Brainstem lesions due to closed head injury. Lancet, 1971a, 1: 669-673.

Crompton, M. R. Hypothalamic lesions following closed head injury. Brain, 1971b, 94: 165-172.

Dijk, D. J. Regulation and functional correlates of slow wave sleep. J. Clin. Sleep Med., 2009, 5: S6-S15.

Fronczek, R., Overeem, S., Lee, S. Y., et al. Hypocretin (orexin) loss in Parkinson's disease. Brain, 2007, 130: 1577-1585.

Gao, B., Kilic, E., Baumann, C. R., Hermann, D. M. and Bassetti, C. L. Gamma-hydroxybutyrate accelerates functional recovery after focal cerebral ischemia. Cerebrovasc. Dis., 2008, 26: 413-419.

Gao, B., Cam, E., Jaeger, H., Zunzunegui, C., Sarnthein, J. and Bassetti, C. L. Sleep disruption aggravates focal cerebral ischemia in the rat. Sleep, 2010, 33: 879-887.

Gerashchenko, D., Murillo-Rodriguez, E., Lin, L., et al. Relationship between CSF hypocretin levels and hypocretin neuronal loss. Exp. Neurol., 2003, 184: 1010-1016.

Iber, C., Ancoli-Israel, S., Chesson, A. and Quan, S. F. The AASM Manual for the Scoring of Sleep and Associated Events: Rules, Terminology, and Technical Specification, 1st edn. American Academy of Sleep Medicine, Westchester, IL, 2007.

Johns, M. W. A new method for measuring daytime sleepiness: the Epworth Sleepiness Scale. Sleep, 1991, 14: 540-545.

Kaiser, P. R., Valko, P. O., Werth, E., et al. Modafinil ameliorates excessive daytime sleepiness after traumatic brain injury. Neurology, 2010, 75: 1780-1785.

Kempf, J., Werth, E., Kaiser, P. R., Bassetti, C. L. and Baumann, C. R. Sleep-wake disturbances 3 years after traumatic brain injury. J. Neurol. Neurosurg. Psychiatry, 2010, 81: 1402-1405.

Littner, M. R., Kushida, C., Wise, M., et al. Practice parameters for clinical use of the multiple sleep latency test and the maintenance of wakefulness test. Sleep, 2005, 28: 113-121.

Morgenthaler, T., Alessi, C., Friedman, L., et al. Practice parameters for the use of actigraphy in the assessment of sleep and sleep disorders: an update for 2007. Sleep, 2007, 30: 519-529.

Pizza, F., Moghadam, K. K., Vandi, S., et al. Daytime continuous polysomnography predicts MSLT results in hypersomnias of central origin. J. Sleep Res., 2013, 22: 32-40.

Rutland-Brown, W., Langlois, J. A., Thomas, K. E. and Xi, Y. L. Incidence of traumatic brain injury in the United States, 2003. J. Head Trauma Rehabil., 2006, 21: 544-548.

Stickgold, R., Hobson, J. A., Fosse, R. and Fosse, M. Sleep, learning, and dreams: off-line memory reprocessing. Science, 2001, 294: 1052-1057.

Tagliaferri, F., Compagnone, C., Korsic, M., Servadei, F. and Kraus, J. A systematic review of brain injury epidemiology in Europe. Acta Neurochir. (Wien), 2006, 148: 255-268. discussion 68.

Valko, P. O., Bassetti, C. L., Bloch, K. E., Held, U. and Baumann, C. R. Validation of the fatigue severity scale in a Swiss cohort. Sleep, 2008, 31: 1601-1607.

Verma, A., Anand, V. and Verma, N. P. Sleep disorders in chronic traumatic brain injury. J. Clin. Sleep Med., 2007, 3: 357-362.

Zunzunegui, C., Gao, B., Cam, E., Hodor, A. and Bassetti, C. L. Sleep disturbance impairs stroke recovery in the rat. Sleep, 2011, 34: 1261-1269. 
Original Article

\title{
Multiple sleep latency measures in narcolepsy and behaviourally induced insufficient sleep syndrome
}

\author{
Isabelle Marti ${ }^{1}$, Philipp O. Valko ${ }^{*, 1}$, Ramin Khatami, Claudio L. Bassetti, Christian R. Baumann \\ Department of Neurology, University Hospital of Zurich, 8091 Zurich, Switzerland
}

\section{A R T I C L E I N F O}

\section{Article history:}

Received 1 September 2008

Received in revised form 5 February 2009

Accepted 5 March 2009

Available online 21 May 2009

\section{Keywords}

Narcolepsy-cataplexy

Behaviourally induced insufficient sleep

syndrome

Multiple sleep latency test

Sleep stage sequencing

Sleep onset REM period

Excessive daytime sleepiness

\begin{abstract}
A B S T R A C T
Background: Short mean latencies to the first epoch of non-rapid eye movement sleep stage 1 (NREM1) and the presence of $\geqslant 2$ sleep onset REM (SOREM) periods on multiple sleep latency test (MSLT) occur in both narcolepsy-cataplexy (NC) and behaviourally induced insufficient sleep syndrome (BIISS). It is not known whether specific MSLT findings help differentiate the two disorders.

Methods: We analyzed MSLT data including sleep latencies to and between different sleep stages of 60 age-, gender- and body mass index (BMI)-matched subjects (hypocretin-deficient NC, actigraphyconfirmed BIISS, healthy controls: each 20).

Results: Mean latency (in minutes) to NREM1 sleep was significantly shorter in NC $(1.8 \pm 1.5)$ than in BIISS $(4.7 \pm 2.1, p<0.001)$ and controls $(11.4 \pm 3.3, p<0.001)$. Mean latency to NREM2 sleep was similar in NC $(8.6 \pm 4.7)$ and BIISS $(8.1 \pm 2.7, p=0.64)$; latency to either NREM2 or rapid eye movement (REM) sleep (i.e., the sum of the sleep latency to NREM1 and the duration of the first NREM1 sleep sequence), however, was shorter in NC $(4.4 \pm 2.9)$ than in BIISS $(7.9 \pm 3.5, p<0.001)$. Referring to all naps with SOREM periods, the sequence NREM1-REM-NREM2 was more common (71\%) in NC than in BIISS $(15 \%, p<0.001)$, reflecting the shorter latency from NREM1 to NREM2 in BIISS $(3.7 \pm 2.5)$ than in NC $(6.1 \pm 5.9, p<0.001)$.

Conclusions: Our findings show that both sleepiness (as measured by NREM1 sleep latency) and REM sleep propensity are higher in NC than in BIISS. Furthermore, our finding of frequent REM sleep prior to NREM2 sleep in NC is in line with the recent assumption of an insufficient NREM sleep intensity in NC. Together with detailed clinical interviews, sleep logs, actigraphy, and nocturnal polysomnography, mean sleep latencies to NREM1 $\leqslant 2.5 \mathrm{~min}$, the presence of multiple SOREM periods, and the sequence NREM1-REM-NREM2 may be the best MSLT measures to discriminate NC from BIISS.
\end{abstract}

(c) 2009 Elsevier B.V. All rights reserved.

\section{Introduction}

Excessive daytime sleepiness (EDS) represents the leading symptom of narcolepsy, along with cataplexy, sleep paralysis, and hypnagogic hallucinations. The pathophysiological hallmark of narcolepsy with cataplexy (NC) is the loss of hypothalamic neurons that produce wake-promoting neuropeptides, the hypocretins (orexins). Besides the assessment of a detailed history, the multiple sleep latency test (MSLT) is the most important ancillary test for the diagnosis of NC, together with the measurement of cerebrospinal fluid hypocretin levels. The typical MSLT findings for narcolepsy include (1) a mean sleep latency of $\leqslant 8 \mathrm{~min}$ and (2) the presence of $\geqslant 2$ sleep onset rapid eye movement (SOREM) periods as an indicator of high REM sleep propensity [1-3]. However, these findings are not specific for narcolepsy since other sleep-wake

\footnotetext{
* Corresponding author. Tel.: +41 4425555 11; fax: +41 442554380 . E-mail address: philipp.valko@usz.ch (P.O. Valko).

1 Both authors have equally contributed to this work.
}

disorders with EDS such as sleep apnea syndrome or behaviourally induced insufficient sleep syndrome (BIISS; also referred to as chronic sleep deprivation) may present with similar MSLT results $[2,4]$.

There is considerable evidence of a continuous reduction in average sleep time by $20 \%$ over the past century [5]. Due to professional or social obligations, people often are not able or do not allow themselves to sleep enough. Besides EDS, BIISS may be associated with decreased neurocognitive functioning, fatigue, increased risk of motor vehicle crashes, negative mood states, and decreased motivation [6]. Accordingly, BIISS is a frequent and important differential diagnosis of NC in clinical practice: general practitioners and neurologists often refer BIISS patients to sleep laboratories with the suspicion of narcolepsy. For sleep specialists who are familiar with NC patients, differentiating between NC and BIISS is often possible on clinical grounds alone. First, clear-cut cataplexy is pathognomonic for NC and does not occur in BIISS patients. However, cataplexy-like symptoms, sleep paralysis, and hallucinations are occasionally found in the setting of non-narcoleptic EDS 
patients [7]. Second, BIISS-related EDS can be as irresistible as in NC (e.g., microsleep during car driving), and short episodes of sleep are also usually refreshing (e.g., power naps for sleep-deprived hospital doctors). In terms of objective measures of EDS, very low mean sleep latencies on MSLT and polysomnography recordings are found in both BIISS and NC patients [8]. Last but not least, NREM sleep is better preserved than REM sleep during sleep restriction, possibly leading to high REM sleep propensity $[9,10]$. Indeed, SOREM periods are also observed in BIISS patients [2,4].

To better distinguish MSLT findings in NC and in BIISS, we aimed at characterizing the sleep latencies to and between different sleep stages in age-, gender- and BMI-matched patients with definite NC, BIISS, and normal controls.

\section{Patients and methods}

\subsection{Subjects}

Among patients referred to the Sleep Disorder Centre of the Neurology Department of the University Hospital of Zurich, we retrospectively selected 3 groups (NC, BIISS, and controls), each consisting of 20 age-, gender- and BMI-matched patients. EDS was present in all patients and was estimated by the Epworth sleepiness scale (ESS). In all NC patients, the diagnosis was confirmed by deficient cerebrospinal fluid hypocretin levels. In order to minimize concomitant BIISS, NC patients were instructed to pay regard to an optimal sleep hygiene including sufficient sleep amount, regular sleep-wake habits and repeated short daytime naps. Actigraphic recordings in NC patients confirmed the absence of chronic sleep restriction (i.e., reduced sleep time per $24 \mathrm{~h}$ ). The diagnosis of BIISS was made according to the international classification of sleep disorders, including improvement of subjective EDS after sleep prolongation as documented by sleep logs and actigraphy [3]. Patients with additional sleep-wake disorders (e.g., sleep apnea syndrome) were excluded. Control subjects did not report EDS (normal ESS scores), nor did they suffer from any kind of sleep-wake disorder. Patients and controls were free from drugs affecting the central nervous system, except for two narcoleptic patients (modafinil and sodium valproate, respectively), one BIISS patient (citalopram), and two controls (phenytoin/gabapentin and citalopram, respectively).

\subsection{Sleep studies}

All patients underwent videopolysomnographic recording in the night prior to the MSLT. MSLT was done according to standard guidelines [11]. Briefly, patients underwent four or five naps, each lasting $20 \mathrm{~min}$, at $2 \mathrm{~h}$ intervals over the day. If sleep occurred, tests were run for another $15 \mathrm{~min}$ to assess SOREM periods. The MSLT montage included central (C3 and C4) and occipital (01 and O2) electrodes with auricular reference electrodes, two electrooculographic channels, two submental electromyographic channels, and electrocardiography (ECG). Sleep stages were scored using 30-second epochs according to standard criteria by Rechtschaffen and Kales [12].

We measured sleep latencies from lights-off to the first epoch of non-rapid eye movement stage 1 (NREM1), NREM2, and REM sleep. Furthermore, we assessed the duration of specific sleep stages. In naps without sleep, mean sleep latency was defined as $20 \mathrm{~min}$.

\subsection{Statistics}

Statistical analyzes were performed using SPSS statistical analysis program (SPSS 12.0). Data are reported as means \pm standard deviation if not otherwise indicated. Comparisons between NC,
BIISS and controls were made using one-way ANOVA. For direct comparisons between NC and BIISS, we used independent $t$-test and Chi-square statistics. Significance level was assumed for $p$ values $<0.05$. Significant result on Chi-square and $t$-tests are only presented when one-way ANOVA revealed differences between the three groups ( $F$-values and $p$ levels of ANOVA are not reported).

\section{Results}

\subsection{Subjects}

The demographic and clinical characteristics of the three groups are summarized in Table 1. Subjective daytime sleepiness (estimated by the ESS) was similar between NC and BIISS patients ( $16 \pm 4$ and $15 \pm 3$, respectively, $p=0.2$ ) and significantly lower in controls $(7 \pm 4, p<0.001)$.

\subsection{Sleep studies}

On polysomnography, NC and BIISS had similar mean sleep latencies to NREM2 $(6.7 \pm 4.7$ and $9 \pm 4.9 \mathrm{~min}$, respectively, $p=0.14$ ) and REM sleep ( $44 \pm 86$ and $82.6 \pm 48.3 \mathrm{~min}$, respectively, $p=0.09$ ), but sleep efficiency was significantly lower in NC than in BIISS ( $86.8 \pm 11.9 \%$ vs. $94.8 \pm 4.7 \%, p=0.008)$; other polysomnographic data are presented in Table 2 .

In BIISS patients, the actigraphy-based measure of sleep duration was $7 \mathrm{~h} 31 \mathrm{~min} \pm 54 \mathrm{~min}$, and $8 \mathrm{~h} 32 \mathrm{~min} \pm 1 \mathrm{~h} 17 \mathrm{~min}$ after sleep extension $(p=0.02)$.

On MSLT, mean sleep latencies to NREM1 were significantly shorter in NC $(1.8 \pm 1.5 \mathrm{~min})$ compared to BIISS $(4.7 \pm 2.1 \mathrm{~min}$, $p<0.001$ ) and controls (11.4 $\pm 3.3 \mathrm{~min}, p<0.001$ ) (Fig. 1). A mean sleep latency $\leqslant 2.5 \mathrm{~min}$ was found in $16 / 20(80 \%)$ NC patients compared to $3 / 20(15 \%)$ BIISS patients $(p<0.001)$. Mean sleep latencies to NREM2 were similar in NC and BIISS $(8.6 \pm 4.7$ and $8.1 \pm 2.7 \mathrm{~min}$, respectively, $p=0.64)$, but significantly shorter than in controls $(13.4 \pm 3.3 \mathrm{~min}, p<0.001)$. When measuring sleep latencies to either NREM2 or REM (e.g., the sum of the sleep latency to NREM1 and the duration of the first NREM1 sleep sequence), however, NC revealed significantly shorter latencies than BIISS ( $4.4 \pm 2.9$ and $7.9 \pm 3.5 \mathrm{~min}$, respectively, $p<0.001$ ).

\subsection{Subanalysis of MSLT naps with SOREM periods}

SOREM periods occurred in $62 / 85$ naps $(73 \%)$ in NC patients, compared to $13 / 89$ naps $(15 \%)$ in BIISS patients $(p<0.001)$. SOREM periods were not observed in controls. Mean sleep latency below $8 \mathrm{~min}$ was found in each NC patient, but only $17 \mathrm{NC}$ patients $(85 \%)$ had $\geqslant 2$ SOREM periods. Three BIISS patients (15\%) had a mean sleep latency $<8 \mathrm{~min}$ and $\geqslant 2$ SOREM periods. When referring only to naps with SOREM periods, mean sleep latency from lights off to NREM1 did not differ between NC and BIISS $(1.9 \pm 2.3$ and $1.8 \pm 1.3 \mathrm{~min}$, respectively; $p=0.96$ ), but mean sleep latency from lights off to REM sleep was significantly shorter in NC than in BIISS

Table 1

Demographic and clinical characteristics of patients with narcolepsy and cataplexy (NC) and behaviourally induced insufficient sleep syndrome (BIISS), and normal controls.

\begin{tabular}{lllll}
\hline & NC & BIISS & Controls & $p$ \\
\hline Age (mean \pm SD) [years] & $39 \pm 18$ & $36 \pm 13$ & $40 \pm 15$ & 0.78 \\
Gender (men/women) & $11 / 9$ & $10 / 10$ & $11 / 9$ & 0.94 \\
BMI (mean \pm SD) [kg] & $25 \pm 5$ & $24 \pm 5$ & $23 \pm 4$ & 0.14 \\
\hline
\end{tabular}

BMI: body mass index; SD: standard deviation. 
Table 2

Polysomnographic data.

\begin{tabular}{lcccc}
\hline & NC & BIISS & Controls & $p^{\mathrm{a}}$ \\
\hline Total sleep time [min] & $325 \pm 54$ & $406 \pm 26$ & $357 \pm 50$ & 0.03 \\
Sleep efficiency [\%] & $87 \pm 12$ & $95 \pm 5$ & $91 \pm 6$ & 0.013 \\
Sleep latency to NREM2 & $7 \pm 5$ & $9 \pm 5$ & $50 \pm 49$ & $<0.001$ \\
REM sleep latency & $44 \pm 86$ & $83 \pm 48$ & $98 \pm 39$ & 0.02 \\
NREM1 [\%] & $17 \pm 10$ & $7 \pm 7$ & $9 \pm 7$ & 0.001 \\
NREM2 [\%] & $37 \pm 11$ & $51 \pm 9$ & $49 \pm 9$ & $<0.001$ \\
SWS ${ }^{\mathrm{b}}$ [\%] & $13 \pm 7$ & $17 \pm 5$ & $16 \pm 6$ & 0.11 \\
REM [\%] & $20 \pm 9$ & $19 \pm 5$ & $16 \pm 5$ & 0.14 \\
\hline
\end{tabular}

a One-way ANOVA.

b Slow wave sleep (NREM3 + NREM4).

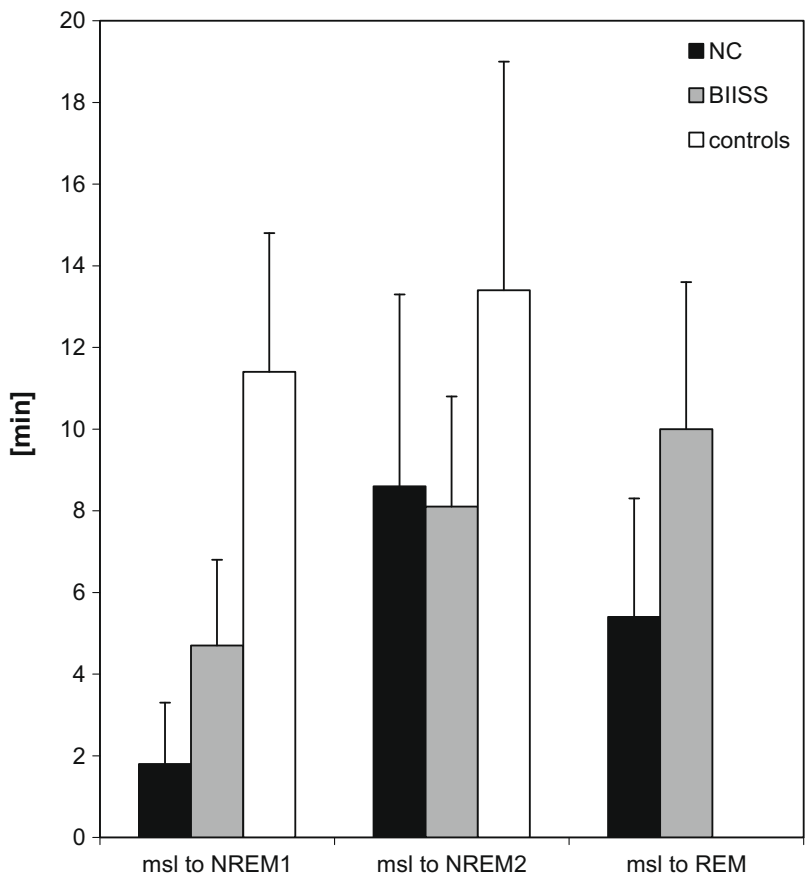

Fig. 1. Mean sleep latencies to NREM1, NREM2, and REM sleep in NC, BIISS, and controls (including standard deviations). msl, Mean sleep latency.

Table 3

MSLT: mean sleep latencies (in minutes) to NREM1 in NC and BIISS.

\begin{tabular}{lll}
\hline & $n$ (naps) & Mean sleep latency to NREM1 \\
\hline NC with SOREM & 62 & $1.9 \pm 2.3$ \\
NC without SOREM & 23 & $1.8 \pm 1.3$ \\
BIISS with SOREM & 13 & $1.8 \pm 1.3$ \\
BIISS without SOREM & 76 & $5.2 \pm 3.4$ \\
\hline
\end{tabular}

$n=$ number of naps.

(5.4 \pm 3.8 and $10.0 \pm 4.0 \mathrm{~min}$, respectively; $p<0.001)$. Conversely, mean sleep latency from lights off to NREM2 was significantly shorter in BIISS than in NC $(6.5 \pm 4.4$ and $10.3 \pm 6.4 \mathrm{~min}$, respectively; $p<0.001$ ). In NC, no difference was found when comparing mean sleep latencies to NREM1 in naps with and without SOREM periods ( $1.9 \pm 2.3$ and $1.8 \pm 1.3 \mathrm{~min}$, respectively; $p=0.85$ ). In BIISS patients, on the other hand, mean sleep latency to NREM1 was significantly shorter in naps with than without SOREM periods $(1.8 \pm 1.3$ and $5.2 \pm 3.4$ min, respectively; $p<0.001)$ (Table 3$)$. In NC, we found no association between number of SOREMs and mean sleep latencies.

\subsection{Sleep stage sequencing in MSLT naps with SOREMs}

In NC, REM sleep occurred immediately after NREM1 in 44/62 (71\%) naps, and was followed in 20/44 (45\%) naps by NREM2; in 24/44 (55\%) naps NREM2 was not achieved. In the remaining 18/ $62(29 \%)$ naps the sequence NREM1-NREM2-REM was observed $(p<0.001)$. In BIISS, REM sleep immediately after NREM1 was found in only $2 / 13$ (15\%) naps, followed by NREM2 in one nap; in $11 / 13$ (85\%) naps the sequence NREM1-NREM2-REM was observed $(p<0.001)$ (Fig. 2). Consecutively, NC had a significantly longer NREM1-NREM2 latency than BIISS $(6.1 \pm 5.8$ and $3.5 \pm 2.5 \mathrm{~min}$, respectively; $p=0.001$ ). However, when referring only to naps without REM prior to NREM2, no difference in NREM1-NREM2 latency was found between NC and BIISS $(2.4 \pm 1.9$ and $3.6 \pm 2.1 \mathrm{~min} ; p=0.14)$.

\section{Discussion}

In our study, we found (1) shorter sleep latencies in MSLT to NREM1 and REM in NC compared to BIISS, (2) but similar sleep latencies to NREM2 in both disorders, which is explained by the observation that (3) the sequence NREM1-REM-NREM2 is more prevalent in NC (71\% of all naps with REM sleep) compared to BIISS, with NREM1-NREM2-REM as the most common sequence (85\%).

In general, sleepiness (as assessed with mean sleep latencies to NREM1) and REM sleep propensity (as assessed with the number of SOREM periods and the latency to REM sleep) were higher in NC than in BIISS patients. However, considering only naps with SOREM periods, mean sleep latencies to NREM1 were similar between the two conditions. In other words, BIISS patients with SOREM periods had significantly shorter mean sleep latencies to NREM1 than BIISS patients without SOREM periods, which is in line with other studies [13]. This finding suggests that the presence of SOREM periods in BIISS is associated with a higher sleep pressure, leading to sleep latencies not discernible from narcoleptic patients. In NC, however, the occurrence of SOREM periods was not associated with shorter sleep latencies on MSLT naps. Furthermore, we could not confirm previous findings of a significant correlation between a higher number of SOREM periods and lower mean sleep latencies in NC [14].

The combination of $\geqslant 2$ SOREM periods and short mean sleep latency on MSLT has been shown to have limited sensitivity and specificity for the diagnosis of NC [2,15]. Indeed, our finding of

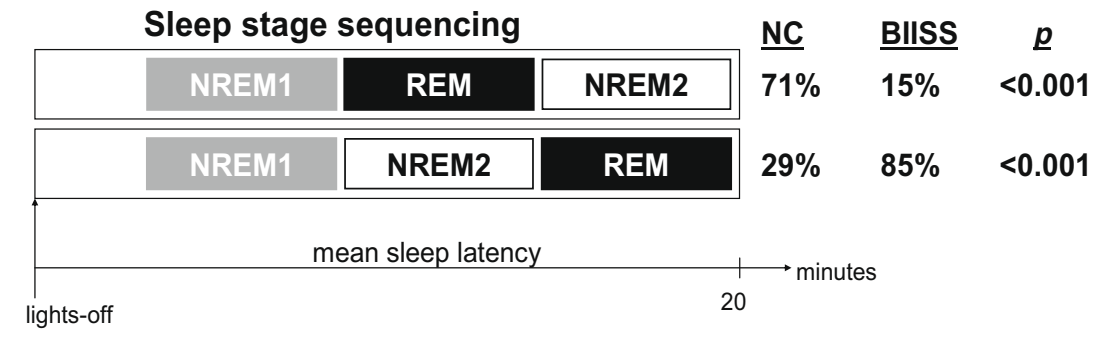

Fig. 2. Different sleep stage sequencing in NC and BIISS patients in MSLT naps with SOREMs (NC: $n=62 / 85$ naps, BIISS: $n=13 / 89$ naps). 
$\leqslant 1$ SOREM period in $15 \%$ of NC patients corroborates the assumption of suboptimal sensitivity of this particular test. On the other hand, regarding specificity, $\geqslant 2$ SOREM periods have also been reported in other sleep-wake disturbances and even in healthy subjects. Chervin et al. found $\geqslant 2$ SOREM periods in almost $5 \%$ of 1145 consecutive patients with suspected or confirmed obstructive sleep apnea [16]. Aldrich et al. reported a frequency of $\geqslant 2$ SOREM periods in $7 \%$ of 1251 patients with sleep-related breathing disorders [2]. Also, Guilleminault et al. observed multiple SOREM periods in patients suffering from upper airway resistance syndrome [15]. Furthermore, $\geqslant 2$ SOREM periods have been observed in a high number of healthy controls. Bishop et al. observed a surprisingly high frequency (17\%) of $\geqslant 2$ SOREM periods among 139 young, drug-free subjects without medical, psychiatric or sleep-related complaints [17]. However, as the same authors acknowledge, no actigraphic recordings were performed in these subjects to confirm regular sleep schedules and to exclude BIISS as a potential explanation of the high frequency of multiple SOREM periods. Similarly, Mignot et al. found multiple SOREM periods in $13.1 \%$ of males and $5.6 \%$ of females of the community-based Wisconsin Sleep Cohort Study [4]. Conversely, in our healthy controls, in whom BIISS was excluded by actigraphic recordings and sleep logs, we could not find any SOREM periods on MSLT. Other groups also failed to demonstrate multiple SOREM periods in healthy controls [18-21]. The reason for these discrepant findings is not clear at the moment and will warrant further studies, as we believe (based on our daily clinical work) that BIISS in the healthy population is frequent.

Until now, there are no systematic studies on the frequency of multiple SOREM periods in BIISS patients. In 1994, Rosenthal first suggested that $\geqslant 2$ SOREM periods in patients without clinical symptomatology for NC might reflect chronic sleep deprivation [22]. We found a combination of $\geqslant 2$ SOREM periods and a mean sleep latency $\leqslant 2.5 \mathrm{~min}$ in $15 \%$ of BIISS patients. This finding ultimately underscores the low specificity and limited reliability of the MSLT in differentiating NC from BIISS, indicating that the clinical interview remains most important in the diagnosis of these sleep-wake disorders. Furthermore, sleep logs, actigraphy, and nocturnal polysomnography are helpful in the distinction of the two disorders.

Sleep stage sequencing is different in NC and BIISS, with NREM1-REM-NREM2 being the most frequent pattern in NC, contrary to NREM1-NREM2-REM in BIISS. The finding of frequent REM prior to NREM2 in NC may primarily indicate that REM sleep regulation is altered in NC. In this line, it has been hypothesized that REM sleep propensity is enhanced in NC, consecutively leading to phenomena which are believed to be fragments of REM sleep (atonia, dream-like states) [23]. Recently, Lu and colleagues [24] identified REM-off neurons in the brainstem, which are densely innervated by the hypocretin neurons. A loss of hypocretin neurons, therefore, would weaken the REM-off side of a REM-on-off flip-flop mechanism, giving way to enhanced REM pressure and finally to REM sleep periods occurring prior to the constitution of NREM2 sleep [25]. However, our finding also might indicate that homeostatic NREM sleep regulation is altered in NC. In fact, little is known about NREM sleep regulation in NC. A polysomnography study suggested that NREM sleep intensity is insufficient in NC, with frequent interruptions and an impaired build-up of slowwake activity during nocturnal NREM sleep [26]. Thus, in accordance with earlier reports, the frequent observation of SOREM episodes with the sequence NREM1-REM-NREM2 might be explained by a dysregulation involving both REM and NREM sleep in NC [14]. Although BIISS patients also often present with increased REM sleep pressure and with SOREM periods, this abnormal NREMREM sleep interaction does not appear to be present in BIISS.
The main limitation of this study is the small sample size. However, our three groups have been strictly matched for age, gender, and BMI. In addition, our study only analyzed narcolepsy patients with clear-cut cataplexy. Hence, our findings cannot be generalized to narcolepsy patients without cataplexy, although this subgroup particularly needs objective features that allow differentiating it from BIISS. Therefore, future studies will want to prospectively compare MSLT findings between BIISS patients and narcolepsy patients with and without cataplexy. Furthermore, we included two NC patients who were not free from drugs affecting the central nervous system (modafinil and sodium valproate, respectively). However, the clinical, polysomnographic and MSLT findings of these two patients did not significantly differ from the remaining NC patients, nor did the exclusion of these two patients alter the results of this study.

In conclusion, we aimed at analyzing differences of MSLT characteristics between NC and BIISS, two conditions whose clinical presentation may show substantial overlap. The need of objective parameters to differentiate between the two conditions is further reflected by the almost equal degree of subjective EDS (estimated by the ESS) in our study population. Nevertheless, the diagnosis of NC cannot be made by means of the MSLT alone but still mostly relies on a thorough clinical interview, together with sleep logs, actigraphy, and nocturnal polysomnography. In cases with similar clinical presentation, however, MSLT results can additionally help differentiating between NC and BIISS. We found that several findings might serve as an indicator for NC and against BIISS: mean sleep latencies to NREM $1 \leqslant 2.5 \mathrm{~min}$, the presence of multiple SOREM periods, and the sequence NREM1-REM-NREM2 may be the best discriminating factors.

\section{References}

[1] Mitler MM, Van den Hoed J, Carskadon MA, Richardson G, Guilleminault C, Dement WC. REM sleep episodes during the MSLT in narcoleptic patients. Electroenceph Clin Neurophysiol 1979;46:479-81.

[2] Aldrich MS, Chervin RD, Malow BA. Value of the multiple sleep latency test (MSLT) for the diagnosis of narcolepsy. Sleep 1997;20:620-9.

[3] American Sleep Disorders Association: International Classification of Sleep Disorders: Diagnostic and Coding Manual. Revised 2005

[4] Mignot E, Lin L, Finn L, Lopes C, Pluff K, Sundstrom ML, et al. Correlates of sleep-onset REM periods during the Multiple Sleep Latency Test in community adults. Brain 2006;129:1609-23.

[5] Ferrara M, De Gennaro L. How much sleep do we need? Sleep Med Rev 2001;5:155-79.

[6] Dinges DF, Rogers NL, Baynard MD. Chronic sleep deprivation. In: Kryger MH, Roth T, Dement WC, editors. Principles and practice of sleep medicine. 4th ed. Philadelphia: 2005.

[7] Sturzenegger C, Bassetti CL. The clinical spectrum of NC: a reappraisal. J Sleep Res 2004;13:395-406.

[8] Zorick F, Roehrs T, Koshorek G, et al. Patterns of sleepiness in various disorders of excessive daytime sleepiness. Sleep 1982;5:S165-174.

[9] Spiegel K, Leproult R, Van Cauter E. Impact of sleep debt on metabolic and endocrine function. Lancet 1999;354:1435-9.

[10] Webb WB, Agnew Jr HW. Sleep: effects of restricted regime. Science 1965;150:1745-7.

[11] Littner MR, Kushida C, Wise M, et al. Standards of practice committee of the American Academy of Sleep Medicine. Practice parameters for clinical use of the multiple sleep latency test and the maintenance of wakefulness test. Sleep 2005;28:113-21.

[12] Rechtschaffen A, Kales A, editors. A manual of standardized terminology, techniques and scoring system for sleep stages of human subjects. Los Angeles: UCLA Brain Information Service/Brain Research Institute; 1968.

[13] Dinges DF, Pack F, Williams K, et al. Cumulative sleepiness, mood disturbances, and psychomotor vigilance performance decrements during a week of sleep restricted to 4-5 hours per night. Sleep 1997;20:267-77.

[14] Tafti M, Villemin E, Carlander B, Besset A, Billiard M. Sleep onset rapid-eyemovement episodes in narcolepsy: REM sleep pressure or nonREM-REM sleep dysregulation? J Sleep Res 1992;1:245-50.

[15] Guilleminault C, Mignot E, Partinen M. Controversies in the diagnosis of narcolepsy. Sleep 1994;17:S1-6.

[16] Chervin RD, Aldrich MS. Sleep onset REM periods during multiple sleep latency tests in patients evaluated for sleep apnea. Am J Respir Crit Care Med 2000;161:426-31. 
[17] Bishop C, Rosenthal L, Helmus T, Roehrs T, Roth T. The frequency of multiple sleep onset REM periods among subjects with no excessive daytime sleepiness. Sleep 1996;19:727-30.

[18] Folkerts M, Rosenthal L, Roehrs $T$, et al. The reliability of the diagnostic features in patients with narcolepsy. Biol Psychiatry 1996;40:208-14.

[19] Richardson GS, Carskadon MA, Flagg W, Van den Hoed J, Dement WC, Mitler MM. Excessive daytime sleepiness in man: multiple sleep latency measurement in narcoleptic and control subjects. Electroencephalogr Clin Neurophysiol 1978;45:621-7.

[20] Mitler MM. The multiple sleep latency test as an evaluation for excessive daytime sleepiness. In: Guilleminault C, editor. Sleeping and waking disorders: indications and techniques. Menlo Park, CA: Addison-Wesley; 1982. p. 145-53.
[21] Amira SA, Johnson TS, Logowitz NB. Diagnosis of narcolepsy using the multiple sleep latency test: analysis of current laboratory criteria. Sleep 1985;8:325-31.

[22] Rosenthal L, Folkerts M, Roehrs T, Zorick F, Roth T. Sleepiness and sleep onse REM periods in the absence of clinical symptomatology. Biol Psychiatry 1994;36:341-3.

[23] Baumann CR, Bassetti CL. Hypocretins (orexins) and sleep-wake disorders. Lancet Neurol 2005;4:673-82.

[24] Lu J, Sherman D, Devor M, Saper CB. A putative flip-flap switch for control of REM sleep. Nature 2006;441:589.

[25] Saper CB, Scammell TE, Lu J. Hypothalamic regulation of sleep and circadian rhythms. Nature 2005;437:1257-63.

[26] Khatami R, Landolt HP, Achermann P, Rétey JV, Werth E, Mathis J, et al. Insufficient non-REM sleep intensity in narcolepsy-cataplexy. Sleep 2007;30:980-9. 


\section{BRIEF COMMUNICATION}

\section{References}

1. Abbott NJ. Evidence for bulk flow of brain interstitial fluid: significance for physiology and pathology. Neurochem Int 2004; $45: 545-552$.

2. Davson H. The Physiology and Pathophysiology of the Cerebral Spinal Fluid. New York, NY: Churchill Livingstone; 1987.

3. Toczylowska B, Chalimoniuk M, Wodowska M, MayznerZawadzk E. Changes in concentration of cerebrospinal fluid components in patients with traumatic brain injury. Brain Res 2006;1104:183-189.

4. Otto F, Gortz P, Fleischer W, Siebler M. Cryopreserved rat cortical cells develop functional neuronal networks on microelectrode arrays. J Neurosci Methods 2003;128:173-181.

5. Ban J, Bonifazi P, Pinato G, et al. Embryonic stem cell-derived neurons form functional networks in vitro. Stem Cells 2007; 25:738-749.

6. Illes S, Fleischer W, Siebler M, et al. Development and pharmacological modulation of embryonic stem cell-derived neuronal network activity. Exp Neurol 2007;207:171-176.

7. Bain G, Kitchens D, Yao M, et al. Embryonic stem cells express neuronal properties in vitro. Dev Biol 1995;168: 342-357.

8. Barberi T, Klivenyi P, Calingasan NY, et al. Neural subtype specification of fertilization and nuclear transfer embryonic stem cells and application in parkinsonian mice. Nat Biotechnol 2003;21:1200-1207.

9. Kim JH, Auerbach JM, Rodriguez-Gomez JA, et al. Dopamine neurons derived from embryonic stem cells function in an animal model of Parkinson's disease. Nature 2002;418: $50-56$.

10. Dihne M, Bernreuther C, Hagel C, et al. Embryonic stem cellderived neuronally committed precursor cells with reduced teratoma formation after transplantation into the lesioned adult mouse brain. Stem Cells 2006;24:1458-1466.

11. Wagenaar DA, Madhavan R, Pine J, Potter SM. Controlling bursting in cortical cultures with closed-loop multi-electrode stimulation. J Neurosci 2005;25:680-688.

12. Werner C, Engelhard K. Pathophysiology of traumatic brain injury. Br J Anaesth 2007;99:4-9.

13. Nilsson OG, Polito A, Saveland H, et al. Are primary supratentorial intracerebral hemorrhages surrounded by a biochemical penumbra? A microdialysis study. Neurosurgery 2006;59: 521-528.

14. Chiang MF, Chiu WT, Lin FJ, et al. Multiparametric analysis of cerebral substrates and nitric oxide delivery in cerebrospinal fluid in patients with intracerebral haemorrhage: correlation with hemodynamics and outcome. Acta Neurochir (Wien) 2006;148:615-621; discussion 621.

15. Danysz W, Parsons CG. Glycine and N-methyl-D-aspartate receptors: physiological significance and possible therapeutic applications. Pharmacol Rev 1998;50:597-664.

16. Johnson MA, Weick JP, Pearce RA, Zhang SC. Functional neural development from human embryonic stem cells: accelerated synaptic activity via astrocyte coculture. J Neurosci 2007; 27:3069-3077.

17. Roy NS, Cleren C, Singh SK, et al. Functional engraftment of human ES cell-derived dopaminergic neurons enriched by coculture with telomerase-immortalized midbrain astrocytes. Nat Med 2006;12:1259-1268.

18. Takahashi K, Tanabe K, Ohnuki M, et al. Induction of pluripotent stem cells from adult human fibroblasts by defined factors. Cell 2007;131:861-872.

19. Yu J, Vodyanik MA, Smuga-Otto K, et al. Induced pluripotent stem cell lines derived from human somatic cells. Science 2007; 318:1917-1920.
20. Watanabe K, Kamiya D, Nishiyama A, et al. Directed differentiation of telencephalic precursors from embryonic stem cells. Nat Neurosci 2005;8:288-296.

21. Nat R, Nilbratt M, Narkilahti S, et al. Neurogenic neuroepithelial and radial glial cells generated from six human embryonic stem cell lines in serum-free suspension and adherent cultures. Glia 2007;55:385-399.

22. Ying QL, Stavridis M, Griffiths D, et al. Conversion of embryonic stem cells into neuroectodermal precursors in adherent monoculture. Nat Biotechnol 2003;21:183-186.

\section{Loss of Hypocretin (Orexin) Neurons With Traumatic Brain Injury}

Christian R. Baumann, MD, ${ }^{1,2}$ Claudio L. Bassetti, MD, ${ }^{1}$ Philipp O. Valko, MD, ${ }^{1}$ Johannes Haybaeck, MD, ${ }^{3}$ Morten Keller, MD, ${ }^{4}$ Erika Clark, ${ }^{2}$ Reto Stocker, MD, ${ }^{5}$ Markus Tolnay, MD, ${ }^{3}$ and Thomas E. Scammell, $\mathrm{MD}^{2}$

Chronic, daytime sleepiness is a major, disabling symptom for many patients with traumatic brain injury (TBI), but thus far, its etiology is not well understood. Extensive loss of the hypothalamic neurons that produce the wake-promoting neuropeptide hypocretin (orexin) causes the severe sleepiness of narcolepsy, and partial loss of these cells may contribute to the sleepiness of Parkinson disease and other disorders. We have found that the number of hypocretin neurons is significantly reduced in patients with severe TBI. This observation highlights the often overlooked hypothalamic injury in TBI and provides new insights into the causes of chronic sleepiness in patients with TBI.

Ann Neurol 2009;66:555-559

Approximately 1.4 million people sustain traumatic brain injury (TBI) each year in the United States. ${ }^{1}$ Coma and obtundation are common during the acute period, but even after 6 months recovery, $43 \%$ of patients have symptoms of sleep disorders. ${ }^{2,3}$ In a pro-

From the ${ }^{1}$ Department of Neurology, University Hospital, Zurich, Switzerland; ${ }^{2}$ Department of Neurology, Beth Israel Deaconess Medical Center, Boston, MA; and ${ }^{3}$ Department of Neuropathology, ${ }^{4}$ Department of Forensic Medicine, and ${ }^{5}$ Department of Surgical Intensive Care, University Hospital, Zurich, Switzerland.

Address correspondence to Dr Baumann, Department of Neurology, University Hospital Zurich, Frauenklinikstrasse 26, $8091 \mathrm{Zu}$ rich, Switzerland. E-mail: christian.baumann@usz.ch

Potential conflict of interest: Nothing to report.

Received Jan 25, 2009, and in revised form Jul 7. Accepted for publication Jul 31, 2009. Published online in Wiley InterScience (www.interscience.wiley.com). DOI: 10.1002/ana.21836 
spective study of 65 TBI patients, 1 in 4 patients reported excessive daytime sleepiness (EDS) irrespective of the location or severity of the head trauma. ${ }^{2}$ Another study of 87 patients revealed similar results, with sleepiness in $25 \%$ after TBI. $^{3}$

Although chronic sleepiness is common after head trauma, the cause remains unclear. Injury to ascending arousal pathways from the brainstem may contribute, but recent findings suggest a key role for the neuropeptides hypocretin-1 and -2 (also known as orexin- $\mathrm{A}$ and -B). ${ }^{2,4,5}$ Hypocretins are made by a small cluster of neurons in the lateral hypothalamus that play an essential role in promoting wakefulness. Selective loss of the hypocretin-producing neurons causes sleepiness and cataplexy in $>90 \%$ of patients with narcolepsy, ${ }^{5-9}$ and identical symptoms occur in mice genetically lacking hypocretins. ${ }^{10}$ Injury to the hypothalamus is common in patients with TBI, ${ }^{11}$ but its clinical impact remains unclear. We recently found that TBI patients have a marked reduction in cerebrospinal fluid (CSF) hypocretin-1 levels in the first days after injury. ${ }^{4}$ Even after 6 months recovery, many TBI patients with EDS have persistently low hypocretin-1 levels. ${ }^{2}$ Thus, we hypothesized that post-traumatic sleepiness is caused, at least in part, by injury to the wake-promoting hypocretin neurons.

\section{Materials and Methods \\ Subjects}

We collected brains at autopsy from 4 patients who died 7 to 42 (mean 19) days after severe TBI (Supplemental Table). Causes of death were multiorgan failure $(\mathrm{n}=2)$, elevated intracranial pressure $(\mathrm{n}=1)$, and brain death $(\mathrm{n}=1)$. We obtained 4 additional brains from control subjects with no clinical evidence of TBI or other neurological disorders. Those subjects died from ruptured aortic aneurysm $(\mathrm{n}=1)$, liver failure $(\mathrm{n}=1)$, drowning $(\mathrm{n}=1)$, and myocardial infarction $(n=1)$. First-degree relatives gave written informed consent for autopsy and tissue research. This study was approved by the human subjects committees of Kantonale Ethikkommission Zurich and Beth Israel Deaconess Medical Center.

\section{Tissue Processing}

We fixed whole hypothalami in $10 \%$ buffered formalin and stored them at $4^{\circ} \mathrm{C}$. After the tissue equilibrated for 2 to 3 days in phosphate-buffered saline with $20 \%$ sucrose, $10 \%$ formalin, and $0.1 \%$ diethyl pyrocarbonate $(\mathrm{pH} 7.4)$, we coronally sectioned the right and left halves of the hypothalamus at $40 \mu \mathrm{m}$ in a $1: 24$ series. We stored sections at $-20^{\circ} \mathrm{C}$ in a cryoprotectant solution. ${ }^{\text {? }}$

\section{Immunohistochemistry}

We immunostained sections for hypocretin-1 (orexin A) as described previously. ${ }^{9}$ Briefly, after treatment with $3 \%$ hydrogen peroxide and blocking in 3\% normal horse serum, we incubated sections overnight in goat anti-hypocretin-1 anti- serum (1:5,000; Santa Cruz Biotechnology, Santa Cruz, CA) and then in biotinylated donkey antigoat secondary antiserum at 1:500. We then exposed the sections to avidinbiotin complex and diaminobenzidine to produce light brown labeling of hypocretin-containing neurons. The specificity of the antiserum was tested and confirmed in hypothalamic tissue from hypocretin-knockout mice. ${ }^{12}$

To test whether other neurons in the lateral hypothalamus are lost with TBI, we immunostained adjacent sections for melanin-concentrating hormone $(\mathrm{MCH}$; rabbit primary antiserum, 1:10,000; a kind gift from Eleftheria Maratos-Flier).

Finally, to test whether gliosis accompanies hypocretin neuron loss, we double-labeled sections for hypocretin-1 (1: $5,000)$ and glial fibrillary acidic protein (GFAP), a marker of astrocytes. Hypocretin was labeled using goat antihypocretin-1 antiserum $(1: 5,000)$, biotinylated donkey antigoat immunoglobulin (Ig) G (Jackson Laboratories, Bar Harbor, ME), and Alexa 488-conjugated streptavidin (Invitrogen, Carlsbad, CA). GFAP was labeled using rabbit anti-GFAP primary antiserum (1:2,000; Millipore, Billerica, MA) and Cy3-conjugated donkey antirabbit IgG secondary antiserum (Jackson ImmunoResearch Laboratories, West Grove, PA).

\section{Cell Counts}

After immunostaining, we examined sections at $\times 200$ with a Zeiss Axiophot microscope. We digitally superimposed a counting grid on every section and counted all hypocretinand $\mathrm{MCH}$-immunoreactive neurons across a 1:24 series of the entire hypothalamus. To prevent miscounting, we used a fine-scale counting grid that encompassed no more than 10 neurons per grid sector. Only clearly immunolabeled neurons with neuronal morphology, outgoing axons and dendrites, and a nuclear void were counted. To estimate accurately the numbers of hypocretin and $\mathrm{MCH}$ neurons in each section, we performed Abercrombie corrections based on the size of the cell nuclei in each subject. ${ }^{13}$ We then calculated the total numbers of hypocretin and $\mathrm{MCH}$ neurons by multiplying the cell counts per section by the section sampling frequency (1:24). We also counted GFAP-immunolabeled astrocytes in 30 randomly selected $400 \times 400 \mu \mathrm{m}$ sites within the lateral and posterior hypothalamus. Cell counts were compared between groups using Student $t$ tests.

\section{Results}

In this pilot study, the TBI patients were younger $(51 \pm 19$ years) than the controls $(69 \pm 18$ years $)$, and all subjects were male except for 1 woman in the control group. Postmortem delays were similar in both groups (TBI: $12 \pm 3$ hours; controls: $13 \pm 3$ hours) (Supplemental Table).

The number of hypocretin neurons in TBI subjects was $27 \%$ less than in controls $(23,655 \pm 5,035$ vs $32,318 \pm 3060$ neurons; $p=0.001$ ) (Fig 1). Among the TBI patients, the number of hypocretin neurons differed moderately between the left and right sides of the hypothalamus and between individuals, reflecting the asymmetry and heterogeneity of TBI. Many hypocretin neurons in TBI patients were small and py- 


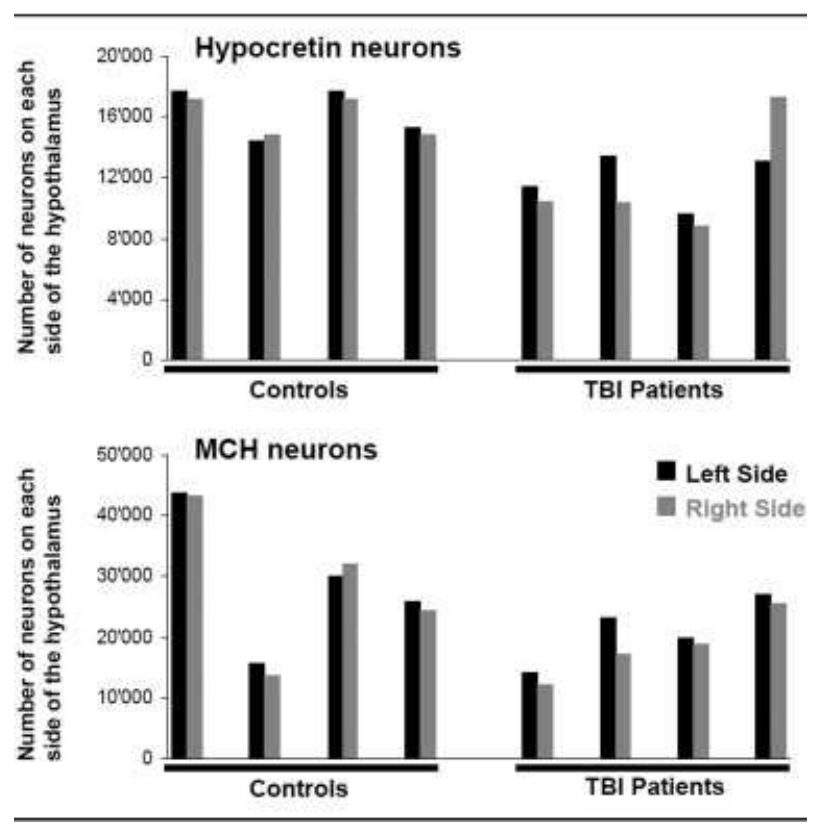

Fig 1. Overview of hypocretin and melanin-concentrating hormone $(M C H)$ cell counts. Patients with traumatic brain injury (TBI) have fewer hypocretin neurons. On average, TBI patients have a total of 23,655 hypocretin neurons, whereas controls have 32,318 neurons ( $\mathrm{p}=0.001$ ). The numbers of hypocretin neurons on the left and right sides of the hypothalamus differ more in TBI patients, probably reflecting asymmetry of the trauma. TBI patients also have fewer MCH neurons than controls $(39,540$ in total vs 57,176; $\mathrm{p}=0.06)$.

knotic, and the density of hypocretin fibers appeared reduced. Although TBI brains contained fewer hypocretin neurons, the neurons were distributed in a normal pattern, thus demonstrating the reliability and completeness of the cell counts (Fig 2). In all subjects, the complete hypocretin neuron field was examined (Supplemental Fig A). In controls, the total number of hypocretin neurons and their distribution across the lateral and posterior hypothalamus were similar to prior reports. $7,8,14,15$

Hypothalami from TBI patients also contained in- tense perivascular hypocretin immunoreactivity (Fig 3). This amorphous staining was present around small vessels in the posterior and lateral hypothalamus but not elsewhere. The perivascular staining may represent phagocytized hypocretin peptide following damage to the hypocretin neurons.

TBI patients also had 31\% fewer $\mathrm{MCH}$ neurons than controls $(39,540 \pm 10,900$ vs $57,176 \pm 24,016)$, but this reduction was not quite statistically significant $(p=0.06)$, probably because of variable numbers of $\mathrm{MCH}$ neurons. If one excludes the female control subject with surprisingly low numbers of $\mathrm{MCH}$ neurons (control subject 2), then the TBI subjects had 29\% fewer hypocretin neurons than controls (33,342 \pm 2,785; $p=0.002$ ) and $40 \%$ fewer $\mathrm{MCH}$ neurons $(66,434 \pm 18,730 ; p=0.003)$.

The density of astrocytes as assessed by GFAP staining was significantly higher $(21 \pm 12$ vs $7 \pm 2$ per $\left.\mathrm{mm}^{2} ; p<0.001\right)$ in TBI patients than in controls (Supplemental Fig B). The pattern of gliosis differed across the TBI patients; double immunofluorescence staining showed that patients with more severe loss of hypocretin neurons had more GFAP-labeled astrocytes $(r=-0.46, p=0.01)$.

\section{Discussion}

In this pilot study, we found that patients with severe TBI have a significant loss of hypocretin-producing neurons. This injury was not limited to the hypocretin neurons, as there was a partial loss of neurons producing $\mathrm{MCH}$ and patchy gliosis throughout the hypothalamus. These findings demonstrate that TBI can injure the hypothalamus and suggest that reduced hypocretin signaling may contribute to the persistent sleepiness often seen in TBI patients.

Descriptions of sub-cortical injury in TBI often focus on the midbrain and subthalamus, ${ }^{16}$ but hypothalamic injury is common. One early study showed damage to the hypothalamus in $42 \%$ of deceased TBI patients. ${ }^{11}$ Other reports describe pituitary and hypothalamic dys-
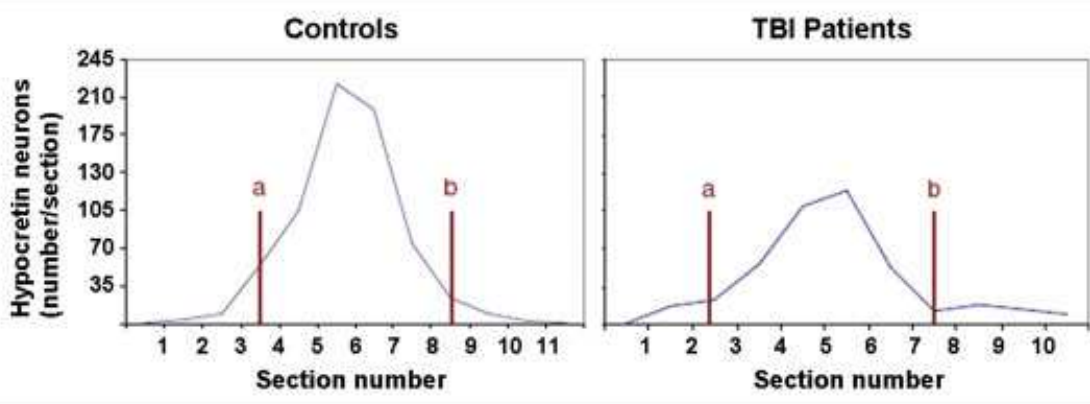

Fig 2. Traumatic brain injury (TBI) patients and controls have similar caudal-rostral distributions of hypocretin neurons. The graphs show the average number of hypocretin neurons per section (mean values across all cases). Overall, TBI patients have fewer neurons, especially in the center of the hypocretin field. Level a: fornix touches the dorsal edge of the mammillary bodies; level b: fornix next to the paraventricular nucleus. [Color figure can be viewed in the online issue, which is available at www.interscience.wiley.com] 


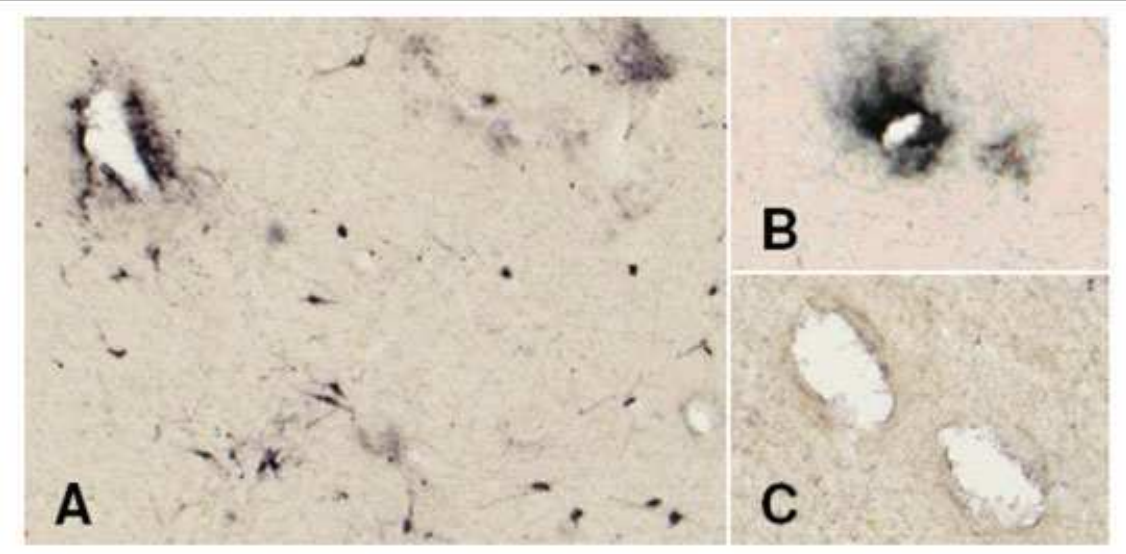

Fig 3. Traumatic brain injury patients have dense perivascular hypocretin immunoreactivity within the hypothalamic hypocretin field $(A, B)$, but not in adjacent areas $(C)$.

function, ${ }^{17,18}$ although to our knowledge, ours is the first to describe injury to specific types of hypothalamic neurons. In our patients, the loss of hypocretin neurons, coupled with gliosis and intense perivascular hypocretinimmunoreactive debris, strongly suggests that severe TBI can directly or indirectly kill hypocretin neurons. Perhaps the hypocretin neurons are injured by the same shearing forces that often damage the midbrain, but vasospasm, or elevated intracranial pressure leading to compression or reduced perfusion of the hypothalamus, may also contribute. ${ }^{11,17,18}$

Chronic excessive daytime sleepiness is a major disabling symptom for many TBI patients, but so far, its etiology is not well understood. ${ }^{2}$ We believe that partial loss of hypocretin neurons may contribute to chronic sleepiness after TBI. Clearly, extensive loss of hypocretin neurons is sufficient to cause the sleepiness of narcolepsy with cataplexy, ${ }^{5-9}$ and some hypothesize that a partial loss of these cells causes the generally milder sleepiness of narcolepsy without cataplexy and Parkinson disease. $^{14,15,19}$ This assumption is supported by our recent finding of reduced CSF hypocretin-1 levels in sleepy TBI patients who survived their injury. ${ }^{2}$ Although the association between lumbar CSF hypocretin levels and the number of intact hypocretin neurons is still poorly understood, we hypothesize that reduced CSF levels of hypocretin in TBI patients may reflect partial loss of the hypocretin neurons and perhaps some reduction in the activity of the surviving cells. However, it is likely that reduced hypocretin signaling is not the only factor to cause sleepiness after TBI. About $\frac{1}{3}$ of TBI patients have brainstem injuries, often near the midbrain tegmentum, that may disrupt ascending monoaminergic or cholinergic wake-promoting pathways. ${ }^{20}$ In addition, some TBI patients have hypersomnolence, with excessively large amounts of sleep each day. Hypersomnolence is common with hypothalamic and rostral brainstem injuries, but is uncommon with selective loss of the hypo- cretin neurons. ${ }^{21}$ Loss of the $\mathrm{MCH}$ neurons may also contribute to the pathophysiology of post-traumatic sleep-wake disturbances, as these cells are hypothesized to regulate rapid eye movement sleep. ${ }^{22,23}$ Overall, it seems likely that the persistent sleepiness in some TBI patients is due to a combination of reduced hypocretin signaling and injury to other sleep-wake regulating systems.

This study has some limitations that should be addressed in future research. We examined a small number of brains, and the control subjects were not fully matched. However, our TBI patients had significantly fewer hypocretin neurons, although they were younger than the controls. In addition, we did not examine other wake-promoting systems, and the extent of injury to other arousal pathways should be further investigated in larger human and animal studies. Last, we studied patients with fatal TBI, which probably introduced some selection bias. It is possible that non-fatal TBI is associated with a less severe loss of hypocretin neurons.

TBI often results in chronic sleepiness, and the observation that wake-promoting hypocretin neurons are lost with TBI provides new insights into the underlying pathophysiology that may lead to novel treatment options. For example, stimulants such as modafinil that are helpful in narcolepsy and other hypocretin-deficient disorders should be studied systematically in affected TBI patients. ${ }^{24}$ Even better, hypocretin agonists could become an elegant and tailored treatment option not only for sleepy individuals with narcolepsy but also for those with TBI.

This study was supported by the Foerderungskredit of the University of Zurich, Switzerland (Ch.B.), the Schweizerischer Versicherungsverband (SW), Switzerland (Ch.B.), and National Institutes of Health grant MH062589 (T.S.). 


\section{References}

1. Rutland-Brown W, Langlois JA, Thomas KE, Xi YL. Incidence of traumatic brain injury in the United States, 2003. J Head Trauma Rehabil 2006;21:544-548.

2. Baumann CR, Werth E, Stocker R, et al. Sleep-wake disturbances 6 months after traumatic brain injury: a prospective study. Brain 2007;130:1873-1883.

3. Castriotta RJ, Wilde MC, Lai JM, et al. Prevalence and consequences of sleep disorders in traumatic brain injury. J Clin Sleep Med 2007;3:349-356.

4. Baumann CR, Stocker R, Imhof HG, et al. Hypocretin-1 (orexin A) deficiency in acute traumatic brain injury. Neurology 2005;65:147-149.

5. España RA, Scammell TE. Sleep neurobiology for the clinician. Sleep 2004;27:811-820.

6. Adamantidis AR, Zhang F, Aravanis AM, et al. Neural substrates of awakening probed with optogenetic control of hypocretin neurons. Nature 2007; 450:420-424.

7. Peyron C, Faraco J, Rogers W, et al. A mutation in a case of early onset narcolepsy and a generalized absence of hypocretin peptides in human narcoleptic brains. Nat Med 2000;6:991-997.

8. Thannickal TC, Moore RY, Nienhuis R, et al. Reduced number of hypocretin neurons in human narcolepsy. Neuron 2000;27: 469-474.

9. Crocker A, España RA, Papadopoulou M, et al. Concomitant loss of dynorphin, NARP, and orexin in narcolepsy. Neurology 2005;65:1184-1188.

10. Chemelli RM, Willie JT, Sinton CM, et al. Narcolepsy in orexin knockout mice: molecular genetics of sleep regulation. Cell 1999;98:437-451.

11. Crompton MR. Hypothalamic lesions following closed head injury. Brain 1971;94:165-172.

12. Baumann CR, Clark EL, Pedersen NP, Hecht JL, Scammell TE. Do enteric neurons make hypocretin? Regul Pept 2008;147:1-3.

13. Guillery RW. On counting and counting errors. J Comp Neurol 2002;447:1-7.

14. Fronczek R, Overeem S, Lee SY, et al. Hypocretin (orexin) loss in Parkinson's disease. Brain 2007;130:1577-1585.

15. Thannickal TC, Lai YY, Siegel JM. Hypocretin (orexin) cell loss in Parkinson's disease. Brain 2007;130:1586-1595.

16. Ropper AH, Miller DC. Acute traumatic midbrain hemorrhage. Ann Neurol 1985;18:80-86.

17. Karavitaki N, Wass J, Henderson Slater JD, Wade D. A case of post-traumatic isolated ACTH deficiency with spontaneous recovery 9 months after the event. J Neurol Neurosurg Psychiatry 2006;77:276-277.

18. Schneider HJ, Aimaretti G, Kreitschmann-Andermahr I, et al. Hypopituitarism. Lancet 2007;369:1461-1470.

19. Scammell TE. The frustrating and mostly fruitless search for an autoimmune cause of narcolepsy. Sleep 2006; 29: 601-2.

20. Crompton MR. Brainstem lesions due to closed head injury. Lancet 1971;1:669-673.

21. Scammell TE. Secondary narcolepsy. In: Culebras A, ed. Sleep Disorders and Neurologic Diseases. 2nd ed. New York, NY: Informa Healthcare; 2007:117-134.

22. Hassani OK, Lee MG, Jones BE. Melanin-concentrating hormone neurons discharge in a reciprocal manner to orexin neurons across the sleep-wake cycle. Proc Natl Acad Sci U S A 2009; 106:2418-2422.

23. Adamantidis A, de Lecea L. Physiological arousal: a role for hypothalamic systems. Cell Mol Life Sci 2008;65:1475-1488.

24. Scammell TE. Modafinil: mechanisms of action. In: Bassetti CL, Billiard M, Mignot E, eds. Narcolepsy and Hypersomnia. New York, NY: Informa Healthcare; 2007:547:559.
Brainstem ${ }^{1} \mathrm{H}$ Nuclear Magnetic Resonance (NMR) Spectroscopy: Marker of Demyelination and Repair in Spinal Cord

Aleksandar Denic, MD, ${ }^{1}$ Allan Bieber, PhD, ${ }^{1}$ Arthur Warrington, PhD, ${ }^{1}$ Prasanna K. Mishra, PhD, ${ }^{2}$ Slobodan Macura, $\mathrm{PhD},{ }^{2}$ and Moses Rodriguez, $\mathrm{MD}^{1}$

Measuring in vivo spinal cord injury and repair remains elusive. Using magnetic resonance spectroscopy (MRS) we examined brainstem $\mathrm{N}$-acetyl-aspartate (NAA) as a surrogate for spinal cord injury in two mouse strains with different reparative phenotypes following virus-induced demyelination. Swiss Jim Lambert (SJL) and Friend Virus B (FVB) mice progressively demyelinate with axonal loss. FVB mice demyelinate similarly but eventually remyelinate coincident with functional recovery. Brainstem NAA levels drop in both but recover in FVB mice. Chronically infected SJL mice lost $30.5 \%$ of spinal cord axons compared to FVB mice $(7.3 \%)$. In remyelination-enhancing or axon-preserving clinical trials, brainstem MRS may be a viable endpoint to represent overall spinal cord dysfunction.

Ann Neurol 2009;66:559-564

New, noninvasive technologies to evaluate spinal cord injury and repair are needed. Proton magnetic resonance spectroscopy $\left({ }^{1} \mathrm{H}-\mathrm{MRS}\right)$ is one ideal method; however, because the spinal cord is small and surrounded by bone, direct spectroscopy has been limited. During retrograde labeling studies of demyelinated spinal cord axons, we observed a reduction in neuron cell bodies in the brainstem labeled with fluorescent markers indicating death or dysfunction. ${ }^{1}$ This led to the concept that neuron health measured at the brainstem with MRS may reflect the integrity of many ascending and descending spinal cord pathways. By following virus-induced spinal cord disease in two strains of

From the Departments of ${ }^{1}$ Neurology and ${ }^{2}$ Biochemistry, Mayo Clinic College of Medicine, Rochester, MN.

Address correspondence to Dr Moses Rodriguez, Department of Neurology, Mayo Medical School, Guggenheim 442B, 200 First Street, SW, Rochester, MN 55905. E-mail: rodriguez.moses@mayo.edu

Additional Supporting Information may be found in the online version of this article.

Potential conflict of interest: Nothing to report.

Received Feb 12, 2009, and in revised form Apr 29. Accepted for publication May 18, 2009. Published online in Wiley InterScience (www.interscience.wiley.com). DOI: 10.1002/ana.21758 


\title{
Increase of Histaminergic Tuberomammillary Neurons in Narcolepsy
}

\author{
Philipp O. Valko, MD, ${ }^{1,2}$ Yury V. Gavrilov, MD, PhD, 1,2,3 Mihoko Yamamoto, MS, ${ }^{1}$ \\ Hasini Reddy, MD, DPhil, ${ }^{4}$ Johannes Haybaeck, MD, PhD, ${ }^{5}$ \\ Emmanuel Mignot, MD, PhD, ${ }^{6}$ Christian R. Baumann, MD, ${ }^{1,2}$ and \\ Thomas E. Scammell, MD ${ }^{1}$
}

Objective: Narcolepsy is caused by loss of the hypothalamic neurons producing the orexin/hypocretin neuropeptides. One key target of the orexin system is the histaminergic neurons of the tuberomammillary nucleus (TMN), an essential wake-promoting system. As cerebrospinal fluid histamine levels may be low in patients with narcolepsy, we examined histaminergic neurons in patients with narcolepsy and in 2 mouse models of narcolepsy.

Methods: We counted the number of hypothalamic neurons producing orexin, melanin-concentrating hormone, and histamine in 7 narcolepsy patients and 12 control subjects using stereological techniques. We identified histaminergic neurons using immunostaining for histidine decarboxylase. We also examined these systems in 6 wild-type mice, 6 orexin/ataxin-3 transgenic mice, and 5 orexin ligand knockout mice.

Results: Compared to controls, narcolepsy patients had 94\% more histaminergic TMN neurons $(233,572 \pm 49,476$ vs $120,455 \pm 10,665, p<0.001)$. This increase was higher in 5 narcolepsy patients with $>90 \%$ orexin neuron loss than in 2 patients with $\leq 75 \%$ orexin neuron loss $(252,279 \pm 46,264$ vs $186,804 \pm 1,256, p=0.03)$. Similarly, the number of histaminergic TMN neurons was increased $53 \%$ in orexin ligand knockout mice compared to wild-type mice, whereas orexin/ataxin-3 transgenic mice showed an intermediate $28 \%$ increase.

Interpretation: This surprising increase in histaminergic neurons in narcolepsy may be a compensatory response to loss of excitatory drive from the orexin neurons and may contribute to some of the symptoms of narcolepsy such as preserved consciousness during cataplexy and fragmented nighttime sleep. In addition, this finding may have therapeutic implications, as medications that enhance histamine signaling are now under development.

ANN NEUROL 2013;74:794-804

N arcolepsy is a common cause of chronic sleepiness that is often accompanied by distinctive symptoms including cataplexy, brief episodes of muscle weakness triggered by strong emotions. Although narcolepsy has been recognized by clinicians for $>100$ years, the underlying cause was a complete mystery until 13 years ago, when 2 research groups discovered that narcolepsy is caused by a loss of the hypothalamic neurons producing the orexin (hypocretin) neuropeptides. ${ }^{1-3}$ These observa- tions substantially advanced the field, but the cause and consequences of orexin neuron loss remain unknown.

Among the targets of the orexin neurons, the histaminergic system plays an especially important role in promoting wakefulness. ${ }^{4}$ The tuberomammillary nucleus (TMN) in the posterior hypothalamus is the sole neuronal source of histamine, and the orexin neurons heavily innervate and excite the TMN neurons. ${ }^{5-8}$ Like other monoaminergic systems, the histaminergic neurons are

View this article online at wileyonlinelibrary.com. DOI: 10.1002/ana.24019

Received Sep 30, 2012, and in revised form Jul 12, 2013. Accepted for publication Aug 10, 2013.

Address correspondence to Dr Scammell, Department of Neurology, Beth Israel Deaconess Medical Center, 330 Brookline Avenue, Boston, MA 02215. E-mail: tscammel@bidmc.harvard.edu

From the ${ }^{1}$ Department of Neurology, Beth Israel Deaconess Medical Center, Boston, MA; ${ }^{2}$ Department of Neurology, University Hospital of Zurich, Zurich, Switzerland; ${ }^{3}$ Department of General Pathology and Pathological Physiology, Institute of Experimental Medicine, St Petersburg, Russia; ${ }^{4}$ Department of Neuropathology, Beth Israel Deaconess Medical Center, Boston, MA; ${ }^{5}$ Department of Neuropathology, Institute of Pathology, Medical University of Graz, Graz, Austria; and 'Department of Psychiatry, Stanford University Center for Narcolepsy, Palo Alto, CA. 
most active during wakefulness, ${ }^{9-11}$ and they are necessary for generating normal wakefulness, as histamine antagonists are sedating in humans and other species. ${ }^{12,13}$ In addition, the histaminergic system appears essential for the wake-promoting effects of orexins, as mice lacking histamine $\mathrm{H} 1$ receptors do not show an arousal response to orexin. ${ }^{14}$ Furthermore, in mice globally lacking orexin receptor- 2 signaling, focal restoration of this receptor in the TMN region rescues sleepiness by reestablishing the normal excitatory signals from the orexin neurons. ${ }^{8}$ Measuring histamine tone in humans is challenging, but as predicted from these observations, histamine levels in lumbar cerebrospinal fluid (CSF) are low in many patients with narcolepsy. ${ }^{15-17}$ Collectively, these observations demonstrate the importance of the histaminergic system in the neurobiology of narcolepsy, but it remains unknown if the reduction in histamine signaling is caused by injury to the histaminergic neurons or reduced activity in TMN neurons.

To assess these possibilities, we examined the histaminergic neurons in the brains of narcoleptic and control subjects. We hypothesized that the number of histaminergic neurons would be normal or possibly reduced if the neuropathological process that kills the orexin neurons also affects or injures the histaminergic neurons. We immunostained hypothalamic sections for histidine decarboxylase (HDC), the key enzyme for synthesizing histamine, ${ }^{18}$ and counted neurons using unbiased stereological techniques. To further examine this phenomenon, we then repeated these experiments in 2 mouse models of narcolepsy.

\section{Materials and Methods}

\section{Human Subjects}

We studied hypothalamic tissue from 7 patients with narcolepsy with cataplexy (NC) and from 12 control subjects with no clinical evidence of neurological or psychiatric disease (Table). Control brains were provided by the Neuropathology Department of Beth Israel Deaconess Medical Center $(n=7)$ and by the Pathology Institute of the Medical University of Graz, Austria $(\mathrm{n}=5)$. Brains from NC patients were provided by Stanford University (Subjects A, B, F), Beth Israel Deaconess Medical Center (Subjects C, D, E), and Massachusetts General Hospital (Subject G). Brain pH at the time of autopsy was not measured. The local ethics committees of all involved institutes approved the study protocol.

All NC patients had a history of severe daytime sleepiness and cataplexy for several decades. In Subject G, cataplexy had decreased in frequency over the duration of his illness and was rare during the 7 years prior to death. Subject $\mathrm{C}$ had Alzheimer disease for the last 8 years of his life. We reported orexin neuron counts from Subjects A, B, and C in prior studies. ${ }^{19,20}$

The mean age was similar in the control and NC groups $(67.4 \pm 13.6$ years vs $69.3 \pm 13.1$ years, respectively, $p=0.77)$.
Seven of the 12 controls were male, and all NC patients were male $(p=0.07)$. All NC patients and 10 controls were Caucasians; 2 controls were African American (Subjects 5 and 7). Age, postmortem delay, and orexin A, melanin-concentrating hormone $(\mathrm{MCH})$, and HDC cell counts did not differ between male and female controls. The numbers of neurons expressing orexin $\mathrm{A}, \mathrm{MCH}$, or HDC in the control subjects did not correlate with age, fixation time, or postmortem delay.

\section{Mice}

We examined the hypothalami of 6 wild-type (WT) C57BL/6J mice (5 males), 5 prepro-orexin ligand knockout $(\mathrm{KO})$ mice (3 males), and 6 orexin/ataxin-3 (Atx) transgenic mice (4 males). The orexin $\mathrm{KO}$ mice have a neomycin cassette inserted into the prepro-orexin gene and lack the orexin A and B neuropeptides. ${ }^{21,22}$ In Atx mice, the human prepro-orexin promoter drives expression of ataxin-3, a toxic transgene that selectively kills most of the orexin neurons by around 12 weeks of age. ${ }^{23,24}$ Thus, these Atx mice are an excellent model of the severe loss of the orexin-producing neurons that occurs in people with NC and allow one to examine the roles of other neurotransmitters (eg, dynorphin and glutamate) produced in the orexin neurons. ${ }^{19,25,26}$ To maximize genetic homogeneity, we backcrossed the Atx and orexin $\mathrm{KO}$ lines with C57BL/6J mice for 8 to 10 generations. We genotyped mice as described previously. ${ }^{24}$ None of the mice was treated with any medications or used in other experiments. Mice were euthanized at similar ages: WT, $38 \pm 10$ weeks; orexin KO, $36 \pm 17$ weeks; and Atx, $23 \pm 1.3$ weeks $(p=0.14)$. We also used $\mathrm{MCH} \mathrm{KO}$ and HDC KO mice to test the specificity of primary antisera. The Institutional Animal Care and Use Committees of Beth Israel Deaconess Medical Center and Harvard Medical School approved these animal studies.

\section{Human Brain Tissue}

We studied both right and left halves of the hypothalami of all control brains and of 2 NC brains (Subjects C and E), but obtained only the right or left half of the hypothalamus in the remaining $5 \mathrm{NC}$ subjects. Postmortem delay of control brains was $19.8 \pm 7.1$ hours (range $=1-26$ hours). Information on postmortem delay of narcoleptic brains could be obtained in 5 subjects $(11.8 \pm 6.9$ hours, $p=0.07)$. The hypothalami were fixed in $10 \%$ buffered formalin and stored at $4{ }^{\circ} \mathrm{C}$. The fixation times varied from 1 week to 2 years in control brains and from 2 months to 11 years in narcoleptic brains. After equilibration in phosphate-buffered saline (PBS) with $20 \%$ sucrose, $10 \%$ formalin, and $0.1 \%$ diethyl pyrocarbonate (DEPC; $\mathrm{pH} 7.4$ ) for 3 to 4 days, we coronally sectioned the hypothalamic blocks at $40 \mu \mathrm{m}$ on a freezing microtome into a 1:24 series. We stored the sections at $-20^{\circ} \mathrm{C}$ in a cryoprotectant solution (25\% glycerol, 35\% ethylene glycol, and 40\% DEPC-PBS).

\section{Mouse Brain Tissue}

Under deep anesthesia, we perfused mice with saline followed by $10 \%$ formalin. Brains were cryoprotected in PBS with $20 \%$ 


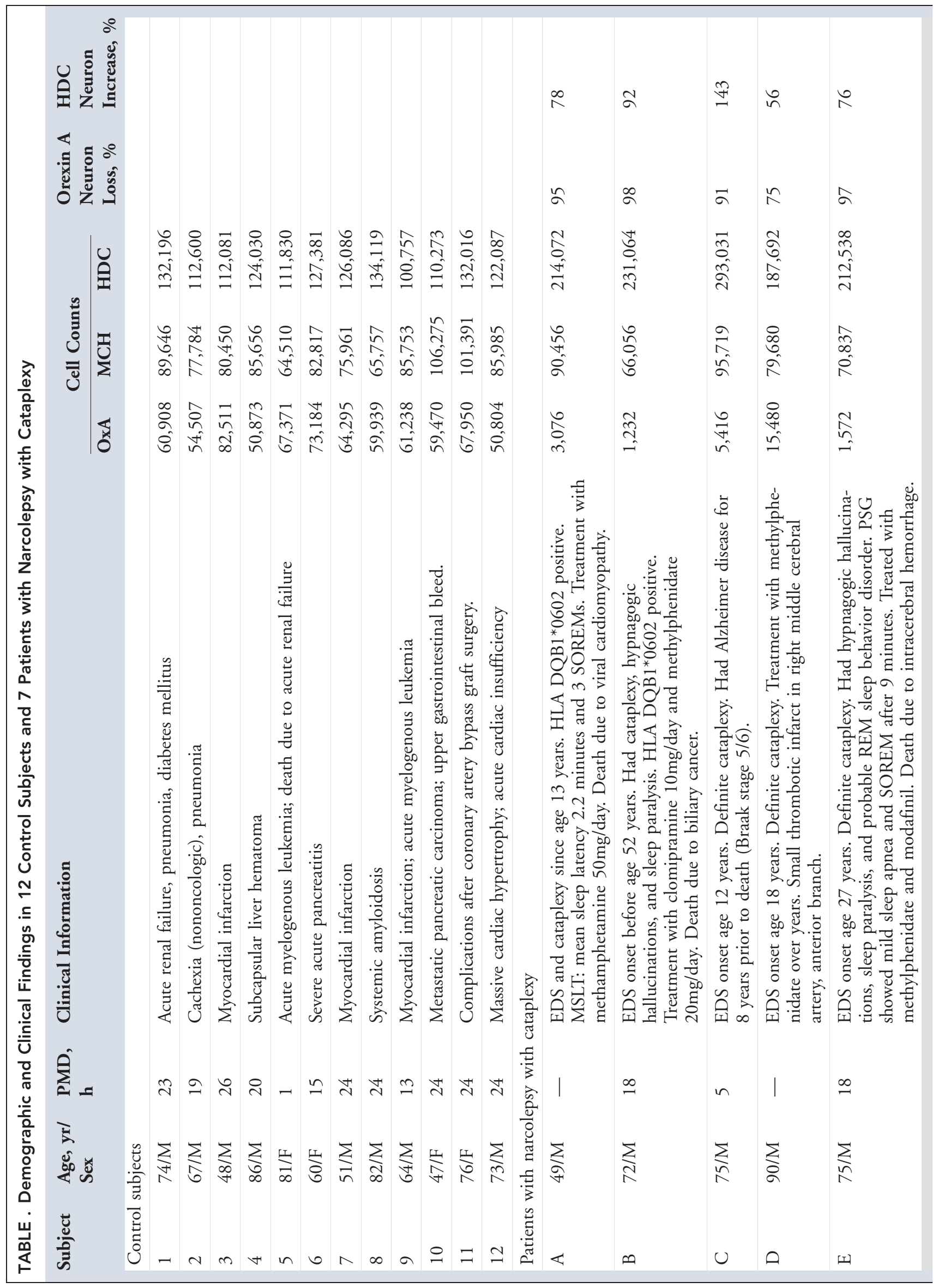




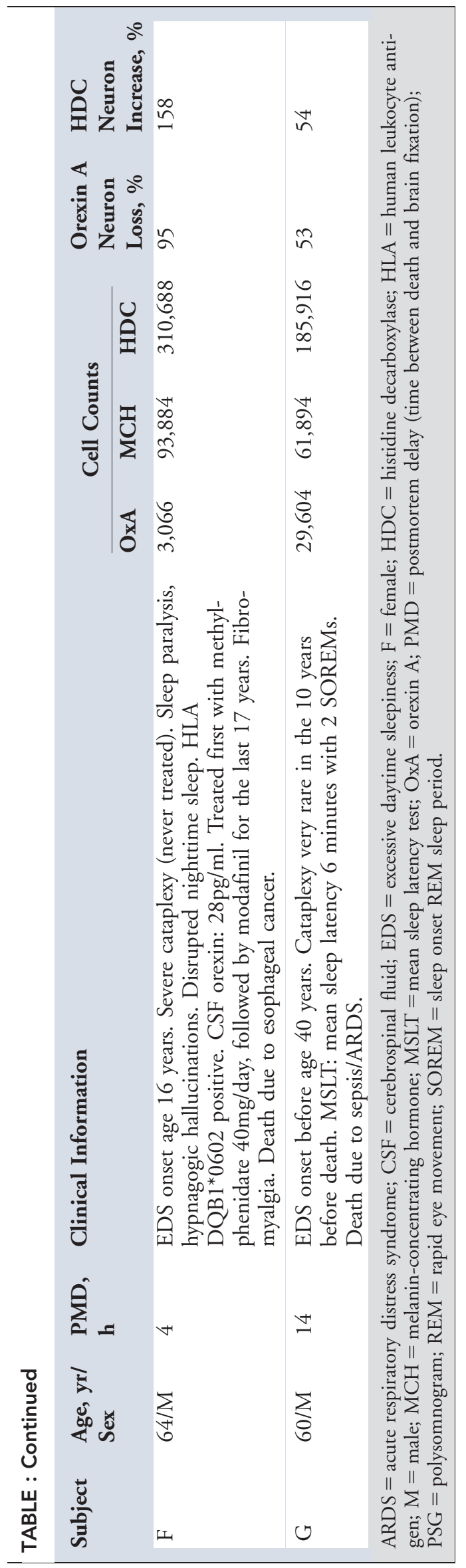

sucrose and $10 \%$ formalin. We then coronally sectioned the brains at $30 \mu \mathrm{m}$ on a freezing microtome into a 1:3 series.

\section{Immunohistochemistry on Human Tissue}

We immunostained hypothalamic sections for orexin A (hypocretin-1) as previously described. ${ }^{19}$ Briefly, free-floating sections were washed $3 \times$ with PBS and then reacted for 30 minutes with $0.3 \%$ hydrogen peroxide to block endogenous peroxidase activity. After several PBS washes and blocking for 1 hour in $3 \%$ normal horse serum, we incubated sections overnight in goat anti-orexin A antiserum (1:5,000; lot \# B0309; Santa Cruz Biotechnology, Santa Cruz, CA) and then for 1 hour in biotinylated donkey antigoat secondary antiserum (1:500; Jackson ImmunoResearch, West Grove, PA). After washing with PBS, we reacted sections for 1 hour with avidin/biotin complex (ABC Kit; Vector Laboratories, Burlingame, CA), and finally exposed them to nickel-enriched 3,3'-diaminobenzidine and $0.1 \%$ hydrogen peroxide to produce dark blue-black labeling of orexin neurons.

We used antigen retrieval to improve immunostaining for $\mathrm{MCH}$ and HDC, a marker of the histaminergic neurons of the tuberomammillary nucleus. After the initial reaction with hydrogen peroxide, we put sections in Tris-buffered saline (TBS; pH 7.6 for $\mathrm{MCH}, \mathrm{pH} 11.0$ for HDC immunostaining) at $95^{\circ} \mathrm{C}$ for 20 minutes. After cooling to room temperature, we washed the sections twice in TBS for 10 minutes, and then continued with the blocking step.

For $\mathrm{MCH}$ immunostaining, we used rabbit anti-MCH primary antiserum (1:2,500; a kind gift from Dr. E. MaratosFlier $^{27}$ ) followed by incubation with biotinylated donkey antirabbit secondary antiserum (1:500; Jackson ImmunoResearch). For HDC immunostaining, we used rabbit anti-HDC primary antiserum (1:800; raised against amino acids $1-481$ of the rat HDC peptide, catalog \# 03-16045, lot \# 112078; American Research Products, Waltham, MA), followed by incubation with biotinylated donkey antirabbit secondary antiserum (1:500; Jackson ImmunoResearch). The remaining steps for $\mathrm{MCH}$ and HDC immunostaining were the same as for orexin A immunostaining. We used the same lot of each primary antiserum for staining all control and narcoleptic brains.

\section{Immunohistochemistry on Mouse Tissue}

We used very similar immunohistochemical techniques for staining mouse sections. Dilutions of primary antisera were 1:5,000 for orexin A, 1:10,000 for $\mathrm{MCH}$, and 1:5,000 for HDC. Antigen retrieval was not necessary, because fixation time was short.

\section{Immunohistochemistry Controls}

The orexin and $\mathrm{MCH}$ antisera produced no staining in the brains of orexin $\mathrm{KO}$ and $\mathrm{MCH} \mathrm{KO}$ mice, respectively. The HDC antiserum produced no staining in the TMN of HDC $\mathrm{KO}$ mice, but it labeled monoamine neurons in the substantia nigra, ventral tegmental area, and dorsal raphe, perhaps by cross-reacting with aromatic L-amino acid decarboxylase. ${ }^{28}$ As these other monoamine nuclei are outside the TMN region, 
they did not affect TMN cell counts. In human hypothalami and brainstem, the HDC antiserum did not produce any ectopic labeling except for light labeling of occasional, small neurons in the periventricular nucleus of the rostral hypothalamus of 3 control subjects and 1 narcolepsy patient. These neurons do not produce HDC mRNA and they are well outside the TMN region, ${ }^{29,30}$ so they were not included in cell counts.

\section{Stereological Cell Counts}

Estimation of the total number of orexin A, MCH, and HDC labeled neurons was performed using a Zeiss (Oberkochen, Germany) Axiophot microscope with a motorized stage interfaced with a Stereo Investigator system (MBF Biosciences, Willston, VT). The same researcher (P.O.V.) did all counts to avoid interindividual variations. The HDC cell counts were repeated in a blinded fashion by Y.V.G. with very similar results; P.O.V. found $94 \%$ more HDC neurons in the narcolepsy subjects compared to the controls, compared to $86 \%$ more when counted by Y.V.G. Intraclass correlation coefficient was 0.948 , indicating high inter-rater reliability $(95 \%$ confidence interval $=0.860-0.981, p<0.001)$. In each section, a low-power objective lens $(\times 2.5)$ was used to manually delineate the boundaries around all positively labeled neurons. Actual counting was done using an oil immersed $\times 100$ objective. We counted neurons according to Gundersen's unbiased counting rules, with an optical dissector height of $10 \mu \mathrm{m} .{ }^{31,32}$ To achieve accurate cell counts as determined by a Gundersen's coefficient of error $<10 \%$, we chose a small counting grid of $150 \times 150 \mu \mathrm{m}$ and $100 \times 100 \mu \mathrm{m}$ in human and mouse tissue, respectively, and a counting frame of $50 \times 50 \mu \mathrm{m}$. Finally, the optical fractionator method provided the estimates of the total cell number, which was based on the abovementioned sampling parameters, the measured section thickness, and the sample frequency (1:24 series for human; 1:3 for mouse). For the human tissue, the coefficients of error were $8 \%$ (orexin A), $6 \%$ $(\mathrm{MCH})$, and 5\% (HDC). For the mouse tissue, the coefficients of error were $7 \%$ (orexin $\mathrm{A}$ ), $6 \%(\mathrm{MCH})$, and 6\% (HDC).

In the 12 control brains, the right and left halves of the hypothalamus contained similar numbers of orexin A neurons $(31,126 \pm 6,240$ vs $31,628 \pm 4,084), \quad \mathrm{MCH}$ neurons $(41,698 \pm 6,554$ vs $41,801 \pm 6,174)$, and histaminergic neurons $(60,513 \pm 6,226$ vs 59,942 $\pm 6,173$; Supplementary Fig 1$)$. Therefore, to compare total neuron number between NC patients and controls, we duplicated the cell count in those NC patients in whom only 1 side of the hypothalamus was available. The mean number of sections containing HDC-immunoreactive (IR) neurons was similar in control and narcolepsy subjects (11.2 \pm 2.4 vs $11.0 \pm 2.2, p=0.86)$.

We used the nucleator probe with 6 rays for calculation of the mean cell area. ${ }^{33}$ We placed the origin of the rays in the cell center and marked each intersecting point on the cell membrane. Histogram analysis revealed a normal distribution of cell sizes.

\section{Statistics}

Group data are reported as means and standard deviations. We compared cell counts between groups using Student $t$ test or analy- sis of variance (ANOVA) and performed correlation analyses using the Pearson test. Statistical significance was accepted at $p<0.05$.

\section{Results}

\section{Cell Counts in NC and Control Hypothalami}

NC patients had a substantial loss of orexin neurons. Specifically, the total number of orexin A-IR neurons in the NC patients was $8,492 \pm 10,515$, whereas it was $62,754 \pm 9,176$ in the control subjects $(p<0.001$; Fig 1). In $5 \mathrm{NC}$ patients, we found the characteristic, very severe orexin cell loss of 91 to $98 \%$, but 2 NC patients had partial orexin cell loss of only $75 \%$ and $53 \%$. The severity of orexin cell loss was not correlated with age at death or duration of $\mathrm{NC}$.

Surprisingly, NC patients had almost twice as many HDC-IR neurons as controls $(233,572 \pm 49,476$ vs $120,455 \pm 10,665$, respectively, $p<0.001)$. Every NC patient showed this increase, and there was no overlap between the number of HDC-IR neurons in NC patients and controls. Interestingly, the number of HDC-IR neurons was highest in the $5 \mathrm{NC}$ patients with $>90 \%$ orexin neuron loss compared to the $2 \mathrm{NC}$ patients with less severe orexin cell loss $(252,279 \pm 46,264$ vs $186,804 \pm 1,256, p=0.03)$. This increase of HDC-IR neurons was present across the entire tuberomammillary nucleus with no bias toward the anterior or posterior regions (Fig 2). The distribution and appearance of HDC-IR neurons in the narcolepsy subjects was very similar to that of controls and prior reports (Figs 3 and 4). ${ }^{29,30}$ The mean cell area of HDC-IR neurons was slightly (7\%) larger in $\mathrm{NC}$ patients than in controls $\left(766 \pm 179 \mu \mathrm{m}^{2}\right.$ vs $\left.715 \pm 180 \mu \mathrm{m}^{2}, p<0.001\right)$.

As expected from prior research, ${ }^{1,2}$ the number of MCH-IR neurons did not differ between controls and NC patients $(83,499 \pm 12,311$ vs 79,789 $\pm 13,876, p=0.57)$.

The quality of immunostaining for HDC, orexin $\mathrm{A}$, and $\mathrm{MCH}$ was excellent, with well-defined soma, proximal dendrites, and axons (see Fig 4 and Supplementary Figs 2 and 3).

\section{Cell Counts in Normal Mice and Mouse Models of Narcolepsy}

WT mice had 5,264 \pm 456 orexin A neurons. As expected, orexin $\mathrm{KO}$ mice had no orexin A-IR neurons (Fig 5). Atx mice had only $768 \pm 157$ orexin A-IR neurons, reflecting an 80 to $88 \%$ reduction $(p<0.001)$; these remaining cells were often small, with much less dendritic and axonal immunolabeling. Specifically, the mean cell area of orexin A-IR neurons in Atx mice was smaller by $34 \%$ than in WT mice $\left(133 \pm 37 \mu \mathrm{m}^{2}\right.$ vs $203 \pm 56 \mu \mathrm{m}^{2}$, respectively, $\left.p<0.001\right)$.

Orexin $\mathrm{KO}$ mice had $53 \%$ more HDC-IR neurons than WT mice $(5,352 \pm 977$ vs 3,508 \pm 703$)$. Atx mice showed an intermediate $28 \%$ increase $(4,478 \pm 1,143)$. The 

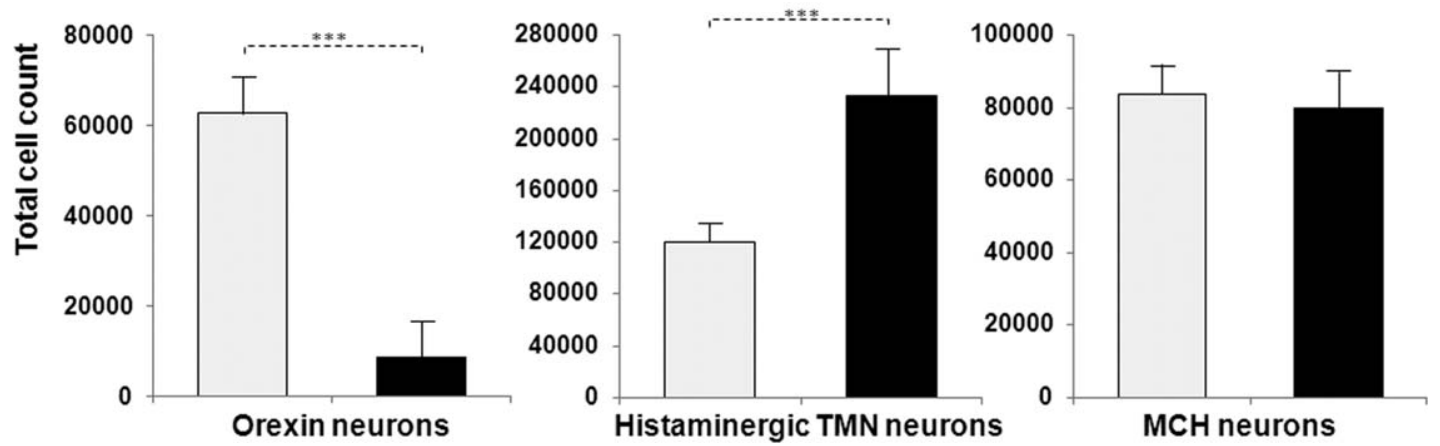

FIGURE 1: Compared to control subjects, patients with narcolepsy with cataplexy have $86 \%$ fewer orexin neurons and $94 \%$ more histaminergic tuberomammillary nucleus (TMN) neurons. The number of neurons producing melanin-concentrating hormone $(\mathrm{MCH})$ does not differ between the 2 groups. ${ }^{\star \star \star} p<0.001$.

number of HDC-IR neurons differed significantly across the 3 groups ( $p=0.02$ by ANOVA). Orexin KO mice had significantly more HDC-IR neurons than WT mice (post hoc adjustment with Bonferroni correction, $p=0.02$ ), but the increase in Atx mice did not reach statistical significance perhaps because of variability in orexin neuron loss. Atx mice with the greatest loss of orexin A-IR neurons tended to have more HDC-IR neurons, but the correlation between the numbers of orexin A-IR and HDC-IR neurons was not statistically significant (rho $=-0.40, p=0.43$ ). We did not find any significant association between orexin A-IR cell counts and the age at which the Atx mice were euthanized $(r=-0.51, p=0.30)$. The mean cell area of HDC-IR neurons was similar in the 3 mouse groups.

The numbers of $\mathrm{MCH}$ neurons were similar in WT, orexin KO, and Atx mice (5,185 \pm 612 , $6,063 \pm 1,052$, and 6,084 \pm 651 , respectively, $p=0.13$ )

The quality of immunostaining for orexin A, HDC, and MCH in mouse brains was excellent, with well-defined soma, proximal dendrites, and axons (Supplementary Figs 4-6).

\section{Discussion}

We found that patients with narcolepsy have almost twice as many histaminergic neurons as controls, and this increase was greatest in narcolepsy patients with severe loss of the orexin neurons $(>90 \%)$ compared to those with less severe loss. Similarly, mice completely lacking the orexin peptides had an increase in histaminergic neurons, and mice with a partial loss of the orexin neurons had a small increase.

This increase in histaminergic neurons is surprising, as loss of the orexin neurons was thought to be an adequate explanation for the symptoms of narcolepsy. This assumption was built on the hypothesis that the neuropathologic process that kills the orexin neurons is highly selective, with no involvement of adjacent cells such as those producing $\mathrm{MCH} .{ }^{1,2}$ In addition, loss of orexin signaling in mice, rats, and dogs seemed sufficient to produce the major symptoms of narcolepsy, including sleepiness and cataplexy. ${ }^{34,35}$ Although simple loss of the orexin neurons provides an explanation for many aspects of narcolepsy, our new findings indicate that the neuropathology of narcolepsy is more complicated, with unexpected involvement of another key arousal system.

\section{Technical Considerations}

The increase in histaminergic neurons appears robust, as it occurs in both people and mice with narcolepsy, but some methodological limitations should be kept in mind. First, our antiserum targeting a fragment of the rat HDC protein might also bind to an antigen other than HDC. In human brains, we saw very little ectopic labeling. In mouse brains, the HDC antisera labeled other monoamine neurons, but these were outside the hypothalamus and did not confound cell counts. In addition, the anatomical distribution and morphology of histaminergic neurons in narcoleptic subjects and mice are identical to those previously reported in healthy humans and rodents. ${ }^{29,30,36-38}$ Furthermore, John and colleagues have

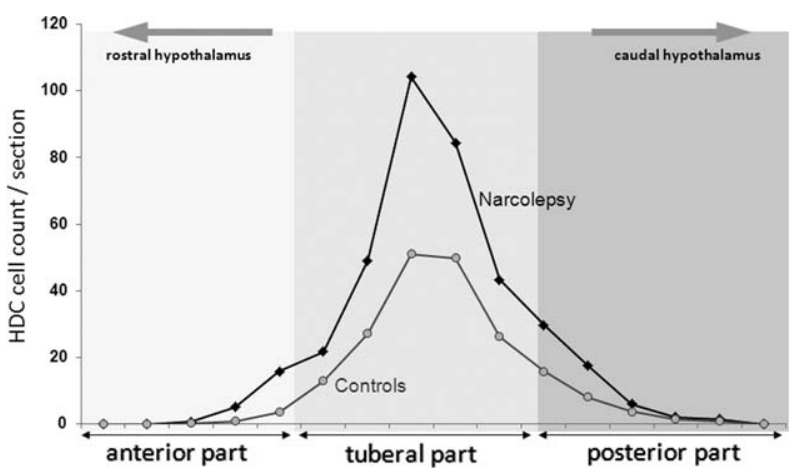

FIGURE 2: Rostrocaudal distributions of histaminergic tuberomammillary nucleus (TMN) neurons in control subjects and in narcolepsy patients. The distribution of histaminergic TMN neurons in narcolepsy patients follows the same pattern as in controls. HDC = histidine decarboxylase. 


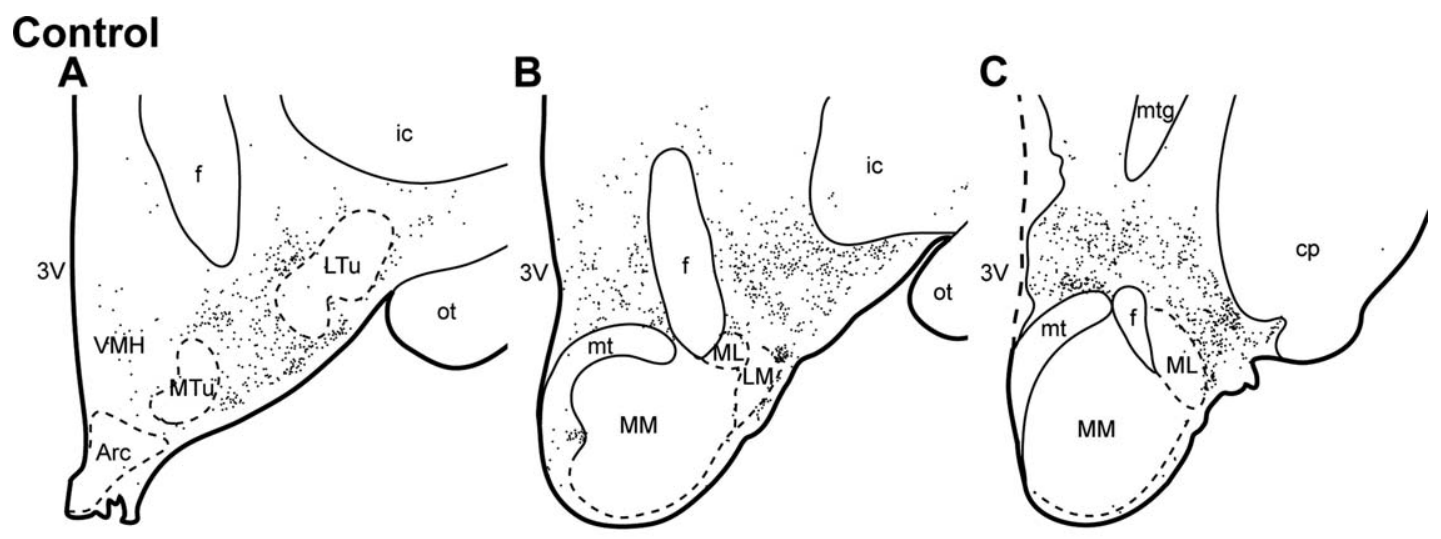

Narcolepsy

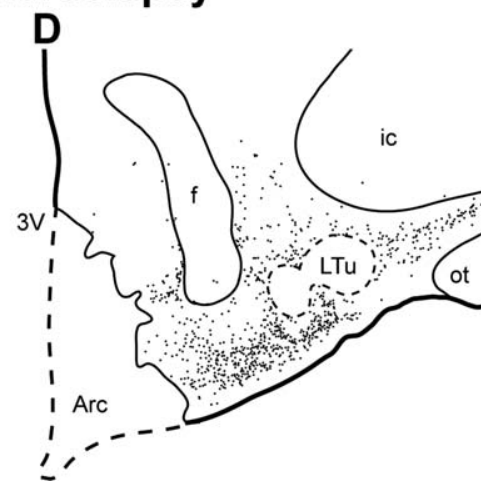

E

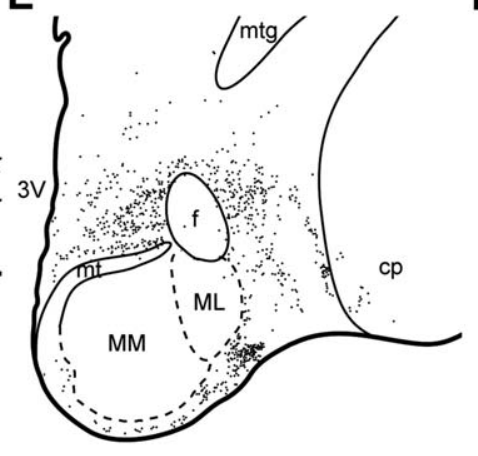

F

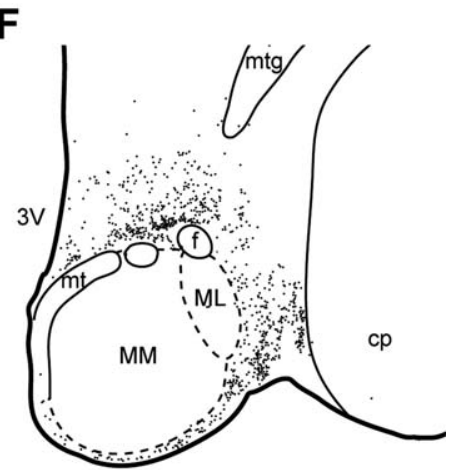

FIGURE 3: Compared to the tuberomammillary nucleus (TMN) of a control subject (A-C), the TMN of a patient with narcolepsy (D-F) has substantially more neurons expressing histidine decarboxylase (HDC). Each dot represents 1 HDC-immunoreactive neuron. In these drawings, the narcolepsy subject has $51 \%$ more HDC neurons than the control subject. $3 \mathrm{~V}=$ third ventricle; Arc $=$ arcuate nucleus; $c p=$ cerebral peduncle; $f=$ fornix; ic $=$ internal capsule; $L M=$ lateral mammillary nucleus; $L T u=$ lateral tuberal nucleus; $\mathrm{ML}=$ medial mammillary nucleus, lateral part; $\mathrm{MM}=$ medial mammillary nucleus, medial part; $\mathrm{mt}=\mathrm{mammillo-}$ thalamic tract; $\mathrm{mtg}=$ mammillotegmental tract; $\mathrm{MTu}=$ medial tuberal nucleus; ot =optic tract; $\mathrm{VMH}=$ ventromedial nucleus of the hypothalamus.

independently found a similar increase in histaminergic neurons in narcolepsy using antiserum targeting a short fragment of the human HDC protein. ${ }^{39}$ Nonetheless, future experiments should examine labeling for histamine, HDC mRNA, and other markers to determine additional neurochemical characteristics of these cells.

The postmortem delay before brain fixation tended to be shorter in patients with narcolepsy, and this could enhance HDC immunoreactivity. We think this is an unlikely cause of the large differences in number of HDC-IR neurons between groups, as there was no correlation between postmortem interval and HDC cell number in controls. In addition, some control brains had postmortem intervals similar to those of the narcolepsy brains, yet the number of HDC neurons was much lower than seen in any of the narcolepsy brains.

\section{Potential Mechanisms Underlying the Increase in Histaminergic Neurons}

Several mechanisms could underlie the increase in histaminergic neurons. In narcolepsy, the histaminergic neu- rons are distributed around the mammillary region in a normal pattern, so it seems most likely that HDC expression increases in narcolepsy, revealing some histaminergic neurons that may be difficult to detect in control subjects. Chronic loss of excitatory input from the orexin neurons may cause an upregulation of HDC similar to the increased expression of choline acetyltransferase reported in the laterodorsal tegmental nucleus of mice lacking orexin receptors. ${ }^{40}$ The TMN is clearly capable of plastic changes, as histamine levels and histaminergic fiber density increase during hibernation in ground squirrels. ${ }^{41}$ Alternatively, the increase in HDC neurons could be due to a change in neurochemical phenotype, as occurs in histaminergic and other monoaminergic neurons during development. ${ }^{42-44}$ The HDC gene is active in parts of the thalamus and other neurons outside the TMN early in development, ${ }^{45,46}$ and it will be important to examine these regions in future studies, as this ectopic expression could affect clinical symptoms. This neurotransmitter switching can occur even in adult animals and may serve a function similar to homeostatic synaptic 

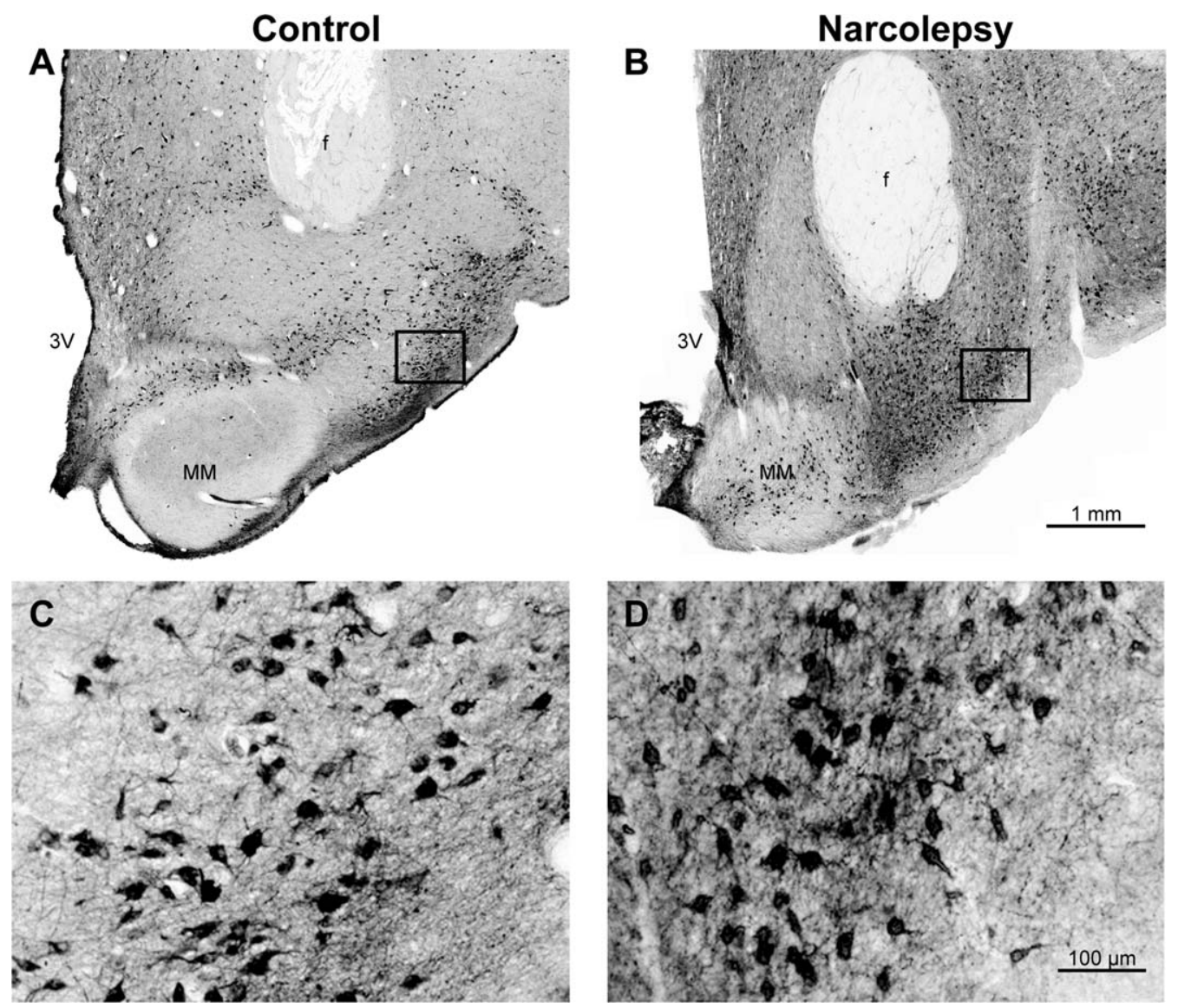

FIGURE 4: Histidine decarboxylase (HDC) immunostaining in control and narcolepsy subjects. (A, B) Sections near the rostral edge of the medial mammillary nucleus show approximately $60 \%$ more HDC-immunoreactive neurons in a subject with narcolepsy. In the section from a narcolepsy subject, some of the HDC neurons are scattered just anterior to the most rostral edge of the mammillary body but are not present within the mammillary body in more caudal sections. (C, D) HDC labeling is robust in neuronal soma, proximal dendrites, and axons. $3 \mathrm{~V}=$ third ventricle; $\mathrm{MM}=$ medial mammillary nucleus, medial part.

scaling. ${ }^{47}$ A third possibility is that chronic treatment with stimulants could alter TMN neurons, ${ }^{48,49}$ but this seems unlikely, as the untreated mice lacking orexin show similar increases in HDC neurons.
An important remaining question is whether the increase in HDC neurons occurs before or after the loss of orexin neurons. Most likely, the increase occurs after the loss of the orexin neurons, perhaps as a response to

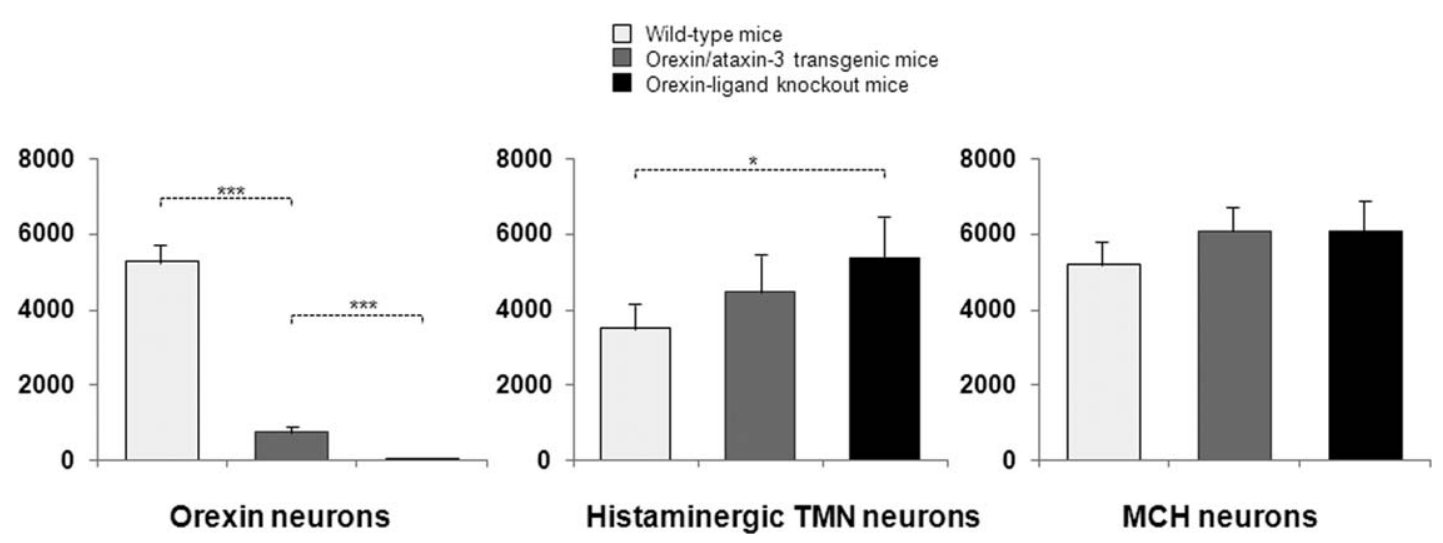

FIGURE 5: Numbers of orexin, histaminergic, and melanin-concentrating hormone (MCH) neurons in wild-type mice and 2 mouse models of narcolepsy. Orexin-ligand knockout mice have no orexin immunoreactivity and $53 \%$ more histaminergic neurons than wild-type mice. Orexin/ataxin-3 transgenic mice have an $85 \%$ loss of orexin neurons and $28 \%$ more histaminergic neurons than wild-type mice. The number of $\mathrm{MCH}$ neurons is similar in all groups. ${ }^{\star} p<0.05,{ }^{\star \star \star} p<0.001$. TMN $=$ tuberomammillary nucleus. 
reduced excitatory inputs. However, if the increase in histaminergic neurons occurs earlier, it might play some role in the destruction of the orexin neurons. In developing zebrafish, reduced histamine signaling decreases the number of orexin neurons. ${ }^{50}$ Although the direction of this response is different than in our study, it highlights the important interactions between these systems, and future studies examining the temporal relation between orexin neuron loss and HDC neuron increase would be very helpful to better understand the sequence of events.

Finally, one may speculate that orexin deficiency in narcolepsy could improve survival of histaminergic TMN neurons. Neurofibrillary tangles and plaques can occur in the TMN region in Alzheimer disease, ${ }^{51-53}$ and an orexin antagonist can reduce extracellular levels of amyloid beta. ${ }^{54}$ However, none of our control subjects had clinical or neuropathological evidence of Alzheimer disease. Furthermore, HDC mRNA does not vary with age, ${ }^{55}$ and the numbers of histaminergic neurons in our subjects did not correlate with age. Considering these findings and the nearly 2 -fold difference in the number of histaminergic neurons between control and narcolepsy subjects, we think it is much more likely that histaminergic neurons are increased in narcolepsy.

\section{Potential Impact of the Increase in Histaminergic Neurons}

The increase in histaminergic neurons may help compensate for reductions in orexin signaling. Hypersomnia (sleeping for excessively long periods) is common around the onset of narcolepsy, but over months to years, most patients sleep normal amounts. ${ }^{56}$ Similarly, acute pharmacologic antagonism of orexin signaling increases sleep in people and rodents. ${ }^{57}$ Perhaps acute reductions in orexin signaling reduce histamine signaling and cause hypersomnia, but chronic orexin deficiency in narcolepsy triggers increased expression of HDC that helps restore histamine signaling and normal amounts of wakefulness. How this actually affects synaptic concentrations of histamine remains unclear, as some studies report low CSF levels of histamine in narcolepsy, ${ }^{14-16}$ whereas others report that histamine levels are normal. ${ }^{58,59}$ In future research, it will be very useful to examine whether CSF histamine levels are low close to the onset of narcolepsy but increase at later times. Drugs to enhance histamine signaling (eg, tiprolisant) are now under development as a new class of wake-promoting medications, ${ }^{60}$ and if the number of histaminergic neurons increases slowly, then these drugs might be most effective in patients with longstanding narcolepsy.

The increase in histaminergic neurons may also shed light on some clinical aspects of narcolepsy. For example, TMN neurons remain active during cataplexy, ${ }^{61}$ and altered histaminergic signaling could explain the paradoxical preservation of consciousness during rapid eye movement (REM) sleep-like states such as cataplexy and sleep paralysis. In support of this perspective, Atx mice have a partial loss of orexin neurons and a smaller increase in histaminergic neurons than orexin $\mathrm{KO}$ mice, and behaviorally, they have less cataplexy and more REM sleep during the active period. ${ }^{24}$ Perhaps treatment of a narcolepsy patient with a histamine antagonist would reduce cataplexy and other intermediate states and increase transitions into full REM sleep. In addition, although all patients with narcolepsy have daytime sleepiness, many also have fragmented sleep at night. This could be caused by excessive histaminergic signaling during the sleep period that could lower the threshold to transition from sleep to wake. ${ }^{22}$

\section{Conclusions and Future Directions}

We found that the number of histaminergic TMN neurons is substantially increased in subjects with narcolepsy when compared with controls, especially in those with the most severe loss of the orexin neurons. Orexin KO mice also showed an increase in histaminergic neurons, and increased histaminergic signaling may influence the symptoms and clinical management of narcolepsy.

These observations raise many new questions and opportunities. For example, what mechanism triggers the increase in histaminergic neurons? When does the increase occur? Is the effect limited to only the histaminergic neurons or are there changes in other arousal systems? Research shedding light on these questions could easily lead to novel and more effective therapies for treating narcolepsy.

\section{Acknowledgment}

These experiments were supported by a research grant from the Swiss National Science Foundation(32003B_125504) to C.R.B., and a research grant from Wake Up Narcolepsy to T.E.S.

We thank Drs G. Rosen and L. Slomianka for use of their Stereo Investigator software, and Dr E. MaratosFlier for the $\mathrm{MCH}$ antiserum. The orexin $\mathrm{KO}$ and Atx mice were kind gifts from Drs T. Sakurai and M. Yanagisawa. Brains from HDC KO mice were a generous gift from Drs K. Yanai and T. Yoshikawa.

\section{Potential Conflicts of Interest}

E.M.: board membership, Jazz Pharmaceuticals; consultancy, Jazz Pharmaceuticals, Novo Nordisk; speaking fees, Vox Media. T.E.S.: consultancy, Merck, Ferrer Pharma, 
Concert Pharma, Purdue Pharma, Cereve; expert testimony, Ryan Ryan Deluca; speaking fees, Vox Media; royalties, Up to Date; paid educational presentations, Division of Sleep Medicine.

\section{References}

1. Peyron C, Faraco J, Rogers W, et al. A mutation in a case of early onset narcolepsy and a generalized absence of hypocretin peptides in human narcoleptic brains. Nat Med 2000;6:991-997.

2. Thannickal TC, Moore RY, Nienhuis R, et al. Reduced number of hypocretin neurons in human narcolepsy. Neuron 2000;27:469474.

3. Nishino S, Ripley B, Overeem S, et al. Hypocretin (orexin) deficiency in human narcolepsy. Lancet 2000;355:39-40.

4. Haas HL, Sergeeva OA, Selbach O. Histamine in the nervous system. Physiol Rev 2008;88:1183-1241.

5. Peyron C, Tighe DK, van den Pol AN, et al. Neurons containing hypocretin (orexin) project to multiple neuronal systems. J Neurosci 1998;18:9996-10015.

6. Eriksson KS, Sergeeva O, Brown RE, Haas HL. Orexin/hypocretin excites the histaminergic neurons of the tuberomammillary nucleus. J Neurosci 2001;21:9273-9279.

7. Torrealba F, Yanagisawa M, Saper CB. Colocalization of orexin A and glutamate immunoreactivity in axon terminals in the tuberomammillary nucleus in rats. Neuroscience 2003;119:1033-1044.

8. Mochizuki $T$, Arrigoni E, Marcus JN, et al. Orexin receptor 2 expression in the posterior hypothalamus rescues sleepiness in narcoleptic mice. Proc Natl Acad Sci U S A 2011;108:4471-4476.

9. Ko EM, Estabrooke IV, McCarthy M, Scammell TE. Wake-related activity of tuberomammillary neurons in rats. Brain Res 2003;992: 220-226.

10. Takahashi K, Lin JS, Sakai K. Neuronal activity of histaminergic tuberomammillary neurons during wake-sleep states in the mouse. J Neurosci 2006;26:10292-10298.

11. Zeitzer JM, Kodama T, Buckmaster CL, et al. Time-course of cerebrospinal fluid histamine in the wake-consolidated squirrel monkey. J Sleep Res 2012;21:189-194.

12. Roehrs TA, Tietz El, Zorick FJ, Roth T. Daytime sleepiness and antihistamines. Sleep 1984;7:137-141.

13. Lin JS. Brain structures and mechanisms involved in the control of cortical activation and wakefulness, with emphasis on the posterior hypothalamus and histaminergic neurons. Sleep Med Rev 2000;4:471-503.

14. Huang ZL, Qu WM, Li WD, et al. Arousal effect of orexin A depends on activation of the histaminergic system. Proc Natl Acad Sci U S A 2001:98:9965-9970.

15. Nishino S, Sakurai E, Nevsimalova S, et al. Decreased CSF histamine in narcolepsy with and without low CSF hypocretin-1 in comparison to healthy controls. Sleep 2009;32:175-180.

16. Kanbayashi $\mathrm{T}$, Kodama $\mathrm{T}$, Kondo $\mathrm{H}$, et al. CSF histamine contents in narcolepsy, idiopathic hypersomnia and obstructive sleep apnea syndrome. Sleep 2009;32:181-187.

17. Bassetti $\mathrm{CL}$, Baumann $\mathrm{CR}$, Dauvilliers $\mathrm{Y}$, et al. Cerebrospinal fluid histamine levels are decreased in patients with narcolepsy and excessive daytime sleepiness of other origin. J Sleep Res 2010;19: 620-623.

18. Ohtsu H, Watanabe T. New functions of histamine found in histidine decarboxylase gene knockout mice. Biochem Biophys Res Commun 2003;305:443-447.
19. Crocker A, España RA, Papadopoulou M, et al. Concomitant loss of dynorphin, NARP, and orexin in narcolepsy. Neurology 2005; 65:1184-1188

20. Scammell TE, Matheson JK, Honda M, et al. Coexistence of narcolepsy and Alzheimer's disease. Neurobiol Aging 2012;33:13181319

21. Chemelli RM, Willie JT, Sinton CM, et al. Narcolepsy in orexin knockout mice: molecular genetics of sleep regulation. Cell 1999. 98:437-451.

22. Mochizuki T, Crocker A, McCormack S, et al. Behavioral state instability in orexin knock-out mice. J Neurosci 2004;24:62916300

23. Hara J, Beuckmann CT, Nambu T, et al. Genetic ablation of orexin neurons in mice results in narcolepsy, hypophagia, and obesity. Neuron 2001;30:345-354.

24. Kantor S, Mochizuki T, Janisiewicz AM, et al. Orexin neurons are necessary for the circadian control of REM sleep. Sleep 2009;32: $1127-1134$

25. Chou TC, Lee CE, Lu J, et al. Orexin (hypocretin) neurons contain dynorphin. J Neurosci 2001;21:RC168.

26. Blouin AM, Thannickal TC, Worley PF, et al. Narp immunostaining of human hypocretin (orexin) neurons: loss in narcolepsy. Neurology 2005;65:1189-1192.

27. Pissios $P$, Ozcan $U$, Kokkotou E, et al. Melanin concentrating hormone is a novel regulator of islet function and growth. Diabetes 2007:56:311-319.

28. Mizuguchi J, Yabumoto M, Imamura I, et al. Immuno-cross-reactivity of histidine and dopa decarboxylases. Biochem Biophys Res Commun 1990;173:1299-1303.

29. Krolewski DM, Medina A, Kerman IA, et al. Expression patterns of corticotrophin-releasing factor, arginine vasopressin, histidine decarboxylase, melanin-concentrating hormone, and orexin genes in the human hypothalamus. J Comp Neurol 2010;518:4591-4611.

30. Airaksinen MS, Paetau A, Paljärvi $L$, et al. Histamine neurons in human hypothalamus: anatomy in normal and Alzheimer diseased brains. Neuroscience 1991:44:465-481.

31. Gundersen HJ, Jensen EB. The efficiency of systematic sampling in stereology and its prediction. J Microsc 1987;147:229-263.

32. West MJ. Stereological methods for estimating the total number of neurons and synapses: issues of precision and bias. Trends Neurosci 1999;22:51-61.

33. Gundersen HJ. The nucleator. J Microsc 1988;151:3-21.

34. Burgess CR, Scammell TE. Narcolepsy: neural mechanisms of sleepiness and cataplexy. J Neurosci 2012;32:12305-12311.

35. Sakurai T. Orexin deficiency and narcolepsy. Curr Opin Neurobiol 2013;23:760-766.

36. Panula P, Yang HY, Costa E. Histamine-concentrating neurons in the rat hypothalamus. Proc Natl Acad Sci U S A 1984;81:2572-2576.

37. Watanabe T, Taguchi $Y$, Shiosaka $S$, et al. Distribution of the histaminergic neuron system in the central nervous system of rats; a fluorescent immunohistochemical analysis with histidine decarboxylase as a marker. Brain Res 1984;295:13-25.

38. Karlstedt K, Nissinen M, Michelsen KA, Panula P. Multiple sites of L-histidine decarboxylase expression in mouse suggest nove developmental functions for histamine. Dev Dyn 2001;221:81-91.

39. John J, Thannickal TC, McGregor R, et al. Greatly increased numbers of histamine cells in human narcolepsy with cataplexy. Ann Neurol (in press)

40. Kalogiannis M, Grupke SL, Potter PE, et al. Narcoleptic orexin receptor knockout mice express enhanced cholinergic properties in laterodorsal tegmental neurons. Eur J Neurosci 2010;32:130142. 
41. Sallmen $T$, Beckman $A L$, Stanton $T L$, et al. Major changes in the brain histamine system of the ground squirrel citellus lateralis during hibernation. J Neurosci 1999;19:1824-1835.

42. Asmus SE, Parsons S, Landis SC. Developmental changes in the transmitter properties of sympathetic neurons that innervate the periosteum. J Neurosci 2000;20:1495-1504.

43. Bezin L, Marcel D, Desgeorges S, et al. Singular subsets of locus coeruleus neurons may recover tyrosine hydroxylase phenotype transiently expressed during development. Brain Res Mol Brain Res 2000;76:275-281.

44. Molina-Hernández A, Díaz NF, Arias-Montaño JA. Histamine in brain development. J Neurochem 2012;122:872-882.

45. Zecharia AY, Yu X, Götz T, et al. GABAergic inhibition of histaminergic neurons regulates active waking but not the sleep-wake switch or propofol-induced loss of consciousness. J Neurosci 2012;32:13062-13075.

46. Walker AK, Park WM, Chuang JC, et al. Characterization of gastric and neuronal histaminergic populations using a transgenic mouse model. PLoS One 2013;8:e60276.

47. Dulcis D, Jamshidi P, Leutgeb S, Spitzer NC. Neurotransmitter switching in the adult brain regulates behavior. Science 2013;340 449-453.

48. Ishizuka T, Sakamoto Y, Sakurai T, Yamatodani A. Modafinil increases histamine release in the anterior hypothalamus of rats. Neurosci Lett 2003;339:143-146.

49. Horner WE, Johnson DE, Schmidt AW, Rollema H. Methylphenidate and atomoxetine increase histamine release in rat prefrontal cortex. Eur J Pharmacol 2007;558:96-97.

50. Sundvik $M$, Kudo $H$, Toivonen $P$, et al. The histaminergic system regulates wakefulness and orexin/hypocretin neuron development via histamine receptor H1 in zebrafish. FASEB J 2011;25:43384347
51. Ishii T. Distribution of Alzheimer's neurofibrillary changes in the brain stem and hypothalamus of senile dementia. Acta Neuropathol 1966;6:181-187.

52. Saper CB, German DC. Hypothalamic pathology in Alzheimer's disease. Neurosci Lett 1987;74:364-370.

53. Nakamura S, Takemura M, Ohnishi K, et al. Loss of large neurons and occurrence of neurofibrillary tangles in the tuberomammillary nucleus of patients with Alzheimer's disease. Neurosci Lett 1993, 151:196-199.

54. Kang JE, Lim MM, Bateman RJ, et al. Amyloid-beta dynamics are regulated by orexin and the sleep-wake cycle. Science 2009;326: 1005-1007.

55. Shan L, Swaab DF, Bao AM. Neuronal histaminergic system in aging and age-related neurodegenerative disorders. Exp Geronto 2013;48:603-607.

56. Dauvilliers Y, Arnulf I, Mignot E. Narcolepsy with cataplexy. Lancet 2007:369:499-511.

57. Brisbare-Roch C, Dingemanse J, Koberstein R, et al. Promotion of sleep by targeting the orexin system in rats, dogs and humans. Nat Med 2007:13:150-155.

58. Croyal M, Dauvilliers $Y$, Labeeuw $O$, et al. Histamine and telemethylhistamine quantification in cerebrospinal fluid from narcoleptic subjects by liquid chromatography tandem mass spectrometry with precolumn derivatization. Anal Biochem 2011;409:28-36.

59. Dauvilliers $Y$, Delallée N, Jaussent I, et al. Normal cerebrospina fluid histamine and tele-methylhistamine levels in hypersomnia conditions. Sleep 2012;35:1359-1366.

60. Lin JS, Dauvilliers $Y$, Arnulf I, et al. An inverse agonist of the histamine $\mathrm{H}$ (3) receptor improves wakefulness in narcolepsy: studies in orexin-/- mice and patients. Neurobiol Dis 2008;30:74-83.

61. John J, Wu MF, Boehmer LN, Siegel JM. Cataplexy-active neurons in the hypothalamus: implications for the role of histamine in sleep and waking behavior. Neuron 2004;42:619-634. 
Philipp 0. Valko

Ramin Khatami

Christian R. Baumann

Claudio L. Bassetti

\section{No persistent effect of intravenous immunoglobulins in patients with narcolepsy with cataplexy}

Received: 15 November 2007

Received in revised form: 12 April 2008

Accepted: 2 May 2008

Published online: 25 September 2008

P. O. Valko, MD · R. Khatami, MD .

C. R. Baumann, MD .

Prof. C. L. Bassetti, MD (可)

Dept. of Neurology

University Hospital of Zurich

Frauenklinikstrasse 26

8091 Zurich, Switzerland

Tel.: +41-44/255-5503

Fax: +41-44/255-4380

E-Mail: claudio.bassetti@usz.ch
Abstract We report on four patients with narcolepsy with cataplexy (NC), who were treated with high-dose intravenous immunoglobulins (IVIg). Although in some patients transient effects were seen of both objective (multiple sleep latency test and maintenance of wakefulness test) and subjective symptoms (Epworth Sleepiness Scale and frequency of cataplexy), these effects lasted at the most for a few weeks and did not persist. Our report challenges the recent observations of a favorable and persistent effect of IVIg in NC patients.

Key words narcolepsy · cataplexy · intravenous immunoglobulins · excessive daytime sleepiness

\section{Introduction}

The etiology of the selective loss of hypocretin (orexin) neurons in the posterolateral hypothalamus in sporadic narcolepsy with cataplexy (NC) is not known. Because of the tight association of narcolepsy with certain human leukocyte antigen (HLA) alleles, an autoimmune mechanism is considered $[1,2]$. Two groups recently reported on hypocretin-deficient NC patients, who experienced a significant and sustained reduction of frequency and severity of cataplexy after administration of high-dose intravenous immunoglobulins (IVIg), in particular when treated early after disease onset $[3,4,8]$. These findings seemed to corroborate the autoimmune hypothesis and suggested that early IVIg treatment in NC might favorably modify the course of disease.

In this report we describe four hypocretin-deficient $\mathrm{NC}$ patients without or with uncertain benefit from IVIg therapy.

\section{Case 1}

This 43-year-old woman noticed in summer 2004 the gradual appearance of daytime sleepiness with daily irresistible and restorative naps. In December 2004 she experienced the first cataplectic attacks involving the head and both lower limbs, triggered by strong emotions (mainly laughter), and occurring up to several times per day. Her night-time sleep was increasingly disturbed by frequent awakenings, and dreams became more vivid and realistic. She also had rare episodes of (hypnagogic) hallucinations and sleep paralysis. Epworth Sleepiness Scale (ESS) was 19/24, Ullanlinna Narcolepsy Score (UNS) 16/44, and Swiss Narcolepsy Scale (SNS) 36 [7, 10]. Clinical examination was unremarkable. Magnetic resonance imaging (MRI) scan of the brain showed few, nonspecific, small $(<1 \mathrm{~cm})$ hyperintense signal alterations of the subcortical/white matter without gadolinium enhancement. Cerebrospinal fluid (CSF) examination revealed normal glucose and protein content, 5 mononuclear cells $/ \mu \mathrm{l}$, and no oligoclonal bands. CSF hypocretin-1 was undetectable $(<40 \mathrm{pg} / \mathrm{ml})$. HLA typing was positive for $\mathrm{DQB} 1{ }^{*} 0602, \mathrm{DQA} 1^{*} 01$, DRB $1{ }^{\star} 15$ and DRB5*. Polysomnography (PSG), multiple 
sleep latency tests (MSLT; showing 4 sleep onset REM periods [SOREMP] in 5 tests) and maintenance of wakefulness tests (MWT; showing 2/4 SOREMPs) were consistent with narcolepsy (Fig. 1).

Methylprednisolone $1000 \mathrm{mg} / \mathrm{d}$ was administered intravenously for 3 consecutive days and then orally tapered over 8 days. During the first 3 weeks after steroid treatment the patient reported a mild (10-20\% better as estimated by the patient) but only transient reduction of EDS and cataplexy. This prompted a trial with IVIg at a dose of $1 \mathrm{~g} / \mathrm{kg} /$ day over 2 days, repeated three times at 5-week intervals (latency from cataplexy onset: 4 months). After the first two IVIg infusions, the patient reported a gradual improvement of EDS (decrease of ESS from 15-19/24 at baseline to 11/24 after the second IVIg trial) and a mild reduction of frequency of self-reported cataplexy (measured by a cataplexy diary). However, after the third IVIg infusion, cataplexy and severity
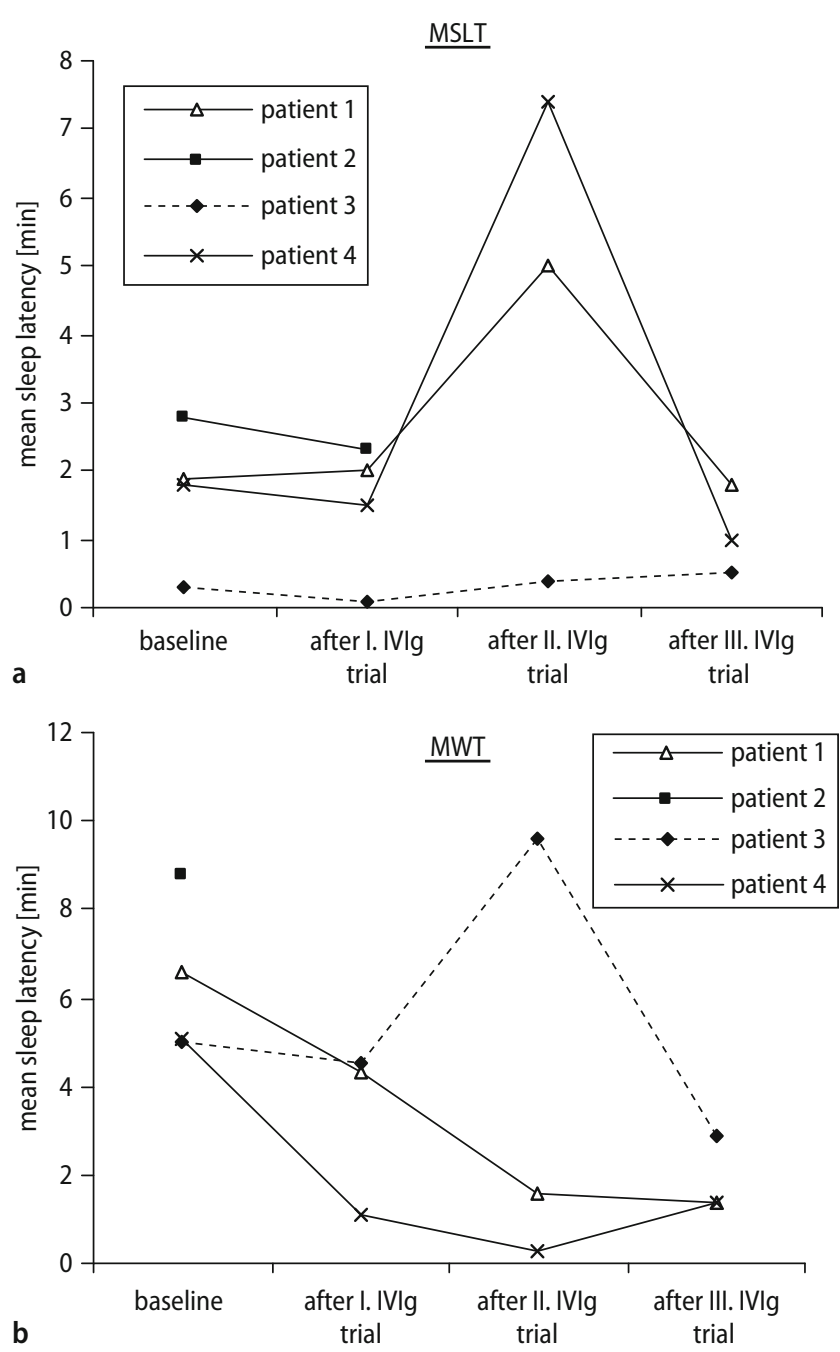

Fig. 1 a Multiple sleep latency test (MSLT) and $\mathbf{b}$ the maintenance of wakefulness test (MWT) at baseline and their response to IVIg treatment of EDS $(E S S=17 / 24)$ returned to pre-treatment levels. No effects were noted on hallucinations, sleep paralysis and MSLT/MWT results during and after IVIg (Fig.2).

\section{Case 2}

This 59-year-old woman developed EDS and cataplectic attacks after a flu-like infection in 1987. She had 3-4 short irresistible and restorative naps per day, her cataplectic attacks affected mainly the head and neck, triggered by laughter, but also by negative emotions, occurring up to 1-3 times/day. Acoustic (hypnagogic) hallucinations occurred once a month, and a few years later she also developed rare episodes of sleep paralysis. Most drugs, including modafinil, methylphenidate, dexamphetamine and mazindol, were not tolerated. Fluoxetine had a moderate effect on cataplexy.
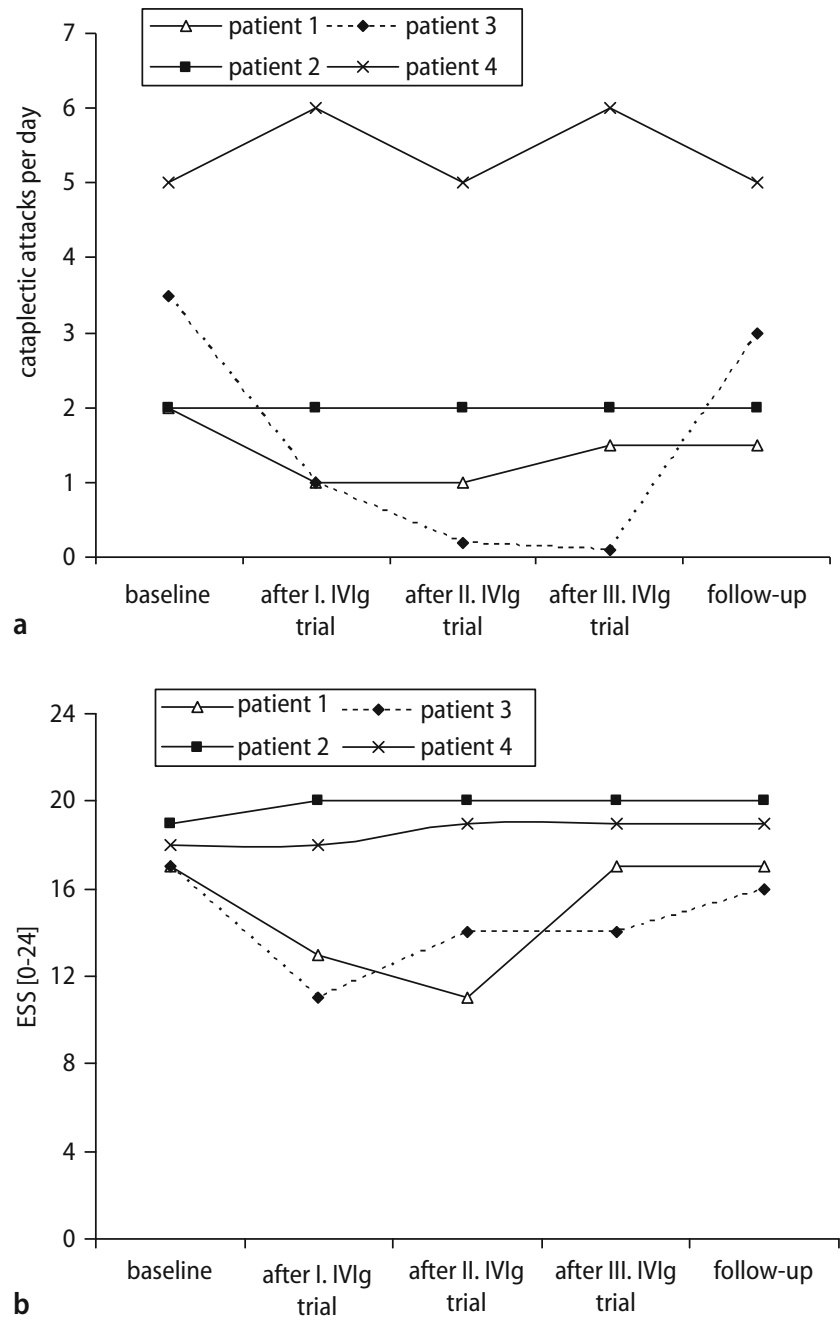

Fig. 2 a Frequency of self-reported cataplectic attacks and $\mathbf{b}$ Epworth Sleepiness Scale (ESS, measuring subjective sleepiness) at baseline and after each administration of intravenous immunoglobulins 
In August 2003 a common variable immunodeficiency syndrome (deficiency of IgG subclasses II and IV, [9]) was diagnosed and a treatment with IVIg infusions of $20 \mathrm{~g}$ every 3 weeks was started.

In September 2004 we first saw the patient. ESS was 20/24, UNS 30/44, and SNS -66. Clinical examination and brain MRI were unremarkable. Routine CSF examinations were normal. CSF hypocretin-1 was undetectable. HLA-typing was positive for $\mathrm{DQB} 1{ }^{*} 0602, \mathrm{DQA} 1{ }^{\star} 01$, DRB $1^{\star} 15$ and DRB5 ${ }^{\star}$. Sleep-wake tests (PSG, MSLT with 2/4 SOREMPs, MWT without SOREMPs) were typical for narcolepsy (Fig. 1).

Considering the failure of all previous medications and the diagnosis of a common-variable immunodeficiency syndrome, additional treatment with high-dose IVIg infusions was started (latency from narcolepsy onset: 17 years). Because of allergic reactions the usual dose $(2 \mathrm{~g} / \mathrm{kg}$ ) was distributed over 5 days (and not as usual over 2 days). No effects were noted on EDS, cataplexy, hallucinations, sleep paralysis, and MSLT/MWT results during and after IVIg (which was stopped after the second administration because of recrudescence of allergy) (Fig.1).

\section{Case 3}

This 41-year-old man developed EDS with irresistible naps within a few days in August 2004 while mastering an unusually stressful family situation. In the following months he experienced few generalized and several partial clear-cut cataplectic attacks per day which were triggered by laughing and other positive emotions, as well as occasional sleep paralysis and hypnagogic and hypnopompic hallucinations. Night-time sleep became more fragmentary and his dreams intense and threatening. ESS was 22/24, UNS 29/44, and SNS -95. Clinical examination and brain MRI were unremarkable. Sleepwake tests were typical for narcolepsy, including $4 / 4$ SOREMPs in MSLT and 3/4 SOREMPs in MWT (Fig.1). Repeated CSF examination showed a mild pleocytosis (4-10 mononuclear cells/ $\mu \mathrm{l}$ ), without oligoclonal bands. CSF hypocretin-1 was undetectable. HLA-typing was positive for $\mathrm{DQB} 1{ }^{\star} 0602, \mathrm{DQA} 1^{\star} 01, \mathrm{DRB} 1^{\star} 15$ and $\mathrm{DRB} 5 *$. Extensive serologic and immunologic tests were negative.

The combination of modafinil 100-200 mg b.i.d. with venlafaxine $75 \mathrm{mg}$ completely controlled cataplectic attacks, hallucinations and sleep paralysis and significantly improved EDS (the ESS went from 22 to 15). He was able to return to work in full time position for 2.5 years. In association with an increased psychosocial stress (divorce) and weight gain his narcoleptic symptoms exacerbated and became disabled despite several modifications of his medical therapy. This prompted treatment with IVIg infusions at a dose of $1 \mathrm{~g} / \mathrm{kg} /$ day over 2 days, repeated three times at 5-week intervals (latency disease onset: 4 years).

The patient reported a significant reduction in number and severity of cataplectic attacks from $3-4 / \mathrm{d}$ at baseline to $1-2 /$ week after the second and $0-1 /$ week after the third IVIg infusion. The overall improvement of cataplexy was estimated to be around $80-90 \%$. However, during the short hospitalizations on occasion of the IVIg trials, the persistent occurrence of daily cataplectic attacks could be witnessed by several of us (POV, RK, CLB). There was also a strong improvement of subjective EDS, which the patient reported to recognize already within the first hour of the first IVIg infusion (reduction of ESS from 15-19/24 at baseline to 11/24 after the first IVIg). Objective parameters (MSLT, MWT), however, remained unchanged. In the following weeks, the patient experienced also a progressive reappearance of narcoleptic symptoms. Eight weeks after the last IVIg trial severity of EDS, cataplexy and sleep paralysis and hallucinations were comparable to pre-treatment levels.

\section{Case 4}

In June 2006 this 52-year-old woman developed EDS with irresistible need of 2-3 restorative naps/day and severe cataplectic attacks with falls, triggered by both positive and negative emotions, and occurring up to ten times per day. She did not notice any change of her night-time sleep, but her dreams became more intensive. She has never experienced any hypnagogic hallucinations or sleep paralysis. ESS was $17 / 24$, UNS 39/44, and SNS -92. Clinical examination and brain MRI were unremarkable. Routine CSF examination was normal. CSF hypocretin-1 was undetectable. HLA-typing was positive for $\mathrm{DQB} 1{ }^{\star} 0602, \mathrm{DQA} 1^{\star} 01, \mathrm{DRB} 1^{*} 15$ and $\mathrm{DRB} 5^{\star}$. Sleep-wake tests were typical for narcolepsy, including 2/4 SOREMPs in MSLT tests and 3/4 SOREMPs in MWT (Fig.1).

Treatment with modafinil $50 \mathrm{mg} / \mathrm{d}$ and fluoxetine 20 $\mathrm{mg} / \mathrm{d}$ was started, leading only to a mild improvement of both subjective EDS and cataplexy. Treatment with modafinil and fluoxetine was gradually tapered, and after a drug-free period of 3 weeks a trial with IVIg was started at a dose of $1 \mathrm{~g} / \mathrm{kg} /$ day over 2 days, repeated three times at 5-week intervals (latency from cataplexy onset: 11 months). No effects were noted on EDS, cataplexy, and MSLT/MWT results during and after IVIg treatment.

\section{Discussion}

We report the absence of persistent improvements of narcoleptic symptoms in four patients treated with IVIg infusions. Although transient effects were seen concern- 
ing MSLT in patients 1 and 4, MWT in patient 3, cataplectic attacks in patient 3, and ESS in patients 1 and 3, these effects lasted at the most for a few weeks and did not persist. This observation is in contrast with the recent reports by Dauvilliers, Lecendreux, and collegues, on five narcolepsy patients $[3,4,8]$ in whom a sustained reduction in number and severity of cataplexy and a moderate effect on EDS (in all five patients) were achieved. The lack of a significant and persistent effect of intravenous steroids given early after onset of symptoms in one of our four patients also confirms the report of Mignot [6].

Four factors may account for the lack of significant effects of IVIg in our patients. First, only two of our patients were treated early after onset of cataplexy (four and eleven months, respectively, after the first appearance of cataplectic attacks). However, one patient described by Dauvilliers et al. was treated with IVIg with a delay of 9 years from disease onset, but reported improvement on both cataplexy and EDS with persistence of efficacy after 22 months without any anticataplectic or stimulant treatment. Second, in all of our patients CSF hypocretin-1 was already undetectable, suggesting that (presumed) autoimmune destruction of hypocretin neurons was already complete when IVIg therapy was started. However, all patients reported by Dauvilliers et al. had also undetectable CSF hypocretin-levels before IVIg treatment was started. Third, the treatment protocol was not identical in each patient, with patient 1 receiving corticosteroids and patient 2 receiving IVIg in lower dosages prior to the three IVIg trials. Fourth, our patients were older at disease onset $(39,40,41$ and 51 years) than those reported by Dauvilliers et al. $(10,12,21$ and 43 years). Both the efficacy of IVIg and the susceptibility to placebo effects may significantly depend on the patient's age at disease onset.

The discrepancy between lack of objective improvement (of EDS) and strong subjective improvement (of both cataplexy and EDS) in patient 1 and 3 suggest that a placebo effect might explain, at least in some cases, the improvement of narcoleptic symptoms (and particularly cataplexy) after IVIg. Indeed, a similar observation was recently reported in a narcoleptic patient who underwent a double-blind placebo-controlled administration of IVIg [5]. The authors could not find a difference between placebo and IVIg treatment. Furthermore, a recent trial with sodium oxybate has proven the existence of a placebo effect on cataplexy [11].

Our report challenges the first, very optimistic observations on the favorable effects of IVIg in patients with narcolepsy with cataplexy $[3,4,8]$, without proving the absence of any effect of IVIg (particularly on cataplexy). Only a randomized, double-blind and placebo-controlled study with narcolepsy patients with both early and late disease onset could determine the "true" value of IVIg in the treatment of narcolepsy and the ideal interval after onset of symptoms for this expensive and possibly harmful therapeutic intervention.

Conflict of interest The authors declare no conflict of interest.

\section{References}

1. Bassetti CL (2005) Narcolepsy: selective hypocretin (orexin) neuronal loss and multiple signaling deficiencies. Neurology 65:1152-1153

2. Crocker A, España RA, Papadopoulou M, Saper CB, Faraco J, Sakurai T, Honda M, Mignot E, Scammell TE (2005) Concomitant loss of dynorphin, NARP, and orexin in narcolepsy. Neurology 65:1184-1188

3. Dauvilliers Y, Carlander B, Rivier F, Touchon J, Tafti M (2004) Successful management of cataplexy with intravenous immunoglobulins at narcolepsy onset. Ann Neurol 56:905-908

4. Dauvilliers Y (2006) Follow-up of four narcolepsy patients treated with intravenous immunoglobulins. Ann Neurol $60: 153$
5. Fronczek R, Verschuuren J, Lammers GJ (2007) Response to intravenous immunoglobulins and placebo in a patient with narcolepsy with cataplexy. J Neurol 254:1607-1608

6. Hecht M, Lin L, Kushida CA, Umetsu DT, Taheri S, Einen M, Mignot E (2003) Report of a case of immunosuppression with prednisone in an 8-year-old boy with an acute onset of hypocretindeficiency narcolepsy. Sleep 26: 809-810

7. Hublin C, Partinen M, Kaprio J, Koskenvuo M, Guilleminault C (1994) Epidemiology of narcolepsy. Sleep 17: S7-S12
8. Lecendreux M, Maret S, Bassetti C, Mouren M-C, Tafti M (2003) Clinical efficacy of high-dose intravenous immunoglobulins near the onset of narcolepsy in a 10-year-old boy. J Sleep Res 12:347-348

9. Salzer U, Grimbacher B (2006) Common variable immunodeficiency: The power of co-stimulation. Semin Immunol 18:337-346

10. Sturzenegger C, Bassetti CL (2004) The clinical spectrum of narcolepsy with cataplexy: a reappraisal. J Sleep Res 13:395-406

11. The U.S. Xyrem Multicenter Study Group (2002) A randomized, double blind, placebo-controlled multicenter trial comparing the effects of three doses of orally administered sodium oxybate with placebo for the treatment of narcolepsy. Sleep 25:42-49 


\title{
Sodium Oxybate in Narcolepsy with Cataplexy: Zurich Sleep Center Experience
}

\author{
R. Poryazova ${ }^{\text {a }} \quad$ S. Tartarotti ${ }^{a}$ R. Khatami $^{\text {b }} \quad$ C.R. Baumann ${ }^{\text {a }} \quad$ P. Valko ${ }^{a}$ \\ U. Kallweit ${ }^{\mathrm{a}}$ E. Werth ${ }^{\mathrm{a}}$ C.L. Bassettia,c \\ ${ }^{a}$ Department of Neurology, University Hospital Zurich, Zurich, ${ }^{b}$ Sleep Clinic, Barmelweid, and \\ 'Department of Neurology, Neurocenter (EOC) of Southern Switzerland, Ospedale Civico, Lugano, Switzerland
}

\section{Key Words}

Narcolepsy $\cdot$ Cataplexy $\cdot$ Excessive daytime sleepiness $\cdot$

Sleep paralysis $\cdot$ Hallucinations $\cdot$ Sodium oxybate

\begin{abstract}
Sodium oxybate $\left(\mathrm{SO}\right.$; Xyrem $\left.{ }^{\circledR}\right)$ has been approved in most countries for treatment of narcolepsy and cataplexy. In this study, we present a single-center experience of a series of 18 patients with narcolepsy with cataplexy $(18 / 18 \mathrm{DQB} 1 * 0602$ positive, 17/17 with low/absent cerebrospinal fluid hypocretin) in whom SO was prescribed. After $26 \pm 13$ months, 13/18 patients were still on SO at a mean dosage of $6.1 \pm 1.2 \mathrm{~g}$ (in 8 of them in combination with stimulants). The following significant effects were observed: improved subjective sleepiness $(12 / 13)$, cataplexy (13/13; median number of attacks from 20 to $1 /$ month), hallucinations (8/10) and sleep paralysis (8/8); increase in mean sleep latency on the Maintenance of Wakefulness Test (from 5.5 to $17.4 \mathrm{~min}$ ) and sleep/rest efficiency on actigraphy (from 61 to 76\%); decrease in Epworth Sleepiness Scale score (from 18 to 14), sleep onset REM periods on the Multiple Sleep Latency Test (from 3.6 to 2.4) and errors in the Steer-Clear Test (from 11 to 2\%). Five patients discontinued SO because of insufficient compliance $(n=2)$,
\end{abstract}

lack of efficiency $(n=1)$ and side effects $(n=1)$. These data confirm and expand previous reports on the good effects and tolerability of SO as a treatment for narcolepsy with cataplexy.

Copyright $\odot 2011$ S. Karger AG, Basel

\section{Introduction}

Narcolepsy with cataplexy (NC) is a disabling life-long sleep-wake disorder, characterized by excessive daytime sleepiness (EDS) and sudden loss of muscle tone triggered by emotions (cataplexy) [1]. Additional symptoms include sleep paralysis, hypnagogic hallucinations and fragmented nighttime sleep. Cataplexy is specific for narcolepsy and its presence allows diagnosing NC on clinical grounds. Typical paraclinical findings with diagnostic significance include the occurrence of two or more sleeponset REM periods (SOREMPs) in the multiple sleep latency test (MSLT) [2], association to the HLA-DQB1*0602 (found in $98 \%$ of NC compared to $35 \%$ of healthy controls) [3] and decrease of CSF hypocretin (levels $<110 \mathrm{pg} /$ $\mathrm{ml}$ are considered low or undetectable, between 110 and $200 \mathrm{pg} / \mathrm{ml}$ intermediate and levels $>200 \mathrm{pg} / \mathrm{ml}$ are con- 
sidered normal) [4]. The conventional treatment is symptomatic and includes stimulant drugs (amphetamine analogs and modafinil) targeting EDS and antidepressants to treat cataplexy [5]. Sodium oxybate (SO), also known as $\gamma$-hydroxybutyrate (GHB), is a new approach to treat all symptoms of the disease. It has been suggested that GHB has a role as a neurotransmitter or neuromodulator in the mammalian brain [6] and specific high-affinity binding sites have been identified in hippocampus, ventrolateral thalamus and the frontoparietal and entorhinal cortex of the rat brain [7].

GHB was first synthesized in 1960 and was shown to cross the blood-brain barrier rapidly to induce a sleeplike state with cardiovascular stability. In 1964, the substance was introduced as an intravenous anesthetic but was never largely accepted because of the high incidence of side effects (vomiting, seizures). In 1967, it was hypothesized that GHB might be beneficial for the treatment of sleep disorders [8]. Broughton and Mamelak [9, 10] decided to use GHB to normalize nighttime sleep in narcolepsy patients and thus to improve the daytime symptoms of the disease. These and following studies [11-13] demonstrated that the bedtime administration of GHB improved nighttime sleep but also daytime sleepiness, cataplexy, hypnagogic hallucinations and sleep paralysis. Parallel to these studies, reports appeared that GHB enhanced the effect of steroids and the release of growth hormone [14]. As a consequence, the drug was used by bodybuilders and for strength training. After the reports of a few fatal overdose cases in bodybuilders, GHB was banned by the American Food and Drug Administration (FDA) in 1990. This was followed by illicit use of GHB as a recreational drug because of its euphoric effects, disinhibition and sexual arousal. It was also implicated in a number of sexual assault cases and was labeled as 'date rape drug'. Especially in combination with alcohol GHB, causes anterograde amnesia and the victims are unable to recall any details of the event. In Switzerland, mainly cases with acute GHB intoxication have been reported [15-17]. According to the GHB factsheet, issued by the Swiss National Health Organization (Bundesamt für Gesundheit, BAG) between 1997 und 2005, 334 cases with GHB intoxication were reported to the Swiss Toxicological Information Centre, of which only 1 was fatal [18]. Recently, 3 fatal cases in patients with NC $(n=2)$ and sleep apnea $(n=1)$ treated with SO were reported [19], but the link with SO was not clear in any of them [20].

In 1994, Orphan Medical began the development of SO oral solution and after the safety and the efficacy of the drug for the treatment of narcolepsy were established in two multicenter, double-blind, placebo-controlled, randomized trials $[21,22]$, the FDA approved it for the treatment of cataplexy in narcolepsy in 2002 and for the treatment of EDS in 2005. In 2005, SO was also approved by the European Medicines Agency (EMEA). Swissmedic approved it in Switzerland in 2006. In the mean time, the long-term efficacy [23] and the effect of SO on daytime alertness have been shown [24-27]. Based on A-level evidence, SO has been recommended as a first-line treatment for cataplexy in $\mathrm{NC}$ by the European Federation of Neurological Societies (EFNS) Task Force [28].

In this study, we present our own experience in the treatment of NC patients with SO.

\section{Patients and Methods}

Eighteen HLA-DQB1*0602-positive (18/18), hypocretin-deficient (17/17) NC patients ( 9 men), mean age 43 years \pm 16 (SD), mean disease duration $14 \pm 14$ years, were treated with SO because of persistent/severe cataplexy $(\mathrm{n}=18 / 18)$ and EDS $(\mathrm{n}=$ 18/18). Disturbed nighttime sleep was reported in 17/18 patients. The Epworth sleepiness score (ESS), frequency of cataplexy, sleep paralysis, hallucinations and nightmares were assessed before and after treatment. Video-polysomnography (PSG) was performed in 17/18 patients before treatment and in 6/18 patients after treatment, MSLT in 16/18, respectively in 10/18, maintenance of wakefulness test (MWT) and actigraphy in 15/18, respectively in $10 / 18$, and steer-clear vigilance test (SCVT) in $14 / 18$, respectively in $10 / 18$ patients.

PSG consisted of four-channel EEG (C3/A2, C4/A1, O1/A2, O2/A1), left and right electrooculography (EOG), submental electromyography (EMG), electrocardiography (ECG), respiratory flow and effort, pulse oximetry and left and right anterior tibialis EMG. All recordings were done on Embla Somnologica ${ }^{\mathrm{TM}}$ Studio. Sleep stages, apneas/hypopneas, periodic limb movements and arousals were scored manually according to international criteria [2, 29-31]. Sleep onset was defined as the first epoch of either NREM-2 or REM sleep.

Standard MSLT [32] was performed on the day following the nocturnal recording, starting $2 \mathrm{~h}$ after waking up. For each patient, four or five naps with a duration of $20 \mathrm{~min}$ in 2-hour intervals were recorded. The naps were terminated after $20 \mathrm{~min}$. MWT was performed at daytime, for each patient four naps, each with a maximal duration of $40 \mathrm{~min}$ in 2-hour intervals were recorded. In case the patient fell asleep, the recording was interrupted after three consecutive epochs of NREM-1 or after one epoch of any other sleep stage. Sleep onset for both MSLT and MWT was defined as the first epoch of sleep.

Fourteen-day actigraphy was performed using Actiwatch ${ }^{\circledR}$ (Cambridge Neurotechnology); the main parameter of interest was the sleep/rest efficiency in percent. Vigilance during a monotonous task was assessed using the steer-clear vigilance test (SCVT) computer program [33]. For SCVT an arbitrary cut-off of 
$3 \%$ has been accepted in different sleep laboratories as normal controls usually lie clearly below this value [33].

Statistical analysis was performed using SPSS 15 software. $\chi^{2}$, paired $t$ tests and Wilcoxon signed rank tests were used to analyze categorical and continuous variables, respectively. Significance level was set at $\mathrm{p}<0.05$.

\section{Results}

All patients were HLA-DQB1*0602-positive and in $17 / 18$, CSF levels of hypocretin-1 were measured. It was undetectable in 13 , low in 3 , and intermediate in 1 patient. ESS was assessed in all patients at baseline. All patients reported cataplexy with a monthly frequency ranging form 1 to 600 (median 16, mean 101). Sleep paralysis was reported in 11/18 patients, hypnagogic hallucinations in 14/18, nightmares in 4/18 and disturbed nighttime sleep in 17/18 patients. The demographics, ESS, PSG, MSLT, MWT, actigraphy and SCVT data for all patients prior to treatment are shown in table 1.

\section{Patients on Sodium Oxybate}

Thirteen out of 18 patients are still treated with SO (7 men, mean dosage $6.4 \pm 1.2 \mathrm{~g}$, mean treatment duration $26 \pm 13$ months). Additional therapies include modafinil $(\mathrm{n}=5)$, fluoxetine $(\mathrm{n}=1)$ and methylphenidate $(\mathrm{n}=1)$. One patient stopped modafinil after the initiation of SO, in all the others the additional therapies remained stable.

One patient reported overall improvement of the narcolepsy symptoms of about $80 \%$ with one single bedtime dose of $5 \mathrm{~g}$ SO. Another 2 patients admitted skipping the second dose occasionally but in spite of that did not notice any significant difference in their symptoms. The remaining 10 patients reported taking SO regularly twice - at bedtime and between 2.5 and $4 \mathrm{~h}$ later.

Nearly all patients (12/13) reported subjective improvement in EDS (from 20 to $80 \%$, mean $33 \pm 23 \%$ ). ESS significantly improved (from 17.9 to $13.9, \mathrm{p}=0.001$ ). After treatment, $6 / 13$ patients were free of cataplexy, in 7/13 patients a marked reduction in cataplexy frequency, duration and severity was observed. Cataplexy under SO usually occurred later in the day, when the patients were tired. In general the improvement of cataplexy was highly significant (median before treatment 20 per month, after treatment $1, \mathrm{p}=0.001$ ). The reduction in percent was between 50 and 100\%, mean \pm SD, $88 \pm 19 \%$. Sleep paralyses disappeared in $8 / 8$ patients after initiation of $\mathrm{SO}$. Under SO treatment, 8/10 patients were free of hallucinations, 2 patients reported significant reduction in the frequency of hallucinations. Nightmares disappeared in 3

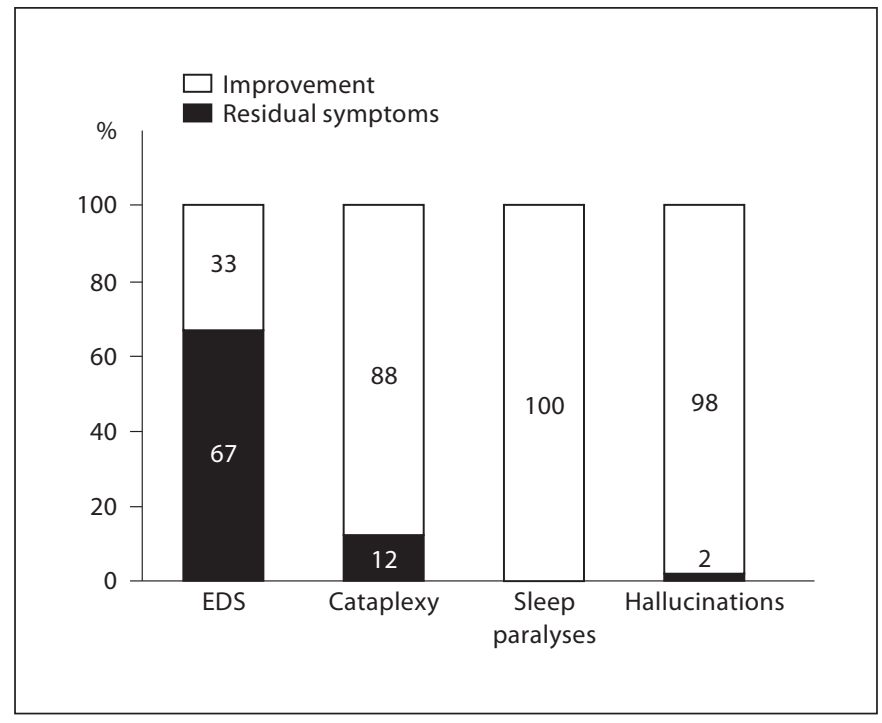

Fig. 1. Improvement of clinical symptoms after treatment, in percent.

Table 1. Characteristics of the patients prior to treatment with SO

\begin{tabular}{lcc}
\hline Variable & Mean \pm SD & Range \\
\hline Age, years & $43 \pm 16$ & $18-69$ \\
Male gender & 9 & \\
Disease duration, years & $14 \pm 14$ & $2-50$ \\
ESS & $17 \pm 4$ & $9-24$ \\
Cataplexy frequency per month, median & 16 & $1-600$ \\
Sleep paralysis per month & $5 \pm 9$ & $0-30$ \\
Hallucination frequency per month & $7 \pm 8$ & $0-30$ \\
Sleep/rest efficiency in actigraphy, \% & $62 \pm 19$ & $16-81$ \\
Rest/sleep for 24 h, \% & $37 \pm 12$ & $10-72$ \\
Sleep latency, min & $8 \pm 10$ & $0-38$ \\
Sleep efficiency, \% & $88 \pm 9$ & $71-98$ \\
REM latency, min & $35 \pm 62$ & $0-232$ \\
Apnea/hypopnea index/h & $11 \pm 18$ & $0-68$ \\
PLMS index/h & $15 \pm 24$ & $0-70$ \\
NREM-1, \% & $18 \pm 6$ & $9-31$ \\
NREM-2, \% & $36 \pm 10$ & $19-54$ \\
Slow wave sleep, \% & $12 \pm 7$ & $0-22$ \\
REM, \% & $21 \pm 8$ & $12-38$ \\
Arousal index/h & $16 \pm 7$ & $7-32$ \\
Mean sleep latency in MSLT & $1.8 \pm 1.2$ & $0.4-4.6$ \\
Number of SOREM in MSLT & $3.6 \pm 0.7$ & $2-4$ \\
Mean sleep latency in MWT & $6.3 \pm 4.1$ & $0.5-15.8$ \\
Number of SOREM in MWT & $1.1 \pm 1.2$ & $0-3$ \\
Error \% in SCVT & $8.5 \pm 9.7$ & $0.6-37.8$ \\
& & \\
\end{tabular}

ESS $=$ Epworth sleepiness score; MSLT = multiple sleep latency test; MWT = maintenance of wakefulness test; SOREM = sleep-onset REM period; SCVT = steer-clear vigilance test. 
Table 2. Effect of SO on different subjective and objective parameters

\begin{tabular}{|c|c|c|c|c|}
\hline Variable & Cases, $\mathrm{n}$ & $\begin{array}{l}\text { Before SO treatment } \\
\text { mean } \pm S D\end{array}$ & $\begin{array}{l}\text { After SO treatment } \\
\text { mean } \pm S D\end{array}$ & $\mathrm{p}$ value \\
\hline ESS & 13 & $17.8 \pm 1$ & $13.9 \pm 1.2$ & 0.001 \\
\hline Number of cataplexies/month, median & 13 & 20 & 1 & 0.001 \\
\hline Number of sleep paralyses/month & 13 & $4.1 \pm 8.3$ & 0 & 0.011 \\
\hline Number of hallucinations/month & 13 & $7.6 \pm 9.5$ & $0.4 \pm 1.1$ & 0.005 \\
\hline Nightmares/month & 13 & $6.5 \pm 10.3$ & $1.5 \pm 5.5$ & NS \\
\hline Sleep/rest efficiency in actigraphy & 8 & $60.5 \pm 19.7$ & $76 \pm 4$ & 0.017 \\
\hline Mean sleep latency in MSLT, min & 8 & $2 \pm 1.5$ & $2.4 \pm 2.6$ & NS \\
\hline Number of SOREM in MSLT & 8 & $3.6 \pm 0.5$ & $2.4 \pm 1.4$ & 0.038 \\
\hline Mean sleep latency in MWT, min & 9 & $5.5 \pm 4.5$ & $17.4 \pm 8.9$ & $<0.001$ \\
\hline Number of SOREM in MWT & 9 & $1 \pm 1.1$ & $0.4 \pm 1.1$ & NS \\
\hline Error \% in SCVT & 8 & $10.8 \pm 12.5$ & $2.1 \pm 2.1$ & 0.017 \\
\hline Total sleep time in PSG, min & 5 & $390 \pm 50$ & $398 \pm 42$ & NS \\
\hline Sleep latency in PSG, min & 5 & $11.7 \pm 16$ & $5.6 \pm 7.5$ & NS \\
\hline Sleep efficiency in PSG, \% & 5 & $88.5 \pm 7.9$ & $92.1 \pm 5.7$ & NS \\
\hline REM latency, min & 5 & $76.6 \pm 109.4$ & $21.6 \pm 41.9$ & NS \\
\hline NREM-1, \% & 5 & $19.1 \pm 5.5$ & $8.2 \pm 2.8$ & 0.071 \\
\hline NREM- $2, \%$ & 5 & $38.3 \pm 10.2$ & $37.5 \pm 9.3$ & NS \\
\hline Slow wave sleep, $\%$ & 5 & $10.5 \pm 7.7$ & $33.1 \pm 14.7$ & 0.074 \\
\hline Apnea/hypopnea index/h & 5 & $22.4 \pm 27.8$ & $16.2 \pm 16.7$ & NS \\
\hline PLMS index/h & 5 & $7.1 \pm 7.2$ & $8.3 \pm 14.8$ & NS \\
\hline Arousal index & 5 & $20.5 \pm 6.3$ & $9.2 \pm 2.3$ & 0.067 \\
\hline
\end{tabular}

and persisted in 1 of 4 patients. All patients experienced a subjective improvement of their nighttime sleep. The improvement of the clinical symptoms of NC is presented in figure 1.

Rest/sleep efficiency in the actigraphy improved (from 61 to $76 \%, p=0.017$ ), the mean sleep latency in MSLT did not differ significantly, the mean sleep latency in MWT increased clearly (from 5.5 to $17.4 \mathrm{~min}, \mathrm{p}<0.001$ ) and the error quotient in SCVT decreased (from 10.7 to $2.1 \%$, $\mathrm{p}=$ 0.017). In PSG a decrease in sleep latency, REM sleep latency, apnea/hypopnea index, NREM-1, REM and arousal index and an increase in slow wave sleep was observed, although as PSG was available before and after treatment in only 5 patients, the results did not reach statistical significance. The results of the different tests are presented in table 2 . An actigraphy and a PSG hypnogram example before and after treatment are shown in figure 2 .

\section{Dropouts}

In 5 patients the drug was discontinued shortly after initiation due to insufficient compliance $(\mathrm{n}=2)$, lack of efficiency $(n=2)$ or side effects $(n=1)$, including nausea and worsening of sleepiness and cataplexy. This patient did not tolerate modafinil and fluoxetine either and did not wish further treatment. Four of these patients had rare cataplexy (once or twice a month); 3 were treated with modafinil (as monotherapy 400-600 mg/day, or together ( $200 \mathrm{mg} /$ day) with venlafaxine $75 \mathrm{mg} /$ day or fluoxetine $20 \mathrm{mg} /$ day). One patient was treated with clomipramine $100 \mathrm{mg} /$ day. In these patients no follow-up assessment under SO was possible as they discontinued the medication shortly after initiation (1 week in most cases).

\section{Adverse Events}

The most common adverse event (considering all 18 patients) was nighttime confusion $(\mathrm{n}=6)$. Others included nausea $(n=2)$, enuresis ( $n=2$, in 1 of the cases only once over 1.5 years), diffuse muscle pain, headache, flatulence, disturbance in attention and forgetfulness ( $\mathrm{n}=$ 1). The patient with diffuse muscle pain also had enuresis; after a dose reduction from 6 to $4.5 \mathrm{~g} /$ day the adverse events decreased and the patient insisted on taking the medication. Only 1 patient discontinued treatment because of adverse events. This patient reported pro- 


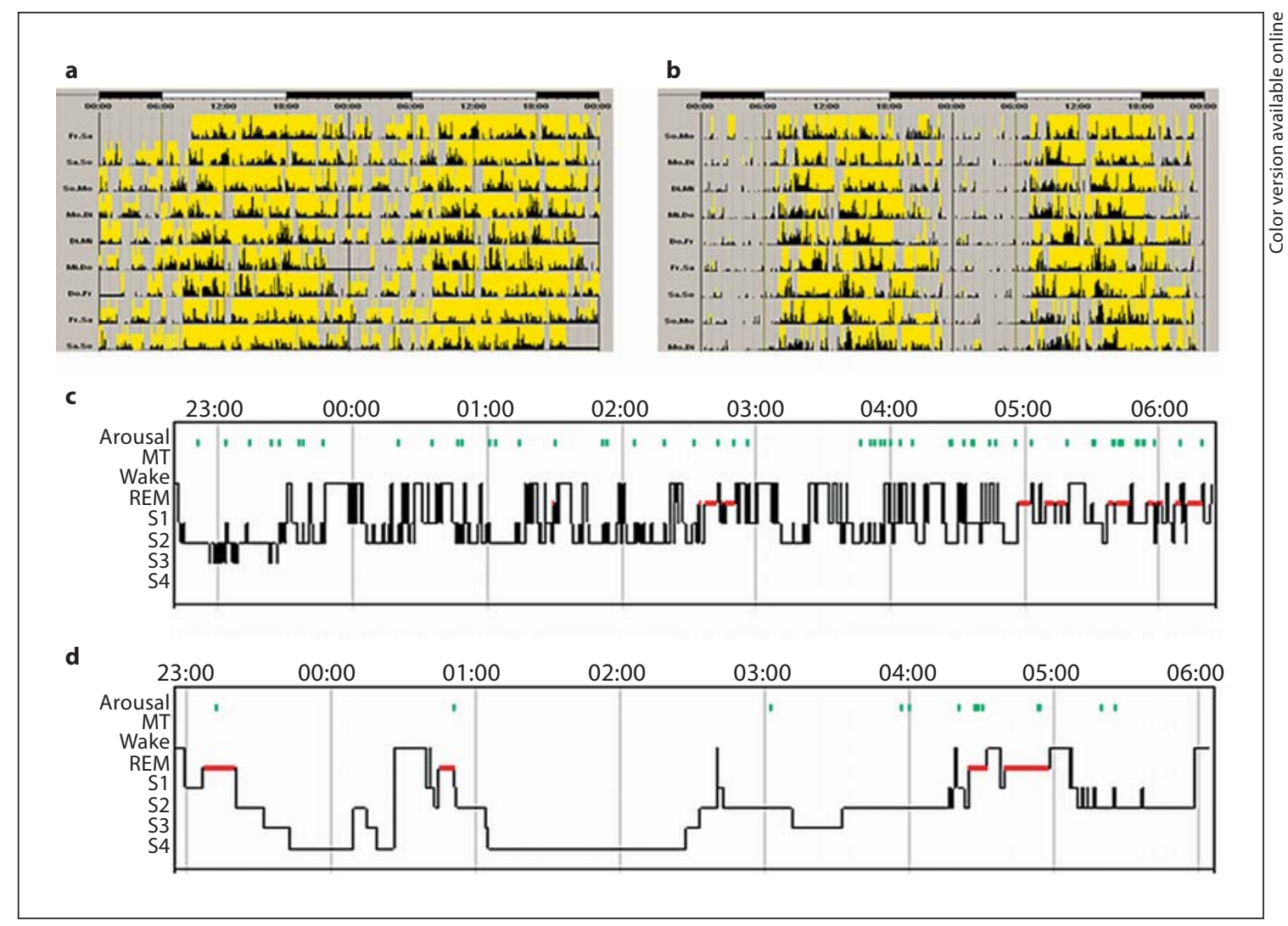

Fig. 2. Actigraphy and PSG hypnogram examples before and after treatment with SO: a actigraphy before, b actigraphy after, $\mathbf{c}$ hypnogram before, and $\mathbf{d}$ hypnogram after treatment.

nounced nausea and worsening of nighttime sleep and cataplexy.

There was 1 patient who over time voluntarily increased the dose of SO from $2 \times 4.5 \mathrm{~g}$ to $3 \times 4.5 \mathrm{~g}$ per night because of nighttime sleep disturbance.

\section{Discussion}

\section{Efficacy: Clinical Findings}

SO led to significant improvement of cataplexy, daytime sleepiness and alertness, sleep paralysis, hallucinations, nightmares and nighttime sleep. Actigraphy and SCVT were used for the first time to assess the treatment effects of SO.

Cataplexy improvement regarding its frequency, duration and severity has been shown in a number of studies $[10-12,21-27,34]$. As already reported by Mamelak et al. [34], cataplexy under SO occurred later in the day, when the patients were tired. Consistent with previous studies
$[11,21,24,26,27,34]$, improvement of daytime sleepiness and alertness was demonstrated. As 5 patients were treated with modafinil (in 1 in alternation with methylphenidate) and 1 with fluoxetine, the effects on EDS cannot only be attributed to SO. Sleep paralysis and hallucinations improved too, as previously reported [11-13, 34]. In more recent studies [21,23-25], a decrease in the frequency of sleep paralyses and hallucinations was observed, but the results did not reach statistical significance. Nightmares disappeared in 3 of our patients, 1 of them reported that she continued dreaming but the dreams were no longer frightening. This phenomenon has also been described by Mamelak et al. [34]. A literature overview of different studies, assessing the effects of SO, is presented in table 3.

Efficacy: Actigraphy, MSLT, MWT, SCVT and PSG

Actigraphy showed a marked improvement in the nighttime rest/sleep efficiency, consistent with the subjectively reported improvement of nighttime sleep. Simi- 
Table 3. Literature overview

\begin{tabular}{|c|c|c|c|c|c|c|c|c|}
\hline $\begin{array}{l}\text { Reference } \\
\text { (first author) }\end{array}$ & Design & $\begin{array}{l}\text { Patients } \\
\mathrm{n}\end{array}$ & Methods & $\begin{array}{l}\text { EDS/sleep } \\
\text { attacks }\end{array}$ & Cataplexy & $\begin{array}{l}\text { Sleep } \\
\text { paralyses }\end{array}$ & $\begin{array}{l}\text { Hallucina- } \\
\text { tions }\end{array}$ & $\begin{array}{l}\text { Nighttime } \\
\text { sleep quality }\end{array}$ \\
\hline $\begin{array}{l}\text { Broughton } \\
1979[10]\end{array}$ & case series, open & 16 & clinical assessment & improved & improved & NA & NA & improved \\
\hline $\begin{array}{l}\text { Scharf } \\
1985[11]\end{array}$ & case series, open & 30 & $\begin{array}{l}\text { clinical assessment, } \\
\text { PSG }\end{array}$ & improved & improved & improved & improved & improved \\
\hline $\begin{array}{l}\text { Mamelak } \\
1986[34]\end{array}$ & case series, open & 48 & $\begin{array}{l}\text { clinical assessment, } \\
\text { PSG, MSLT }\end{array}$ & improved & improved & improved & improved & improved \\
\hline $\begin{array}{l}\text { Scrima } \\
1989[12]\end{array}$ & $\begin{array}{l}\text { double-blind, } \\
\text { counter-balanced } \\
\text { crossover design }\end{array}$ & 20 & $\begin{array}{l}\text { clinical assessment, } \\
\text { daily diary }\end{array}$ & $\begin{array}{l}\text { not } \\
\text { significantly } \\
\text { different }\end{array}$ & improved & NA & NA & improved \\
\hline $\begin{array}{l}\text { Lammers } \\
1993[13]\end{array}$ & $\begin{array}{l}\text { randomized, } \\
\text { double-blind } \\
\text { placebo-controlled } \\
\text { crossover design }\end{array}$ & 24 & $\begin{array}{l}\text { daily diary, } \\
\text { questionnaires, } \\
\text { PSG, MSLT }\end{array}$ & improved & $\begin{array}{l}\text { improved, } \\
\text { but not } \\
\text { significantly }\end{array}$ & $\begin{array}{l}\text { low } \\
\text { incidence, } \\
\text { not accessible }\end{array}$ & improved & $\begin{array}{l}\text { improved, } \\
\text { but not } \\
\text { significantly }\end{array}$ \\
\hline $\begin{array}{l}\text { US Xyrem }^{\circledR} \\
\text { Multicenter } \\
\text { Study Group } \\
2002 \text { [21] }\end{array}$ & $\begin{array}{l}\text { multicenter, } \\
\text { double-blind } \\
\text { placebo-controlled }\end{array}$ & 136 & $\begin{array}{l}\text { daily diary, } \\
\text { questionnaires }\end{array}$ & $\begin{array}{l}\text { improved, } \\
\text { dose- } \\
\text { dependent }\end{array}$ & $\begin{array}{l}\text { improved, } \\
\text { dose- } \\
\text { dependent }\end{array}$ & $\begin{array}{l}\text { improved, } \\
\text { but not } \\
\text { significantly }\end{array}$ & $\begin{array}{l}\text { improved, } \\
\text { but not } \\
\text { significantly }\end{array}$ & $\begin{array}{l}\text { improved, } \\
\text { dose- } \\
\text { dependent }\end{array}$ \\
\hline $\begin{array}{l}\text { US Xyrem }^{\circledR} \\
\text { Multicenter Study } \\
\text { Group } 2003 \text { [23] }\end{array}$ & open-label & 118 & $\begin{array}{l}\text { daily diary, } \\
\text { questionnaires }\end{array}$ & $\begin{array}{l}\text { long-term } \\
\text { improvement }\end{array}$ & $\begin{array}{l}\text { long-term } \\
\text { improve- } \\
\text { ment }\end{array}$ & $\begin{array}{l}\text { improved, } \\
\text { but not } \\
\text { significantly }\end{array}$ & $\begin{array}{l}\text { improved, } \\
\text { but not } \\
\text { significantly }\end{array}$ & $\begin{array}{l}\text { improved, } \\
\text { but not } \\
\text { significantly }\end{array}$ \\
\hline $\begin{array}{l}\text { Mamelak } \\
2004[24]\end{array}$ & open-label study & 25 & $\begin{array}{l}\text { PSG, MWT, } \\
\text { questionnaire }\end{array}$ & improved & improved & improved & improved & improved \\
\hline $\begin{array}{l}\text { Xyrem }^{\circledR} \\
\text { International } \\
\text { Study Group } \\
2005 \text { [25] }\end{array}$ & $\begin{array}{l}\text { double-blind } \\
\text { placebo-controlled }\end{array}$ & 228 & daily diary & NA & $\begin{array}{l}\text { improved, } \\
\text { dose- } \\
\text { dependent }\end{array}$ & $\begin{array}{l}\text { improved, } \\
\text { but not } \\
\text { significantly }\end{array}$ & $\begin{array}{l}\text { improved, } \\
\text { but not } \\
\text { significantly }\end{array}$ & NA \\
\hline $\begin{array}{l}\text { Xyrem }^{\circledR} \\
\text { International } \\
\text { Study Group } \\
2005 \text { [27] }\end{array}$ & $\begin{array}{l}\text { double-blind } \\
\text { placebo-controlled }\end{array}$ & 228 & $\begin{array}{l}\text { daily diary, } \\
\text { questionnaires, } \\
\text { MWT }\end{array}$ & $\begin{array}{l}\text { improved, } \\
\text { dose- } \\
\text { dependent }\end{array}$ & NA & NA & NA & NA \\
\hline $\begin{array}{l}\text { Black } \\
2006[26]\end{array}$ & $\begin{array}{l}\text { multicenter, } \\
\text { double-blind } \\
\text { placebo-controlled }\end{array}$ & 270 & $\begin{array}{l}\text { daily diary, } \\
\text { questionnaires, } \\
\text { MWT }\end{array}$ & improved & NA & NA & NA & NA \\
\hline $\begin{array}{l}\text { Black } \\
2009[36]\end{array}$ & $\begin{array}{l}\text { double-blind } \\
\text { placebo-controlled }\end{array}$ & 278 & $\begin{array}{l}\text { daily diary, } \\
\text { questionnaires, } \\
\text { PSG, MWT }\end{array}$ & improved & NA & NA & NA & improved \\
\hline
\end{tabular}

MSLT = Multiple sleep latency test; MWT = maintenance of wakefulness test; NA = not available; PSG = polysomnography.

larly, a significant subjective nighttime sleep improvement $[11-13,34]$ and a significant decrease in the number of nighttime awakenings have been reported [21, 25]. To our knowledge, actigraphy data has not been previously used to assess the treatment effects of SO. Actigraphy allows the assessment of sleep-wake patterns over longer periods of time. As seen in figure 2, the results are comparable with the hypnogram, obtained after an overnight PSG. We suggest that the method can be a useful, far less expensive and less complex tool in the evaluation of the treatment effects of $\mathrm{SO}$, especially concerning nighttime sleep improvement.

There were no significant differences in daytime sleep latency in MSLT as already reported in the literature [34]. Concordant with previous studies, a significant improvement of the mean sleep latency in MWT was observed $[24,26,27]$. The SCVT demonstrated an improvement in sustained attention. This result can be compared to the 
improved ability to stay awake in MWT, but whereas MWT requires a whole day of testing, SCVT is completed in $30 \mathrm{~min}$. Additionally, it is in a way closer to some everyday life situations, where patients are involved in monotonous tasks, but their active participation is still required. We suggest that the test is used complementary to MWT.

SO also leads to changes in nighttime sleep architecture, such as decrease of NREM-1 [24], increase of slow wave sleep $[24,34]$ and decrease in nighttime awakenings $[21,24]$. As PSG was available in only 5 patients before and after treatment, we could not find significant differences in nighttime sleep parameters. Nevertheless, a trend for a decrease in NREM-1 and in the arousal index and a trend for increase in slow wave sleep were observed. Although not significant, there was a decrease in apnea/ hypopnea index after SO treatment. This finding is in line with a recent report in which the use of SO $4.5 \mathrm{~g} /$ night over 2 weeks did not generate respiratory depressant effects in obstructive sleep apnea patients as measured by the apnea/hypopnea index, obstructive apnea events, central apneas, and oxygen saturation [35].
Safety

SO was well tolerated in most patients. Consistent with the literature $[21,23,25]$, adverse events primarily affected the central nervous system and digestive system. In most patients, there was no dose relationship for the most common adverse events and the majority were mild to moderate in severity. In 1 patient, dose reduction led to improvement of the adverse events. Only 1 patient discontinued the medication because of nausea and worsening of the narcolepsy symptoms.

\section{Conclusion}

SO is effective not only for cataplexy but also for EDS, sleep paralyses, hallucinations and nighttime sleep quality in NC. The drug is generally well tolerated in spite of the occurrence of mild-to-moderate adverse events. Actigraphy offers a cheaper and less complex alternative to PSG for assessment of SO treatment effects, especially in everyday practice, and vigilance tests should be used complementary to MWT in order to assess sustained attention.

\section{References}

1 Bassetti C, Aldrich MS: Narcolepsy. Neurol Clin 1996;14:545-571

2 American Academy of Sleep Medicine (AASM): International Classification of Sleep Disorders. Diagnostic and Coding Manual, ed 2. Westchester/IL, AASM, 2005.

3 Lin L, Hungs M, Mignot E: Narcolepsy and the HLA region. J Neuroimmunol 2001;117: 9-20.

4 Mignot E, Lammers GJ, Ripley B, et al: The role of cerebrospinal fluid hypocretin measurement in the diagnosis of narcolepsy and other hypersomnias. Arch Neurol 2002;59: 1553-1562.

5 Billiard M: Narcolepsy: current treatment options and future approaches. Neuropsychiatr Dis Treat 2008;4:557-666.

6 Vayer P, Mandel P, Maitre M: $\gamma$-Hydroxybutyrate, a possible neurotransmitter. Life Sci 1987;41:1547-1557.

7 Snead OC, Liu C: $\gamma$-Hydroxybutyric acid binding sites in rat and human brain synaptosomal membranes. Biochem Pharmacol 1984;33:2587-2590.

8 Yamada Y, Yamamoto J, Fujiki A, Hishakawa Y, Kaneko Z: Effect of butyrolactone and $\gamma$ hydroxybutyrate on the EEG and sleep cycle in man. Electroencephalogr Clin Neurophysiol 1967;22:558-562.
9 Broughton R, Mamelak M: In Guilleminault C, Dement WC, Passousant P (eds): Advances in Sleep Research. New York, Spectrum, 1976, pp 659-667.

10 Broughton R, Mamelak M: The treatment of narcolepsy-cataplexy with nocturnal $\gamma$-hydroxybutyrate. Can J Neurol Sci 1979;6:1-6.

11 Scharf M, Brown D, Woods M, et al: The effects and effectiveness of $\gamma$-hydroxybutyrate in patients with narcolepsy. J Clin Psychiatry $1985 ; 46: 222-225$

12 Scrima L, Hartman PG, Johnson FH Jr, Hiller FC: Efficacy of $\boldsymbol{\gamma}$-hydroxybutyrate versus placebo in treating narcolepsy-cataplexy: double-blind subjective measures. Biol Psychiatry 1989;26:331-343.

13 Lammers GJ, Arends J, Declerck AC, et al $\gamma$-Hydroxybutyrate and narcolepsy: a double-blind placebo-controlled study. Sleep 1993;6:216-220.

14 Takahara J, Younuki S, Yakushiji W: Stimulatory effects of $\gamma$-hydroxybutyric acid on grow th hormone and prolactin release in humans. J Clin Endocrinol Metab 1977;44: 1014-1016.

15 Herbrand R, Greminger P, Schurter G, Pletscher W, Kupferschmidt H: Sudden coma in a 20-year-old man (in German). Praxis (Bern 1994) 2000;89:1326-1330.
16 Liechti ME, Kupferschmidt H: $\gamma$-Hydroxybutyrate $(\mathrm{GHB})$ and $\boldsymbol{\gamma}$-butyrolactone $(\mathrm{GBL})$ : analysis of overdose cases reported to the Swiss Toxicological Information Centre. Swiss Med Wkly 2004;134:534-537.

17 Iten PX, Oestreich A, Lips R, Brabetz M: A new drug reaches Switzerland: coma after intake of $\gamma$-hydroxybutyrate (GHB) (in German). Schweiz Med Wochenschr 2000;130: 356-361.

18 Bundesamt für Gesundheit BAG. Factsheet GHB 2009

19 Zvosec DL, Smith SW, Hall BJ: Three deaths associated with use of Xyrem ${ }^{\circledR}$. Sleep Med 2009; 10:490-493.

20 Lammers GJ, Bassetti C, Billiard M et al: Sodium oxybate is an effective and safe treatment for narcolepsy. Sleep Med 2010;11:105106.

21 US Xyrem ${ }^{\circledR}$ Multicenter Study Group: A randomized, double-blind, placebo-controlled multi-center trial comparing the effects of three doses of orally administered sodium oxybate with placebo for the treatment of narcolepsy. Sleep 2002;25:42-49.

22 US Xyrem ${ }^{\circledR}$ Multicenter Study Group: Sodium oxybate demonstrates long-term efficacy for the treatment of cataplexy in patients with narcolepsy. Sleep Med 2004;5:119-123. 
23 US Xyrem ${ }^{\circledR}$ Multicenter Study Group: A 12-month, open-label, multicenter extension trial of orally administered sodium oxybate for the treatment of narcolepsy. Sleep 2003;1: 31-35.

24 Mamelak M, Black J, Montplaisir J, Ristanovic R: A pilot study on the effects of sodium oxybate on sleep architecture and daytime alertness in narcolepsy. Sleep 2004;27: 1327-1334.

25 Xyrem ${ }^{\circledR}$ International Study Group: Further evidence supporting the use of sodium oxybate for the treatment of cataplexy: a doubleblind, placebo-controlled study in 228 patients. Sleep Med 2005;6:415-421.

26 Black J, Houghton WC: Sodium oxybate improves excessive daytime sleepiness in narcolepsy. Sleep 2006;29:939-946.

27 Xyrem International Study Group: A doubleblind, placebo-controlled study demonstrates sodium oxybate is effective for the treatment of excessive daytime sleepiness in narcolepsy. J Clin Sleep Med 2005;1:391-397.
28 Billiard M, Bassetti C, Dauvilliers Y, et al EFNS guidelines on management of narcolepsy. Eur J Neurol 2006;13:1035-1048.

29 Rechtschaffen A, Kales A: A Manual of Standardized Terminology, Techniques and Scoring System for Sleep Stages of human Subjects. Los Angeles, UCLA Brain Information Service/Brain Research Institute, 1968.

30 American Sleep Disorders Association: EEG arousals: scoring rules and examples. A preliminary report from the Sleep Disorders Atlas Task Force of the American Sleep Disorders Association. Sleep 1992;15:174-184.

31 American Academy of Sleep Medicine Task Force: Sleep-disordered breathing disorders in adults: recommendations for syndrome definition and measurement techniques in clinical research. Sleep 1999;22;667-688.
32 Carskadon MA, Dement WC, Mitler MM, Roth T, Westbrook PR, Keenan S: Guidelines for the multiple sleep latency test (MSLT): a standard measure of sleepiness. Sleep 1986; 9:519-524.

33 Findley L, Unverzagt M, Guchu R, Fabrizio M, Buckner J, Suratt P: Vigilance and automobile accidents in patients with sleep apnea or narcolepsy. Chest 1995;108:619-624.

34 Mamelak M, Scharf MB, Woods M: Treatment of narcolepsy with $\gamma$-hydroxybutyrate. A review of clinical and sleep laboratory findings. Sleep 1986;9:285-289.

35 George CF, Feldman N, Zheng Y, Steininger TL, Grzeschik SM, Lai C, Inhaber N: A 2-week, polysomnographic, safety study of sodium oxybate in obstructive sleep apnea syndrome. Sleep Breath (in press).

36 Black J, Pardi D, Hornfeldt CS, Inhaber N: The nightly administration of sodium oxybate results in significant reduction in the nocturnal sleep disruption of patients with narcolepsy. Sleep Med 2009;10:829-835. 


\section{Neurology}

\section{Modafinil ameliorates excessive daytime sleepiness after traumatic brain injury \\ P.R. Kaiser, P.O. Valko, E. Werth, et al. \\ Neurology 2010;75;1780-1785 \\ DOI 10.1212/WNL.0b013e3181fd62a2}

This information is current as of November 15, 2010

The online version of this article, along with updated information and services, is located on the World Wide Web at:

http://www.neurology.org/content/75/20/1780.full.html

Neurology $®$ is the official journal of the American Academy of Neurology. Published continuously since 1951, it is now a weekly with 48 issues per year. Copyright Copyright (C) 2010 by AAN

Enterprises, Inc.. All rights reserved. Print ISSN: 0028-3878. Online ISSN: 1526-632X.

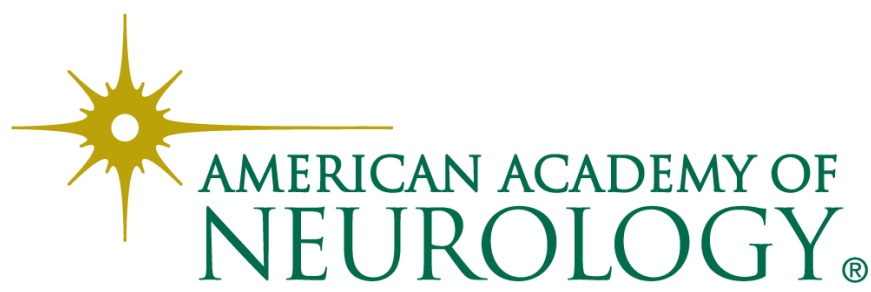


P.R. Kaiser, MSc*

P.O. Valko, $\mathrm{MD}^{*}$

E. Werth, PhD

J. Thomann, MSc

J. Meier

R. Stocker, MD

C.L. Bassetti, MD

C.R. Baumann, MD

Address correspondence and reprint requests to Dr. Christian R. Baumann, Department of Neurology, University Hospital Zurich, Frauenklinikstrasse 26, 8091 Zurich, Switzerland christian.baumann@usz.ch

\section{Modafinil ameliorates excessive daytime sleepiness after traumatic brain injury}

吅 $\underline{\underline{\underline{\underline{\underline{A}}}}}$

\section{ABSTRACT}

Background: Excessive daytime sleepiness (EDS) and fatigue are common symptoms after traumatic brain injury (TBI), but there is no specific treatment for affected patients. With this pilot study, we aimed at studying the effect of daily modafinil on posttraumatic EDS and fatigue.

Methods: We conducted a prospective, double-blind, randomized, placebo-controlled pilot study in 20 patients with TBI who had fatigue or EDS or both. After baseline examinations (questionnaires including the Epworth Sleepiness Scale to assess EDS and the Fatigue Severity Scale to assess fatigue, actigraphy, polysomnography, maintenance of wakefulness test, and psychomotor vigilance test), 10 patients received 100 to 200 mg modafinil every morning, and 10 patients were treated with placebo. After a 6-week treatment period, all examinations were repeated.

Results: EDS improved significantly in patients with TBI who were treated with modafinil, compared with the placebo group. Similarly, the ability to stay awake on the maintenance of wakefulness test improved only in the modafinil group. Modafinil, however, had no impact on posttraumatic fatigue. Clinically relevant side effects were not observed.

Conclusion: This study indicates that modafinil is effective and well tolerated in the treatment of posttraumatic EDS but not of fatigue.

Classification of evidence: This study provides Class I evidence that modafinil (100-200 mg daily) improves posttraumatic EDS compared with placebo. This study provides Class I evidence that modafinil (100-200 mg daily) does not improve posttraumatic fatigue compared with placebo. Neurology ${ }^{\circledR} 2010 ; 75: 1780-1785$

GLOSSARY

EDS = excessive daytime sleepiness; ESS = Epworth Sleepiness Scale; FSS = Fatigue Severity Scale; GCS = Glasgow Coma Scale; MWT = maintenance of wakefulness test; TBI = traumatic brain injury.

Traumatic brain injury (TBI) occurs in about 1.4 million people in the United States each year, corresponding to an incidence of 460 per 100,000 per year. ${ }^{1}$ Another study estimated that 3.17 million US citizens (more than 1\% of the total US population) have long-term neurologic disability that resulted from TBI. ${ }^{2}$ Thus, TBI causes the largest number of disability-adjusted life-years lost to death and to varying degrees of disability, including posttraumatic sleep-wake disturbances. ${ }^{3}$

Excessive daytime sleepiness (EDS) and fatigue are frequent symptoms after TBI. In our prospective study on posttraumatic sleep-wake disturbances, we studied 65 consecutive patients by means of questionnaires and sleep laboratory examinations 6 months after trauma. ${ }^{4}$ We found EDS in $38 \%$ and fatigue in $17 \%$ of our patients. Our findings are in line with other reports on posttraumatic sleep-wake disturbances. ${ }^{5-7}$ The etiology of posttraumatic EDS is not known. Recently, we found a partial loss of wake-promoting hypothalamic hypocretin (orexin) neurons after fatal TBI. ${ }^{8}$ EDS and fatigue significantly impair quality of life and daytime functioning, including professional performances and social activities. ${ }^{4}$ Despite their high inci-

\footnotetext{
*The first 2 authors contributed equally to this study.

From the Departments of Neurology (P.R.K., P.O.V., E.W., J.T., J.M., C.L.B., C.R.B.) and Surgical Intensive Care (R.S.), University Hospital Zurich; and Department of Neurology (C.L.B.), Neurocenter of Southern Switzerland, Ospedale Civico, Lugano, Switzerland.

Study funding: Supported by Schweizerischer Versicherungsverband (SVV), Zurich, Switzerland, and Cephalon GmbH, Martinsried, Germany.

Disclosure: Author disclosures are provided at the end of the article.
} 
dences, posttraumatic EDS and fatigue are still underestimated, and there is no treatment for affected patients.

Thus, with this pilot study, we aimed at studying the effect of daily modafinil on posttraumatic EDS and fatigue. Modafinil (2[(diphenylmethyl) sulfinyl] acetamide) has wake-promoting properties and is approved by the US Food and Drug Administration for the treatment of EDS in patients with narcolepsy, obstructive sleep apnea syndrome, and shift work sleep disorder.

METHODS This prospective study was conducted at the Department of Neurology of the University Hospital of Zurich, Switzerland, between January 2007 and June 2009.

Subjects. In this prospective, double-blind, randomized, placebo-controlled pilot study, we included 20 consecutive patients from our first study on posttraumatic sleep-wake disturbances. ${ }^{4}$ All patients were admitted for closed mild to severe TBI to our surgical intensive care unit, where they were recruited for this study. TBI severity was quantified with the initial Glasgow Coma Scale (GCS) examination, which was performed immediately after trauma (mild TBI: GCS 13-15, moderate TBI: GCS 9-12, severe TBI: GCS 3-8). Admission to the surgical intensive care unit was for mild to severe TBI. The mandatory inclusion criterion was the presence of fatigue or EDS or both since TBI. Patients with neurologic, psychiatric, or other disorders and medication that might cause sleep-wake disturbances were not included. Furthermore, we excluded patients who revealed significant sleep-wake disturbances other than posttraumatic vigilance impairment at baseline examinations. Patients with chronic sleep deprivation were also excluded. The latter was diagnosed in patients with EDS who slept $\geq 2$ hours more on weekends or during holidays than during weekdays. All exclusions were made before randomization.

Fatigue was assessed with the Fatigue Severity Scale (FSS), and EDS was assessed with the Epworth Sleepiness Scale (ESS). Both scales have been validated in German. ${ }^{9} 10$ Fatigue is diagnosed in patients with FSS values above 4.0, and EDS is diagnosed in those with ESS scores $\geq 10$. Before randomization, written informed consent was obtained from all patients.

Baseline examinations. At baseline, patients were administered the Beck Depression and Anxiety Inventory, a standard questionnaire to assess depression and anxiety symptoms. ${ }^{11}$ To rule out chronic sleep deprivation, patients were examined by 2 weeks of actigraphy studies. Actigraphy is a noninvasive method to monitor activity/rest cycles over a defined period of time. Sleep and physical activity levels were recorded by wrist actigraphy. The actimeter includes a light sensor and is worn on the nondominant wrist (Actiwatch, CamNtech Ltd., Cambridge, UK). ${ }^{12}$ With this software, the determination of time asleep and time awake relies on an algorithm using the activity data recorded by the Actiwatch in a series of linked calculations. During actigraphy recordings, all patients simultaneously filled in sleep logs.

Immediately after actigraphy, conventional nocturnal polysomnography was recorded from $11 \mathrm{PM}$ to $7 \mathrm{AM}$. We used a 16-channel recording system (Embla A10, Embla, Broomfield,
CO). Sleep-stage scoring was performed visually by the first authors according to standard criteria. ${ }^{13}$ Patients with significant sleep-wake disturbances, such as obstructive sleep apnea, detected during polysomnographic recordings were excluded from the study. The day after polysomnography, all patients were examined by a maintenance of wakefulness test (MWT) ${ }^{14}$ This test measures the ability to stay awake for 40 minutes while sitting quietly in a dark room and is performed every 2 hours, in total 4 times a day. Mean sleep latency in the MWT was defined as the time from lights off to the first epoch of any sleep stage. At the same day, before every MWT test, we examined the patients' vigilance with the psychomotor vigilance test. This test measures reaction times of patients to the sudden appearance of a timekeeper on a black computer screen. ${ }^{15}$ The test lasts 10 minutes and assesses the number of lapses, defined as times the subject does not respond to the signal in time, and calculates reaction times. Intake of caffeine and medication with known effects on vigilance and sleepiness were not allowed during days and nights with sleep recordings and vigilance tests.

Randomization and follow-up examinations. After completion and evaluation of all tests, included patients were randomly assigned to either the modafinil group or the placebo group. All study procedures were performed in a strict doubleblind manner. For allocation concealment, labeling of masked study medication (modafinil or placebo) by manufacturing of numbered containers was performed centrally by the Pharmacy of the University Hospital of Zurich. All patients in the treatment group started with 1 oral capsule (corresponding to 100 $\mathrm{mg}$ modafinil per day in the treatment group), administered in the morning. Every 2 weeks, a phone call from the first author evaluated the subjective response to treatment, including efficacy and side effects. In patients without clear efficacy and without side effects, the dose was doubled: 1 oral capsule in the morning and 1 at noon. In patients with troublesome side effects with 2 capsules, there was a possibility to reduce the dose again. Furthermore, all patients were asked whether they estimated their vigilance impairment to be ameliorated by treatment.

After 6 treatment weeks, all examinations, including questionnaires, actigraphy, conventional nocturnal polysomnography, MWTs, and psychomotor vigilance tests, were repeated. Primary outcome measures included improvements in FSS and ESS values, and in mean sleep latencies on MWT.

Standard protocol approvals, registrations, and patient consents. The study was approved by the local ethical standards committee on human experimentation (Ethikkommission des Kantons Zurich) and by Swissmedic, the Swiss agency for therapeutic products. All study participants gave oral and written consent to all study procedures.

Statistics. Statistical analyses were performed by using correlation analyses (Pearson and Spearman), Student $t$ tests, Mann-Whitney $U$ tests, and multivariate regression analyses. Parametric tests were 2-tailed.

RESULTS Demographic data and baseline characteristics of both the treatment and the placebo group can be found in table 1. Patients in both groups did not differ in age, sex distribution, severity of TBI, or duration since trauma. At study inclusion, the duration since trauma was 1 to 3 years in all patients. After baseline examinations, we excluded 4 patients because of obstructive sleep apnea and 1 patient be- 


\begin{tabular}{|c|c|c|c|c|}
\hline \multirow[t]{2}{*}{ Table 1} & \multicolumn{4}{|c|}{$\begin{array}{l}\text { Baseline characteristics and changes from baseline in } 20 \text { patients } \\
\text { with traumatic brain injury, } 10 \text { treated with placebo and } 10 \\
\text { treated with modafinila }\end{array}$} \\
\hline & & Placebo & Modafinil & $p$ Value \\
\hline \multicolumn{5}{|c|}{ General findings } \\
\hline \multicolumn{2}{|c|}{ Age, y } & $43 \pm 19$ & $37 \pm 9$ & \\
\hline \multicolumn{2}{|c|}{ Sex, \% male } & 90 & 80 & \\
\hline \multicolumn{2}{|c|}{ Glasgow Coma Scale } & $8 \pm 4$ & $7 \pm 4$ & \\
\hline \multicolumn{2}{|c|}{ Pathologic CT findings, \% } & 60 & 50 & \\
\hline \multicolumn{2}{|c|}{ Interval after trauma, y } & $2.0 \pm 1.2$ & $1.8 \pm 0.9$ & \\
\hline \multicolumn{2}{|c|}{ High dosage (modafinil group: 200 mg), \% } & 80 & 80 & \\
\hline \multicolumn{2}{|c|}{ Side effects, \% } & 10 & 20 & \\
\hline \multicolumn{5}{|c|}{ Baseline findings } \\
\hline \multicolumn{2}{|l|}{ ESS } & $8.2 \pm 3.7$ & $10.0 \pm 4.2$ & \\
\hline \multicolumn{2}{|l|}{ FSS } & $5.0 \pm 1.4$ & $4.6 \pm 0.8$ & \\
\hline \multicolumn{2}{|c|}{ Beck Depression Inventory } & $11 \pm 9$ & $9 \pm 6$ & \\
\hline \multicolumn{2}{|c|}{ Beck Anxiety Inventory } & $10 \pm 11$ & $10 \pm 9$ & \\
\hline \multicolumn{2}{|c|}{ PSG: sleep efficiency, \% } & $87 \pm 5$ & $88 \pm 11$ & \\
\hline \multicolumn{2}{|c|}{ PSG: sleep latency to NREM2, $\min$} & $20 \pm 16$ & $16 \pm 18$ & \\
\hline \multicolumn{2}{|c|}{ PSG: apnea-hypopnea index } & $4.3 \pm 3.8$ & $2.6 \pm 3.3$ & \\
\hline \multicolumn{2}{|c|}{ PSG: periodic limb movements index } & $0.8 \pm 0.9$ & $2.1 \pm 4.4$ & \\
\hline \multicolumn{2}{|c|}{ MWT: mean sleep latency, min } & $18.7 \pm 9.3$ & $15.6 \pm 11.4$ & \\
\hline \multicolumn{2}{|c|}{ Actigraphy: awake per $24 \mathrm{~h}, \%$} & $39 \pm 13$ & $41 \pm 11$ & \\
\hline \multicolumn{2}{|c|}{ Actigraphy: sleep elongation weekend, $\mathrm{h}$} & $0.5 \pm 0.7$ & $0.8 \pm 1.0$ & \\
\hline \multicolumn{2}{|c|}{ PVT: velocity, s } & $3.7 \pm 0.6$ & $3.8 \pm 0.5$ & \\
\hline \multicolumn{2}{|c|}{ PVT: no. of lapses } & $3.7 \pm 4.3$ & $2.8 \pm 1.9$ & \\
\hline \multicolumn{5}{|c|}{ Changes from baseline } \\
\hline \multicolumn{2}{|l|}{ ESS } & $0.7 \pm 1.8$ & $-2.3 \pm 2.3$ & $0.005^{b}$ \\
\hline \multicolumn{2}{|l|}{ FSS } & $0.0 \pm 0.6$ & $-0.8 \pm 1.0$ & $0.07^{b}$ \\
\hline \multicolumn{2}{|c|}{ Beck Depression Inventory } & $-0.4 \pm 3.5$ & $1.1 \pm 3.2$ & NS \\
\hline \multicolumn{2}{|c|}{ Beck Anxiety Inventory } & $0.5 \pm 5.2$ & $-0.1 \pm 1.8$ & NS \\
\hline PSG: sle & efficiency, \% & $0.8 \pm 5.7$ & $0.0 \pm 5.1$ & NS \\
\hline PSG: sle & latency to NREM2, $\min$ & $-13.6 \pm 16.4$ & $2.7 \pm 14.7$ & $0.03^{b}$ \\
\hline MWT: $\mathrm{m}$ & n sleep latency, $\min$ & $0.4 \pm 6.2$ & $8.4 \pm 9.6$ & $0.04^{b}$ \\
\hline Actigra & : awake per $24 \mathrm{~h}, \%$ & $-0.3 \pm 4.7$ & $1.9 \pm 3.8$ & NS \\
\hline PVT: ve & ity, s & $0.0 \pm 0.2$ & $-0.2 \pm 0.4$ & NS \\
\hline PVT: no & lapses & $0.8 \pm 2.9$ & $1.3 \pm 2.6$ & NS \\
\hline
\end{tabular}

Abbreviations: ESS = Epworth Sleepiness Scale; FSS = Fatigue Severity Scale; MWT = maintenance of wakefulness test; NS = not significant; PSG = polysomnography; PVT = psychomotor vigilance test.

a Mean values are given with SDs.

${ }^{b}$ Mann-Whitney U test.
EDS. Again, ESS values at baseline did not differ between the 2 groups (modafinil: $8.2 \pm 3.7$, placebo: $10.0 \pm 4.2$ ). Relevant sleep-related breathing or movement disorders were not present in either group. The respective measures such as the apneahypopnea index or the periodic limb movement index were similar in the 2 groups. Also, actigraphy measures did not differ between groups. Beck Depression and Anxiety Inventories revealed similar results in the 2 groups. In both treatment groups, the dose of study medication was doubled in 8 patients. It was not necessary in any of these patients to reduce the dose again because of side effects. None of our patients discontinued treatment.

After 6 weeks of treatment, the decrease in FSS scores was higher in the modafinil group $(-0.8 \pm$ 1.0) than in the placebo group $(0.0 \pm 0.6)$, but this finding was not significant after correction for the independent variables sex, age, TBI severity, and Beck Depression and Anxiety scale values (figure 2). Fatigue was still present in all 8 patients in the placebo group and in 4 patients in the modafinil group. When selecting only patients with fatigue (FSS $\geq 4.0)$ at baseline, decreases in FSS scores were not higher in the modafinil group.

After 6 treatment weeks and compared with the placebo group, the decrease in ESS scores was higher in the modafinil group $(-2.3 \pm 2.3$ compared with $0.7 \pm 1.8, p=0.005$; figure 2 ). This finding remained significant after correction for the same independent variables, and also when selecting only those patients with EDS (ESS $\geq 10$ ) at baseline. Under treatment, EDS was still present in 3 patients in each group.

The objective measurement of the ability to remain awake at daytime under nonstimulating conditions revealed a significant increase compared with baseline of the mean sleep latency on MWT in the treatment group (8.4 \pm 9.6 minutes) compared with the placebo group ( $0.4 \pm 6.2$ minutes) ( $p=0.04$; figure 2). Patients under placebo treatment fell asleep quicker at nocturnal polysomnography than at baseline $(-13.6 \pm 16.4$ minutes $)$ after 6 treatment weeks, which probably reflects habituation to the sleep laboratory environment. Patients treated with modafinil, however, had no change in sleep latencies compared with baseline ( $2.7 \pm 14.7$ minutes), which again indicates decreased sleep pressure compared with the placebo group ( $p=0.03$ ). Conversely, the psychomotor vigilance test did not reveal differences between the groups: both reaction times and number of lapses were similar. Actigraphy revealed an increase of time awake per 24 hours in the modafinil group by 1.9 hours, whereas time awake decreased mildly $(-0.3$ hours) in the placebo group ( $p=$

0.33). cause of chronic sleep deprivation. A flow diagram depicting the passage of participants through this study is given in figure 1 .

At baseline, 8 patients in every group were diagnosed with fatigue. FSS values at baseline did not differ between the 2 groups: mean FSS values were $5.0 \pm 1.4$ in the modafinil group and $4.6 \pm 0.8$ in the placebo group. Five patients in the placebo group and 6 in the modafinil group were diagnosed with 


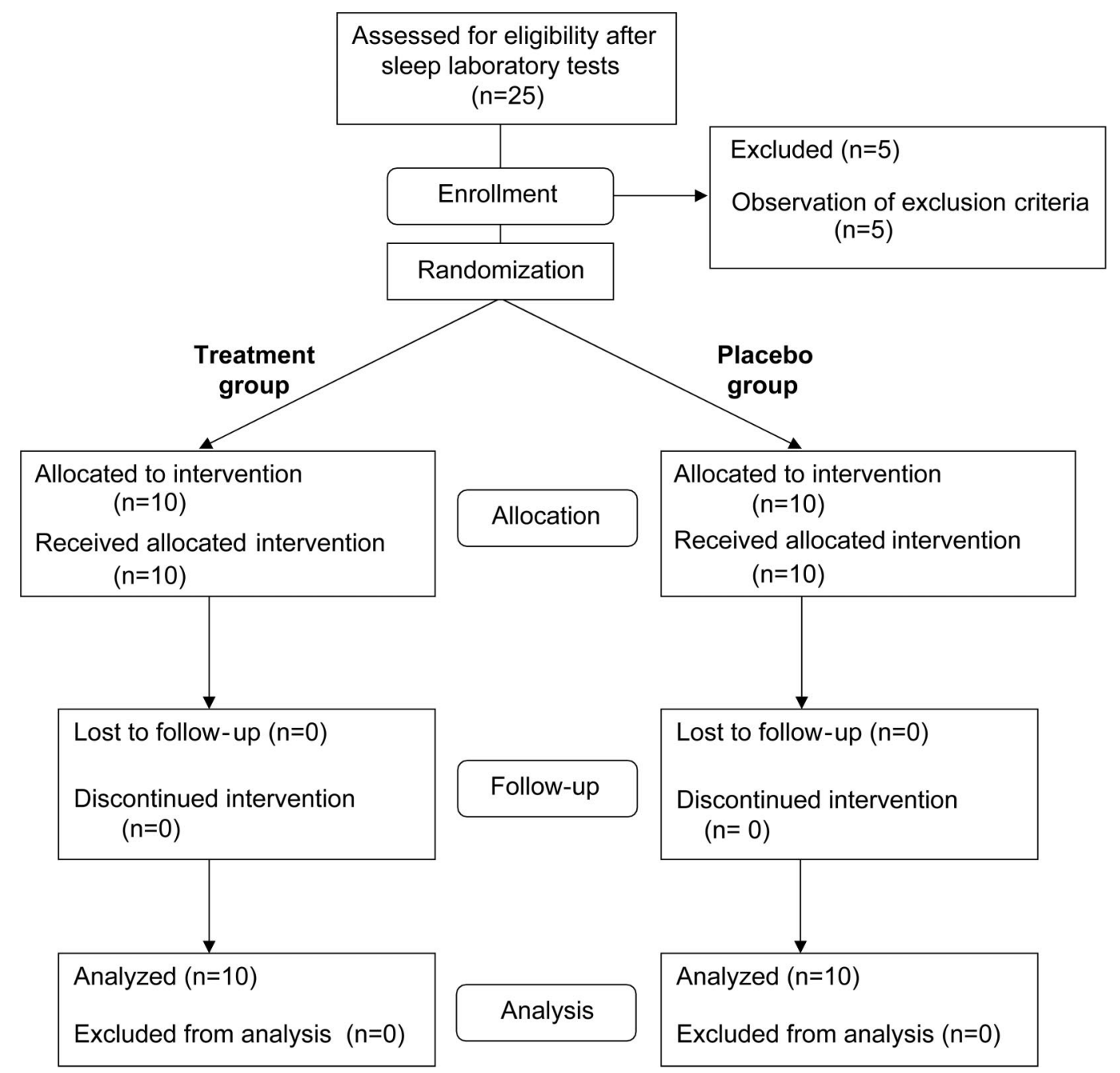

The overall subjective estimation of patients on vigilance impairment amelioration was similar in the 2 groups (table 2). These subjective self-estimations did not correlate with the FSS, the ESS, or MWT results. Depression and anxiety scale values did not change from baseline in either group. Thus, treatment-although improving objective measures of sleepiness-had no impact on quality of life. This may not be surprising, because 2.3 points on the ESS is not clinically significant, although 8.4 minutes on the MWT might be.

Side effects were similar in the 2 groups. One placebo patient reported a reduction in appetite, 1 modafinil patient reported nausea and mild and transient stomachache, and 1 modafinil patient reported mild arthralgia in the shoulders.

DISCUSSION The main outcome of this first double-blind, randomized, placebo-controlled study that included electrophysiologic sleep laboratory tests in 20 patients with TBI indicates that 100 to $200 \mathrm{mg}$ modafinil is effective and well tolerated in the treatment of posttraumatic EDS but not of fatigue. ESS values decreased by 2.3 points and mean sleep latencies on MWT increased by 8.4 minutes under treatment. Furthermore, patients with TBI who were treated with modafinil stayed awake 1.9 hours longer per day, whereas placebo-treated patients did not reveal an increase of their time awake. This potentially important difference, however, remained nonsignificant in this small trial.

Several studies observed an improvement of EDS with modafinil in a variety of disorders, including narcolepsy, obstructive sleep apnea, and chronic shift work. ${ }^{16-18}$ After TBI, EDS and fatigue are frequent complications. ${ }^{4}$ Earlier studies showed a potential benefit of modafinil for the treatment of posttraumatic vigilance impairment. ${ }^{19,20}$ Under treatment with 100 to $400 \mathrm{mg}$ modafinil (once every morning), Teitelman reported increased wakefulness and attention of posttraumatic hypersomnia in 10 patients who had closed TBI. ${ }^{19}$ Unfortunately, objective mea- 
Figure 2 Changes from baseline in ESS and FSS values, and in mean sleep latencies on MWT (in minutes)

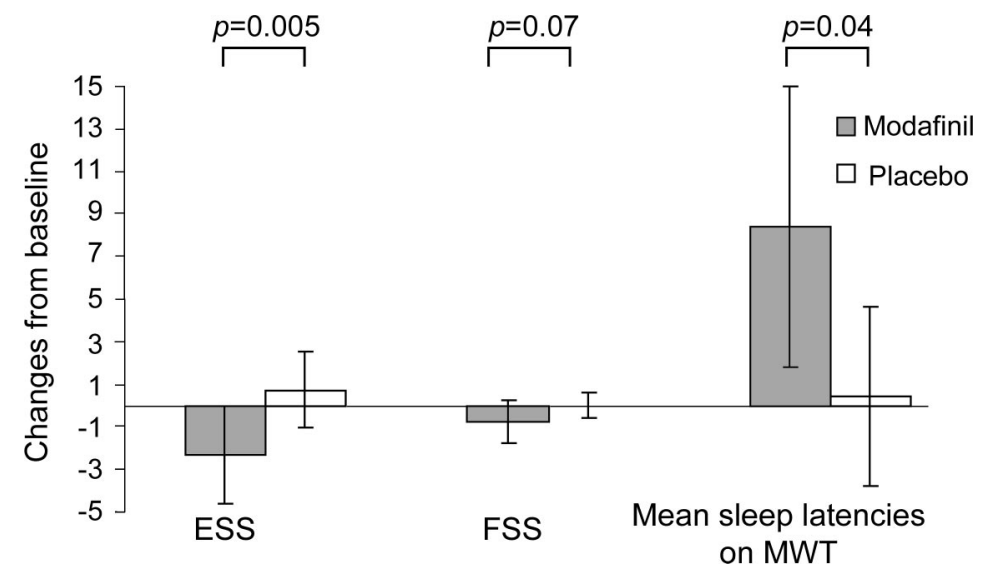

ESS $=$ Epworth Sleepiness Scale; FSS $=$ Fatigue Severity Scale; MWT = maintenance of wakefulness test. ceived placebo. This may have 2 reasons: First, based on findings in our earlier studies and in accordance with other authors, we believe that many patients with TBI tend to underestimate their sleep problems. ${ }^{4,21}$ In our first study on posttraumatic sleepwake disturbances, we found very low mean sleep latencies on multiple sleep latency tests in patients with TBI who reported that they would not be sleepy at daytime. ${ }^{4}$ Likewise, in the present study, the effect of modafinil on ESS values is smaller than in trials examining patients with narcolepsy or sleep apnea, whereas the improvement on the MWT is similar to the findings in these disorders. ${ }^{16,17}$ Therefore, the question whether patients with TBI develop a specific type of anosognosia toward sleep-wake disturbances remains to be elucidated in systematic studies. Second, in this pilot study, we administered only 100 to $200 \mathrm{mg}$ modafinil. We assume that higher doses might have a higher and clinically more significant impact on vigilance.

The lacking effect of modafinil on fatigue is remarkable. Recently, a similar outcome was observed in patients with Parkinson disease, with an improvement of EDS but not of fatigue with modafinil treatment. ${ }^{22}$ In fatigue related to multiple sclerosis or diencephalic stroke, however, modafinil was effective. ${ }^{23}$ In general, fatigue is an ill-defined symptom that probably is influenced by several factors, including physical exhaustibility, sleep-wake disturbances, and disorders of mood. Furthermore, it is not clear whether fatigue and EDS belong to the same clinical spectrum. Our result of effectiveness of modafinil for EDS but not fatigue indicates that the 2 symptoms are distinct entities, probably with different pathophysiologic pathways.

trial. In our trial as much as in this cited study, however, there is the possibility of a ceiling effect, where no higher dose of modafinil would further improve vigilance in our patients. However, because we only administered 100 to $200 \mathrm{mg}$, we cannot rule out that higher doses might add benefit for our patients.

In our study, the subjective estimation of treated patients regarding an improvement of vigilance during medication was not different from those who re-

\begin{tabular}{|llll|}
\hline Table 2 & $\begin{array}{l}\text { Overall subjective estimation } \\
\text { of vigilance impairment } \\
\text { (fatigue, excessive daytime } \\
\text { sleepiness) amelioration }\end{array}$ & \\
Much better & 1 & 0 & Placebo \\
Better & 1 & 3 & 0.23 \\
Somewhat better & 1 & 3 & \\
Unchanged & 7 & 3 & \\
Worse & 0 & 1 & \\
\hline
\end{tabular}

\section{AUTHOR CONTRIBUTIONS}

Statistical analyses were performed by Christian R. Baumann.

\section{DISCLOSURE}

Dr. Kaiser, Dr. Valko, Dr. Werth, Ms. Thomann, and Ms. Meier report no disclosures. Prof. Stocker has served on scientific advisory boards for KCI Europe Holding BV and Baxter International Inc.; has received funding for travel or speaker honoraria from Fresenius Medical Care and Bio-Synthesis Inc.; and receives research support from the Swiss National Foundation, SUVA Foundation, the Swiss Academy for Medical Sciences, and Swisstransplant. Prof. Bassetti serves as Chief Editor of the Swiss Archives of Neurology and Psychiatry and on the editorial boards of the Journal of Sleep Research, Sleep Medicine, Somnology, and the Belgian Neurological Journal; has served on scientific advisory boards for and received speaker honoraria from Boehringer Ingelheim, Lundbeck Inc., Novartis, Pfizer Inc, ResMed Inc., and UCB; receives research support from Lundbeck Inc., Respironics, Inc., ResMed Inc., Vifor Pharma, and UCB; and receives institutional research support from the Zurich Center for Integrative Human Physiology and the Swiss National Science Foundation. Dr. Baumann has served on scientific advisory boards for and received speaker honoraria from Boehringer Ingelheim, UCB, and GlaxoSmithKline; receives research support from UCB, Cephalon, and Novartis; and receives institutional research support from the Swiss National Foundation. 

2005;6:507-513.

\section{REFERENCES}

1. National Center for Injury Prevention and Control. Traumatic Brain Injury in the United States: Emergency Department Visits, Hospitalizations, and Deaths. Atlanta, GA: Centers for Disease Control and Prevention; 2006.

2. Zaloshnja E, Miller T, Janglois JA, Selassie AW. Prevalence of long-term disability from traumatic brain injury in the civilian population of the United States. J Head Trauma Rehabil 2008;23:394-400.

3. Murray CJL, Lopez AD. Global mortality, disability and the contribution of risk factors: Global Burden of Disease Study. Lancet 1997;349:1436-1443.

4. Baumann CR, Werth E, Stocker R, Ludwig S, Bassetti CL. Sleep-wake disturbances 6 months after traumatic brain injury: a prospective study. Brain 2007;130:1873-1883.

5. Masel BE, Scheibel RS, Kimbark T, Kuna ST. Excessive daytime sleepiness in adults with brain injuries. Arch Phys Med Rehabil 2001;82:1526-1532.

6. Rao V, Spiro J, Vaishnavi S, et al. Prevalence and types of sleep disturbances acutely after traumatic brain injury. Brain Inj 2008;22:381-386.

7. Castriotta RJ, Wilde MC, Lai JM, Atanasov S, Masel BE, Kuna ST. Prevalence and consequences of sleep disorders in traumatic brain injury. J Clin Sleep Med 2007;3:349-356.

8. Baumann CR, Bassetti CL, Valko PO, et al. Loss of hypocretin (orexin) neurons with traumatic brain injury. Ann Neurol 2009;66:555-559.

9. Bloch KE, Schoch OD, Zhang JN, Russi EW. German version of the Epworth Sleepiness Scale. Respiration 1999; 66:440-447.

10. Valko PO, Bassetti CL, Bloch KE, Held U, Baumann CR. Validation of the fatigue severity scale in a Swiss cohort. Sleep 2008;31:1601-1607.

11. Homaifar BY, Brenner LA, Gutierrez PM, et al. Sensitivity and specificity of the Beck Depression Inventory-II in persons with traumatic brain injury. Arch Phys Med Rehabil 2009;90:652-656.

12. King MA, Jaffre MO, Morrish E, Shneerson JM, Smith IE. The validation of a new actigraphy system for the mea-
13. Rechtschaffen A, Kales A. A Manual of Standardized Terminology, Techniques and Scoring System for Sleep Stages of Human Subjects. Washington, DC: US Public Health Service, US Government Printing Office; 1968.

14. Littner MR, Kushida C, Wise M, et al; Standards of Practice Committee of the American Academy of Sleep Medicine. Practice parameters for clinical use of the multiple sleep latency test and the maintenance of wakefulness test. Sleep 2005;28:113-121.

15. Dorrian J, Lamond N, Holmes AL, et al. The ability to self-monitor performance during a week of simulated night shifts. Sleep 2003;26:871-877.

16. Broughton RJ, Fleming JA, George CF, et al. Randomized, double-blind, placebo-controlled crossover trial of modafinil in the treatment of excessive daytime sleepiness in narcolepsy. Neurology 1997;49:444-451.

17. Black JE, Hirshkowitz M. Modafinil for treatment of residual excessive sleepiness in nasal continuous positive airway pressure-treated obstructive sleep apnea/hypopnea syndrome. Sleep 2005;28:464-471.

18. Czeisler CA, Walsh JK, Roth T, et al; U.S. Modafinil in Shift Work Sleep Disorder Study Group. Modafinil for excessive sleepiness associated with shift-work sleep disorder. N Engl J Med 2005;353:476-486.

19. Teitelman E. Off-label uses of modafinil. Am J Psychiatry 2001;158:1341.

20. Jha A, Weintraub A, Allshouse A, et al. A randomized trial of modafinil for the treatment of fatigue and excessive daytime sleepiness in individuals with chronic traumatic brain injury. J Head Trauma Rehabil 2008;23:52-63.

21. Chaumet G, Quera-Salva MA, Macleod A, et al. Is there a link between alertness and fatigue in patients with traumatic brain injury? Neurology 2008;71:1609-1613.

22. Tyne HL, Taylor J, Baker GA, Steiger MJ. Modafinil for Parkinson's disease fatigue. J Neurol 2010;257:452-456.

23. Brioschi A, Gramigna S, Werth E, et al. Effect of modafinil on subjective fatigue in multiple sclerosis and stroke patients. Eur Neurol 2009;62:243-249.

\section{Get the Latest Drug Recalls and Warnings. Give the Best Patient Care}

The American Academy of Neurology and the Health Care Notification Network have teamed up to offer AAN members a FREE online service that delivers timely neurology-specific FDAmandated patient safety drug alerts electronically.

Don't miss this opportunity to provide the best—and safest—possible care for your patients: www.aan.com/view/FDAalert 


\section{Modafinil ameliorates excessive daytime sleepiness after traumatic brain injury}

P.R. Kaiser, P.O. Valko, E. Werth, et al.

Neurology 2010;75;1780-1785

DOI 10.1212/WNL.0b013e3181fd62a2

This information is current as of November 15, 2010

\section{Updated Information \& Services}

\section{Supplementary Material}

References

Citations

Subspecialty Collections

Permissions \& Licensing

Reprints including high resolution figures, can be found at:

http://www.neurology.org/content/75/20/1780.full.html

Supplementary material can be found at: http://www.neurology.org/content/supp1/2011/05/17/75.20.1780. DC1.html

This article cites 21 articles, 3 of which you can access for free at: http://www.neurology.org/content/75/20/1780.full.html\#\#ref-list1

This article has been cited by 3 HighWire-hosted articles: http://www.neurology.org/content/75/20/1780.full.html\#\#otherart icles

This article, along with others on similar topics, appears in the following collection(s):

All Clinical trials

http://www.neurology.org//cgi/collection/all_clinical_trials

Brain trauma

http://www.neurology.org//cgi/collection/brain_trauma

Class I

http://www.neurology.org//cgi/collection/class_1

EEG

http://www.neurology.org//cgi/collection/eeg_

Other hypersomnias

http://www.neurology.org//cgi/collection/other_hypersomnias

Information about reproducing this article in parts (figures,tables) or in its entirety can be found online at:

http://www.neurology.org/misc/about.xhtml\#permissions

Information about ordering reprints can be found online: http://www.neurology.org/misc/addir.xhtml\#reprintsus 


\title{
Clinical Study
}

\section{Fatigue and Sleep-Disordered Breathing in Multiple Sclerosis: A Clinically Relevant Association?}

\author{
Ulf Kallweit, ${ }^{1,2}$ Christian R. Baumann, ${ }^{1}$ Michael Harzheim, ${ }^{2}$ Hildegard Hidalgo, ${ }^{2}$ \\ Dieter Pöhlau, ${ }^{2}$ Claudio L. Bassetti, ${ }^{3}$ Michael Linnebank, ${ }^{1}$ and Philipp O. Valko ${ }^{1}$ \\ ${ }^{1}$ Department of Neurology, University Hospital Zurich, Frauenklinikstraße 26, 8091 Zurich, Switzerland \\ ${ }^{2}$ Department of Neurology, Kamillus-Klinik, Hospitalstrasse 6, 53567 Asbach, Germany \\ ${ }^{3}$ Department of Neurology, University of Bern, Inselspital, Freiburgstrasse 10, 3010 Bern, Switzerland
}

Correspondence should be addressed to Ulf Kallweit; ulf.kallweit@usz.ch and Philipp O. Valko; philipp.valko@usz.ch

Received 12 June 2013; Revised 30 August 2013; Accepted 11 September 2013

Academic Editor: Francesca Bagnato

Copyright (C 2013 Ulf Kallweit et al. This is an open access article distributed under the Creative Commons Attribution License, which permits unrestricted use, distribution, and reproduction in any medium, provided the original work is properly cited.

\begin{abstract}
Background. Fatigue in patients with multiple sclerosis (MS) is highly prevalent and severely impacts quality of life. Recent studies suggested that sleep-disordered breathing (SDB) significantly contributes to fatigue in MS. Study Objective. To evaluate the importance of routine respirography in MS patients with severe fatigue and to explore the effects of treatment with continuous positive airway pressure (CPAP). Patients and Methods. We prospectively assessed the presence of severe fatigue, as defined by a score of $\geq 5.0$ on the Fatigue Severity Scale (FSS), in 258 consecutive MS patients. Ninety-seven patients (38\%) suffered from severe fatigue, whereof 69 underwent overnight respirography. Results. We diagnosed SDB in 28 patients (41\%). Male sex was the only independent associate of SDB severity $(P=0.003)$. CPAP therapy in 6 patients was associated with a significant reduction of FSS scores $(5.8 \pm 0.5$ versus $4.8 \pm 0.6, P=0.04)$, but the scores remained pathological $(\geq 4.0)$ in all patients. Conclusion. Respirography in MS patients with severe fatigue should be considered in daily medical practice, because SDB frequency is high and CPAP therapy reduces fatigue severity. However, future work is needed to understand the real impact of CPAP therapy on quality of life in this patient group.
\end{abstract}

\section{Introduction}

Although fatigue has been increasingly recognized over the past two decades as one of the most frequent and most debilitating symptoms in patients with MS, there are still no insights into its neurobiological mechanisms, and current treatment options are highly frustrating [1-4]. In clinical practice, MS patients complaining about fatigue are usually first scrutinized for additional and potentially treatable comorbidities, such as depression, pain, anemia, or sleepwake disturbances [5]. If there is no such cause of fatigue, the patient is considered to suffer from "MS-related fatigue," that is, a disease-inherent symptom related to the underlying neuroimmunological and neurodegenerative processes, and offlabel symptomatic treatment with stimulants of the central nervous system may be recommended [3].

Recently, the need to search for sleep-wake disorders in MS patients has been reemphasized, as several groups observed a significant correlation with fatigue [6-10]. Specifically, sleep-disordered breathing (SDB) has been proposed as a potential risk factor for fatigue in MS. In the last year, a cross-sectional study in $48 \mathrm{MS}$ patients suggested a predisposition for SDB [11], and two studies found that severe fatigue in MS was significantly associated with SDB and respiratoryrelated arousals $[9,12]$. Furthermore, Côté et al. conducted the first controlled, nonrandomized clinical treatment study and reported a significant improvement of fatigue following treatment of SDB and other sleep disorders [13]. Very recently, Veauthier et al. were able to identify treatment of sleep disorders in MS patients as independent predictor of fatigue reduction [14].

Taken together, there is increasing amount of data suggesting that SDB should be routinely screened for in all MS patients with severe fatigue. We therefore prospectively assessed the presence and severity of fatigue in consecutive, unselected MS patients seen in a tertiary center and suggested 
overnight respirography to MS patients with severe fatigue. Patients with SDB were offered CPAP therapy. The main purpose was to elucidate whether routine respirography can be recommended as a screening method in MS patients with severe fatigue and whether long-term CPAP therapy leads to a clinically meaningful improvement of fatigue. In addition, we aimed at examining potential predictive factors of SDB in MS patients with fatigue.

\section{Patients and Methods}

This prospective study was conducted at the Neurological Department of the Kamillus-Klinik in Asbach, Germany, from October 2010 until March 2011. The study protocol has been reviewed and approved by the local Institutional Review Boards, and all patients gave written informed consent before inclusion.

2.1. Subjects and Clinical Assessment. During the abovementioned period, we prospectively evaluated 258 consecutive patients with multiple sclerosis (MS). The diagnosis of MS was definite in each patient and was made according to standard criteria [15]. The majority of patients suffered from secondary progressive MS (87\%) (SPMS), and 13\% had a relapsing remitting (RRMS) form. We measured fatigue severity with the Fatigue Severity Scale (FSS), a selfadministered questionnaire with nine items which has been validated for various neurological disorders including MS $[16,17]$. A final score greater than 4.0 is widely accepted to indicate the presence of fatigue. For the purpose of our study, we included only patients with severe fatigue defined by a FSS score $\geq 5.0$. The rationale for including only patients with severe fatigue is given by the observation that beneficial treatment effects of CPAP therapy are usually most pronounced in patients with high levels of fatigue [18]. We identified 97 MS patients with severe fatigue. Sixty-nine of them agreed to undergo overnight respirography. We used the Epworth Sleepiness Scale (ESS) to assess sleepiness, with scores $\geq 10$ indicating excessive daytime sleepiness (EDS) [19, 20]. Information on disease duration was taken from the patients' medical history. We estimated the severity of disease disability using the Kurtzke Expanded Disability Status Scale (EDSS) [21].

2.2. Sleep Studies. Overnight (from 10 p.m. to 6 a.m.) respirography was performed using a portable device (EasyScreen 4.0, Respironixs, Germany), which measured nasal airflow, pulse rate, arterial oxygen saturation (finger pulse oximetry), and thoracic and abdominal movements. Respirography has been validated previously in patients with ischemic stroke [22]. Apnea was defined by a cessation of nasal airflow $\geq 10$ seconds and hypopnea by a reduction of nasal airflow by $\geq 50 \%$ or $\geq 30 \%$, when associated with an oxygen desaturation $\geq 4 \%$ [23]. Apnea-hypopnea index (AHI) was defined by the mean number of apneas and hypopneas per hour. SDB was diagnosed in patients with an $\mathrm{AHI} \geq 5 / \mathrm{h}$. SDB was classified as mild (AHI 5-15/h), moderate (AHI 15-30/h), and severe $(A H I \geq 30 / h)$. Apneas were differentiated in obstructive, mixed, and central apneas according to standard criteria [23]. Apneas were obstructive if accompanied with continuous respiratory effort, central if unaccompanied by evidence of respiratory effort, and mixed if a central apnea developed respiratory effort with evidence of obstruction later in the apneic interval. A diagnosis of central sleep apnea was made in patients, in whom $>50 \%$ of all respiratory events were central. In addition, calculation of the oxygen desaturation index (ODI) was performed. The magnitude of the oxygen desaturation considered for ODI was $\geq 4 \%$. The analysis was made automatically and corrected visually. Titration of CPAP was performed during overnight videopolysomnography. The optimal therapeutic pressure was determined to eliminate snoring, apneic events with arousals, and oxyhemoglobin desaturations in all body positions and sleep stages. Patients received conventional CPAP therapy (REMstar M series, Philips Respironics or SOMNOcomfort2, Weinmann). Reevaluation of FSS and ESS scores was scheduled after a 6-month period of CPAP therapy, although significant short-term relief of both fatigue and sleepiness can be expected already after 3-6 weeks of CPAP therapy [18].

2.3. Statistics. Continuous data were expressed as means and standard deviation (SD) and categorical variables as numbers and percentage. All analyses were performed with the Statistical Package for the Social Sciences (SPSS, version 17.0). For univariate analysis we used Student's $t$-test (for numerical scale variables) and $\chi^{2}$-test (for nominal scale variables). To identify predictors of SDB severity, we performed stepwise multiple linear regression analysis for multivariate analysis, with AHI as dependent variable and the following independent variables: age, sex, disease duration, disease subtype, EDSS, BMI, FSS, and ESS scores. Correlation analyses were performed using the Spearman's coefficient. Significance was accepted at $P<0.05$.

\section{Results}

3.1. Demographic and Clinical Characteristics. Sixty-nine MS patients with severe fatigue consented to overnight respirography. The majority were female (70\%); mean age was $49.8 \pm$ 9.2 years (range: $21-75$ years). BMI was $26.0 \pm 4.9$ (range: 17-40). Most patients were severely affected by the disease, as reflected by a mean EDSS of $5.8 \pm 1.4$ (range: $1.0-8.5$ ) and mean disease duration of $13.7 \pm 8.8$ years (range: $1-33$ years). The patient group that declined respirography $(n=$ 28) showed similar demographic and clinical characteristics (data not shown).

3.2. Sleep-Disordered Breathing. Overnight respirography revealed a mean AHI of $9.3 \pm 16.9 / \mathrm{h}$ and a mean ODI of $10.5 \pm 14.7 \%$. SDB was found in $28 \mathrm{MS}$ patients $(41 \%)$ and was mild in 18 patients (26\%), moderate in 3 patients $(4 \%)$, and severe in 7 patients (10\%). SDB was obstructive in all but one patient. MS patients with SDB were significantly older $(P=0.01)$ and tended to be more often male $(P=0.06)$. Likewise, AHI correlated with age (rho $=0.33, P=0.006)$ but 
TABle 1: Demographic and clinical characteristics in severely fatigued MS patients with and without sleep-disordered breathing.

\begin{tabular}{lccc}
\hline & No SDB $(\mathrm{AHI}<5 / \mathrm{h})$ & SDB $(\mathrm{AHI} \geq 5 / \mathrm{h})$ & $P$ \\
\hline$n(\%)$ & $41(59 \%)$ & $28(41 \%)$ & \\
Female sex, $n(\%)$ & $32(78 \%)$ & $16(57 \%)$ & 0.06 \\
Age $(\mathrm{y})$ & $47.4 \pm 8.3$ & $53.3 \pm 9.5$ & $\mathbf{0 . 0 1}$ \\
BMI & $25.4 \pm 5.2$ & $26.8 \pm 4.2$ & 0.27 \\
Disease duration $(\mathrm{y})$ & $13.8 \pm 8.9$ & $13.6 \pm 8.8$ & 0.92 \\
Disease subtype & & & 0.73 \\
$\quad$ RRMS & 3 & 6 & \\
$\quad$ SPMS & 25 & 35 & \\
EDSS & $5.9 \pm 1.5$ & $5.8 \pm 1.3$ & 0.79 \\
FSS & $5.7 \pm 0.7$ & $5.5 \pm 0.9$ & 0.36 \\
ESS & $9.4 \pm 4.7$ & $9.7 \pm 3.8$ & 0.79 \\
EDS (ESS $\geq 10)$ & $18(44 \%)$ & $17(61 \%)$ & 0.13 \\
\hline
\end{tabular}

BMI: body mass index $\left(\mathrm{kg} / \mathrm{m}^{2}\right)$, EDS: excessive daytime sleepiness, EDSS: Expanded Disability Status Scale, ESS: Epworth Sleepiness Scale, FSS: Fatigue Severity Scale, RRMS: relapsing-remitting multiple sclerosis, SPMS: secondary progressive multiple sclerosis.

not with EDSS, disease duration, or BMI. Table 1 provides the remaining demographic and clinical characteristics (Table 1).

3.3. Excessive Daytime Sleepiness. The overall prevalence of excessive daytime sleepiness in our patient group was high (51\%). The presence of excessive daytime sleepiness, however, was similar between patients with and without SDB (61\% versus $44 \%, P=0.13$ ). Even when comparing patients with moderate-severe SDB and those without SDB, we did not detect any differences in the prevalence of SDB $(70 \%$ versus $44 \%, P=0.30$ ). Similarly, there was no correlation between ESS scores and AHI $(r=-0.05, P=0.66)$.

3.4. Sex-Related Differences. The gender ratio ( $\mathrm{f}: \mathrm{m})$ was $3.6: 1$ in patients without SDB, but the male proportion significantly increased with more severe $\operatorname{SDB}(P=0.005)$ : the ratio was $2.6: 1$ in mild SDB, $0.5: 1$ in moderate SDB, and $0.4: 1$ in severe SDB. BMI was similar in both male and female patients $(25.3 \pm 3.8$ versus $26.3 \pm 5.2, P=0.12)$, but AHI was significantly higher in male patients $(18.5 \pm 24.7$ versus $5.2 \pm 9.9, P<0.001$ ). Multiple regression analysis confirmed that male sex was an independent associate of SDB severity $(P=0.003)$. Figure 1 shows the different relative distribution of SDB in male and female patients. Otherwise, the clinical characteristics were similar in both sex groups (Table 2).

3.5. Evolution of FSS and ESS under CPAP Therapy. We recommended CPAP therapy to all patients with an AHI $\geq$ $10 / \mathrm{h}(n=14)$. Nine patients agreed, but 3 of them did not tolerate the device. The remaining 6 patients ( 2 with RRMS, 4 with SPMS) revealed a CPAP daily average adherence of $\geq 5$ hours per night during the 6 -month treatment. In these patients, AHI decreased during CPAP therapy from $39.2 \pm$ $26.3 / \mathrm{h}$ to $5.2 \pm 3.6 / \mathrm{h}(P=0.02)$, and the minimal $\mathrm{SaO}_{2}$ improved from $71.5 \pm 11.7 \%$ to $88.2 \pm 1.9 \%(P=0.02)$. EDSS remained stable $(6.3 \pm 1.3$ versus $6.3 \pm 1.2)$. At followup after

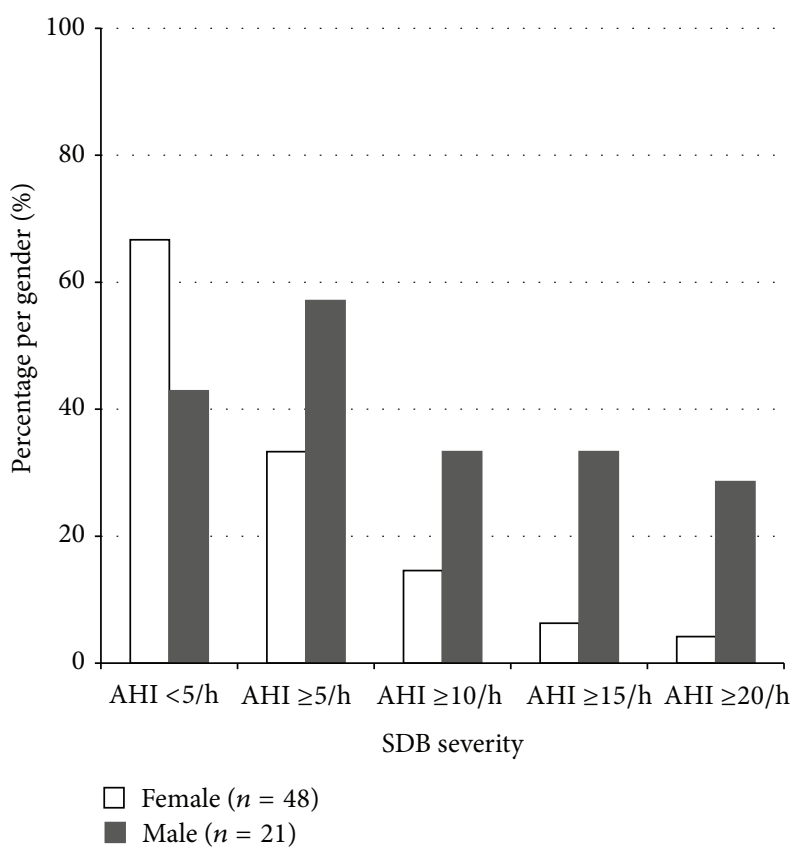

FIGURE 1: Male MS patients with severe fatigue were more frequently affected by SDB than female MS patients with severe fatigue.

TABLE 2: Gender-related differences in MS patients with severe fatigue.

\begin{tabular}{lccc}
\hline & Female & Male & $P$ \\
\hline$n(\%)$ & $48(70 \%)$ & $21(30 \%)$ & \\
Age $(y)$ & $49 \pm 9.3$ & $51 \pm 9.2$ & 0.73 \\
Disease duration $(y)$ & $14.4 \pm 9.1$ & $11.9 \pm 7.7$ & 0.16 \\
Disease subtype & & & 0.71 \\
$\quad$ RRMS & 7 & 2 & \\
$\quad$ SPMS & 41 & 19 & \\
EDSS & $5.9 \pm 1.4$ & $5.8 \pm 1.4$ & 0.78 \\
ESS & $9.7 \pm 4.5$ & $9.2 \pm 3.8$ & 0.65 \\
EDS (ESS $\geq 10)$ & $25(52 \%)$ & $10(48 \%)$ & 0.49 \\
$\mathrm{BMI}$ & $26.3 \pm 5.2$ & $25.3 \pm 3.8$ & 0.12 \\
$\mathrm{No}$ SDB $(\mathrm{AHI}<5 / \mathrm{h})$ & $32(67 \%)$ & $9(43 \%)$ & 0.06 \\
$\mathrm{AHI}$ & $5.2 \pm 9.9$ & $18.5 \pm 24.7$ & $<\mathbf{0 . 0 0 1}$ \\
$\mathrm{ODI}_{\mathrm{SaO}}$ min $(\%)$ & $7.1 \pm 7.9$ & $18.1 \pm 22.4$ & $<\mathbf{0 . 0 0 1}$ \\
& $78.9 \pm 14.6$ & $75.5 \pm 19.1$ & 0.58 \\
\hline
\end{tabular}

AHI: apnea-hypopnea index, BMI: body mass index, EDS: excessive daytime sleepiness, EDSS: Extended Disability Status Scale, ESS: Epworth Sleepiness Scale, ODI: oxygen-desaturation index, RRMS: relapsing-remitting multiple sclerosis, $\mathrm{SaO}_{2}$ min: minimal $\mathrm{O}_{2}$ saturation, $\mathrm{SDB}$ : sleep-disordered breathing, SPMS: secondary progressive multiple sclerosis.

6 months, CPAP therapy was associated with a significant decrease of FSS scores $(5.8 \pm 0.5$ versus $4.8 \pm 0.6, P=0.04)$, whereas ESS scores did not change $(9.8 \pm 3.5$ versus $9.5 \pm$ $3.0, P=0.61$ ) (Figure 2). However, despite the significant reduction of fatigue severity during CPAP therapy, FSS scores remained pathologic $(\geq 4.0)$ in all treated patients. 

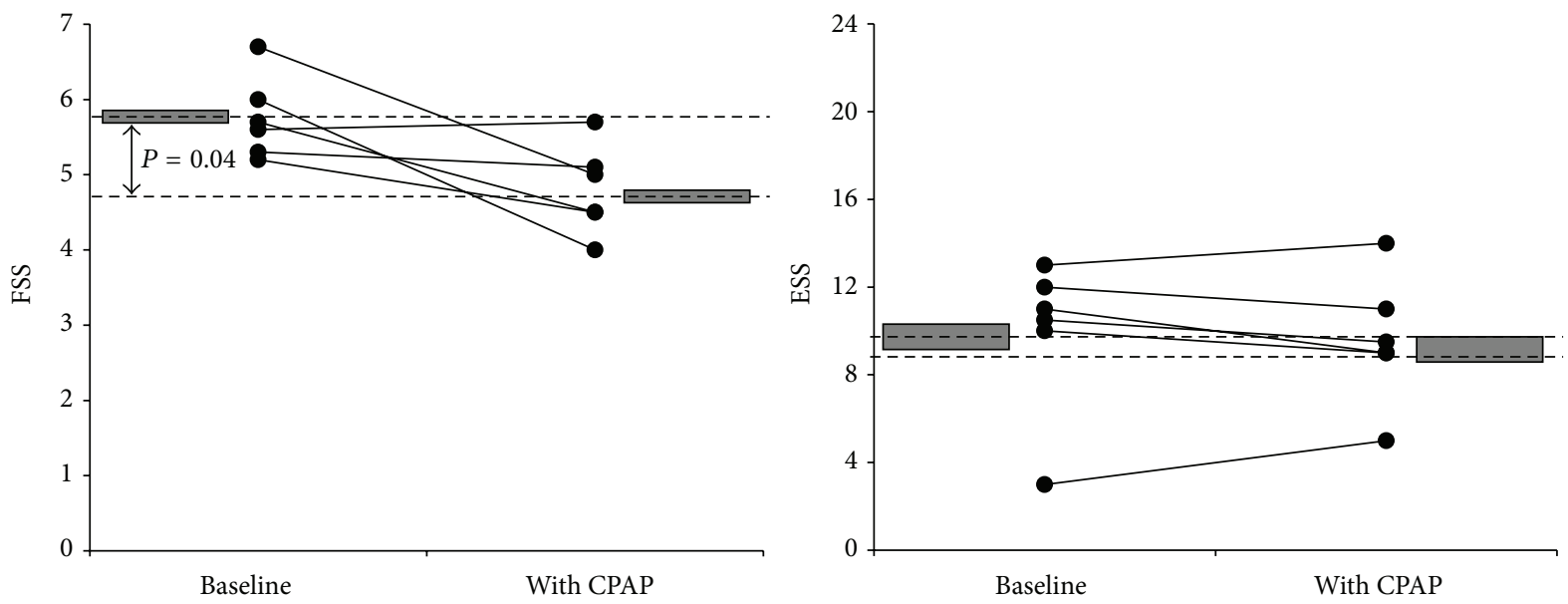

FIGURE 2: Follow-up under long-term treatment with continuous positive air pressure (CPAP) reveals significant reduction of FSS scores but not of ESS scores.

\section{Discussion}

Considering the increasing interest in SDB as a potential risk factor for fatigue in MS, the primary goal of this prospective investigation-so far the largest respirography study on SDB in MS - was to understand whether routine overnight respirography should be done in all MS patients with severe fatigue. We found a SDB frequency of $41 \%$ among MS patients with severe fatigue and demonstrated that CPAP therapy was associated with a significant improvement of fatigue severity, while sleepiness remained unchanged. Furthermore, only male sex could be identified as an sindependent predictor of SDB in MS patients with severe fatigue. Taken together, these findings indicate that routine respirography should be considered in this specific patient group, but future work is needed to confirm our results and establish the exact benefit of SDB treatment also in terms of quality of life.

Although the observed SDB frequency was rather high, our study was not designed to estimate the prevalence of SDB in MS or to compare its prevalence between MS patients with and without fatigue, as we did not perform respirography in MS patients with FSS scores $<5.0$ and did not include a control group. Moreover, comparison to other studies is hampered by disparities of included patient cohorts, methodological and technical differences, and heterogeneous definitions of SDB. In this line, two recent studies on SDB and fatigue in MS differed with regard to the applied polysomnographic scoring criteria: one group used the Rechtschaffen and Kales criteria and the other study scored according to the 1999 American Academy of Sleep Medicine Task Force [9, 12, 24]. The two methods use different placement of EEG electrodes, which may affect the scoring of respiratory arousals. Thus, several reasons may account for the large discrepancy of reported prevalence of SDB in MS patients with or without fatigue. For instance, two earlier studies did not find any cases with SDB, as defined by an $\mathrm{AHI} \geq 5 / \mathrm{h}$, among 37 and $10 \mathrm{MS}$ patients, respectively, although the majority of these patients suffered from fatigue $[25,26]$. On the other hand, Kaminska et al. performed polysomnography in 37 MS patients with severe fatigue, as defined by the same criteria as in our study, and found severe $\mathrm{SDB}(\mathrm{AHI}>30 / \mathrm{h})$ in $32 \%$, relative to $8 \%$ among 25 MS patients without severe fatigue [12]. Using another fatigue questionnaire, Veauthier et al. reported a SDB prevalence of $27 \%$ and $2.5 \%$ in $26 \mathrm{MS}$ patients with fatigue and 40 MS patients without fatigue, respectively [9]. Finally, Braley et al. reported a mean AHI of $17.0 \pm 18.8 / \mathrm{h}$ in $48 \mathrm{MS}$ patients, a much higher number than in our study [11]. Overall, these studies illustrate our uncertainty regarding prevalence and severity of SDB in MS and its relationship to fatigue.

Six MS patients with SDB and severe fatigue showed significant reduction of fatigue severity after 6 months of CPAP therapy: FSS scores decreased from $5.8 \pm 0.5$ to $4.8 \pm$ 0.6 . In other words, long-term CPAP therapy resulted in a $17 \%$ fatigue reduction. Our results are in line with the recent study of Côté et al. who treated 17 MS patients with SDB and fatigue, reporting a decrease of FSS scores from $5.0 \pm 1.7$ to $4.3 \pm 1.7$, corresponding to a $13 \%$ amelioration [13]. In addition, the reduction of mean FSS scores in both studies was $>0.6$, which has been suggested by Putzki et al. as a "clinical significant" improvement [27]. On the other hand, however, the final FSS score was still above 4.0, which is a widely accepted cut-off for fatigue [17]. Considering the high burden of MSrelated fatigue, any alleviation would certainly be desirable, even if only marginal. However, by now we do not really know to what extent the observed reductions of the FSS score represent a benefit to affected patients, especially in terms of quality of life. Worthy of mention, a multivariate analysis in the study of Côté et al. failed to detect any improvement of quality of life after CPAP therapy, as examined by the Physical and Mental Component Summary Scale of the Short Form (36) Health Survey (SF-36) [13]. The number of studies on health-related quality of life in MS patients is very large, and fatigue has been identified as an independent predictor [2830]. Thus, the lack of improvement in quality of life challenges the significance of CPAP therapy in MS-related fatigue. A possible explanation could be that the level of depression, which is an ever stronger predictor of quality of life in MS, did not improve during CPAP therapy, thereby obscuring a potential benefit [28-30]. 
Additional considerations highlight the ambiguous relationship between fatigue and SDB in MS patients. First, we currently lack any objective tool that enables us to quantify fatigue. Thus, rating of treatment outcomes regarding fatigue relies solely on subjective estimation. Second, and closely related to the first point, a possible placebo effect cannot be ruled out. As evidenced by a recent randomized, double-blind controlled trial, the placebo effect of sham CPAP therapy may be quite considerable: ESS scores decreased in the placebo group from $15.2 \pm 4.0$ to $11.9 \pm 5.9(P=0.001)$, corresponding to an effect size of 0.82 , but results of the Osler test did not change [31]. Furthermore, the placebo group showed also a significant improvement of several items of the SF36 [31]. Other groups confirmed this substantial placebo effect of CPAP therapy on sleepiness and quality of life [32]. A few groups studied fatigue response to CPAP therapy in SDB patients without underlying neurological disease, generating similar results [33-35]. While most groups uniformly reported a significant improvement of fatigue during CPAP therapy, the findings from 2 double-blind, randomized controlled trials observed significant reductions of fatigue scores also during placebo CPAP therapy $[34,35]$. Recently, Tomfohr et al. confirmed that long-term CPAP therapy leads to a benefit on fatigue beyond placebo effects [18]. In summary, these studies clearly illustrate the importance of a randomized, placebo controlled trial in order to demonstrate that the observed improvement of fatigue in MS after CPAP therapy is more than a mere placebo effect.

An intriguing finding of our study is the lack of correlation between sleepiness and SDB. Other groups made similar observations in MS patients, regarding both subjective and objective measures of sleepiness $[12,36]$. In general, sleepiness is regarded as the main daytime consequence of SDB, caused by repeated apnea-induced arousals, sleep fragmentation, and reduced slow-wave sleep, and a marked reduction of sleepiness has been demonstrated following CPAP therapy [37]. The high prevalence of sleepiness in our cohort and its persistence under CPAP therapy suggest that SDB is not the main etiology of sleepiness in MS patients with fatigue. The prevalence of most sleep-wake disturbances in MS has been shown to be increased in MS compared to the general population and may thus account for the observed high prevalence of sleepiness [7]. On the other hand, sleepiness and fatigue in MS-generally regarded as two distinct symptoms-may share a common pathophysiology. The observation that the correlation between FSS and ESS scores in MS is much stronger than in subjects with various sleep disorders is also supportive of this hypothesis $[38,39]$.

Finally, our study demonstrates that sleepiness, BMI, and disease severity are not useful predictors of SDB in MS patients with severe fatigue. Conversely, male and elder MS patients are more likely to present moderate-severe SDB. This illustrates that the presence of SDB among MS patients with severe fatigue may clinically not appear as obvious as it is in subjects with "classical" SDB.

We have to acknowledge several limitations. First of all, the number of treated patients was small, making general conclusions difficult. Second, we did not assess possible confounders, such as mood disorders, and did not measure the effects of CPAP therapy on quality of life. Third, we did not include a control group. Furthermore, the additional presence of other sleep-wake disturbances has not been assessed. The prevalence of most sleep disorders is increased in MS patients [7], and effective improvement of fatigue in MS patients may require tailored therapies of more than only one sleep disorder $[13,14]$. In particular, a recent meta-analysis indicated that restless-legs syndrome is frequent in MS, with reported prevalence of $12-58 \%$, and restless-legs syndrome appears to be associated with fatigue in MS [40]. Finally, future studies should prefer polysomnography instead of respirography, as the latter does not allow detecting apneainduced EEG arousals, and they might use additional fatigue scales such as the Modified Fatigue Impact Scale (MFIS), which has been shown to assess cognitive and psychosocial functioning more accurately than the FSS [41]. Nevertheless, we believe that our study provides an additional piece of clinical experience to the current discussion on the role of SDB in MS patients with fatigue.

In conclusion, our study confirms that SDB and fatigue are associated features in MS and that SDB treatment leads to a small yet statistically significant reduction of fatigue. However, well-designed studies are needed to substantiate the clinical relevance of this association, by proving that fatigue reduction following CPAP therapy has a meaningful impact on quality of life that goes beyond a mere placebo effect.

\section{Conflict of Interests}

All authors disclose no financial support or any other conflict of interests.

\section{Acknowledgment}

The EasyScreen was sponsored by Heinen + Löwenstein, Medizintechnik, Bad Ems, Germany.

\section{References}

[1] L. B. Krupp, L. A. Alvarez, N. G. LaRocca, and L. C. Scheinberg, "Fatigue in multiple sclerosis," Archives of Neurology, vol. 45, no. 4, pp. 435-437, 1988.

[2] J. D. Fisk, A. Pontefract, P. G. Ritvo, C. J. Archibald, and T. J. Murray, "The impact of fatigue on patients with multiple sclerosis," Canadian Journal of Neurological Sciences, vol. 21, no. 1, pp. 9-14, 1994.

[3] P. Branas, R. Jordan, A. Fry-Smith, A. Burls, and C. Hyde, "Treatments for fatigue in multiple sclerosis: a rapid and systematic review," Health Technology Assessment, vol. 4, no. 27, pp. 1-61, 2000.

[4] B. Stankoff, E. Waubant, C. Confavreux et al., "Modafinil for fatigue in MS: a randomized placebo-controlled double-blind study," Neurology, vol. 64, no. 7, pp. 1139-1143, 2005.

[5] N. Tachinana, R. S. Howard, N. P. Hursch, D. H. Miller, I. F. Moseley, and D. Fish, "Sleep problems in multiple sclerosis," European Neurology, vol. 34, no. 6, pp. 320-323, 1994. 
[6] H. P. Attarian, K. M. Brown, S. P. Duntley, J. D. Carter, and A. H. Cross, "The relationship of sleep disturbances and fatigue in multiple sclerosis," Archives of Neurology, vol. 61, no. 4, pp. 525528, 2004.

[7] S. D. Brass, P. Duquette, J. Proulx-Therrien, and S. Auerbach, "Sleep disorders in patients with multiple sclerosis," Sleep Medicine Reviews, vol. 14, no. 2, pp. 121-129, 2010.

[8] R. J. Mills and C. A. Young, "The relationship between fatigue and other clinical features of multiple sclerosis," Multiple Sclerosis, vol. 17, no. 5, pp. 604-612, 2011.

[9] C. Veauthier, H. RadbruC.H., G. Gaede et al., "Fatigue in multiple sclerosis is closely related to sleep disorders: a polysomnographic cross-sectional study," Multiple Sclerosis, vol. 17, no. 5, pp. 613-622, 2011.

[10] M. Kaminska, R. J. Kimoff, K. Schwartzman, and D. A. Trojan, "Sleep disorders and fatigue in multiple sclerosis: evidence for association and interaction," Journal of the Neurological Sciences, vol. 302, no. 1-2, pp. 7-13, 2011.

[11] T. J. Braley, B. M. Segal, and R. D. Chervin, "Sleep-disordered breathing in multiple sclerosis," Neurology, vol. 79, pp. 929-936, 2012.

[12] M. Kaminska, R. J. Kimoff, A. Benedetti et al., "Obstructive sleep apnea is associated with fatigue in multiple sclerosis," Multiple Sclerosis, vol. 18, pp. 1159-1169, 2012.

[13] I. Côté, D. A. Trojan, M. Kaminska et al., "Impact of sleep disorder treatment on fatigue in multiple sclerosis," Multiple Sclerosis, vol. 19, pp. 480-489, 2013.

[14] C. Veauthier, G. Gaede, H. Radbruch, S. Gottschalk, K. D. Wernecke, and F. Paul, "Treatment of sleep disorders may improve fatigue in multiple sclerosis," Clinical Neurology and Neurosurgery, vol. 115, no. 9, pp. 1826-1830.

[15] C. H. Polman, S. C. Reingold, G. Edan et al., "Diagnostic criteria for multiple sclerosis: 2005 Revisions to the "McDonald Criteria'"' Annals of Neurology, vol. 58, no. 6, pp. 840-846, 2005.

[16] L. B. Krupp, N. G. LaRocca, J. Muir-Nash, and A. D. Steinberg, "The fatigue severity scale. Application to patients with multiple sclerosis and systemic lupus erythematosus," Archives of Neurology, vol. 46, no. 10, pp. 1121-1123, 1989.

[17] P. O. Valko, C. L. Bassetti, K. E. Bloch, U. Held, and C. R. Baumann, "Validation of the fatigue severity scale in a Swiss cohort," Sleep, vol. 31, no. 11, pp. 1601-1607, 2008.

[18] L. M. Tomfohr, S. Ancoli-Israel, J. S. Loredo, and J. E. Dimsdale, "Effects of continuous positive airway pressure on fatigue and sleepiness in patients with obstructive sleep apnea: data from a randomized controlled trial," Sleep, vol. 34, no. 1, pp. 121-126, 2011.

[19] M. W. Johns, "A new method for measuring daytime sleepiness: the Epworth sleepiness scale," Sleep, vol. 14, no. 6, pp. 540-545, 1991.

[20] K. E. Bloch, O. D. Schoch, J. N. Zhang, and E. W. Russi, "German version of the Epworth Sleepiness Scale," Respiration, vol. 66, no. 5, pp. 440-447, 1999.

[21] J. F. Kurtzke, "Rating neurologic impairment in multiple sclerosis: an expanded disability status scale (EDSS)," Neurology, vol. 33, no. 11, pp. 1444-1452, 1983.

[22] C. Selic, M. M. Siccoli, D. M. Hermann, and C. L. Bassetti, "Blood pressure evolution after acute ischemic stroke in patients with and without sleep apnea," Stroke, vol. 36, no. 12, pp. 26142618, 2005.

[23] C. Iber, S. Ancoli-Israel, and A. Chesson, "Quan SF for the American Academy of Sleep Medicine," in The AASM Manual for the Scoring of Sleep and Associated Events: Rules, Terminology and Technical Specifications, American Academy of Sleep Medicine, Westchester, Ill, USA, 1st edition, 2007.

[24] M. Kaminska, R. J. Kimoff, A. Benedetti et al., "Obstructive sleep apnea is associated with fatigue in multiple," Multiple Sclerosis, vol. 19, pp. 372-373, 2013.

[25] B. W. Wunderlin, J. Kesselring, H. Ginzler, B. Walser, M. Kuhn, and W. H. Reinhart, "Fatigue in multiple sclerosis is not due to sleep apnoea," European Journal of Neurology, vol. 4, no. 1, pp. 72-78, 1997.

[26] H. Kaynak, A. Altintaş, D. Kaynak et al., "Fatigue and sleep disturbance in multiple sclerosis," European Journal of Neurology, vol. 13, no. 12, pp. 1333-1339, 2006.

[27] N. Putzki, Z. Katsarava, S. Vago, H. C. Diener, and V. Limmroth, "Prevalence and severity of multiple-sclerosis-associated fatigue in treated and untreated patients," European Neurology, vol. 59, no. 3-4, pp. 136-142, 2008.

[28] R. Bakshi, Z. A. Shaikh, R. S. Miletich et al., "Fatigue in multiple sclerosis and its relationship to depression and neurologic disability," Multiple Sclerosis, vol. 6, no. 3, pp. 181-185, 2000.

[29] V. Janardhan and R. Bakshi, "Quality of life in patients with multiple sclerosis: the impact of fatigue and depression," Journal of the Neurological Sciences, vol. 205, no. 1, pp. 51-58, 2002.

[30] A. J. Mitchell, J. Benito-León, J.-M. M. González, and J. Rivera-Navarro, "Quality of life and its assessment in multiple sclerosis: integrating physical and psychological components of wellbeing," Lancet Neurology, vol. 4, no. 9, pp. 556-566, 2005.

[31] M. M. Siccoli, J. C. T. Pepperell, M. Kohler, S. E. Craig, R. J. O. Davies, and J. R. Stradling, "Effects of continuous positive airway pressure on quality of life in patients with moderate to severe obstructive sleep apnea: data from a randomized controlled trial," Sleep, vol. 31, no. 11, pp. 1551-1558, 2008.

[32] J. M. Montserrat, M. Ferrer, L. Hernandez et al., "Effectiveness of CPAP treatment in daytime function in sleep apnea syndrome: a randomized controlled study with an optimized placebo," American Journal of Respiratory and Critical Care Medicine, vol. 164, no. 4, pp. 608-613, 2001.

[33] W. Chotinaiwattarakul, L. M. O’Brien, L. Fan, and R. D. Chervin, "Fatigue, tiredness, and lack of energy improve with treatment for OSA," Journal of Clinical Sleep Medicine, vol. 5, no. 3, pp. 222-227, 2009.

[34] A. Haensel, D. Norman, L. Natarajan, W. A. Bardwell, S. AncoliIsrael, and J. E. Dimsdale, "Effect of a 2 week CPAP treatment on mood states in patients with obstructive sleep apnea: a doubleblind trial," Sleep and Breathing, vol. 11, no. 4, pp. 239-244, 2007.

[35] B.-H. Yu, S. Ancoli-Israel, and J. E. Dimsdale, "Effect of CPAP treatment on mood states in patients with sleep apnea," Journal of Psychiatric Research, vol. 33, no. 5, pp. 427-432, 1999.

[36] J. P. Neau, J. Paquereau, and V. Auche, "Sleep disorders and multiple sclerosis: a clinical and polysomnography study," European Neurology, vol. 68, pp. 8-15, 2012.

[37] T. L. Giles, T. J. Lasserson, B. J. Smith, J. White, J. Wright, and C. J. Cates, "Continuous positive airways pressure for obstructive sleep apnoea in adults," Cochrane Database of Systematic Reviews, no. 1, Article ID CD001106, 2006.

[38] S. Merkelbach, H. Schulz, H. W. Kölmel et al., "What have fatigue and sleepiness in common?" Journal of Sleep Research, vol. 15, no. 1, pp. 105-106, 2006.

[39] J. L. Hossain, P. Ahmad, L. W. Reinish, L. Kayumov, N. K. Hossain, and C. M. Shapiro, "Subjective fatigue and subjective sleepiness: two independent consequences of sleep disorders?" Journal of Sleep Research, vol. 14, no. 3, pp. 245-253, 2005. 
[40] M. Schürks and P. Bussfeld, "Multiple sclerosis and restless legs syndrome: a systematic review and meta-analysis," European Journal of Neurology, vol. 20, pp. 605-615, 2013.

[41] N. Téllez, J. Río, M. Tintoré, C. Nos, I. Galán, and X. Montalban, "Does the modified fatigue impact scale offer a more comprehensive assessment of fatigue in MS?" Multiple Sclerosis, vol. 11, no. 2, pp. 198-202, 2005. 